\title{
EVALUATION OF DIFFERENT METHODS TO IDENTIFY PRECONSOLIDATION PRESSURE OF CHAMPLAIN SEA CLAY FROM CRS TESTS
}

\author{
by \\ Andries Kirstein \\ B.A.Sc., University of Ottawa, 2010 \\ A Thesis \\ presented to Ryerson University \\ in partial fulfillment of the \\ requirements for the degree of \\ Master of Applied Science \\ in the program of \\ Civil Engineering
}

Toronto, Ontario, Canada, 2017

(C) Andries Kirstein 2017 


\section{AUTHOR'S DECLARATION}

I hereby declare that I am the sole author of this thesis. This is a true copy of the thesis, including any required final revisions, as accepted by my examiners.

I authorize Ryerson University to lend this thesis to other institutions or individuals for the purpose of scholarly research.

I further authorize Ryerson University to reproduce this thesis by photocopying or by other means, in total or in part, at the request of other institutions or individuals for the purpose of scholarly research.

I understand that my thesis may be made electronically available to the public. 


\title{
EVALUATION OF DIFFERENT METHODS TO IDENTIFY PRECONSOLIDATION PRESSURE OF CHAMPLAIN SEA CLAY FROM CRS TESTS \\ Andries Kirstein
}

Master of Applied Science 2017

Department of Civil Engineering

Ryerson University

\begin{abstract}
Identifying precisely the preconsolidation pressure of any soil is one of the most challenging geotechnical problems. For sensitive soils, misjudging the preconsolidation pressure can lead to a large overestimation or underestimation of settlement due to the high compressibility after p'c. The performances of eleven graphical identification methods are evaluated on constant rate of strain (CRS) consolidation tests conducted on Champlain Sea clay. The tests include unloading/reloading cycles that are used to evaluate the accuracy of the methods based on known maximum past pressure. A number of numerical procedures are developed to aid in the use of the graphical methods for CRS test data, including locating the inflection point and the point of the maximum curvature on an $e$-logp curve. Preconsolidation pressure is calculated using straight line equations rather than interpreting it visually. These numerical methods are applied to the test data and their validities and ease of use are evaluated.
\end{abstract}




\section{ACKNOWLEDGEMENTS}

First and foremost, I would like to thank my supervisor, Dr. Jinyuan Liu. Without his support, knowledge and patience, this thesis would not have been possible.

Dr. Tareq Salloum of OPG has also been tremendously supportive, both in initiating the Waba Dam project at Ryerson University and in his regular involvement with the research.

I would like to thank my parents for their all of their support and guidance. This includes not only the last two years, but also the entire course of my lifetime.

I would also like to thank all past researchers who have contributed to the body of knowledge in this field. I have highly enjoyed studying this topic and it wouldn't have been possible without people before me laying the "groundwork".

All of my colleagues in the soils lab also deserve an enormous amount of credit. I truly appreciate that we work as a team.

Finally, I acknowledge the funding from NSERC, MITACS and OPG that made this project possible. 


\section{CONTENTS}

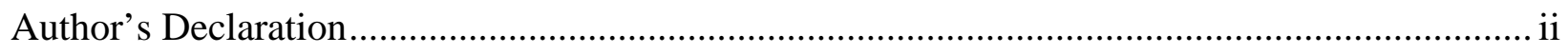

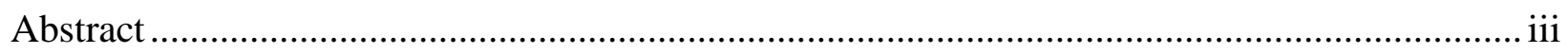

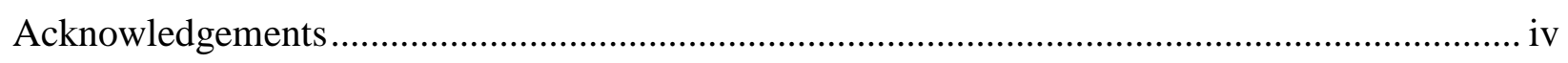

List of Tables ...................................................................................................................... viii

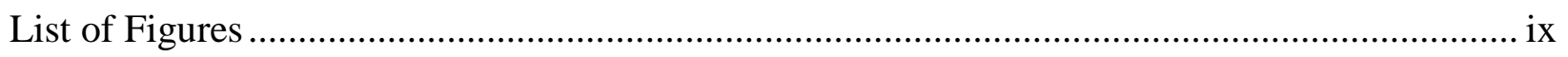

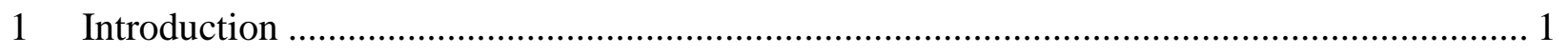

1.1 General Background........................................................................................ 1

1.2 Research Objectives ....................................................................................... 2

$1.3 \quad$ Project Background-Waba Dam.............................................................................. 2

1.4 Experimental Method........................................................................................... 5

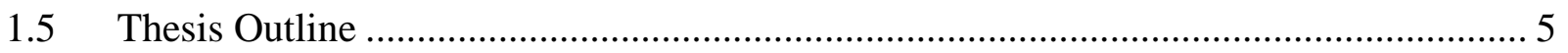

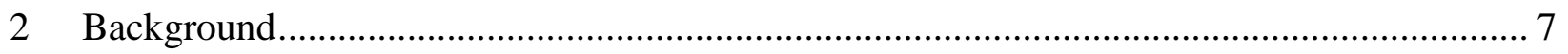

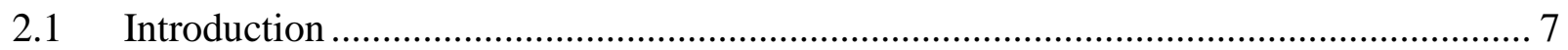

2.2 Sensitive Marine Clay …………………………..................................................... 7

2.2.1 Formation of Champlain Sea Clay ................................................................... 7

2.2.2 Effect of Pore Fluid Salinity on Sensitive Clay Properties ......................................... 8

2.2.3 Typical Properties of Sensitive Clay......................................................................... 11

2.2.4 Compression Behaviour of Sensitive Marine Clay.................................................. 12

2.3 Laval Sampling Method ............................................................................................... 14

2.4 Existing Methods to Determine Preconsolidation Pressure ............................................. 15

2.5 Previous Studies on Performances of Different Methods ............................................... 40

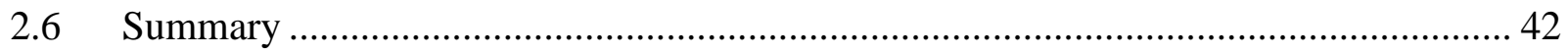

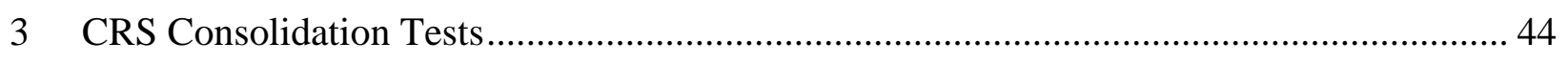

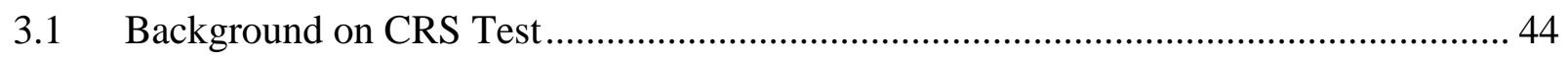

3.2 Theory of CRS Calculations ................................................................................... 44

3.3 Equations Used for Data Processing .......................................................................... 46

3.4 Experimental Procedures........................................................................................... 49

3.5 Typical Plots Available from CRS Test Data ................................................................ 52

3.6 Experimental Results on Champlain Sea Clay............................................................ 58 


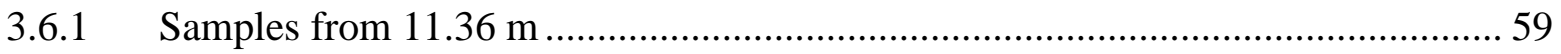

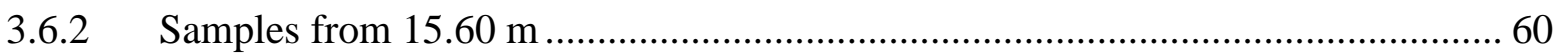

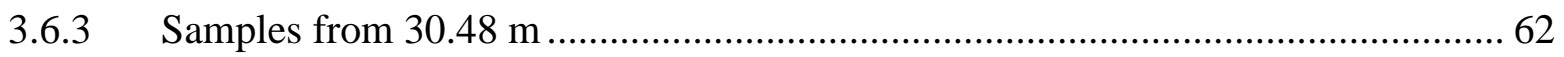

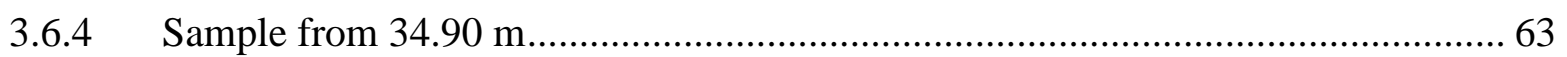

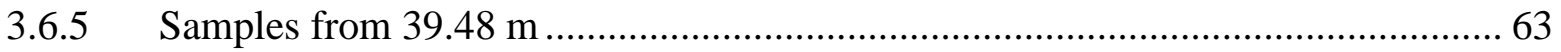

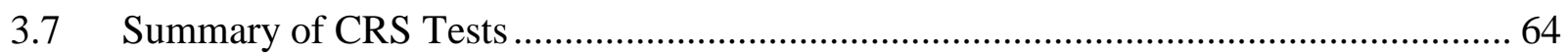

4 Numerical Techniques to Aid in Graphical Construction Methods ......................................... 65

4.1 Locating Key Points on the $e$-logp Curve .................................................................... 65

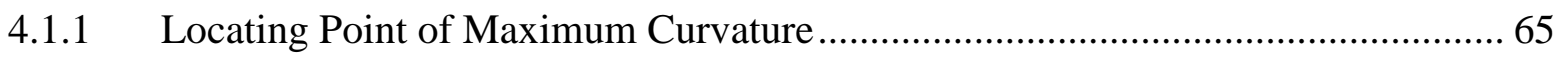

4.1.2 Locating Inflection Point .................................................................................... 70

4.2 Numerical Approaches to Graphical Methods ................................................................ 71

4.2.1 Numerical-Aided Casagrande Method ....................................................................... 71

4.2.2 Numerical-Aided Nagaraj et al. Method................................................................ 74

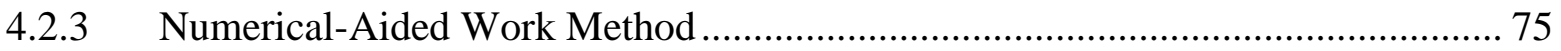

4.2.4 Numerical-Aided Wang and Frost Method............................................................... 76

4.2.5 Numerical-Aided Bilogarithmic Methods .............................................................. 77

4.2.6 Numerical-Aided Pacheco Silva Method............................................................... 78

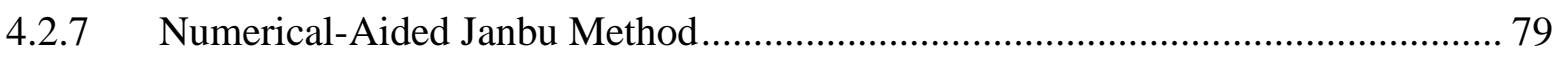

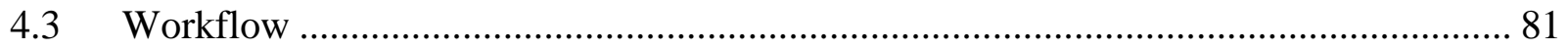

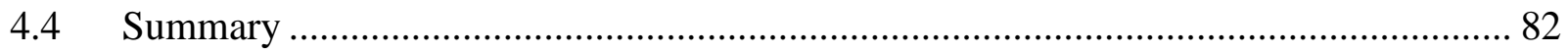

5 Evaluations of Different Methods Based on CRS Test Data................................................. 83

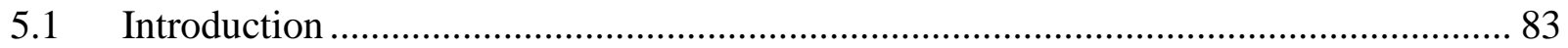

5.2 Method for Evaluation of Performance of Different Methods........................................ 83

5.2.1 Method for Evaluation using Reloading Stages..................................................... 83

5.2.2 Method for Evaluation using Initial Loading Stages .............................................. 85

5.2.3 Overview of Results from Reloading Stages ............................................................. 86

5.2.4 Overview of Results from Initial Loading Stages...................................................... 91

5.2.5 Typical CRS Test Results ......................................................................................... 96

5.3 Performance of Each Method........................................................................................ 99

5.3.1 Casagrande Method ................................................................................................ 99

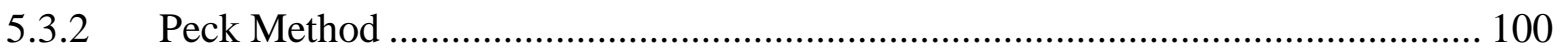




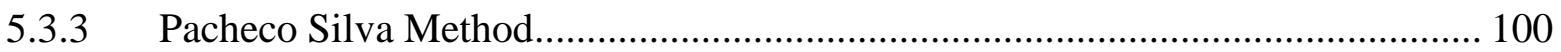

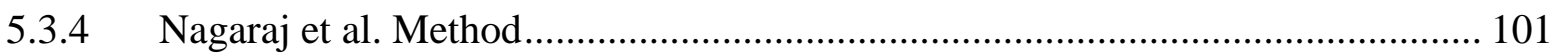

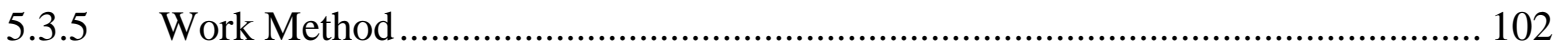

5.3.6 Wang and Frost Method........................................................................................... 103

5.3.7 Bilogarithmic Methods .................................................................................... 103

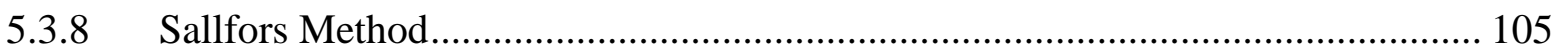

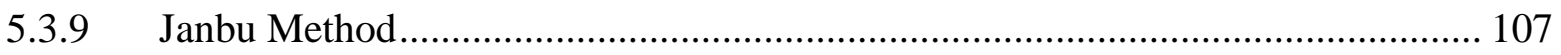

5.4 Evaluation of Numerical Methods .......................................................................... 109

5.5 Influence of Loading Strain Rate …………………............................................. 113

5.6 Influence of Sample Orientation ............................................................................. 114

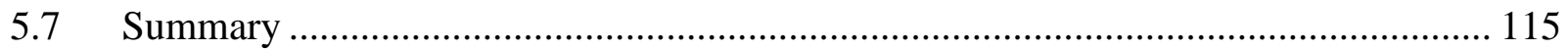

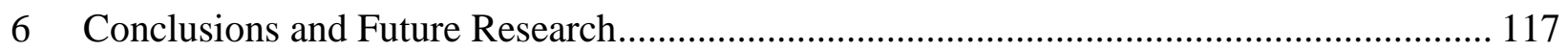

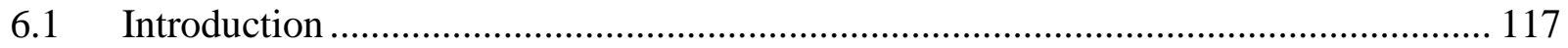

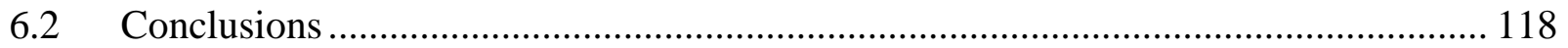

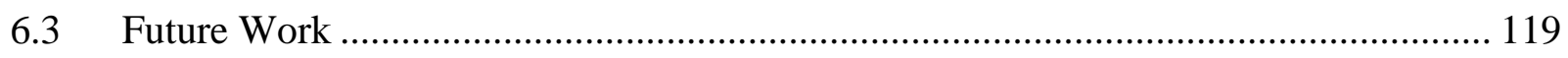

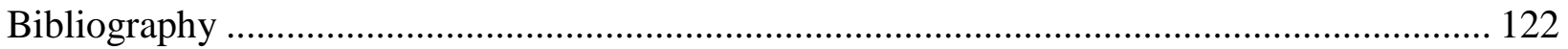




\section{LIST OF TABLES}

Table 2-1: Classification systems of soil based on sensitivity, from (Alshawmar, 2014)........... 11

Table 2-2: Typical values of the compression index, Cc (Holtz \& Kovacs, 1981) ..................... 14

Table 2-3: Conclusions of a previous study on graphical procedures to determine preconsolidation pressure (Paniagua, L'Heureux, Yang, \& Lunne, 2016) ................. 41

Table 2-4: Summary of methods included in past comparative studies ................................... 42

Table 3-1: Summary of CRS tests performed.................................................................. 58

Table 5-1: Regression equations for results of each method with known maximum past

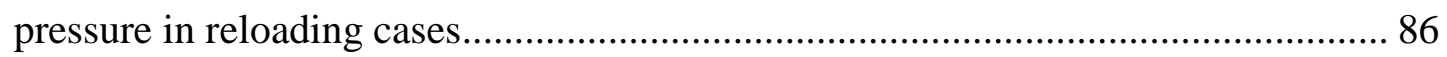

Table 5-2: Average absolute percent error of each method for the reloading tests ..................... 87

Table 5-3: Correction equations and resulting improvements to average absolute error for each method ...................................................................................................... 91

Table 5-4: Comparison of percentage differences between work method and $\ln (1+e)$ - $\ln p$ bilogarithmic method for initial loading

Table 5-5: Summary of methods used to determine maximum curvature and inflection points for all tests

Table 5-6: Influence of loading strain rate on preconsolidation pressure 113 


\section{LIST OF FIGURES}

Figure 1-1: Preconsolidation pressures obtained in the 1987 Waba Dam geotechnical investigation (Cragg, 1988) ................................................................................. 4

Figure 1-2: Results of two incremental load consolidation test with LIR=1 performed on

Waba Dam soil (Liu, Shi, \& Kirstein, 2016) …………………………………...... 5

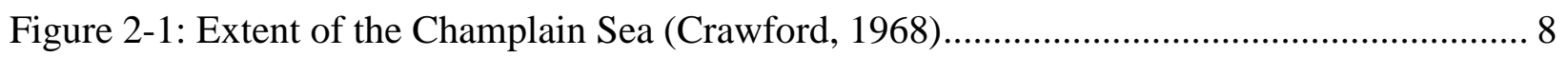

Figure 2-2: Influence of pore fluid salinity on size of electron double layer

(Rankka, et al., 2004) ........................................................................................ 9

Figure 2-3: Different types of particle connection in clay suspensions (Rankka, et al., 2004) .... 10

Figure 2-4: Effect of leaching on shear strength and compressibility of clay (Bjerrum, 1967) ... 10

Figure 2-5: Definition of the three zones of the $e$-logp curve for a sensitive clay compared with the $e$-logp curve for a remoulded sample (Nagaraj, Murthy, Vatsala, \&

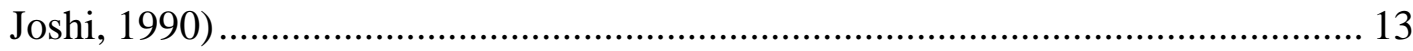

Figure 2-6: General operation of a Laval soil sampler (La Rochelle, Sarrailh, Tavenas, \&

Leroueil, 1981).............................................................................................. 15

Figure 2-7: Casagrande construction procedure for determining preconsolidation pressure

(ASTM D2435, 2011) ....................................................................................... 17

Figure 2-8: Influence of scale effect on determination of preconsolidation pressure for

Casagrande construction method and Pacheco Silva (1970) method (Clementino,

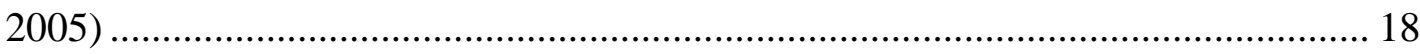

Figure 2-9: Determination of preconsolidation pressure using Peck method (Peck, Hanson,

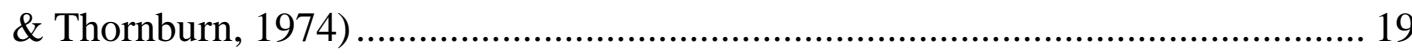

Figure 2-10: Determination of preconsolidation pressure using Pacheco Silva method

(Clementino, 2005) 20

Figure 2-11: Example of consolidation curve with two possible interpretations using

Pacheco Silva method (Boone, 2010) ..................................................................... 21

Figure 2-12: Effect of sample disturbance on e-logp curves (Nagaraj et al., 1990) ....................... 22

Figure 2-13: Determination of field preconsolidation pressure and field consolidation curve (Nagaraj, Murthy, Vatsala, \& Joshi, 1990)........................................................... 23

Figure 2-14: Determination of preconsolidation pressure using word method (Becker,

Crooks, Been, \& Jeffries, 1987)......................................................................... 24

Figure 2-15: Determination of preconsolidation pressure using dissipated strain energy method (Wang \& Frost, 2004) ........................................................................... 26

Figure 2-16: Relationship between Wang and Frost method and work method (modified from Wang \& Frost, 2004)................................................................................. 27

Figure 2-17: Consolidation data of some soils to demonstrate linearity of curves on bilogarithmic plot (Butterfield, 1979).................................................................. 28

Figure 2-18: Determination of preconsolidation pressure using the Sallfors method (Sallfors, 1975) 29 
Figure 2-19: First variation of Janbu's method for determining preconsolidation pressure (Lunne, Berre, Andersen, Sjursen, \& Mortensen, 2006) ..................................... 30

Figure 2-20: Second variation of Janbu's method for determining preconsolidation pressure

(Chung and Singh, 2015) 31

Figure 2-21: Modulus vs. stress curve for two overconsolidated, very sensitive clay samples (Janbu, 1969) 32

Figure 2-22: Typical mv vs. p’ curves for some soil types. OC = overconsolidated, NC = normally consolidated, and NCQ = normally consolidated quick clay (Janbu, 1981)

Figure 2-23: Determination of preconsolidation pressure by Karlsrud's method (Karlsrud \& Hernandez-Martinez, 2013) 33

Figure 2-24: Determination of preconsolidation pressure by Boone’s method (Boone, 2010).... 36 Figure 2-25: Use of minimum pore pressure ratio for determination of preconsolidation pressure (Seah \& Juirnarongrit, 2003) 37

Figure 2-26: Comparison of minimum pore pressure ratio with work method and other

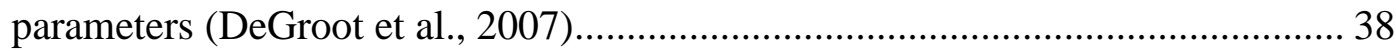

Figure 2-27: Instrumented oedometer cell with for measuring shear waves (Yoon et al., 2011). 39 Figure 2-28: Determination of preconsolidation pressure by the Yoon et al. method

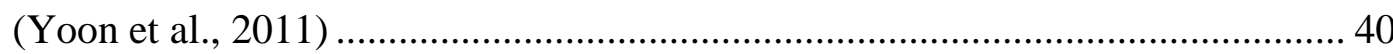

Figure 3-1: Connection of consolidation cell (Trautwein, 2001) .............................................. 49

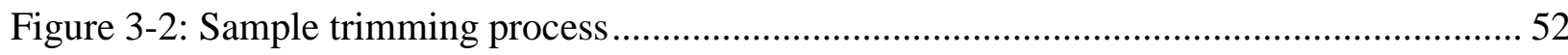

Figure 3-3: e-logp curve (AKA consolidation curve) ........................................................ 53

Figure 3-4: $e$-logk curve with data trimmed for steady state showing natural logarithmic

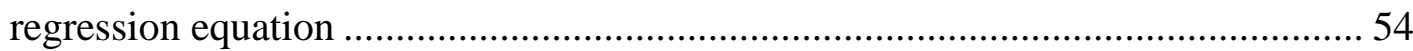

Figure 3-5: Hydraulic conductivity plotted against effective stress ........................................ 55

Figure 3-6: Coefficient of volume compressibility plotted against effective stress ................... 56

Figure 3-7: Coefficient of consolidation plotted against effective stress ................................. 57

Figure 3-8: Pore pressure ratio plotted against effective stress ............................................ 57

Figure 3-9: e-logp plot from 11.36-0.5\% test ............................................................... 59

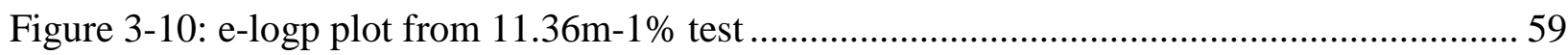

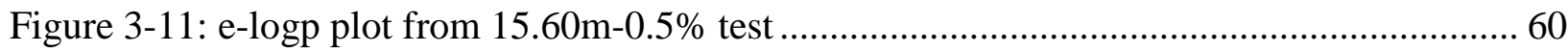

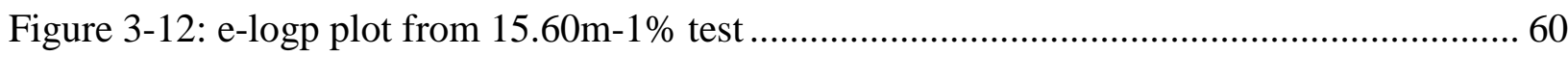

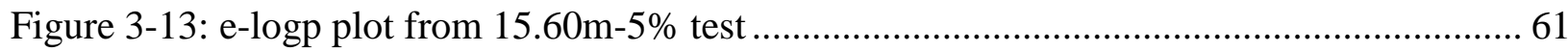

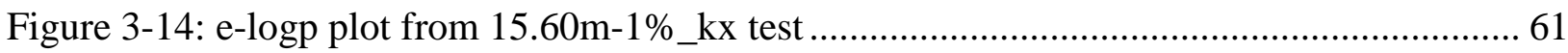

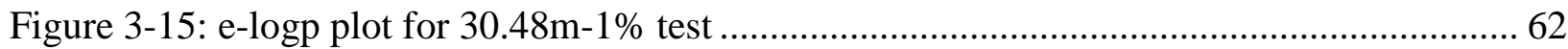

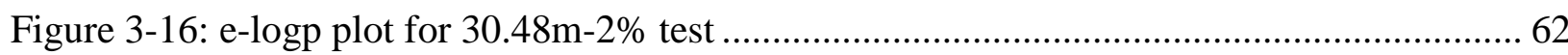

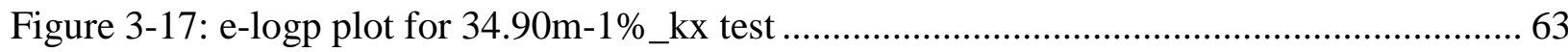

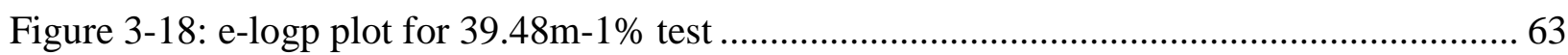

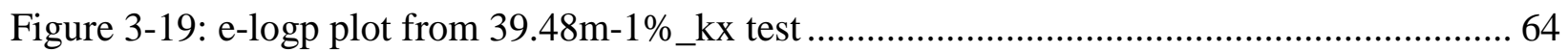


Figure 4-1: Selection of an appropriate range when calculating average slope of an $e$-logp curve.

Figure 4-2: Development of first slope of $e$-logp curve throughout a typical CRS test .............. 67

Figure 4-3: Development of second slope of $e$-logp curve throughout a CRS test .................... 68

Figure 4-4: Use of Curvature Formula to Determine Point of Maximum Curvature .................. 69

Figure 4-5: Comparison of curvature plots for different vertical scale factors.......................... 70

Figure 4-6: Determination of inflection point as point of maximum slope ............................... 71

Figure 4-7: Example of the use of formulas to perform Casagrande construction...................... 73

Figure 4-8: Adjusted scales to show location of p'c from Casagrande construction................... 73

Figure 4-9: Example of the use of formulas to perform Nagaraj et al. (1990) method ............... 75

Figure 4-10: Example of the use of formulas to perform work method .................................. 76

Figure 4-11: Comparison of Work and Dissipated Strain Energy (DSE) Methods using

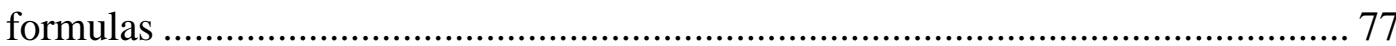

Figure 4-12: Example of the use of formulas to perform $\log (1+e)$ - $\log$ p bilogarithmic method .. 78

Figure 4-13: Example of the use of formulas to perform Pacheco Silva method....................... 79

Figure 4-14: Effect of median smoothing on constrained modulus data .................................. 79

Figure 4-15: Example of obtaining lines of best fit for Janbu method ...................................... 80

Figure 5-1: Example of obtaining known maximum past pressure .......................................... 83

Figure 5-2: Demonstration of comparing known to estimated preconsolidation pressure .......... 84

Figure 5-3: Example of plotting results obtained by a graphical method against the known

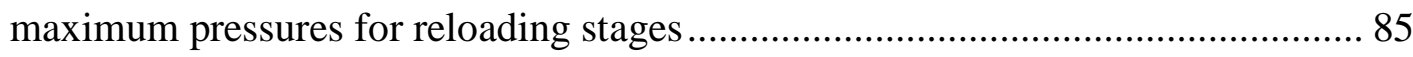

Figure 5-4: Comparison of uncorrected and corrected preconsolidation pressures in reloading tests for the Janbu method ................................................................. 88

Figure 5-5: Preconsolidation pressure vs. depth with and without correction according to

Casagrande method ..................................................................................... 95

Figure 5-6: Preconsolidation pressure vs. depth with and without correction according to

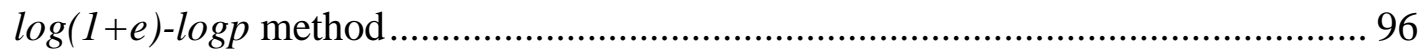

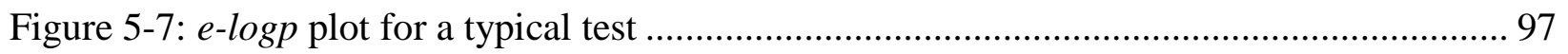

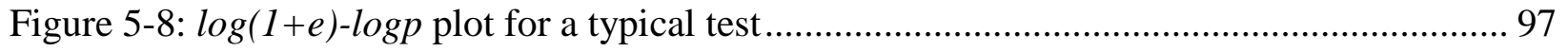

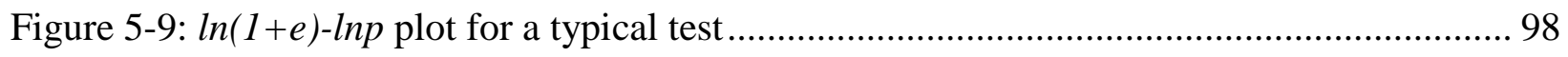

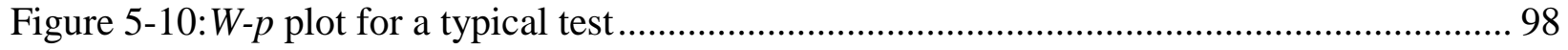

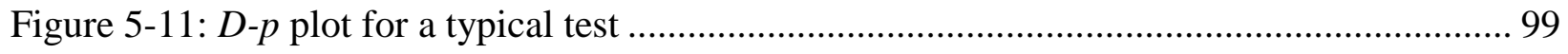

Figure 5-12: Effect of selection of point of maximum curvature on p'c obtained by method

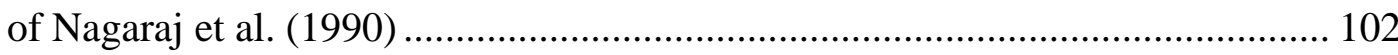

Figure 5-13: Example of a test where the consolidation curve during reloading follows a

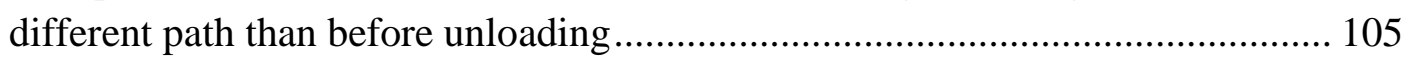

Figure 5-14: Comparison of e-logp and $\log (1+e)$-logp plots for the same test ........................ 106

Figure 5-15: Comparison of constrained modulus with slope of $e$-logp curve.......................... 107

Figure 5-16: Locations of pre-yield and post-yield regions on $D-p$ plot ................................. 108

Figure 5-17: Regions of $D$-p straight lines shown on corresponding $e$-logp plot...................... 109 
Figure 5-18: Selection of point of maximum curvature in a case where judgement is required 111 Figure 5-19: Influence of strain rate on preconsolidation pressure from CRS tests .................. 114 Figure 5-20: Influence of sample orientation on preconsolidation pressure............................. 115 


\section{INTRODUCTION}

\subsection{GENERAL BACKGROUND}

Preconsolidation pressure is often characterized as the maximum pressure that soil has experienced in its history. The preconsolidation pressure obtained in laboratory consolidation tests is, in fact, also affected by other factors such as stress release, sample disturbance, and loss of cementation bonds between particles. A more accurate term to define the change from small deformation to large deformation is "yield stress" (Burland, 1990). The term "preconsolidation pressure" will nonetheless be used in this thesis to be consistent with standard usage.

A large number of methods have been developed over the past several decades to determine the preconsolidation pressure of soil from one-dimensional consolidation test data. The majority of these methods are graphical in nature. It has been suggested that no method of determining preconsolidation pressure is guaranteed to provide a "correct" result, but that any given method should rather be evaluated based on its objectivity and consistency (Becker, Crooks, Been, \& Jeffries, 1987).

Sensitive clay shows unique behaviour in consolidation tests, the most notable of which is the "S-shape" of the $e$-logp curve (also known as the consolidation curve). Up to a certain stress level, the clay shows very little deformation with the application of additional load. After this point is surpassed, very large settlements are observed. This is thought to be due to the breaking of cementation bonds that provide the soil with its original strength. After a certain stress level, the slope of the $e$-logp curve once again decreases. The large settlements associated with sensitive clay are known to provide significant challenges for geotechnical engineers.

The influence of sample disturbance adds to the difficulty of determining the preconsolidation pressure of sensitive clay. As sample disturbance increases, the preconsolidation pressure obtained in laboratory tests decreases. Sensitive clay is highly susceptible to disturbance during extraction, and as such, specialized sampling techniques such as the Laval sampling technique have been developed for this soil type.

Because of the sudden collapse of sensitive clay during consolidation, it is difficult to accurately capture the consolidation properties of this soil in conventional oedometer tests with a loading increment ratio (LIR) of 1 . The constant rate of strain (CRS) consolidation test is widely used for sensitive clay. Reasons for this include the large number of data points available with which to better construct the consolidation curve and the rapidity of the test.

The graphical methods that have been developed for use with conventional oedometer test data can similarly be applied to data from CRS tests to determine preconsolidation pressure. These 
methods typically involve creating plots from the test data, identifying key points (e.g. point of maximum curvature) or slopes of straight portions of the curves, and then defining the preconsolidation pressure as the intersection of two straight lines that have been produced in previous steps.

Traditionally, these lines are drawn visually and these methods are considered purely graphical. However, the lines can alternatively be represented as equations using boundary conditions based on test plots, and it is possible to plot the lines mathematically. These boundary conditions are the slope of the test curve at a given point and the coordinate of that point on the curve. The preconsolidation pressure can then be determined by calculating the intersection of the equations of these lines. Due to the large number of data points collected during a CRS test, it is possible to carefully select the points used to create these straight lines. Furthermore, the point of maximum curvature can be obtained by use of a simple formula.

These simple numerical techniques are believed to reduce the subjectivity of the graphical methods, as well as eliminate certain difficulties such as reading effective stress on a logarithmic scale. These techniques are employed in this study to aid in the execution of the graphical methods, and their performance is then evaluated.

\subsection{RESEARCH OBJECTIVES}

The primary objective of this study is to evaluate a total of eleven different graphical methods of determining the preconsolidation pressure of soil from CRS consolidation test data, including one that is not found in the literature and is included for comparison with other methods. The purpose is to evaluate the performances of the different methods and find the best method to be used in a real project on sensitive Champlain Sea clay, the Wada Dam project. In addition, the conditions under which certain methods are better suited than others are evaluated. The unique considerations of CRS tests and properties of sensitive clay are taken into account in the analysis.

The secondary objective is to evaluate the validity of a number of numerical procedures that have been developed to aid in the use of the graphical procedures. The implications of the results from these numerical procedures are also investigated.

\subsection{Project Background-Waba Dam}

The soil samples used in this investigation were retrieved by a Laval sampler from below Waba Dam, an embankment dam constructed in 1976 in Arnrprior, ON.

Because of the high sensitivity of the sensitive Champlain Sea clay, a wide stabilizing berm was constructed on both the upstream and downstream sides of the dam. The profile of the foundation soil at Waba Dam can be divided into two layers, with the berm resting on top. At its deep part, the berm has a thickness of $12 \mathrm{~m}$, the top layer (labeled as upper clay) extends to $38 \mathrm{~m}$ below the 
top of the berm, and the bottom layer (labeled as lower clay) extends to $75 \mathrm{~m}$ below the top of the berm. Below the lower clay layer is a stratum of sand and till.

Due to its sensitive clay foundation, settlement to date has exceeded the maximum settlement for which it was designed. Moreover, settlement is expected to continue before consolidation is fully complete (Salloum, Cragg, Bhardwaj, \& Hassan, 2009).

A research program is currently underway at Ryerson University in collaboration with the Ontario Power Generation (OPG) to predict the long-term settlement of Waba Dam. This research program includes numerical analysis using the finite element method. The results of laboratory tests are being used as inputs for the numerical analysis. Additionally, a geotechnical profile of the foundation soil is being developed based on samples that were delivered to the laboratory.

Prior to the current investigation, two extensive geotechnical investigations have been performed on the site of Waba Dam. The first was performed in 1972 before construction. The second was performed in 1987 in order to determine the influence that the dam had had up to that time on the geotechnical properties of the underlying soil.

During the 1987 investigation, consolidation tests using incremental loading were performed to obtain values of preconsolidation pressure. Currently, there are no consolidation test records available prior to dam construction. The consolidation test results from the 1987 investigation indicate that the effective stress condition in 1987 had not yet reached the predicted final effective stress condition (Cragg, 1988). This can be seen in Figure 1-1, which presents the preconsolidation pressures obtained in the 1987 investigation, along with the estimated effective stress conditions before and after construction. As such, the current effective stress condition below Waba Dam is not known.

A number of standard laboratory tests have been performed on the Waba Dam soil samples in the Ryerson University geotechnical laboratory. Results indicate that the sensitivity of the soil ranges from 10 to 15 . The permeability of the soil is on the order of $10^{-9} \mathrm{~m} / \mathrm{s}$. The upper clay layer shows a liquidity index greater than 1 , while for the lower clay, it is less than 1 . Grain size distribution tests indicate that $97-100 \%$ of the soil is in the fine fraction (clay and silt). Salinity is seen to decrease with depth, with the highest obtained value being around $12.5 \mathrm{~g} / \mathrm{kg}$ (grams of salt per kilogram of dry soil). As expected, the bulk density increases with depth, while the water content decreases with depth, both showing an approximately linear trend with a break at the boundary between upper and lower clay. The specific gravity of the soil is within the range of 2.74-2.78 (Liu, Shi, \& Kirstein, 2016). 


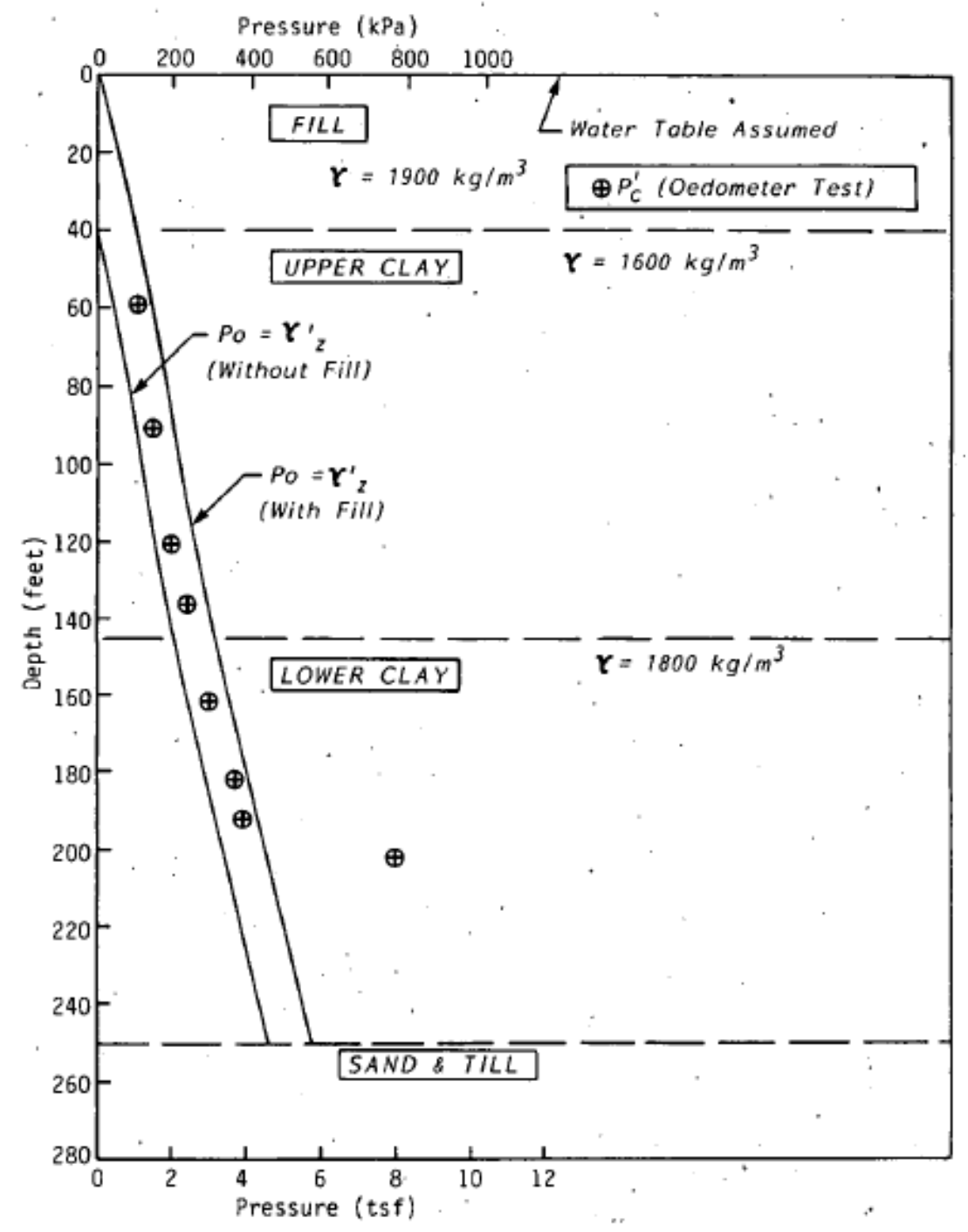

Figure 1-1: Preconsolidation pressures obtained in the 1987 Waba Dam geotechnical investigation (Cragg, 1988)

A small number of incremental oedometer tests were performed on Waba Dam soil for comparison with CRS tests. Examples are shown in Figure 1-2 for a test on Lower Clay. The preconsolidation pressure obtained using a LIR of 1 at a depth of $55.5 \mathrm{~m}$ yielded a preconsolidation pressure of $220 \mathrm{kPa}$ and a compressibility index of 0.7. These tests did not reveal any of the expected "S-shape" for sensitive clay, and the value of compressibility index is much lower than what is expected for this soil type. These results indicate that the standard incremental loading test method is not suitable for consolidation testing of Waba Dam soil. 


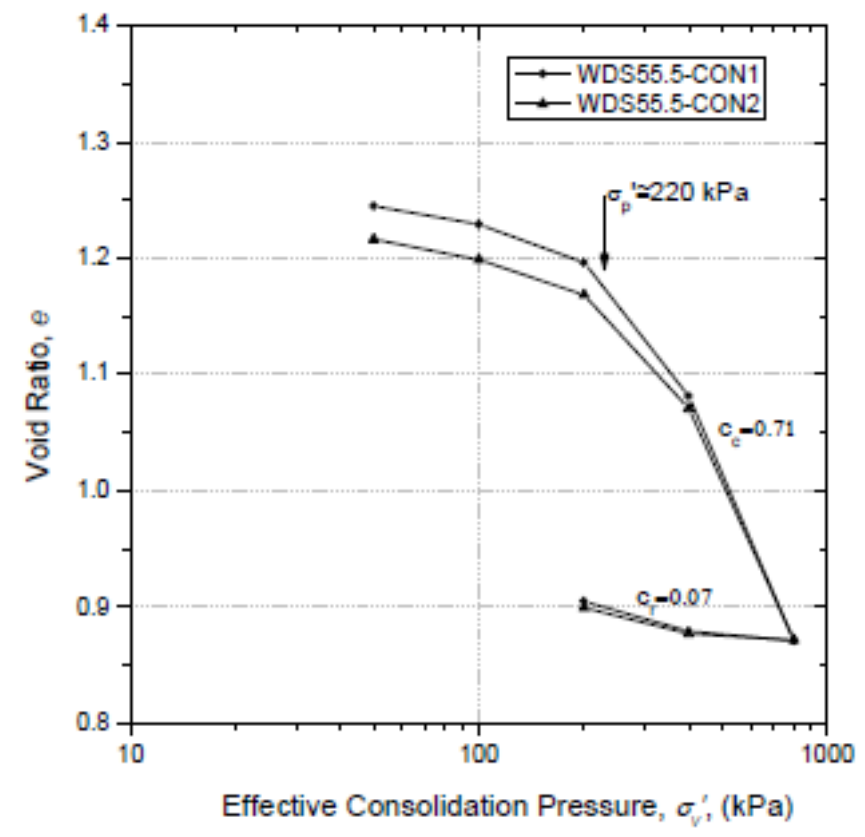

Figure 1-2: Results of two incremental load consolidation test with LIR=1 performed on Waba Dam soil (Liu, Shi, \& Kirstein, 2016)

\subsection{EXPerimental Method}

The experimental programme consisted of performing a series of CRS tests on samples of Champlain Sea clay from different depths that were retrieved by a Laval sampler from below the stabilizing berm of Waba Dam. A total of eleven tests were performed, consisting of loading, unloading, reloading and final unloading stages. In order to minimize the disturbances of the samples, the reloading stages were used to evaluate the efficacy of the different graphical methods to determine preconsolidation pressure based on the known maximum past pressure the soil had experienced before unloading. The graphical methods were also applied to the initial loading stages to provide additional data by which to compare the different methods. Since the maximum past pressure was not known in the case of the initial loading stages, the results of the different methods were compared to each other.

\subsection{Thesis OUTLINE}

In the Background section, knowledge on sensitive clay, and specifically Champlain Sea clay, will be provided. This will include the geological formation and typical properties of this soil type, as well as its behaviour in consolidation tests. Next, a brief description of the Laval sampling technique will be provided. The remainder of the background section will be devoted to describing different methods to determine the preconsolidation pressure of soil samples from 
one-dimensional consolidation tests. Ten of the fifteen methods that are described will be applied and evaluated in this investigation (including two variations of the bilogarithmic method). In addition, a bilinear method on the e-logp plot will be applied for comparison with the other bilinear methods. The remaining methods are included in the Background section to give an idea of the diversity of methods that are available to determine the preconsolidation pressure. The order in which the methods are presented is such that concepts introduced in the description of one method aid in the understanding of the next.

The section on CRS Consolidation Testing will first contain an introduction to the CRS test and some of its unique features. Next, a brief description of the theory behind the formulation equations used in CRS data processing will be presented. The formulas that were used in the data reduction for this program will then be presented. Next, the experimental procedure used for CRS testing will be outlined. Finally, a number of plots that are available from CRS tests will be shown. Some of these plots are not used in this investigation, but they are shown for the purpose of providing additional background information on the CRS test.

In the Numerical Techniques section, details will be provided on the methods used to analyze the data from the CRS tests. This will focus on the numerical methods that were developed for this investigation, including the determination of the point of maximum curvature and the inflection point of the consolidation curve. In addition, the method by which the graphical procedures are performed will be described. This involves plotting straight lines based on the known slopes and coordinates of points on the test curve and calculating the preconsolidation pressure as the intersection of these straight line equations. Finally, the median data smoothing and regression procedure used to perform the Janbu method will be described.

The section discussing Evaluations of Different Methods Based on CRS Test Data outline the rationale used in evaluating the different graphical methods. Then, an overview of the results of all the tests will be given for both the reloading and initial loading stages of the tests. After this general overview of results, the results of each method will be discussed in detail, based on the unique features and considerations of each method. In cases where interesting or unexpected results are obtained, possible explanations will be proposed. The section will conclude with a discussion and evaluation of the numerical methods that were employed in the analysis of the test results.

The Conclusions section will summarize the results that were discussed in the preceding section, as well as highlight some observations about the different methods. Ideas for future work will then be presented. The majority of future work recommendations are related to a continuation and improvement of the numerical methods that were developed in this study. 


\section{BACKGROUND}

\subsection{INTRODUCTION}

The sensitivity of a soil is defined as the ratio between its shear strength in an undisturbed state and its shear strength upon being remoulded. Most clays show a reduced shear strength after being remoulded, with the exception of some clays that have been heavily overconsolidated (Skempton \& Northey, 1952). Clays can be classified based on their sensitivities. Those with very high ratios are known as quick clays. Different systems exist to classify soils based on their degree of sensitivity, but the absolute value of sensitivity for any given soil does not change depending on what system is used.

Soils with high sensitivity have long been identified by civil engineers as a challenge (Peck, Hanson, \& Thornburn, 1974). Although clays with high sensitivity can occur anywhere depending on geological conditions, they are more commonly found in certain regions of the world. These include Scandinavia and Eastern Canada, among other regions. In Canada, sensitive clays found along the St. Lawrence River are known as Champlain Sea clays (sometimes called Leda clays). Samples of such clay are used in the current investigation.

Another notable feature of sensitive clays is that, during consolidation, they show very little deformation up to a certain level of applied stress, and then a large amount of deformation after that stress level is exceeded (Rankka, et al., 2004). This can at times cause enormous settlements of structures that are built on sensitive clay deposits. The properties of sensitive clays are heavily influenced by the salinity of their pore fluid.

In this section, the formation and behaviour of sensitive clay will be introduced. Special focus will be given to the consolidation behaviour of this soil type. A brief description of the Laval sampling method will then be provided. Finally, a number of methods to determine the preconsolidation pressure of soil from consolidation tests will be described, including a summary of past studies on the topic.

\subsection{Sensitive Marine Clay}

\subsubsection{Formation of Champlain Sea Clay}

During the most recent glacial period, glaciers with thicknesses of several hundred feet depressed the land surface of North America. When these glaciers retreated toward the end of the glacial period, marine waters flowed into what is now the St. Lawrence River valley. This formed a body of water known as the Champlain Sea (Crawford, 1968). The extent of the Champlain Sea is shown in the figure below. During this time, clay sediments that were deposited in the 
Champlain Sea settled on the sea bed in a process known as deep-water marine sedimentation (Quigley, et al., 1983). The majority of the sediment deposited in the Champlain Sea has its origin in the Canadian Shield and the primary minerals are thus quartz, feldspar, amphibole, mica, chlorite, smectite, and glacial amorphous material (Nader, 2014).

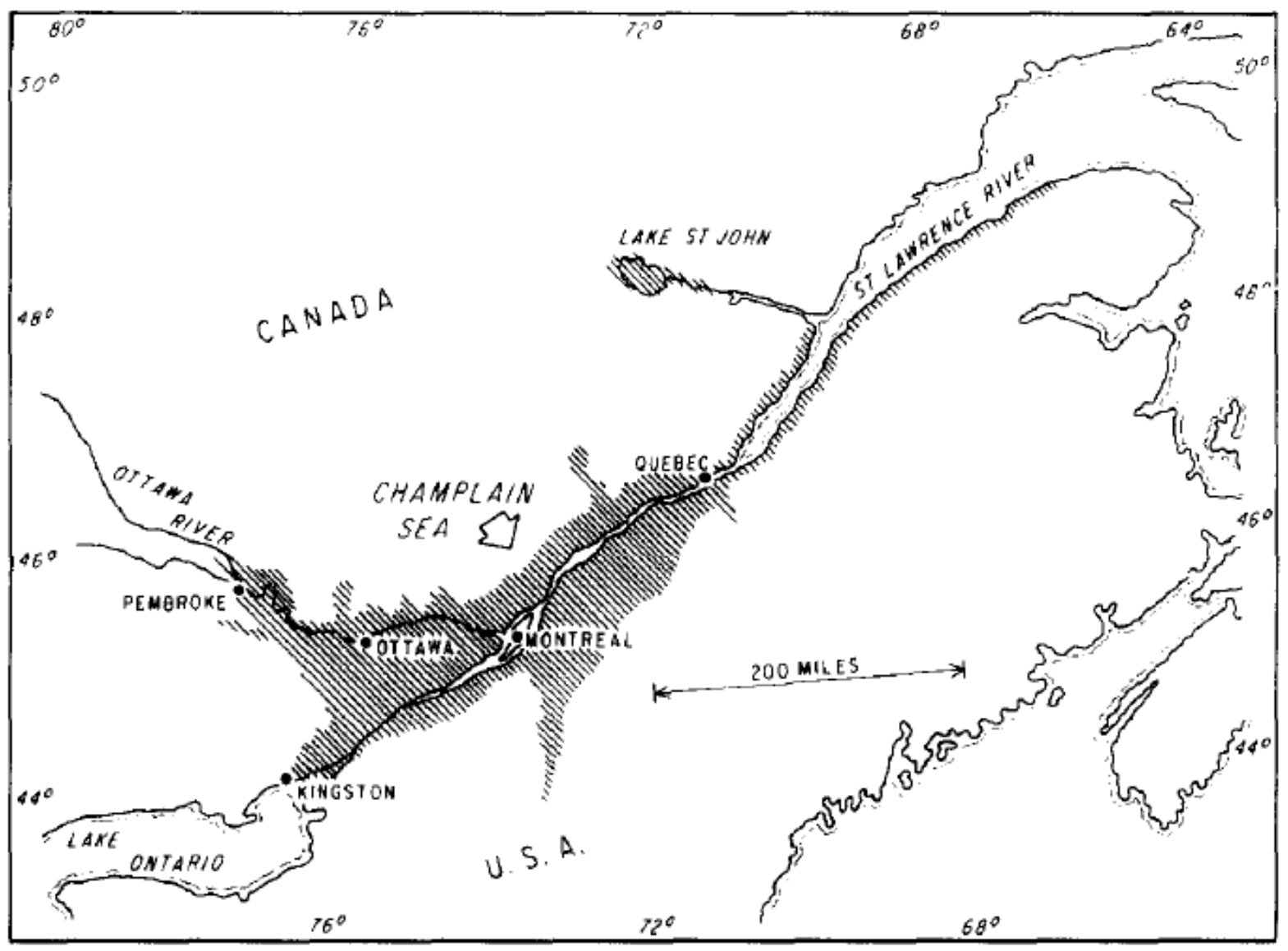

Figure 2-1: Extent of the Champlain Sea (Crawford, 1968)

Over time, the land that had been depressed by the glaciers rose by isostatic adjustment, rising by more than 700 feet. This caused the ground surface level to rise above sea level, exposing the marine sediments and causing erosion. Modern deposits of Champlain Sea clay can be as thick as $60 \mathrm{~m}$ (Quigley, et al., 1983).

\subsubsection{Effect of Pore Fluid Salinity on Sensitive Clay Properties}

Clay minerals typically have a negative charge along the particle surface, caused by the replacement of positive ions in the mineral structure by cations with lower valency. Due to this negative surface charge, positive ions present in the pore fluid are attracted to the clay particle, 
causing a high concentration of positive ions surrounding the particle. This zone of high cation concentration is known as the "electrical diffuse double layer” (Rankka, et al., 2004).

The figure below shows that, as the salinity of the pore fluid increases, the size of the electrical double layer decreases. In clays with lower pore fluid salinity, a larger electron double layer is required to neutralize the negative charge of the clay particle (Rankka, et al., 2004).

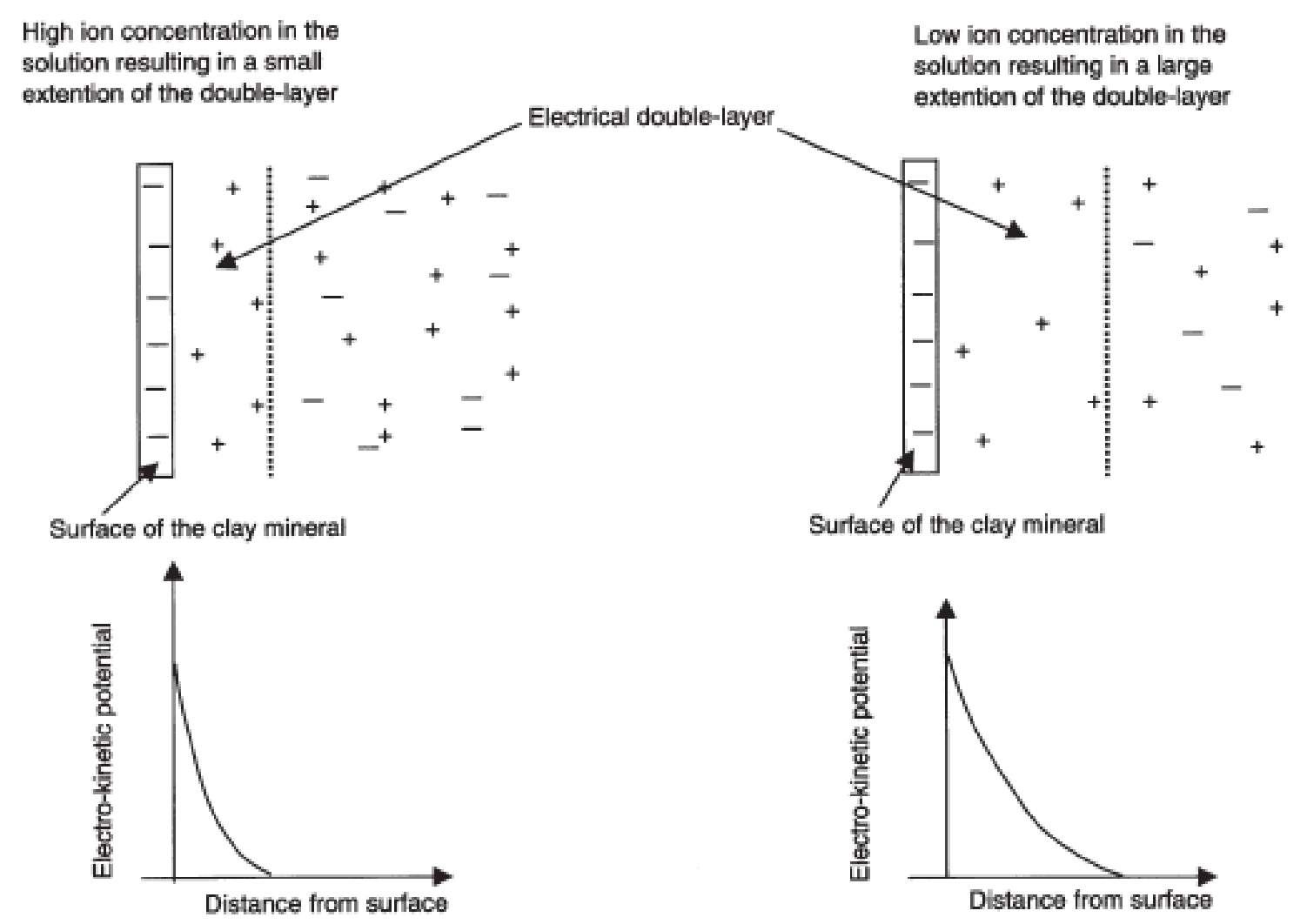

Figure 2-2: Influence of pore fluid salinity on size of electron double layer (Rankka, et al., 2004)

Due to the thin double layer around particles in high-salinity fluid, the repelling forces between clay particles are weaker and they are able to come closer together, causing flocculation. When the flocculation type is face-to-edge or edge-to-edge, the resulting clay deposit develops an open, "house of cards" particle structure with a high void ratio. This is shown in Figure 2-3. 


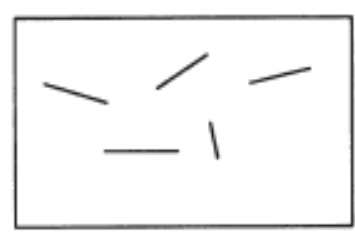

No bonds Unflocculated

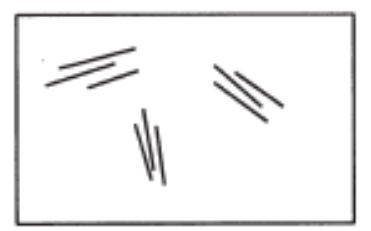

Plane to plane Unflocculated

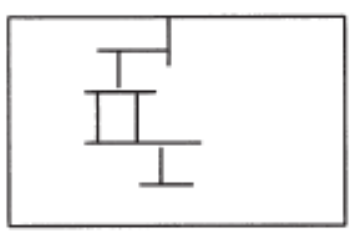

Plane to edge Flocculated

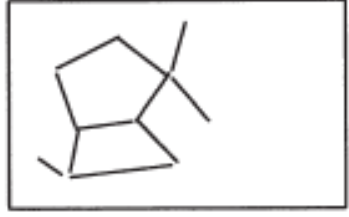

Edge to edge Flocculated

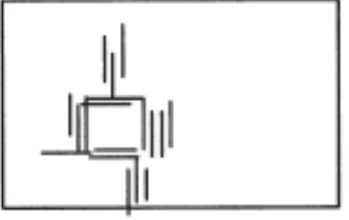

Plane to plane and plane to edge Flocculated

Figure 2-3: Different types of particle connection in clay suspensions (Rankka, et al., 2004)

The effect of loss of salinity on the compressibility of clay can be seen in Figure 2-4. The sample with leaching shown in the figure was prepared by consolidating the sample up to a load of 55 $\mathrm{kPa}$, and then holding it under the same constant load as it was leached with fresh water. This is a simulation of the geological process that occurs after the isostatic uplift of post-glacial marine clays. For the sample with leaching, it can be observed that a small increase in stress causes a much larger increase in settlement than the same stress increase for the sample without leaching (Brand \& Brenner, 1981).

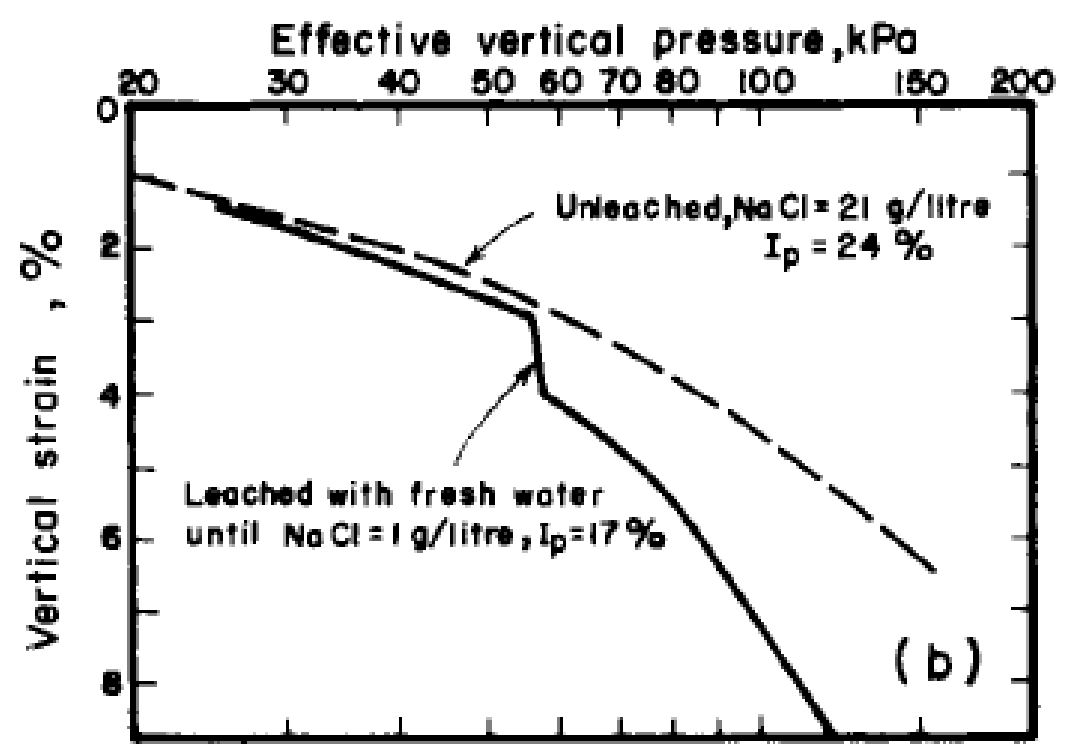

Figure 2-4: Effect of leaching on shear strength and compressibility of clay (Bjerrum, 1967) 
Loss of salinity is a major factor in the formation of sensitive clay. There are a number of mechanisms that can cause leaching (Nader, 2014). Rainfall percolating through the soil can cause salt ions to leach downward. Another mechanism by which leaching can occur is diffusion, as salt ions move to areas of lower concentration. This mechanism is much slower. It has been suggested that leaching by diffusion is less destructive of the clay skeleton since it is not accompanied by strong seepage forces, and that this might contribute to the high void ratios maintained in sensitive clays despite loss of salinity (Quigley, et al., 1983).

\subsubsection{Typical Properties of Sensitive Clay}

Different conventions have been proposed to classify soils based on sensitivity, and these are summarized in Table 2-1. Although different classification systems exist, the sensitivity of a soil is an absolute value.

Table 2-1: Classification systems of soil based on sensitivity, from (Alshawmar, 2014)

\begin{tabular}{|c|c|c|c|c|c|c|c|}
\hline \multicolumn{2}{|c|}{$\begin{array}{l}\text { Skempton and } \\
\text { Northey (1952) }\end{array}$} & \multicolumn{2}{|c|}{ Rosenqvist (1953) } & \multicolumn{2}{|c|}{ Soderblom (1969) } & \multicolumn{2}{|c|}{ Rankka et al. (2004) } \\
\hline$S_{t}$ & Classification & $S_{t}$ & Classification & $S_{t}$ & Classification & $S_{t}$ & Classification \\
\hline 1 & Insensitive & 1 & Insensitive & $<20$ & Normal & $<8$ & $\begin{array}{l}\text { Low } \\
\text { Sensitivity }\end{array}$ \\
\hline $1-2$ & $\begin{array}{c}\text { Low } \\
\text { Sensitivity }\end{array}$ & $1-2$ & $\begin{array}{l}\text { Slightly } \\
\text { Sensitive }\end{array}$ & $20-50$ & Semi-Quick & $8-30$ & $\begin{array}{l}\text { Medium } \\
\text { Sensitivity }\end{array}$ \\
\hline $2-4$ & $\begin{array}{l}\text { Medium } \\
\text { Sensitivity }\end{array}$ & $2-4$ & $\begin{array}{l}\text { Medium } \\
\text { Sensitive }\end{array}$ & $>50$ & Quick & $>30$ & $\begin{array}{c}\text { High } \\
\text { Sensitivity }\end{array}$ \\
\hline $4-8$ & Sensitive & $4-8$ & $\begin{array}{c}\text { Very } \\
\text { Sensitive }\end{array}$ & & & & \\
\hline $8-16$ & Extra Sensitive & $8-16$ & $\begin{array}{l}\text { Slightly } \\
\text { Quick }\end{array}$ & & & & \\
\hline$>16$ & Quick & $16-32$ & $\begin{array}{l}\text { Medium } \\
\text { Quick }\end{array}$ & & & & \\
\hline & & $32-64$ & Very Quick & & & & \\
\hline & & $>64$ & Extra Quick & & & & \\
\hline
\end{tabular}

In addition to high sensitivity, sensitive clays also have a number of other characteristic geotechnical properties. The first of these is a high void ratio, which results from the open, "house of cards" structure of sensitive clay. Sensitive clay also frequently has a liquidity index 
higher than 1. For normally consolidated sensitive clays, the liquidity index decreases with depth (Brand \& Brenner, 1981). Liquidity index is defined by the following equation:

$$
L I=\frac{w_{n}-P L}{L L-P L}
$$

\subsubsection{Compression Behaviour of Sensitive Marine Clay}

Determining the preconsolidation pressure of any fine grained soil is a crucial step in settlement analysis. This is often thought of as the maximum past pressure that a soil has experienced in its geological history. It should be noted that the term "vertical effective yield stress" is a more accurate term to describe the increase of deformation after a certain stress level is exceeded. This is due to the fact that the yield stress can be influenced by a number of factors other than maximum past pressure, including weathering, cementation, and mineralogical and structural factors (Boone, 2010). The term "preconsolidation pressure” will be used due to the familiarity of the term.

The task of determining the preconsolidation pressure of sensitive marine clay has historically proved to be difficult. This is in part due to the difficulty in obtaining high quality, undisturbed samples. It is widely accepted that as the disturbance of a soil sample increases, the preconsolidation pressure apparent from a consolidation test decreases. Sensitive clays are especially prone to mechanical disturbance during sampling, handling and testing (Nagaraj, Murthy, Vatsala, \& Joshi, 1990). It has been shown that, because of the sharp drop of the $e$-logp curve around the preconsolidation pressure, loading increments smaller than that used in a conventional test with increment ratio equal to 1 better define the consolidation curve. However, such tests require a much longer time to complete the test. As such, CRS tests are recommended for this soil type (Hamilton \& Crawford, 1960).

Sensitive clay has been described as having three distinct zones, labelled Zones I, II, and III, (or non-particulate, transitional, and particulate) (Nagaraj, Murthy, Vatsala, \& Joshi, 1990). In Zone I, very little deformation is seen with added stress. This is thought to be the result of resistance developed by cementation bonds between particles. The strain experienced while the soil is in this zone is typically on the order of 1\% (Nagaraj, Murthy, Vatsala, \& Joshi, 1990). There is then a sharp drop in the $e$-logp curve around the preconsolidation pressure as the soil enters Zone II. This zone represents a breakdown of the bonds holding the particles in position. After passing the transition stress, the slope of the curve once again decreases and the soil behaviour resembles that of a fully remoulded sample.

Figure 2-5 shows the shape of the e-logp curve of a sensitive clay with Zones I, II and III labelled accordingly. In the figure, $\sigma_{0}$ represents the current overburden pressure, $\sigma_{\mathrm{c}}$ represents the yield stress, and $\sigma_{\mathrm{t}}$ represents the transition stress. 


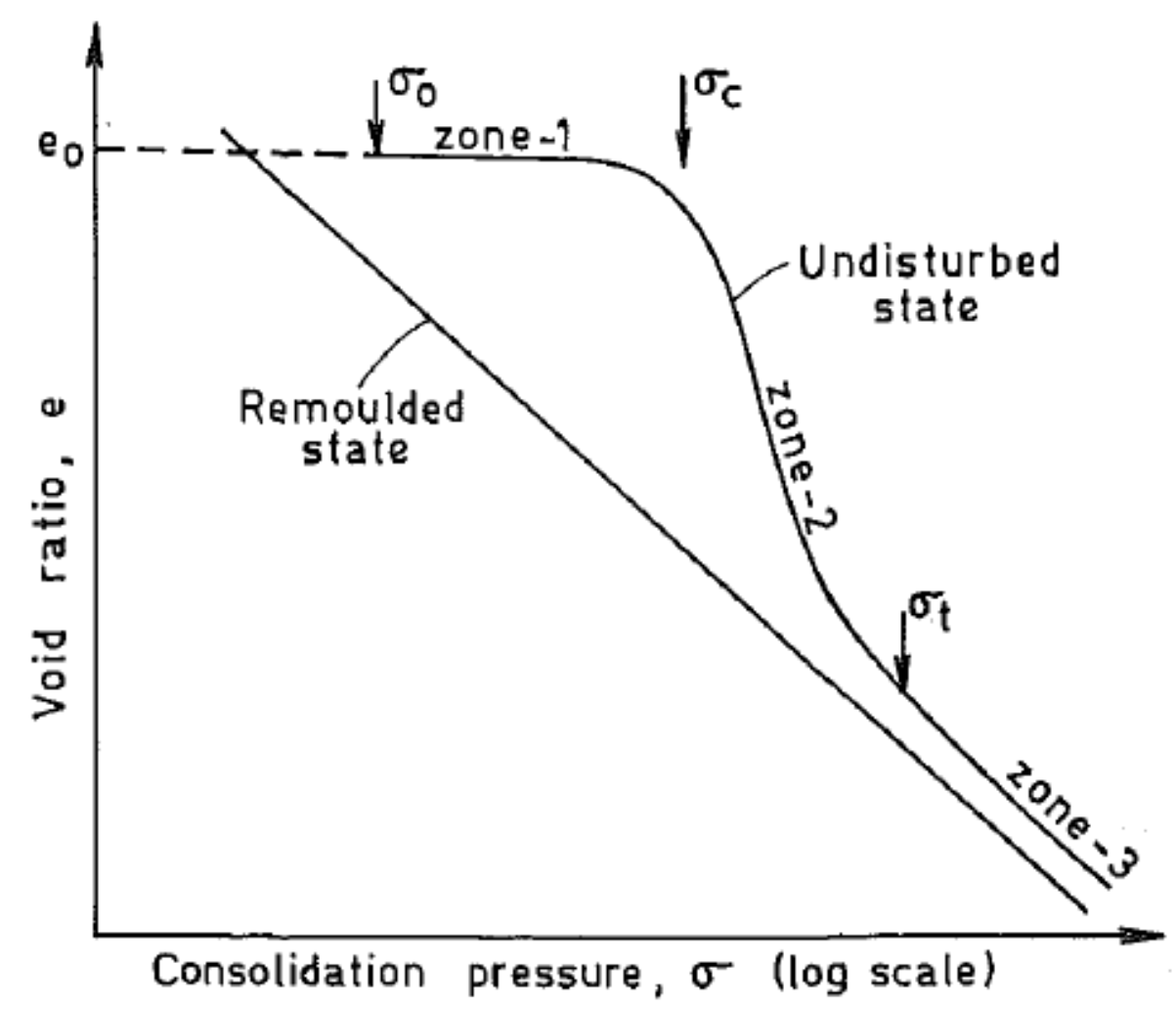

Figure 2-5: Definition of the three zones of the $e$-logp curve for a sensitive clay compared with the $e$-logp curve for a remoulded sample (Nagaraj, Murthy, Vatsala, \& Joshi, 1990)

Due to the presence of cementation bonds in Zone I, the slope of the rebound curve will be higher than the slope of the initial recompression path if the stress at unloading is higher than the preconsolidation pressure. For this reason, the Schmertmann procedure for determining the field compression curve is not valid for sensitive soils (Nagaraj, Murthy, Vatsala, \& Joshi, 1990). Since laboratory tests typically show deformation in Zone I only on the order of $1 \%$, it is supposed that the slope of this zone in the field is completely flat and that cementation bonds cause the soil to be totally rigid. This assumption is the basis of a method to predict the field preconsolidation pressure of a sensitive clay, which will be discussed in a later section that describes the Nagaraj et al. method to determine the preconsolidation pressure of a soil sample.

Some typical values for the compression index for different soil types are shown in the table below. Typical values for Canadian sensitive clays according to this table range from 1 to 4 . 
Table 2-2: Typical values of the compression index, Cc (Holtz \& Kovacs, 1981)

\begin{tabular}{ll}
\hline \multicolumn{1}{c}{ Soil } & \multicolumn{1}{c}{$C_{e}$} \\
\hline Normally consolidated medium sensitive clays & 0.2 to 0.5 \\
Chicago silty clay (CL) & 0.15 to 0.3 \\
Boston blue clay (CL) & 0.3 to 0.5 \\
Vicksburg buckshot clay (CH) & 05 to 0.6 \\
Swedish medium sensitive clays (CL-CH) & 1 to 3 \\
Canadian Leda clays (CL-CH) & 1 to 4 \\
Mexico City clay (MH) & 7 to 10 \\
Organic clays (OH) & 4 and up \\
Peats (Pt) & 10 to 15 \\
Organic silt and clayey silts (ML-MH) & 1.5 to 4.0 \\
San Francisco Bay Mud (CL) & 0.4 to 1.2 \\
San Francisco Old Bay clays (CH) & 0.7 to 0.9 \\
Bangkok clay (CH) & 0.4 \\
\hline
\end{tabular}

A method of quantifying the disturbance of clays, based on Ariake clays in Japan, has been suggested as ratio of the slope of the virgin compression curve to the slope of the remoulded compression curve (Hong \& Onitsuka, 1998). Another proposed method to quantify sample disturbance specifically for soft and sensitive clays is the ratio of the slopes of Zones I and II on the $e$-logp curve (Prasad, Triveni, Schancz, \& Nagaraj, 2007).

\subsection{LAVAl SAMpling Method}

The Laval sampling method was developed in 1981 by a team of researchers at Laval University, and is generally considered one of the most effective sampling methods in terms of keeping a sample in an undisturbed state (Clayton, Matthews, \& Simons, 1995). The intention of the Laval sampler is to achieve samples with the same high quality as block sampling but with the economic and technical feasibility of tube sampling (La Rochelle, Sarrailh, Tavenas, \& Leroueil, 1981).

The new sampler was designed to address the causes of disturbance that can be partially controlled by the design of the sampling device. Namely, these are distortion of the soil as the sampling tube is penetrated into the soil, and distortion and suction of the soil as the sampler is retrieved. The authors also highlight the release of total in situ stress as an additional source of sample disturbance, and point out that this is inevitable and cannot be addressed by an improved sampler (La Rochelle, Sarrailh, Tavenas, \& Leroueil, 1981).

The figure below shows the general operation of a Laval soil sampler. Following retrieval, samples are covered in alternating layers of wax and plastic to avoid moisture loss. 

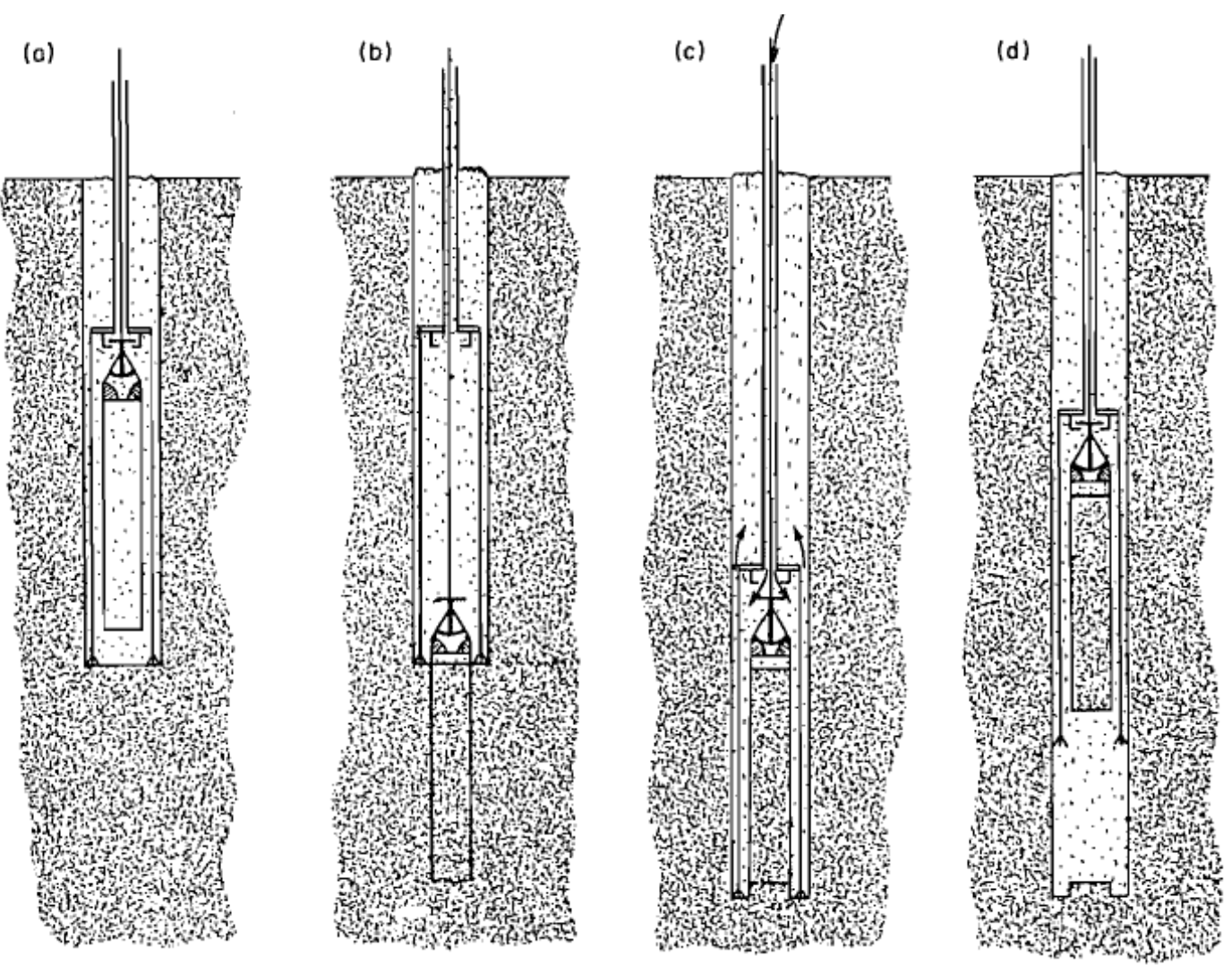

Figure 2-6: General operation of a Laval soil sampler (La Rochelle, Sarrailh, Tavenas, \& Leroueil, 1981)

\subsection{Existing Methods to Determine Preconsolidation Pressure}

Although a great number of methods have been proposed to estimate the preconsolidation pressure of a soil from 1-dimensional consolidation data, a large proportion are based on the principle that the response of a soil changes from stiff to soft as it passes the preconsolidation pressure. Examples of these methods are Casagrande (1936), Janbu (1969), Becker et al. (1987), Butterfield (1979), Oikawa (1987), Onitsuka et al. (1995), and Karlsrud (1991). Other methods are based on the principle that as the stress level surpasses the preconsolidation pressure, there is an accompanying increase in pore pressure caused by an increase in the rate of deformation. Such methods are more recently developed, and examples are those of Seah and Juirnarongrit (2003). Other methods, such as that of Yoon et al. (2011), can be classified as miscellaneous.

Another commonality between all the methods investigated is that they are based on simple graphical procedures. At the time of writing, the method of minimum pore pressure ratio (Seah \& Juirnarongrit, 2003) is the only method identified that is not graphical in nature.

The majority of the methods investigated were developed before constant rate of strain testing became common. Thus, while some of these methods are now commonly used to analyze CRS 
test data, few studies have been done to investigate their suitability to the results of a CRS test. More recently, methods have been developed to find preconsolidation pressure based exclusively on the results of CRS tests, such as that proposed by Seah and Juirnarongrit (2003).

As pointed out by Becker et al. (1987), the purpose of any method of determining preconsolidation pressure from test data is not necessarily to obtain the "correct" value, as this is essentially an impossible task. Rather, any given method should be evaluated based on its repeatability and lack of ambiguity.

Of the fifteen methods described in the following sections, only the first ten will be employed in this study (with two versions of the bilogarithmic method). A bilinear method not found in the literature will also be applied for comparison with the other bilinear methods. The remaining methods are included in the literature review to give an idea of the diversity of methods available to determine preconsolidation pressure from consolidation test data.

The order in which the methods are presented is not chronological. Rather, they will be presented in such a way that concepts introduced in the description of one method aid in the understanding of the next.

\subsubsection{Casagrande Method}

The method proposed by Casagrande (Casagrande, 1936) is very commonly in use in engineering practice. The procedure for Casagrande construction is as follows:

1. Determine the point of maximum curvature

2. At the point of maximum curvature, draw:

a. A line tangent to the test curve

b. A horizontal line

c. A line that bisects the tangent line and horizontal line

3. Extend upward a straight line approximation of the virgin consolidation curve

4. The point where the virgin consolidation extension intersects with the bisecting line is defined as the preconsolidation pressure

It is generally agreed that the selection of the point of maximum curvature is subject to the interpretation of the user. The Casagrande method is said to give good results as long as there is a well-defined break in the consolidation curve (Grozic, Lunne, \& Pande, 2003).

Although the Casagrande construction method is commonly recommended in textbooks and codes, it has been found in multiple studies to be inaccurate when applied to reloading cycles of oedometer tests with known maximum past pressures (e.g. (Umar \& Sadrekarimi, 2016)). 

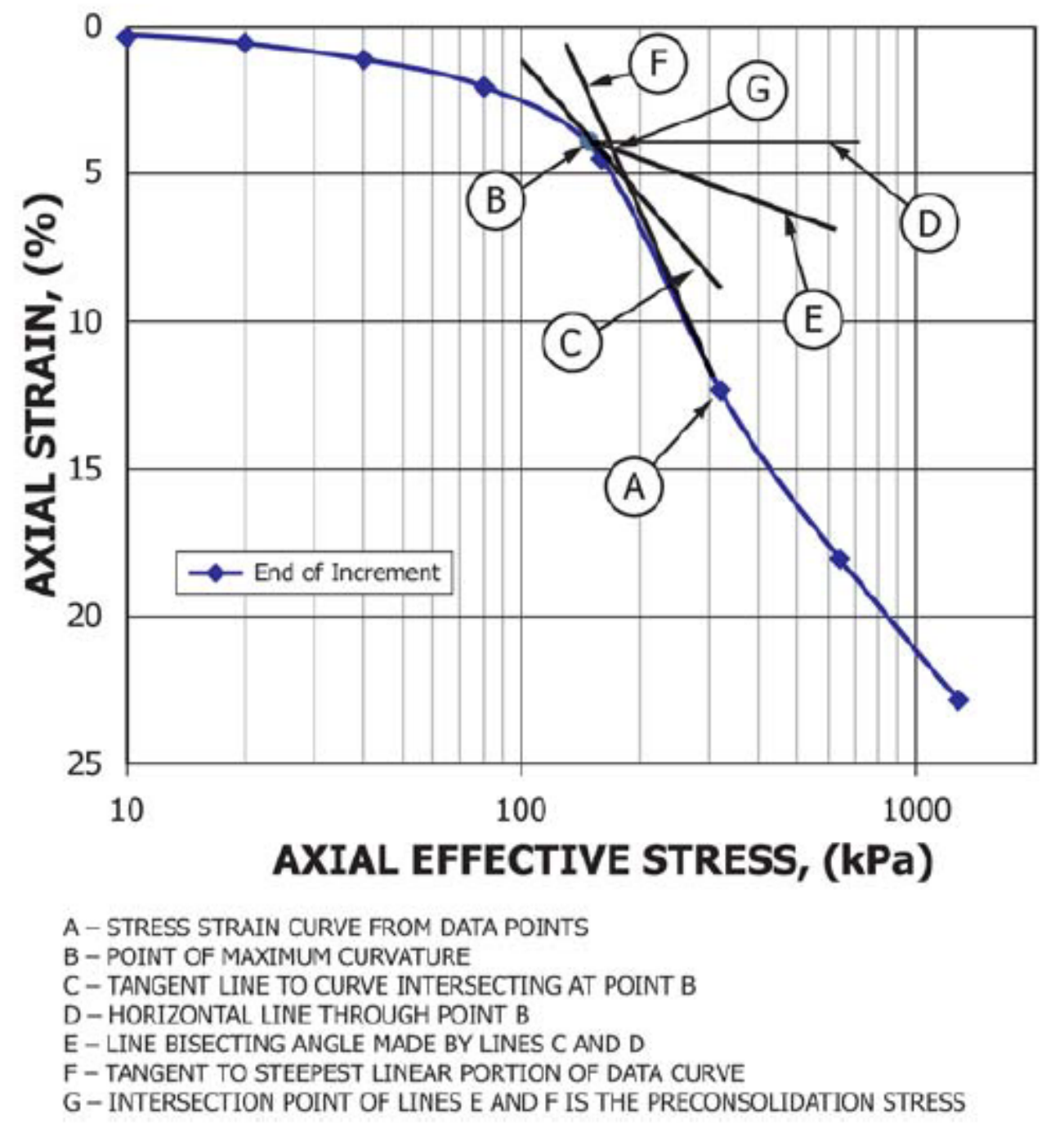

Figure 2-7: Casagrande construction procedure for determining preconsolidation pressure (ASTM D2435, 2011)

One criticism of the Casagrande construction is that the selection of the point of maximum curvature is influenced by the scale that is used for the vertical axis. As demonstrated in the figure below, an exaggerated void ratio axis will result in a lower stress value at the selected maximum curvature, and thus a lower preconsolidation pressure (Clementino, 2005). The figure compares the Casagrande construction method to the method proposed by Pacheco Silva. No mention has been found in the literature of a standardized ratio of horizontal to vertical scale factors. 


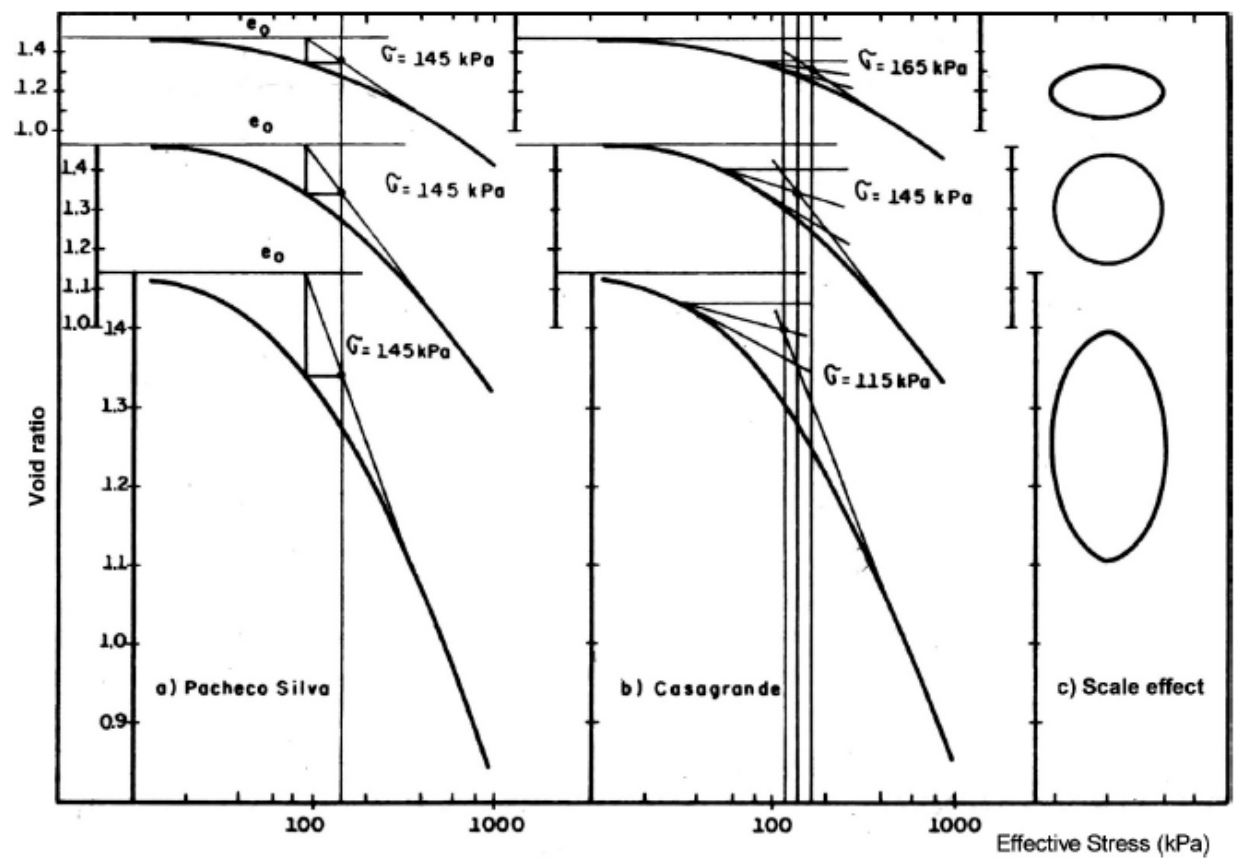

Figure 2-8: Influence of scale effect on determination of preconsolidation pressure for Casagrande construction method and Pacheco Silva (1970) method (Clementino, 2005)

\subsubsection{Peck Method}

This procedure is recommended for use on sensitive soil by Murthy (Murthy, 2003). The earliest description of this method found in the literature is by Peck et al. (Peck, Hanson, \& Thornburn, 1974), although they do not make any statement regarding the origin of the method. The method will thus be referred to as the Peck method. The steps for this method, shown in Figure 2-9, are as follows:

1. Determine the inflection point of the $e$-logp curve, where the slope begins to decrease

2. Draw a line tangent to the curve at this point and extend it upward to intersect the initial void ratio line

3. Find the intersection point of these two lines

The point $b$ corresponds to the pressure where structural breakdown takes place. In most extrasensitive clays, this point lies to the right of point a, the effective overburden pressure. It is suggested that the pressure increment between points $a$ and $b$ could represent the overconsolidation ratio of the soil, or it could represent physico-chemical bonds between soil particles. Stress increases due to construction should not exceed this increment. If it is exceeded, the stress condition will enter the steep branch of the curve and large settlements will be experienced (Peck, Hanson, \& Thornburn, 1974). 


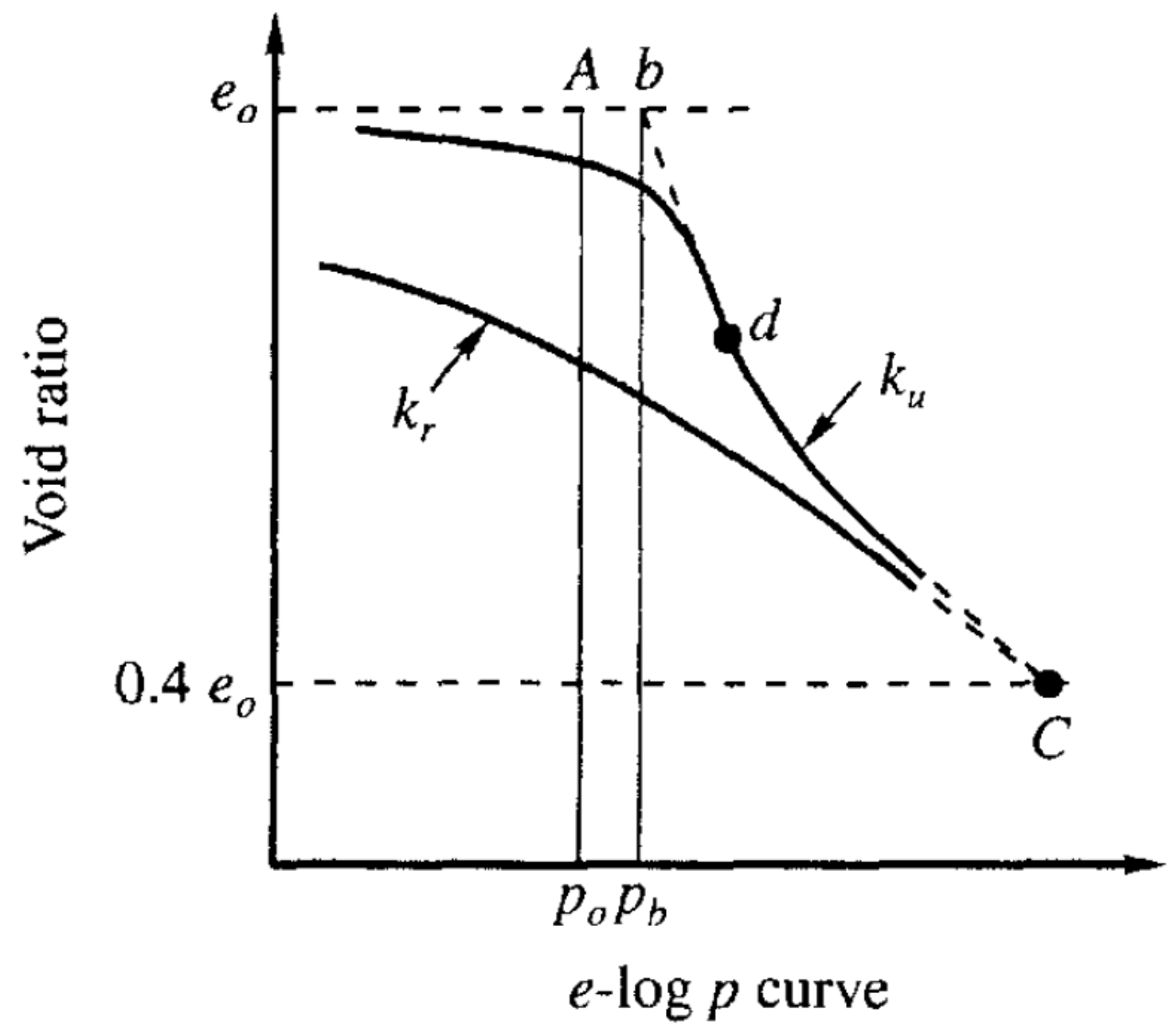

Figure 2-9: Determination of preconsolidation pressure using Peck method (Peck, Hanson, \& Thornburn, 1974)

\subsubsection{Pacheco Silva Method}

The procedure for this method, shown in Figure 2-10, is as follows (Clementino, 2005):

1. Draw a horizontal line at a value of $\mathrm{e}_{0}$.

2. Extend the virgin consolidation curve such that it intersects the initial void ratio line.

3. From the intersection of the two lines described, extend a straight vertical line such that it intersects the test curve.

4. From this point, draw a straight horizontal line to intersect the extension of the virgin consolidation curve.

5. The stress corresponding to the point identified in step 4 is defined as the preconsolidation pressure. 


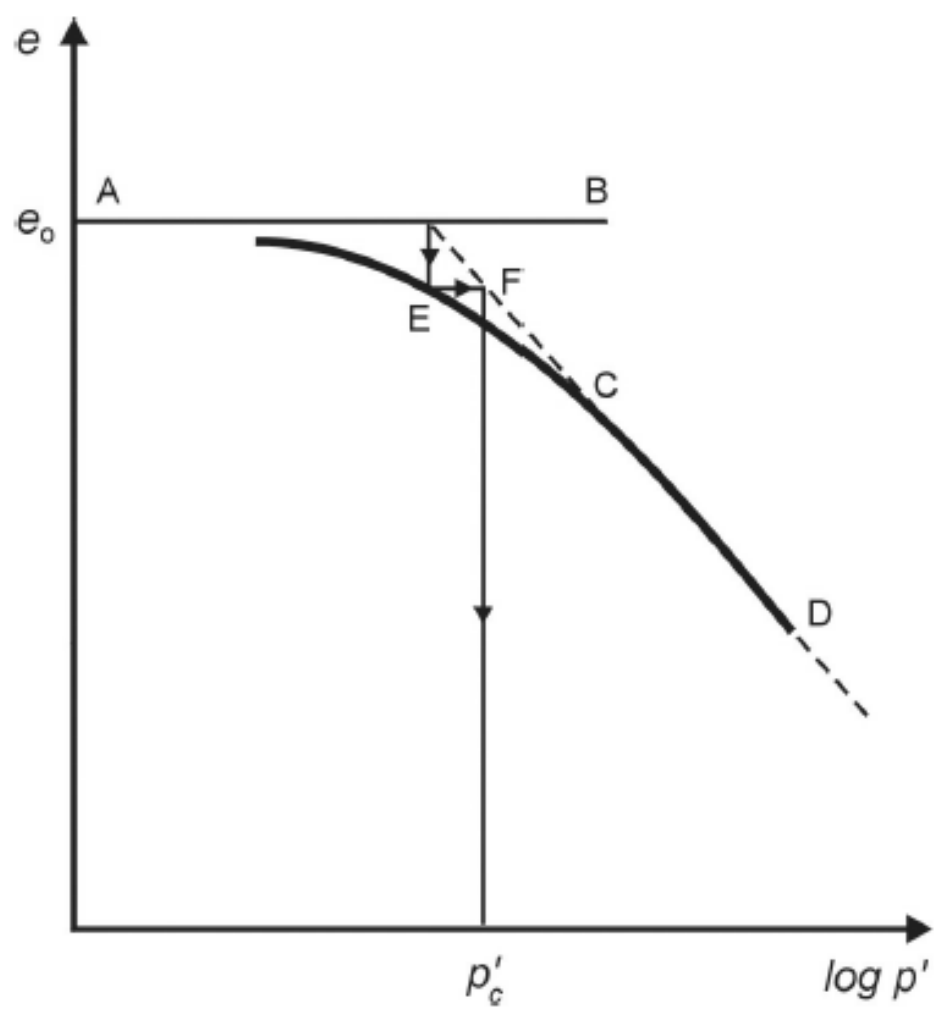

Figure 2-10: Determination of preconsolidation pressure using Pacheco Silva method (Clementino, 2005)

Clementino (2005) notes that the results of the Pacheco Silva method correlate well to the known preconsolidation pressure for four oedometer tests on reconstituted clay with low plasticity. The author mentions that, contrary to the Casagrande method, the Pacheco Silva method has the advantage of not being subject to scale effects.

The author also mentions that the Pacheco Silva method is an empirical method, and is used extensively in Brazil. Finally, the author suggests that this method can be used for comparison due to its simplicity.

It is not known whether or not Pacheco Silva in his original paper recommended that this procedure be used for sensitive clays. However, it is mentioned by Clementino (2005) that the method is easily applied to soft clays where the change from low compressibility to high compressibility becomes less clear due to sample disturbance.

As pointed out by Boone (Boone, 2010), the Pacheco Silva method has the limitation of being subject to user bias in determining the slope of the virgin compression curve. This is demonstrated in Figure 2-11. 


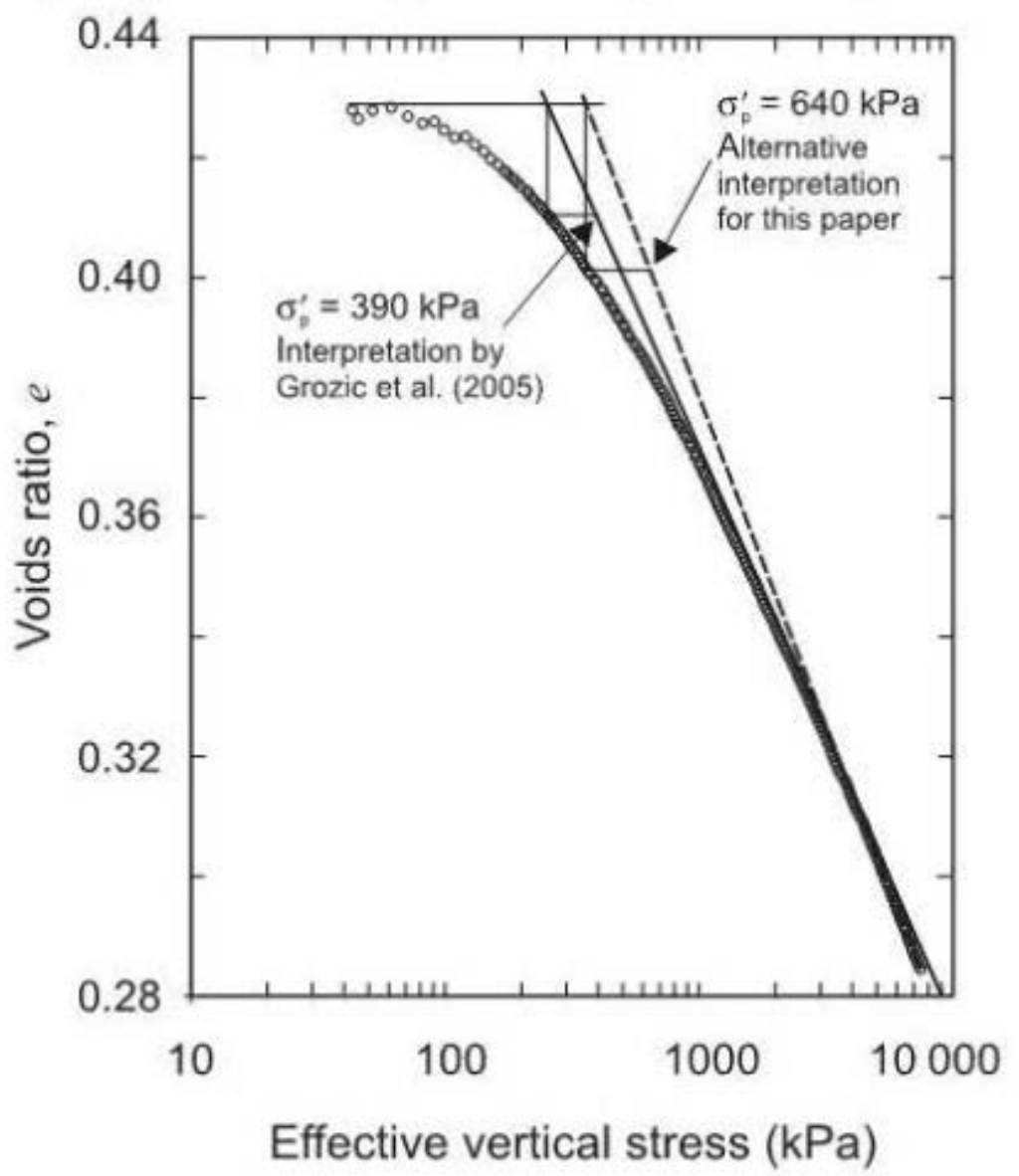

Figure 2-11: Example of consolidation curve with two possible interpretations using Pacheco Silva method (Boone, 2010)

\subsubsection{Nagarajet al. Method}

This method was introduced as a way to predict the field preconsolidation pressure accounting for sample disturbance. The procedure for using the method of this method is as follows (Nagaraj, Murthy, Vatsala, \& Joshi, 1990):

1) Perform a consolidation test on both an undisturbed and a completely remoulded sample of the same soil

2) Plot the results of both tests on a single $e$-logp plot

3) Obtain the point of maximum curvature for the undisturbed sample

4) Draw a line that is perpendicular to the consolidation curve of the remoulded sample and passes through the point of maximum curvature of the undisturbed sample

5) Draw a horizontal line having the value of the initial void ratio

6) Where the two lines intersect is defined as the preconsolidation pressure 
This procedure is to obtain the field preconsolidation pressure, taking sample disturbance into consideration. The method is based on two observations. The first is that the consolidation curve of a sensitive soil is comprised of three zones: non-particulate (i.e. pre-yield); transitional (i.e. post-yield or collapse); and particulate. The second observation is that, as the disturbance of a soil sample increases, the consolidation curve flattens and gradually approaches that of a completely remoulded sample. As this occurs, the point of maximum curvature also becomes lower in both void ratio and stress, and a straight line can be drawn connecting the points of maximum curvature for samples of varying levels of disturbance for the same soil. This line will be perpendicular to each consolidation curve at the point of maximum curvature. The authors then state that, logically, this line will also be perpendicular to the consolidation curve of a fully remoulded sample of the same soil. The effect of sample disturbance on a soil is shown on Figure 2-12:

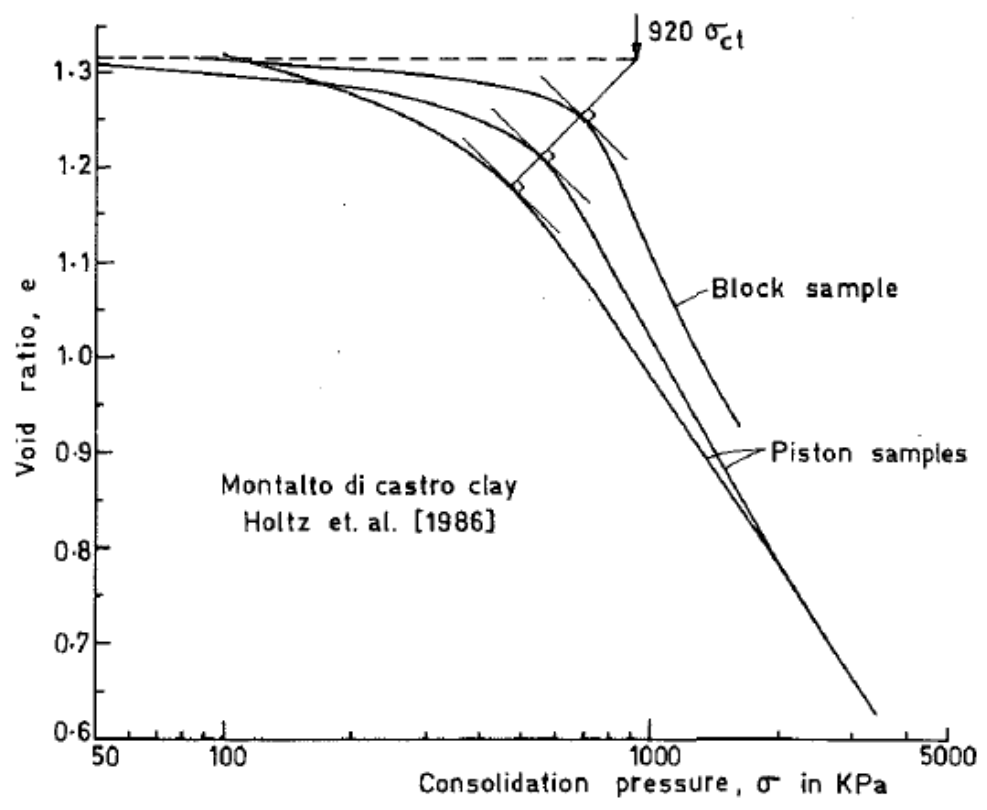

Figure 2-12: Effect of sample disturbance on e-logp curves (Nagaraj et al., 1990)

In their paper, Nagaraj et al. also propose a method for constructing the field consolidation curve for a sensitive soil. They argue that the method proposed by Schmertmann (1953) is not appropriate for sensitive soil because, due to cementation bonds, the slope of the consolidation curve prior to the preconsolidation pressure is much shallower than the slope of an unloading curve performed after the preconsolidation pressure is surpassed. This procedure is not described here, as it is outside the scope of the current study.

The figure below shows both the determination of field preconsolidation pressure by the method of Nagaraj et al., as well as their method for obtaining the field consolidation curve. 


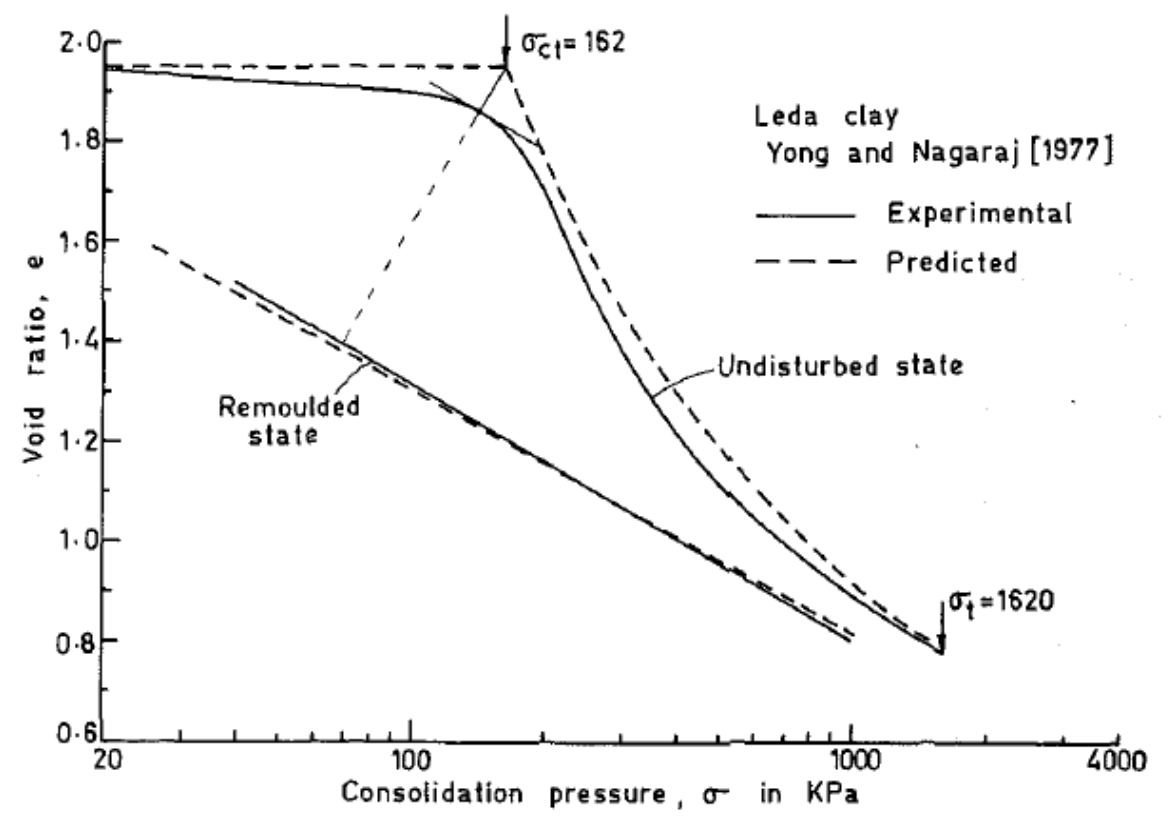

Figure 2-13: Determination of field preconsolidation pressure and field consolidation curve (Nagaraj, Murthy, Vatsala, \& Joshi, 1990)

The authors also propose a method for obtaining the yield stress of a sample of sensitive soil for the case where a consolidation test on an undisturbed sample is not available but the field vane shear strength $\left(\mathrm{S}_{\mathrm{u}}\right)$ is. After performing regression analysis on data of published Canadian soils and using the published preconsolidation pressures, the authors found the following correlation:

$$
\sigma_{c}=2.86 S_{u}+28
$$

The above formula has a correlation coefficient of 0.98 and a standard error of $30 \mathrm{kPa}$. The authors suggest that the error can be attributed to the fact that the consolidation samples have experienced sample disturbance, whereas the field vane shear values are not subject to sample disturbance. After performing their own proposed method to find the field preconsolidation pressure, the authors obtained the following correlation:

$$
\sigma_{c}=3.78 S_{u}+7
$$

This new formula has a correlation coefficient of 0.994 and a standard error of $8 \mathrm{kPa}$.

\subsubsection{Work Method}

The method proposed by Becker et al. is based on the principal of work (Becker, Crooks, Been, \& Jeffries, 1987). This is also known as total strain energy or energy per unit volume. 
Throughout this paper, it will be referred to as the work method, in keeping with common usage. It involves the calculation of cumulative total strain energy by means of the following equation:

$$
W=E=\sum_{i=1}^{n} 0.5\left(\varepsilon_{i+1}-\varepsilon_{i}\right)\left(\sigma_{i+1}^{\prime}+\sigma_{i}^{\prime}\right)
$$

where $\varepsilon$ and $\sigma^{\prime}$ are strain and effective stress at a given load increment, respectively. The procedure to determine preconsolidation pressure using the work method is as follows:

1) Calculate total strain energy for each load increment using the equation above

2) Plot work vs. effective stress on linear axes $(W-p)$

3) Identify the approximately linear portions of the $W-p$ plot

4) The stress corresponding to the intersection of these two straight lines is defined as the preconsolidation pressure

The work method is illustrated in Figure 2-14.

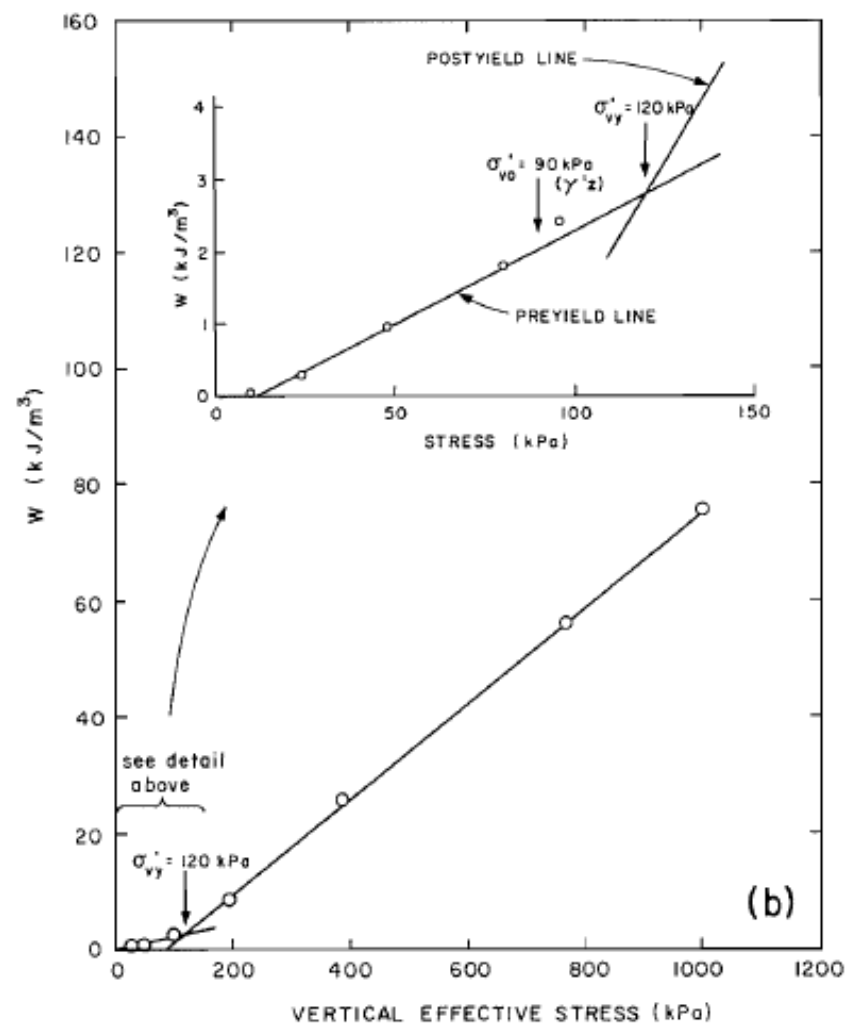

Figure 2-14: Determination of preconsolidation pressure using word method (Becker, Crooks, Been, \& Jeffries, 1987)

In a discussion paper, $\mathrm{Li}$ provides the following observations $(\mathrm{Li}, 1989)$. The first is that, if there is a linear relationship in the $W$-p plot, it implies that there is similarly a linear relationship in $e$ - 
logp plot. This is proven mathematically in Li's discussion. He then suggests that, based on this observation, there is no theoretical advantage to using the method proposed by Becker et al. (1987) over using the $e$-logp method.

The second observation made by Li (1989) is that a line that appears curved can be made to appear straighter by choosing either a different size for the graph on which the data is plotted, or by choosing different relative scales for the two axes. This introduces ambiguity and user bias into the work method. He suggests using the coefficient of correlation, R, to objectively measure the straightness of the line connecting the data points. The rationale for using this measure is that it is not affected by the selection of scale, and thus it is not affected by user bias.

\subsubsection{Wang and Frost Method}

This method is based on the concept of dissipated strain energy, as opposed to the work method, which is based on total strain energy (Wang \& Frost, 2004). The steps to perform this method, shown in Figure 2-15, are as follows:

1) Plot the total strain energy vs. effective stress on the axes E-p (or $W-p$ ), as outlined by Becker et al. (1987)

2) Extend the line TU so that it intersects the origin (i.e. at $0 \mathrm{kPa}$ ) and label the origin $\mathrm{O}$

3) Draw the horizontal axis OP'

4) Draw the line OE starting at the origin such that it has the same slope as the unloading/reloading line XY

5) Draw a vertical line at the location of $T$ (the final point of the last loading cycle) such that it intersects the horizontal axis at point $\mathrm{N}$

6) Draw point $\mathrm{D}$ such that the distance of TD is equal to the distance of EN

7) Connect points $\mathrm{O}$ and $\mathrm{D}$ with line $\mathrm{OD}$ to draw the dissipated strain energy line

8) The stress where the original axis Rp intersects the dissipated strain energy line is defined as the preconsolidation pressure

In the above figure, the line OE represents elastic strain energy, OD represents dissipated strain energy, OT represents total strain energy, and UR represents an unloading/reloading cycle. The proportion of total strain energy that is in fact dissipated strain energy can be known from the slope of the UR line.

Wang \& Frost (2004) comment that existing methods have the following drawbacks:

- Quantities such as total void ratio and total strain energy have not been associated with structural phenomena related to the consolidation process;

- They do not account for sample disturbance effects; and

- They are prone to operator bias, since they are all graphical methods. 


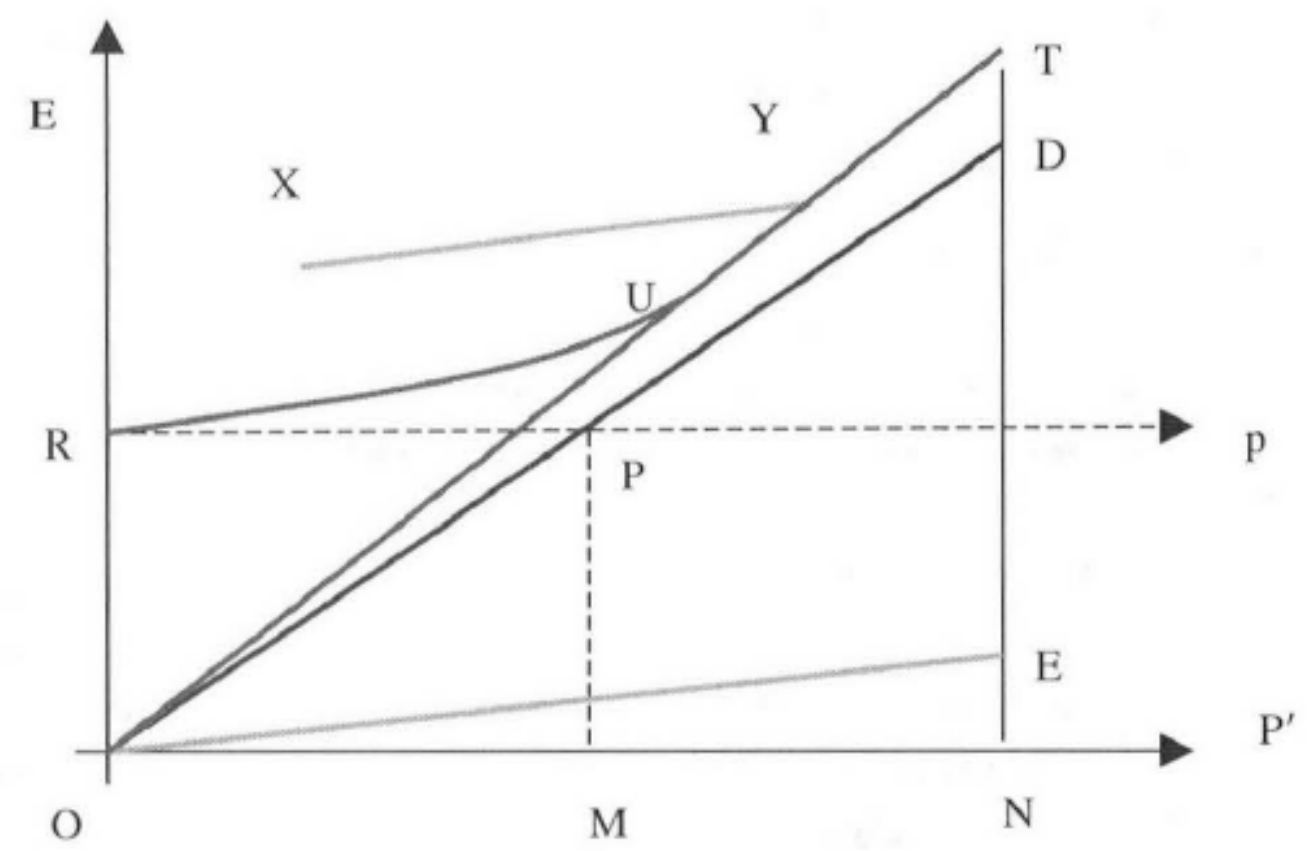

Figure 2-15: Determination of preconsolidation pressure using dissipated strain energy method (Wang \& Frost, 2004)

Unlike the method of Becker et al. (1987), which is based on total strain energy, the method of Wang and Frost (2004) is based on dissipated strain energy. The underlying concept is that total volume loss is equal to the summation of plastic and elastic volume loss.

$$
e^{T}=e^{p}+e^{e}
$$

where $\mathrm{e}^{\mathrm{T}}$ is total void ratio, $\mathrm{e}^{\mathrm{p}}$ is void ratio resulting from plastic deformation, and $\mathrm{e}^{\mathrm{e}}$ is void ratio resulting from elastic deformation. This relation is more familiar when considering that, due to the constant cross-sectional area of a consolidation ring, change in void ratio is equivalent to volumetric strain.

The graphical procedure described above can be avoided by using the equation:

$$
\sigma_{c}=\frac{O R}{\tan \left(D O P^{\prime}\right)}
$$


where $\sigma^{\prime}{ }_{c}$ is the preconsolidation pressure, OR is the distance between the origin and the point where the recompression line intersects the vertical axis, and DOP' is the angle between the unloading/reloading line and the horizontal axis.

According to the authors, the dissipated strain energy method has the advantage of having theoretical meaning, as it relates preconsolidation pressure to energy that is permanently stored in the form of dissipated strain energy.

The relationship between the Wang and Frost method and the work method can be visualized by modifying the above figure as shown in the figure below. The work method is shown in red.

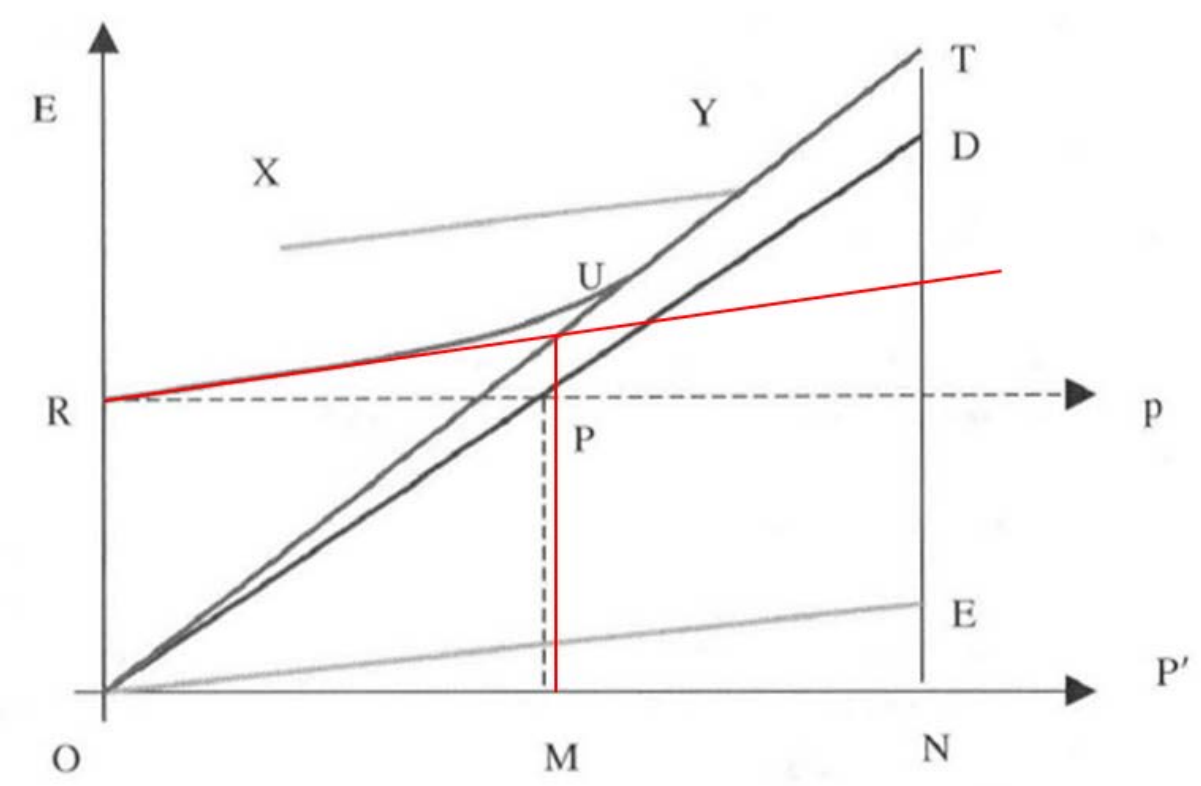

Figure 2-16: Relationship between Wang and Frost method and work method (modified from Wang \& Frost, 2004)

\subsubsection{Bilogarithmic Method}

The procedure for performing the bilogarithmic method is as follows:

1. Plot the results of a consolidation test on a bilogarithmic set of axes $(\log (1+e)-\log$, $\ln (1+e)-\ln \left(\sigma^{\prime}\right)$, or $\left.\ln (1+e)-\log p\right)$.

2. Approximate the pre-yield and post-yield parts of the curve as two straight lines.

3. The stress corresponding to the intersection of these straight lines is defined as the preconsolidation pressure.

The $\ln (1+e)-\ln p$ plot was proposed in 1979 as an improvement on the traditional $e$-logp plot (Butterfield, 1979). It was later proposed that a $\log (1+e)-\log$ plot be used (Oikawa, 1987), and finally a $\ln (1+\mathrm{e})$-logp plot was proposed (Onitsuka, Hong, Hara, \& Yoshitake, 1995). 
Traditionally, consolidation test data is plotted with void ratio on the vertical axis and effective stress plotted on the horizontal axis on a logarithmic scale (e-logp). The bilogarithmic method is based on the observation that the pre-yield and post-yield portions of the consolidation curve can more closely resemble a straight line if the vertical axis is plotted as a logarithm of $1+\mathrm{e}$.

Butterfield (1979) presented the following examples, among others, to validate this argument.
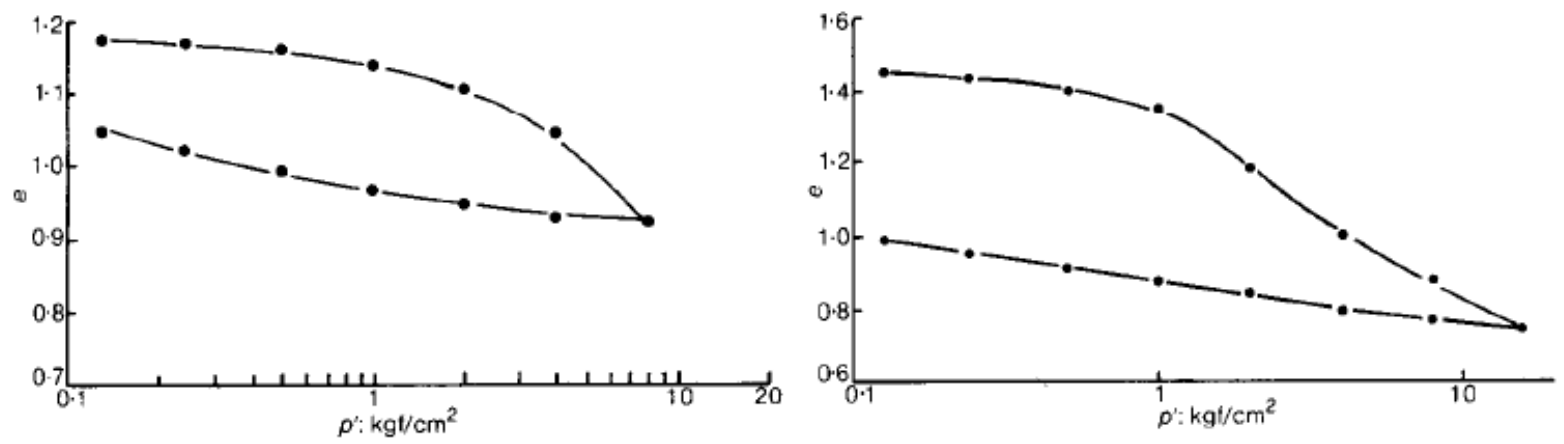

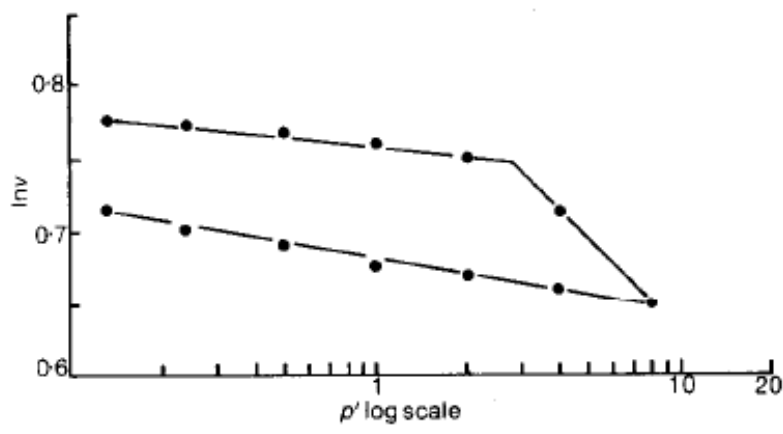

(a)

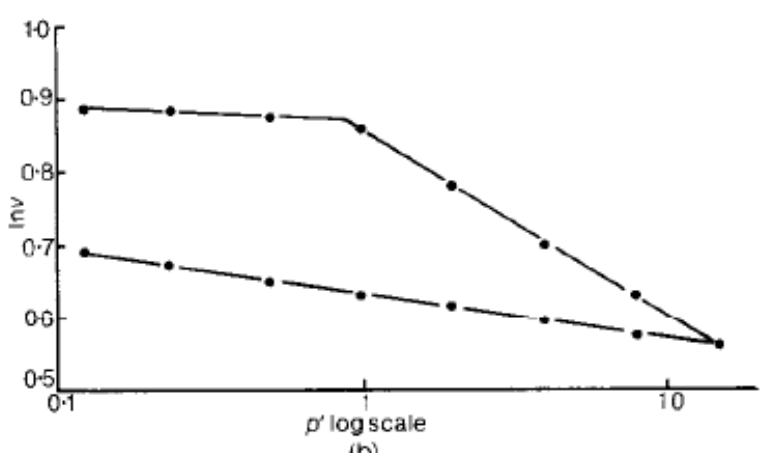

(b)

Figure 2-17: Consolidation data of some soils to demonstrate linearity of curves on bilogarithmic plot (Butterfield, 1979)

Multiple studies on reloading cycles where the maximum past pressure is known have found that the bilogarithmic method gives very accurate results (e.g. Umar \& Sadrekarimi, 2016).

It has been suggested that the value 1+e has more physical significance as the representing the total height of the specimen than simply using void ratio exclusively (Wang \& Frost, 2004).

In the present study, an additional bilinear method will be included for purposes of comparison with the bilogarithmic methods. Test data will be plotted on a traditional $e$-logp plot, and the preconsolidation pressure will be identified as the intersection of the lines that approximate the pre-yield and post-yield parts of the curve on that plot. Thus, the procedure for obtaining preconsolidation pressure is identical to that of the bilogarithmic methods, with the only difference being in which forms of void ratio and effective stress are plotted on the axes. This 
approach is not found anywhere in the literature, and is included here only to study the influence of plotting data on the different plots. This approach shall be referred to as the "e-logp bilinear" method.

\subsubsection{Sallfors Method}

To perform this method, axial strain and effective stress are both plotted on linear scales. The preyield and postyield parts are then extended with straight lines and the point of intersection of these two lines is located. Then, an isosceles triangle is inscribed such that the long edge touches the data curve. Figure 2-18 illustrates this method, where the point labelled B is the intersection of the straight line parts, and the point B' is the preconsolidation pressure (Sallfors, 1975).

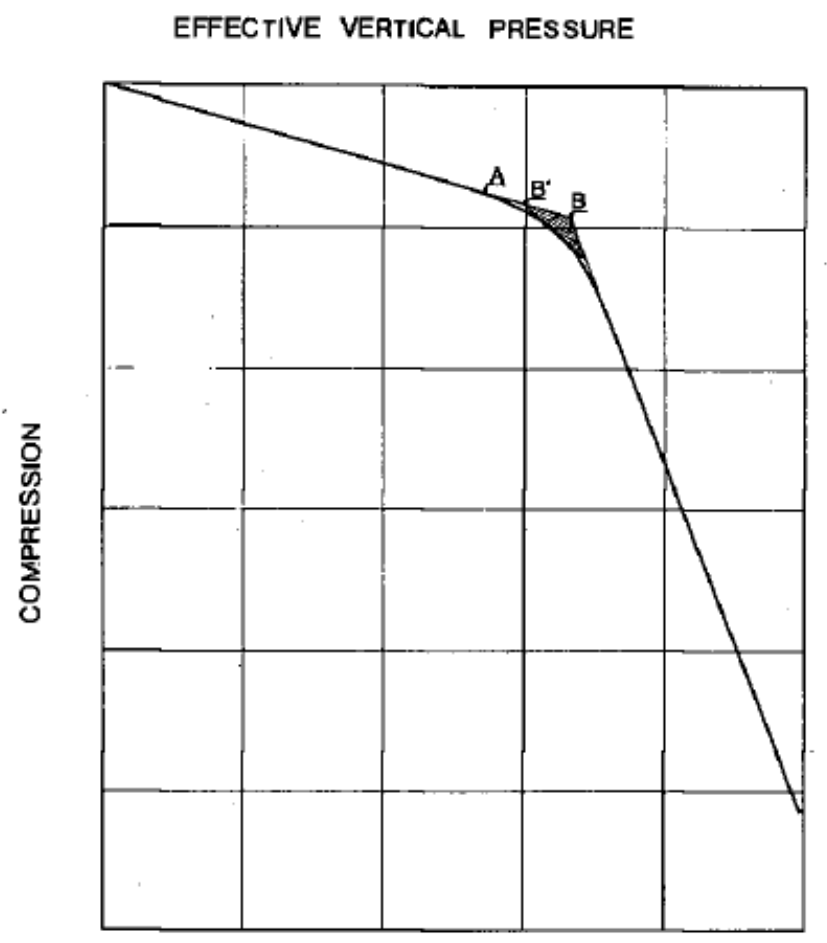

Figure 2-18: Determination of preconsolidation pressure using the Sallfors method (Sallfors, 1975)

According to Sallfors (1975), the point A where the curve first begins to bend represents the first yield of the sample; however, this point cannot be realistically used as a yield criteria because it is impossible to obtain a perfectly undisturbed sample. Furthermore, he asserts that both the Casagrande method and point B in Figure 2-18 are "too optimistic" (i.e. overpredict the preconsolidation pressure). As such, he proposes using point B', seemingly as a compromise between these other approaches. Due to slight scale effects, the standard practice is adopted by which a length of $1 \%$ on the strain axis corresponds to $10 \mathrm{kPa}$ on the effective stress axis (Larsson \& Sallfors, 1986). 
Little reference has been found in the literature to using the method proposed by Sallfors to determine the preconsolidation pressure of a soil. Investigators typically do not include it in their comparative studies. This method will nevertheless be used in this study for comparison with other established methods.

Interestingly, Sallfors (1975) mentions that the incremental loading consolidation test has a "serious drawback" that the consolidation curve is not fully defined. Rather, only a small number of points are obtained.

\subsubsection{Lanbu Method}

The method proposed by Janbu is based on the resistance concept (Paniagua, L'Heureux, Yang, \& Lunne, 2016). The method involves plotting the constrained modulus, D (also known as tangent modulus, $\mathrm{M}$, sometimes known as $\mathrm{E}_{\text {oed }}$ ) against effective stress, $\sigma^{\prime}$, both on a linear scale. However, Janbu did not specify the procedure that should be followed based on this plot (Paniagua, L'Heureux, Yang, \& Lunne, 2016). As such, two variations of the Janbu methods] have been found in the literature. The first is as follows:

1. Plot constrained modulus vs. effective stress on linear scales

2. Determine the minimum value of $\mathrm{D}$ and draw a horizontal line

3. Determine the slope of the approximately linear part of the descending part of the curve and extend it downward to intersect with the previously drawn horizontal line

4. The point where the two lines intersect is defined as the preconsolidation pressure

This method is demonstrated in the figure below.

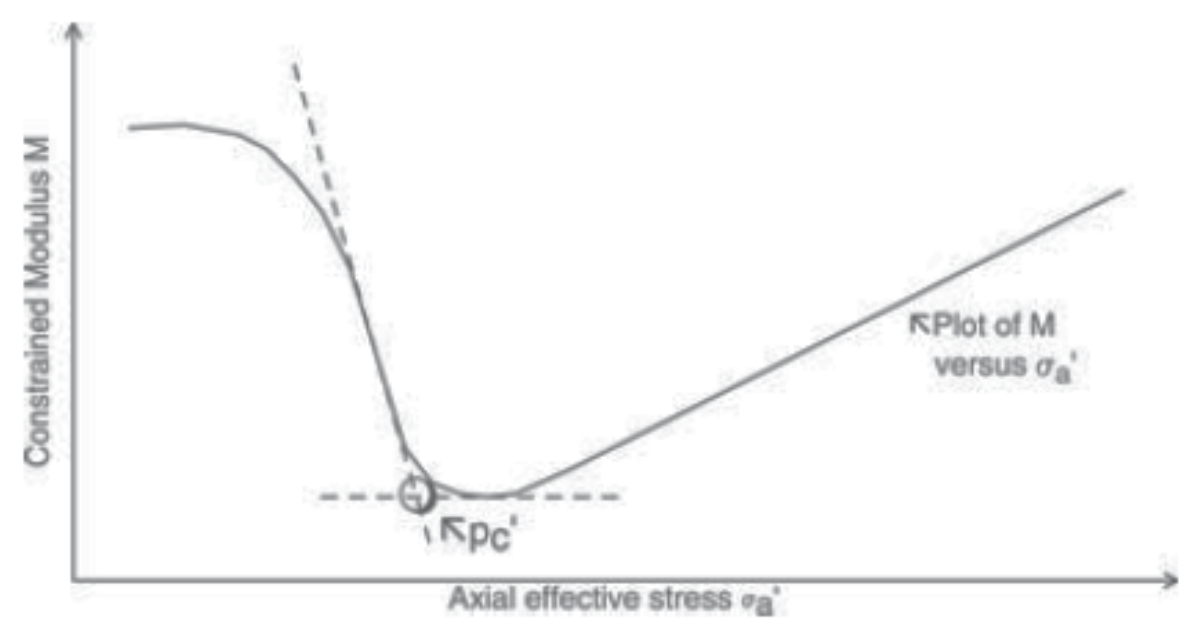

Figure 2-19: First variation of Janbu's method for determining preconsolidation pressure (Lunne, Berre, Andersen, Sjursen, \& Mortensen, 2006) 
The second variation of the Janbu method is described below. This variation of the method has been used by Yoon et al. (2011) and by Chung and Singh (2015). This is the variation of the method that will be used in this study.

1. Plot constrained modulus vs. effective stress on linear scales

2. Determine the slopes of the descending and ascending parts of the $D-p$ plot

3. The point where the two lines intersect is defined as the preconsolidation pressure

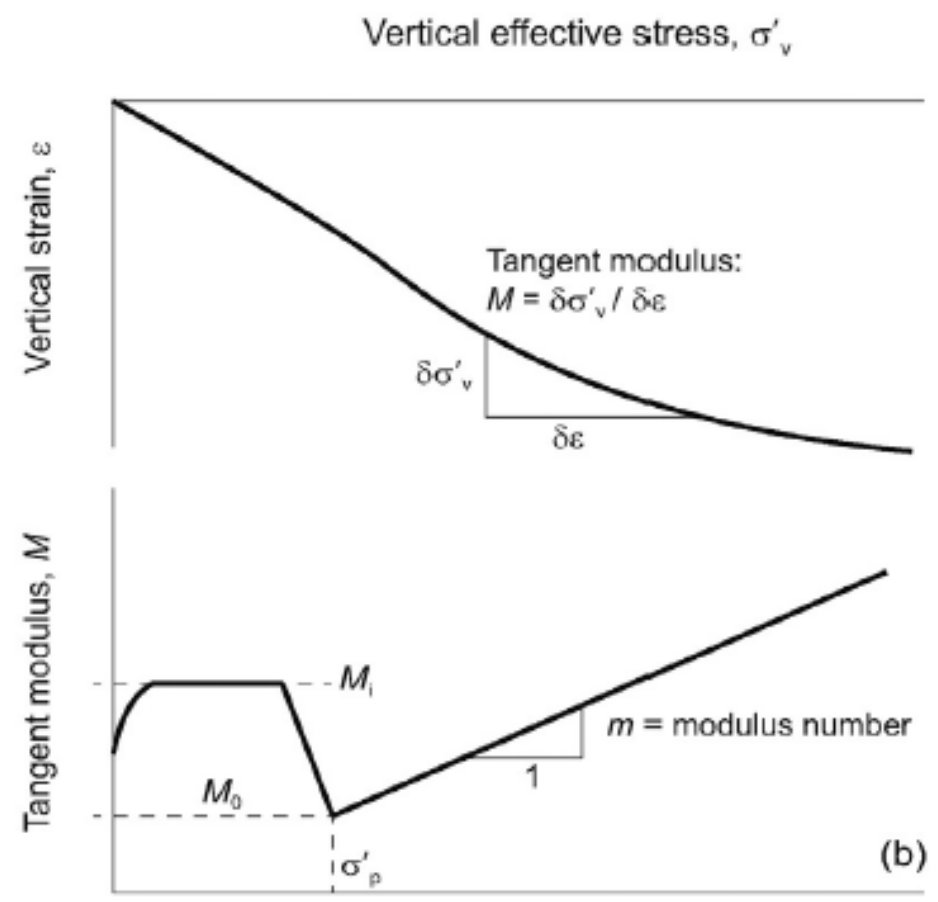

Figure 2-20: Second variation of Janbu's method for determining preconsolidation pressure (Chung and Singh, 2015)

Figure 2-21 presents the concept as first introduced by Janbu. 


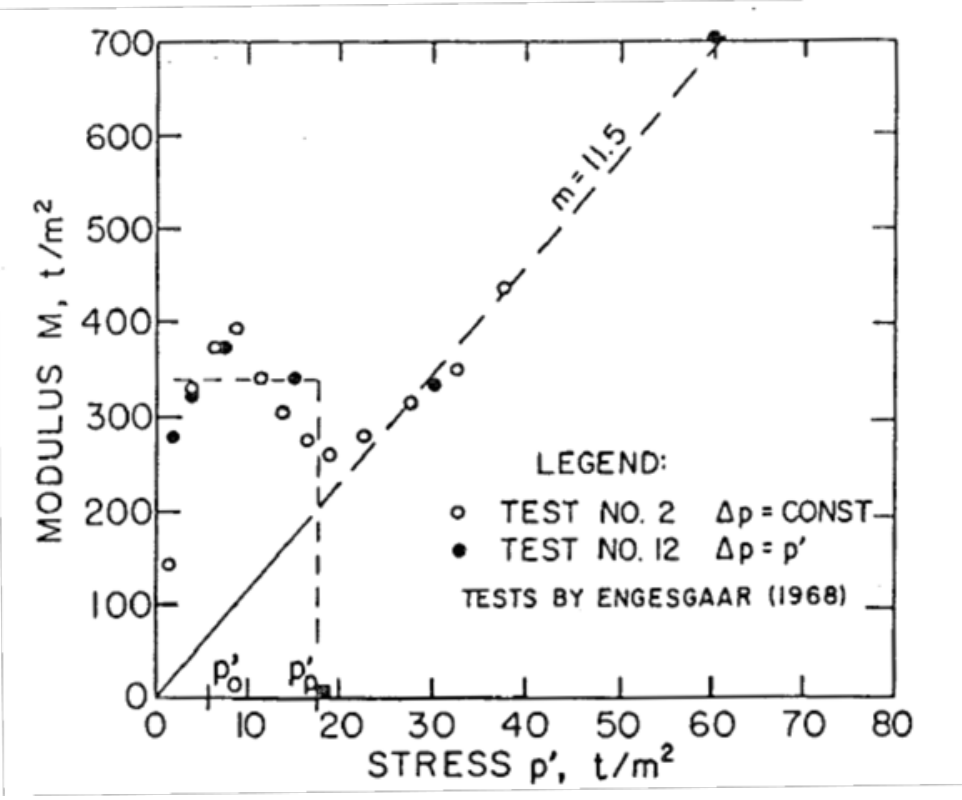

Figure 2-21: Modulus vs. stress curve for two overconsolidated, very sensitive clay samples (Janbu, 1969)

In a later paper, Janbu notes that the shape of the $D$-p curve varies depending on the soil type (Janbu, 1981). Overconsolidated soils tend to have a constant value for $\mathrm{m}_{\mathrm{v}}$ between $\mathrm{p}_{0}{ }_{0}$ and $\mathrm{p}_{\mathrm{c}}$, and then it increases past $\mathrm{p}_{\mathrm{c}}$. Normally consolidated soil tends to have $\mathrm{M}$ increasing linearly with stress when p'>p'c. Normally consolidated quick clay, such as that being studied presently, shows a drop in $\mathrm{M}$ around $\mathrm{p}_{\mathrm{c}}$, and then it increases non-linearly with an increasing slope. Janbu (1981) attributed this drop to a "collapse of the metastable clay skeleton in the quick clays". These tendencies are shown in the figure below.
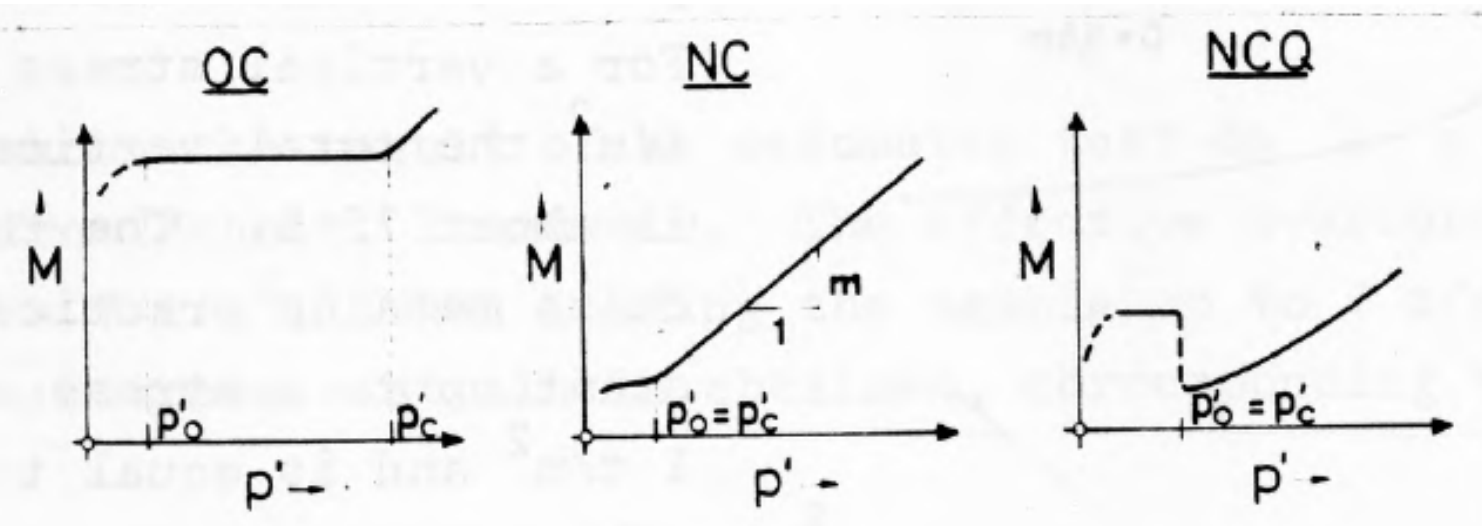

Figure 2-22: Typical mv vs. p’ curves for some soil types. OC = overconsolidated, NC = normally consolidated, and NCQ = normally consolidated quick clay (Janbu, 1981) 
According to Becker et al. (1987), the method proposed by Janbu is effective for soils with a well-defined break in the region surrounding p'c, but less suitable for soils with more rounded curves. Grozic et al. (2003) also state that Janbu's method is mostly recommended for use on soils with high sensitivity and low OCR.

\subsubsection{Karlsrud Method}

The method of Karlsrud is, similarly to that of Janbu (1969), based on the M vs. p plot, but a different interpretation of the graph is used. The steps for the method of Karlsrud are as follows (Karlsrud \& Hernandez-Martinez, 2013):

1. Identify the point on the before yielding where the tangent modulus begins to decrease and the corresponding effective stress.

2. Identify the point where the tangent modulus begins to increase along the virgin modulus line and the corresponding effective stress.

3. Calculate the average of the two values of effective stress obtained in the previous steps. This is defined as the preconsolidation pressure.

This method is shown graphically in the figure below.

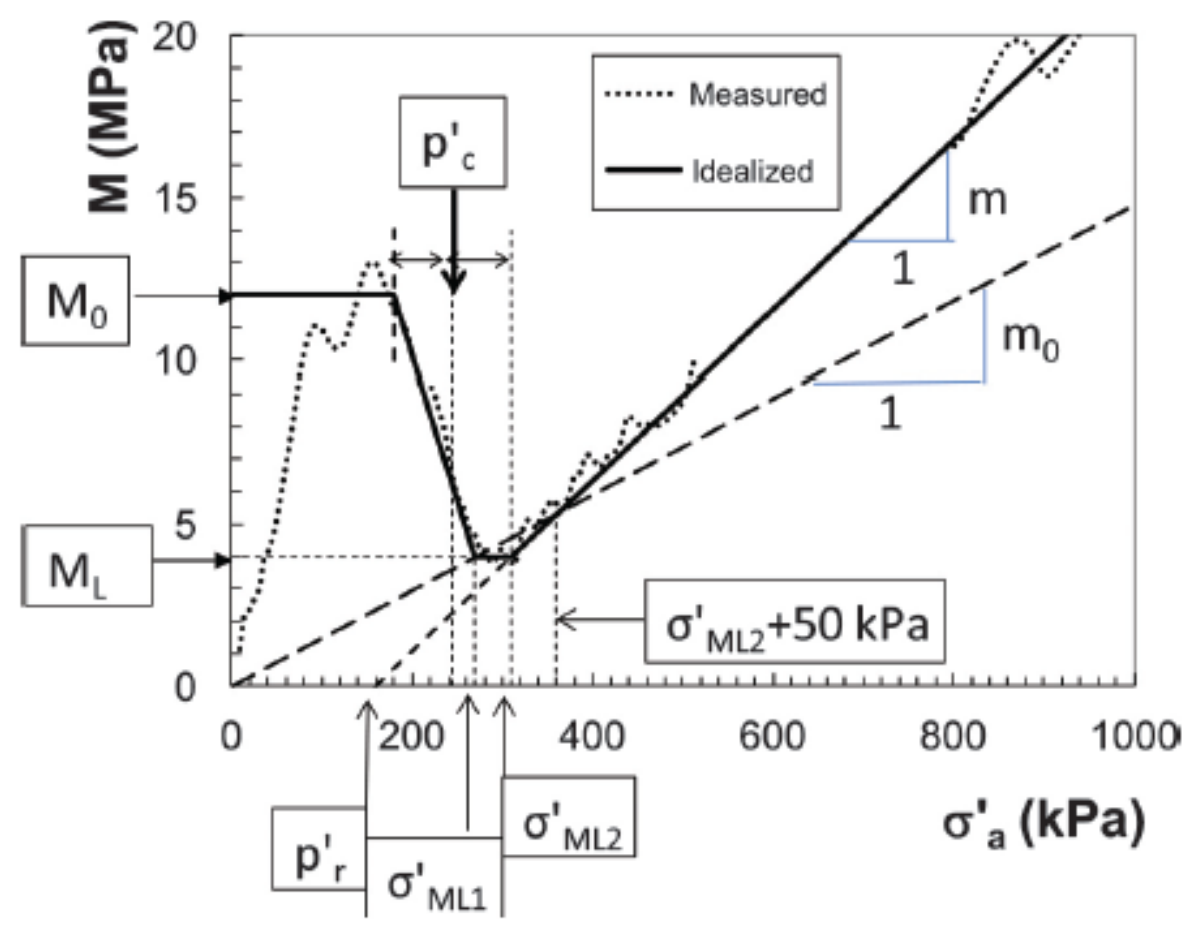

Figure 2-23: Determination of preconsolidation pressure by Karlsrud's method (Karlsrud \& Hernandez-Martinez, 2013) 
According to Karlsrud and Hernandez-Martinez (2013), this method compares very closely with the methods of Casagrande (1936), Becker (1987), and another unnamed curvilinear method for the determination of preconsolidation pressure. More specifically, the result of this method equalled approximately the average of these three other methods. They state that a benefit of using this method is its simplicity.

\subsubsection{Burland Method}

In his 1990 lecture, Burland proposed a new parameter called the void index, $\mathrm{I}_{\mathrm{v}}$, based on the consolidation curve of a resedimented soil sample. The purpose of the void index is to normalize the consolidation curve with respect to the void ratio (Burland, 1990).

In the same lecture, Burland proposed a new method for determining the preconsolidation pressure of a soil, based on this parameter. The procedure is as follows:

1) Perform a consolidation test on a resedimented soil sample to obtain the intrinsic compression line (ICL).

2) Obtain the void ratio corresponding to $100 \mathrm{kPa}$ and $1000 \mathrm{kPa}\left(e_{100}^{*}\right.$ and $e_{1000}^{*}$ respectively) for the resedimented sample.

3) Perform a consolidation test on a sample of undisturbed soil.

4) Calculate void index for each void ratio in the consolidation test:

$$
I_{v}=\frac{e-e_{100}^{*}}{e_{100}^{*}-e_{1000}^{*}}=\frac{e-e_{100}^{*}}{C_{c}^{*}}
$$

5) Plot $I_{v} v s . \log \left(\sigma^{\prime}\right)$ for the consolidation test.

6) The stress corresponding to the intersection of two approximate straight lines on the $I_{v^{-}}$ $\log \left(\sigma^{\prime}\right)$ plot is defined as the preconsolidation pressure.

If no resedimented sample test data is available, the following empirical relations can be used:

$$
\begin{gathered}
e_{100}^{*}=0.109+0.679 e_{L}-0.089 e_{L}^{2}+0.016 e_{L}^{3} \\
C_{c}^{*}=0.256 e_{L}-0.04
\end{gathered}
$$

where $e_{\mathrm{L}}$ is the void ratio at the liquid limit.

The two equations above are based on regression for a large number of tests. These equations should only be used for values of $e_{\mathrm{L}}$ that fall in the range between 0.6 and 4.5 (i.e. liquid limit between 25 and 160), and they should only be used for soils whose Atterberg limits fall above the A-line of a plasticity chart (Burland, 1990). 
The shape of the consolidation curve obtained using Burland's method is expected to look similar to the e-logp curve, since void index is simply a manipulation of void ratio (Grozic, Lunne, \& Pande, 2003).

\subsubsection{Boone Method}

The procedure to determine preconsolidation pressure by Boone's method is as follows (Boone, 2010):

1) Plot the consolidation data on an e-logp curve.

2) Determine the current overburden pressure and obtain the corresponding point on the $e$ logp curve $\left(\sigma^{\prime}{ }_{\mathrm{v} 0}, \mathrm{e}_{\mathrm{v} 0}\right)$

3) Determine the recompression index $\left(\mathrm{C}_{\mathrm{r}}\right)$, defined as the average slope of the unload/reload cycle

4) Determine the maximum compression index $\left(\mathrm{C}_{\mathrm{c}, \max }\right)$

5) Determine the load increment where the compression index is a maximum, $\left(\sigma_{v, \max }, \mathrm{e}_{\mathrm{v}, \min }\right)$

6) Calculate the intercepts on the void ratio axis of the equations:

$$
\begin{gathered}
e_{c}=C_{c, \text { max }} * \log \sigma_{v, \text { max }}^{\prime}+e_{\text {min }} \\
e_{r}=C_{r} * \log \sigma_{v, 0}^{\prime}+e_{v, 0}
\end{gathered}
$$

7) Once the slope and intercept of both lines are known, calculate their intersection on the $e$ logp scale:

$$
e_{p}=\left(\frac{e_{c}}{C_{c, \max }}-\frac{e_{r}}{C_{r}}\right) /\left(\frac{1}{C_{\max }}-\frac{1}{C_{r}}\right)
$$

8) Calculate the preconsolidation pressure:

$$
\sigma_{p}^{\prime}=10^{\left(\frac{e_{c}-e_{p}}{c_{c, \max }}\right)}
$$

Boone (2010) recommends obtaining the recompression index at a low unloading stress, as the value of recompression index has been found to be larger when the stress at unloading is larger. The slope of the unloading curve is used instead of the slope of the initial branch of the recompression curve in order to minimize the influence of sample disturbance. 


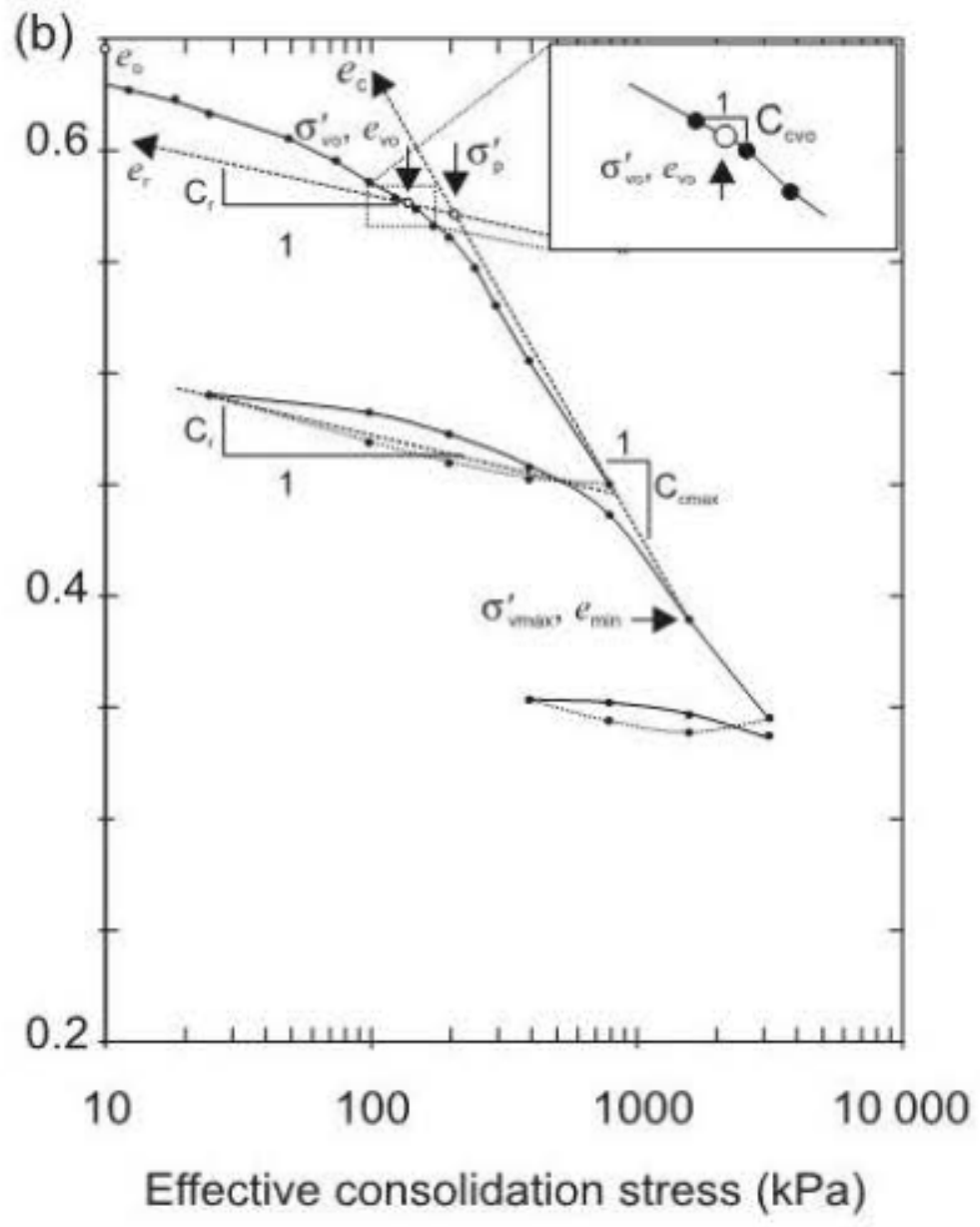

Figure 2-24: Determination of preconsolidation pressure by Boone’s method (Boone, 2010)

It should be noted that $e_{\mathrm{v} 0}$ as used by Boone (2010) does not refer to the void ratio before consolidation, but rather to the void ratio at the current overburden pressure.

It appears to be quite novel to describe the pre-yield and post-yield lines in terms of linear equations in the manner of Boone (2010) rather than purely graphically. The author mentions that performing the operation mathematically avoids graphical interpolation on a logarithmic scale.

\subsubsection{Lacobsen Method}

The preconsolidation pressure of Jacobsen (1992) is defined as:

$$
p^{\prime} c=2.5 * \sigma_{K}^{\prime}
$$

Eq. $2-14$ 
where $\sigma^{\prime}{ }_{K}$ is the effective stress at the point of maximum curvature. This method is based on Jacobsen's observations of Danish overconsolidated clay (Grozic, Lunne, \& Pande, 2003). It is not mentioned whether or not this method is suitable for sensitive clay.

\subsubsection{Minimum Pore Pressure Ratio Method}

The idea of defining preconsolidation pressure as the stress corresponding to the minimum pore pressure ratio obtained during the test was first introduced by Seah and Juirnarongrit (2003). Pore pressure ratio is calculated as the division of base excess pore pressure by total axial stress:

$$
R=\frac{\Delta u}{\sigma_{a}}
$$

The results upon which the development of this method is based were obtained using CRS tests with radial drainage (Seah \& Juirnarongrit, 2003). In fact, this method is well-suited to CRS tests, in which the pore pressure ratio is continuously measured. This method is illustrated in Figure 2-25.

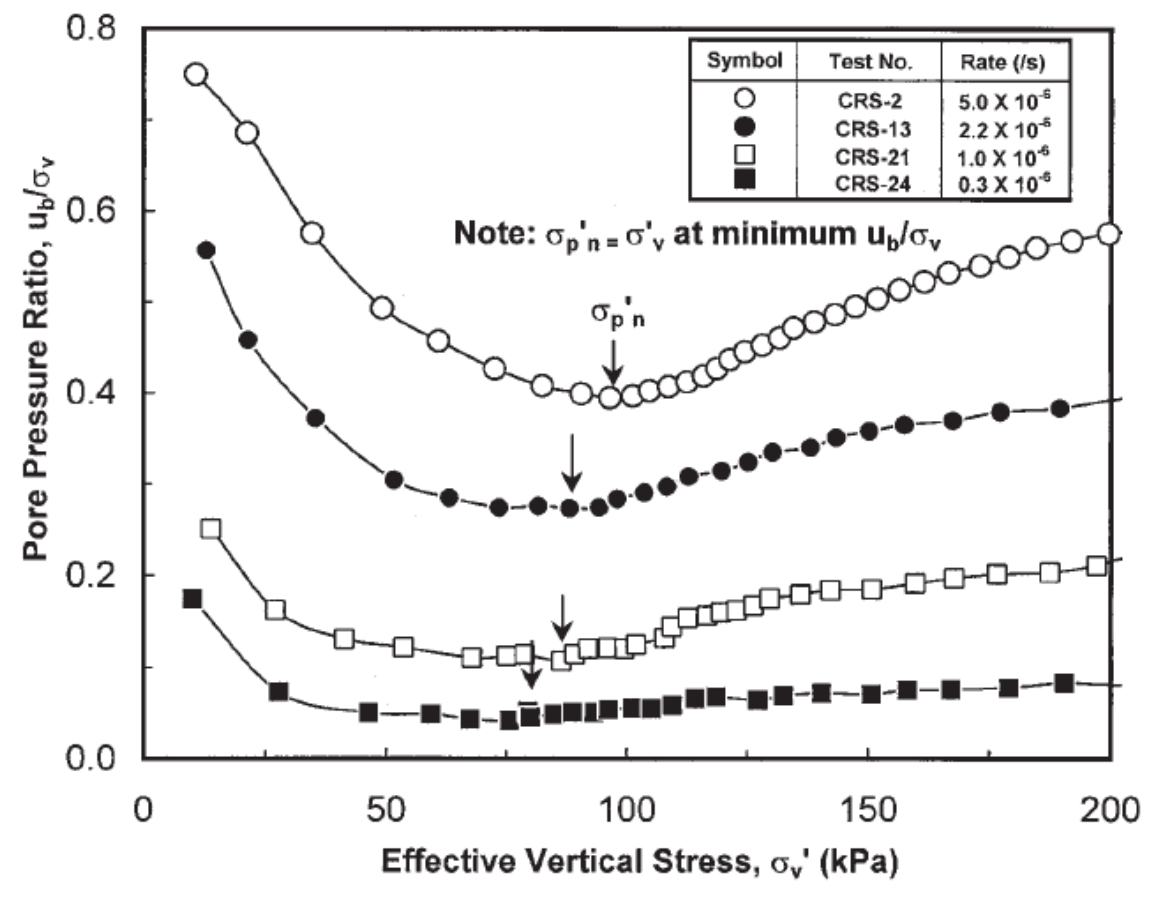

Figure 2-25: Use of minimum pore pressure ratio for determination of preconsolidation pressure (Seah \& Juirnarongrit, 2003)

The method has also been applied to CRS tests using vertical drainage (DeGroot, Landon, \& Ryan, 2007). They observed that this point corresponds well with the preconsolidation pressure 
as determined by other methods. Thus, they suggest that the stress corresponding to the minimum pore pressure ratio can be interpreted as the preconsolidation pressure. Defining preconsolidation pressure based on this criterion removes the need to perform graphical operations. Furthermore, there is no need to interpret effective stress on a logarithmic scale. The authors report that the result obtained using this method matches very closely with the preconsolidation pressure obtained using the work method, as shown in the figure below.

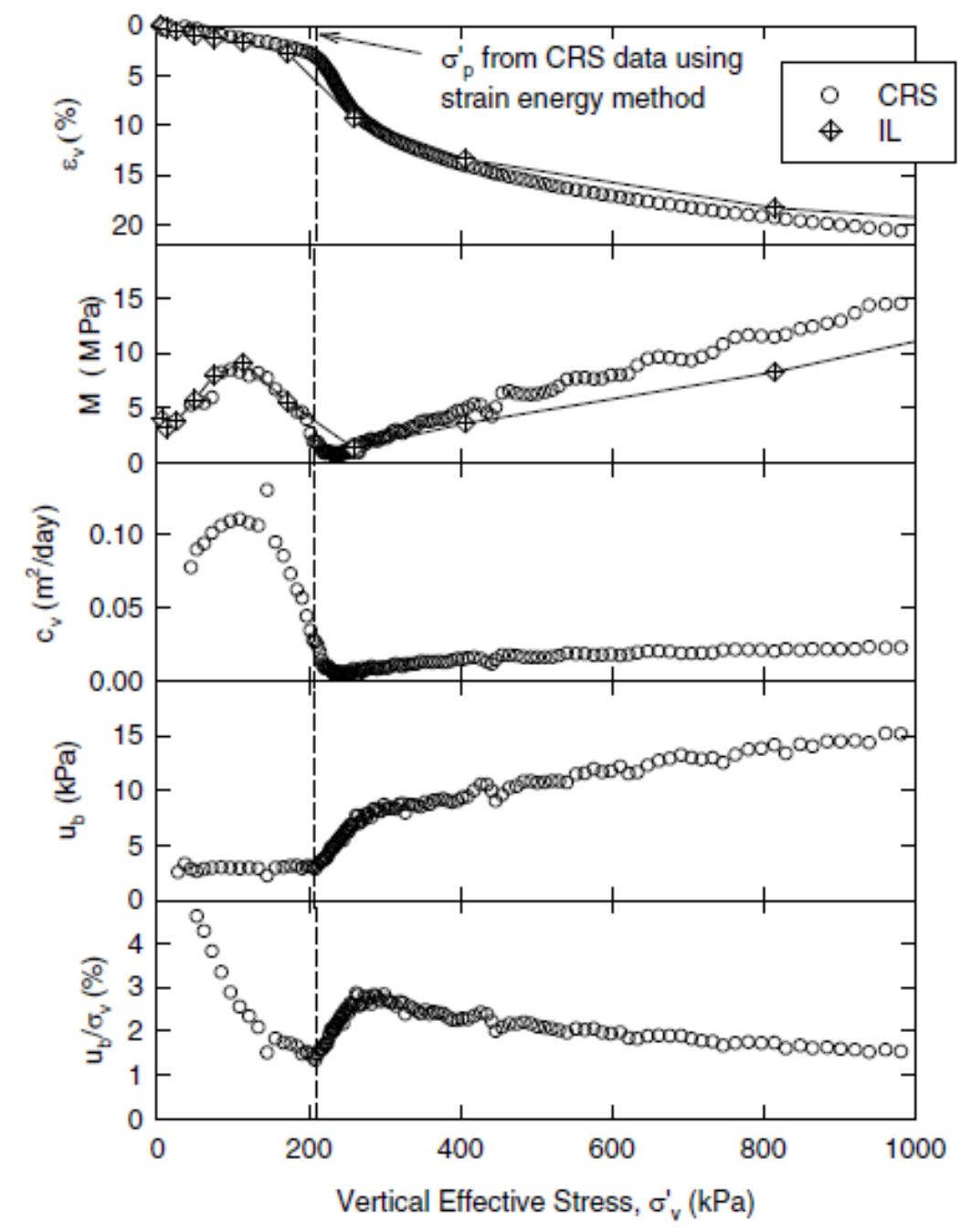

Figure 2-26: Comparison of minimum pore pressure ratio with work method and other parameters (DeGroot et al., 2007)

\subsubsection{Yoon et al. Method}

This method is based on shear wave velocity at small strain (Yoon, Lee, Kim, \& Lee, 2011). It is based on the fact that, in the system of soil particles and pore water, shear waves only travel through soil particles. Thus, the behaviour of shear waves in soil is a function of effective stress. 
To obtain the necessary data, it is required to equip a standard consolidation cell with bender elements. A schematic of such an oedometer cell is shown in Figure 2-27.

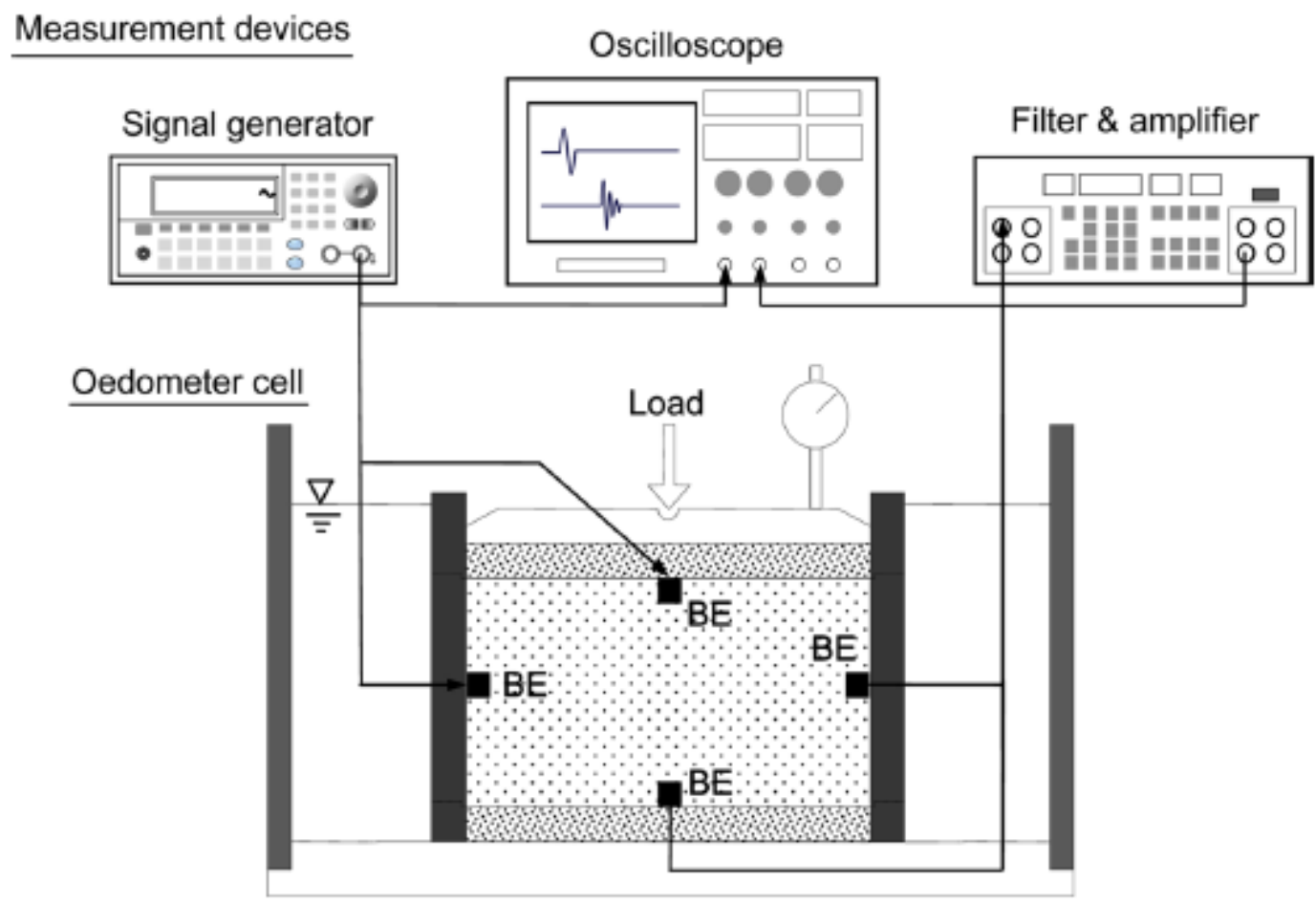

Figure 2-27: Instrumented oedometer cell with for measuring shear waves (Yoon et al., 2011)

During the consolidation test, a loading increment ratio of approximately 1 is used. Each loading step lasts a sufficient amount of time to allow all excess pore pressure to dissipate. Vertical settlement and shear waves are measured when settlement does not proceed after the dissipation of pore pressure.

The shear wave velocity in terms of effective stress is calculated by the following formula.

$$
V s=\alpha\left(\frac{\sigma_{p}^{\prime}+\sigma_{m}^{\prime}}{2}\right)^{\beta}=\alpha\left(\sigma_{o}^{\prime}\right)^{\beta}
$$

In the above formula, $\alpha$ and $\beta$ are experimentally determined and $\sigma^{\prime}{ }_{0}$ is the average effective stress. The $\beta$ parameter increases with decreasing soil density. This parameter increases after preconsolidation pressure is exceeded. Figure 2-28 presents the determination of preconsolidation pressure from shear wave test data. 


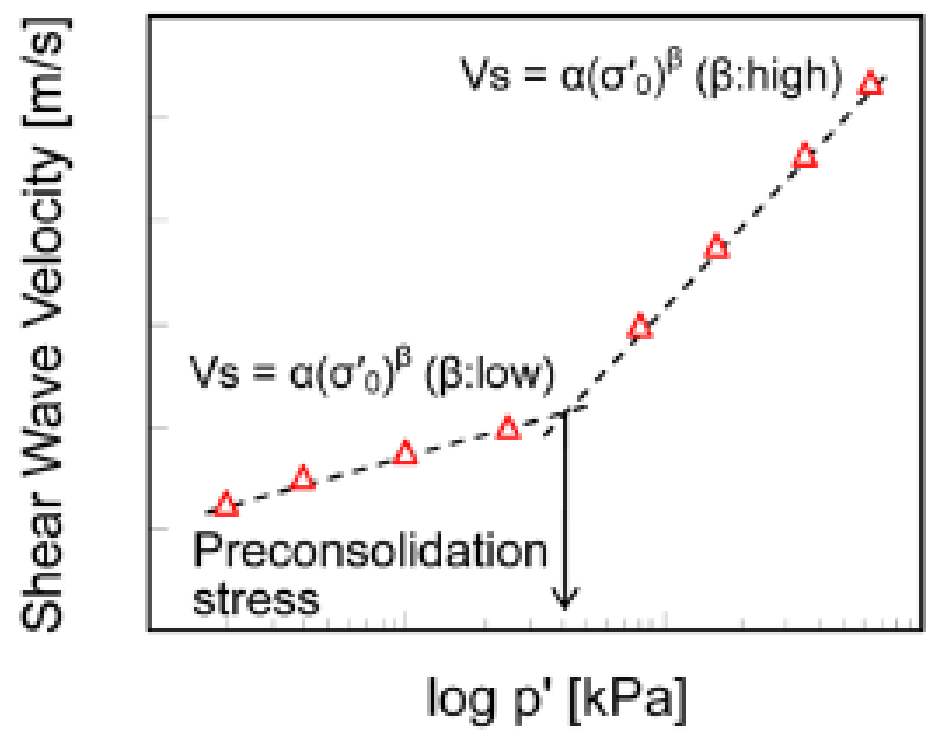

Figure 2-28: Determination of preconsolidation pressure by the Yoon et al. method (Yoon et al., 2011)

\subsection{Previous Studies on Performances of Different Methods}

Previous studies have compared methods of determining preconsolidation pressure for different soils from consolidation test data.

In 2003, Grozic et al. performed a comparison study of five graphical methods on soil with low plasticity (Grozic, Lunne, \& Pande, 2003). They note that the Casagrande and Janbu methods, which are commonly used in engineering practice, are both based on the assumption that soil experiences a change in stiffness (from more to less stiff) around the preconsolidation pressure. They additionally note that sample disturbance effects make it difficult to reliably determine the preconsolidation pressure of overconsolidated soil with a low plasticity using the oedometer test.

In their study, Grozic et al. (2003) assigned each method a value from 1 to 5 indicating the level of difficulty of determining preconsolidation pressure using the given method. This subjective value does not say anything about the accuracy of the method, but only about its ease of use. They found that the most difficult method to use was that of Janbu. The remaining methods listed in order of decreasing difficulty are the Casagrande, work, Burland, and bilogarithmic methods. They noted that the inclusion of more data points by using smaller load increments in the oedometer test would make interpretation easier.

The study of Grozic et al. (2003) was on overconsolidated glaciomarine clays, and their recommendation is that the bilogarithmic method should be used for this soil type. They found that the Janbu method gave the highest results and the work method gave the lowest results. The 
version of the Janbu method used by Grozic et al. (2003) included a straight line at the minimum value of constrained modulus. They found that the Janbu method was very difficult to use due to the high OCR and low plasticity of the soil used in their study. They also found that the bilogarithmic method gave similar results to the Casagrande method.

More recently, two studies were performed independently, which are reported in different conference proceedings.

Paniagua et al. (2016) do not draw a conclusion on which method is "correct", noting the comment by Becker et al. that the more important question is one of repeatability and lack of ambiguity. They do comment, however, that in practice, at least three different methods should be used and compared. The variation of Janbu's method used in this study was the same as that used by Grozic et al. (2003). The conclusions of the study by Paniagua et al. are summarized in Table 2-3.

Table 2-3: Conclusions of a previous study on graphical procedures to determine preconsolidation pressure (Paniagua, L'Heureux, Yang, \& Lunne, 2016)

\begin{tabular}{|l|l|l|l|l|}
\hline \multicolumn{1}{|c|}{ Method } & \multicolumn{1}{|c|}{ Technicalities } & \multicolumn{1}{c|}{ Source of errors } & \multicolumn{2}{c|}{ Practicalities } \\
\hline \multirow{3}{*}{ All the methods } & Tangent or "best-fit" lines definition & $\begin{array}{l}\text { Subjectivity } \\
\text { Scale of the plot (type) }\end{array}$ & $\begin{array}{l}\text { Easy if scale is } \\
\text { large }\end{array}$ & $\begin{array}{l}\text { Difficult if } \\
\text { scale is small }\end{array}$ \\
\cline { 2 - 5 } & Calculation / reading of $\mathrm{p}_{\mathrm{c}}{ }^{\prime}$ & Scale of the plot (size) & $\begin{array}{l}\text { Difficult if scale } \\
\text { is large }\end{array}$ & $\begin{array}{l}\text { Easy if scale } \\
\text { is small }\end{array}$ \\
\hline $\begin{array}{l}\text { Janbu } \\
\text { Becker } \\
\text { Karlsrud }\end{array}$ & $\begin{array}{l}\text { Additional calculations (i.e. incremental } \\
\text { work or deformation modulus) }\end{array}$ & Calculation mistakes & Extra time and effort required \\
\hline Casagrande & Definition of highest curvature point & Subjectivity & Difficult, it requires judgement \\
\hline Janbu & Undefined procedure & Subjectivity & $\begin{array}{l}\text { Difficult, it requires judgement } \\
\text { and experience }\end{array}$ \\
\hline
\end{tabular}

Umar and Sadrekarimi (2016) performed tests on resedimented samples of clay with plasticity indices ranging from 9 to 24. They found that the Boone method gave the closest approximation of preconsolidation pressure in reloading tests, with methods based on the slopes of the pre-yield and post-yield regions of the consolidation curve also performing very well. The Casagrande method was found to be inaccurate relative to these methods.

One of the results of the study by Umar and Sadrekarimi (2016) is that all the methods they investigated slightly overestimate the preconsolidation pressure. They mention that although the CRS test provides more points with which to construct the consolidation curve, it presents a new challenge in defining the pre-yield and post-yield lines due to the fact that these lines can be interpreted in multiple ways for the same test. They do not mention such a difficulty for the bilogarithmic method, which also depends on pre-yield and post-yield lines.

Table 2-4 summarizes which methods were included in each of the studies mentioned previously. 
Table 2-4: Summary of methods included in past comparative studies

\begin{tabular}{|l|l|}
\hline Author & Methods Studied \\
\hline Grozic et al., 2003 & Casagrande (1936) \\
& Janbu (1969) \\
& Burland (1990) \\
& Becker et al. (1987) \\
& Bilogarithmic \\
\hline Umar and Sadrekarimi, 2016 & Boone (2010) \\
& Oikawa (1987) \\
& Burland (1990) \\
& Onitsuka et al. (1995) \\
& Jose et al. (1989) \\
& Butterfield (1979) \\
& Pacheco Silva (1970) \\
& Casagrande (1936) \\
& Wang and Frost (2004) \\
& Jacobsen (1992) \\
\hline Paniagua et al., 2016 & Casagrande (1936) \\
& Janbu (1969) \\
& Pacheco Silva (1970) \\
& Becker et al. (1987) \\
& Karlsrud (1991) \\
\hline
\end{tabular}

\subsection{SUMMARY}

Past studies have all relied purely on graphical interpretation of consolidation test data. Many researchers have identified that the challenges associated with the graphical methods include locating the point of maximum curvature and interpreting effective stress visually, sometimes on a logarithmic scale.

The first challenge can be eliminated by developing an objective way of locating the point of maximum curvature. No reference to such a method has been found in the literature. Thus, the method introduced in this study must be evaluated.

The second challenge can be eliminated by using equations to plot the necessary straight lines and then calculating the intersection of these equations to determine the preconsolidation pressure. The only reference to the use of this approach found in the literature has been by Boone (2010), who only briefly mentions its advantages. This approach should be employed on a number of tests to see if it improves the accuracy of the graphical procedures.

Past studies comparing different methods to find preconsolidation pressure have not included the Sallfors method or the version of the Janbu method that uses the intersection of the straight line 
parts. These methods should thus be studied to determine their accuracy and their correlation with other methods. The bilogarithmic methods and the work method can both be classified as bilinear methods, as they define preconsolidation pressure as the intersection of pre-yield and post-yield lines. The $e$-logp bilinear method is also introduced, and the similarity of all these bilinear methods should be examined.

In addition, past studies have not included the Peck or Nagaraj et al. methods, as those studies were not concerned with sensitive clay. Although known preconsolidation pressures are not available against which to compare the results of the initial loading stages of the CRS tests, these methods should nonetheless be performed in order to compare the results of these two methods with those of other common methods.

Finally, there is no study available to apply different methods on sensitive Champlain Sea clay. Due to its unique compression properties, there are different challenges in identifying preconsolidation pressure for this soil type. It is worth the effort to identify the best method for Champlain Sea clay and help to evaluate the long-term settlement of Waba Dam. 


\section{CRS CONSOLIDATION TESTS}

\subsection{BACKgROUND ON CRS TEST}

Several types of consolidation test exist, including incremental loading, constant gradient, constant rate of loading and constant rate of strain. In the incremental loading test, the sample is subjected to a constant load, and the deformation of the sample due to this applied load is tracked over time. After some amount of time (typically 24 hours, although variations on the test exist), a subsequent load is added and the process is repeated. The loading increment ratio refers to the relationship between a given load and the next applied load. The final deformation of the sample (which can also be expressed as void ratio) for each stress increment is then plotted against the stress that was applied. Thus, the number of points that define the e-logp curve is equal to the number of stress increments applied.

The incremental load test with loading increment ratio of 1 has been found to be unsuitable for soft, plastic clays (Silvestri, Yong, Soulié, \& Gabriel, 1986). This is in part due to the collapsing nature of the clay, causing the preconsolidation pressure to be underestimated by this testing type. In addition, due to the small number of data points obtained in such a test, the user has a great amount of freedom in drawing the curve, which might not capture the true behaviour of the soil. Modifications to the standard oedometer test have been proposed. In Canada, it is common for a loading increment ratio of 0.5 rather than 1 to be used (Silvestri, Yong, Soulié, \& Gabriel, 1986). However, this greatly increases the amount of time required to perform the test.

The CRS test does not have this limitation, as hundreds or potentially thousands of points are available with which to draw the $e$-logp curve. The CRS test is also much faster to execute than the incremental load test, which can take 10 days even when the standard loading increment ratio is used.

\subsection{THEORY OF CRS CALCULATIONS}

An approximate linear solution for constant rate of strain testing was proposed by Smith and Wahls in 1969, and a thorough linear solution accounting for transient effects was published by Wissa et al. in 1971. The test is said to be in a transient condition when the strain distribution in the specimen experiences a time-dependent variation at the beginning of a loading or unloading stage or when the strain rate changes. Once this variation dissipates and the average value of strain distribution is time-independent, the test is said to be in a steady state (ASTM, 2012).

Currently, the linear theory is the ASTM standard for CRS testing, although the non-linear theory is also considered acceptable by ASTM. The assumption of linear theory is that the soil has a constant coefficient of volume compressibility $\left(\mathrm{m}_{\mathrm{v}}\right)$. This means that the relationship 
between strain and stress is linear. Non-linear theory assumes that the soil has a constant compression index $\left(\mathrm{C}_{\mathrm{c}}\right)$, meaning that the relationship between strain and logarithm of stress is linear.

The following description of the CRS theory is adapted from Adams (2011). The linear theory proposed by Smith and Wahls applies only to steady state conditions and is based on Terzaghi's basic theory of consolidation. The basic equation of consolidation is:

$$
\frac{\delta}{\delta z}\left(\frac{k}{\gamma_{w}} \frac{\delta u}{\delta z}\right)=\frac{1}{1+e} \frac{\delta e}{\delta t}
$$

By assuming that the hydraulic conductivity is a function of void ratio, it thus becomes a function only of time. Thus, the hydraulic conductivity is independent of the vertical position in the control volume. This allows the small strain assumption to be made, which is that the change in void ratio with time is small. Because the strain rate is constant and the specimen is laterally confined, the change in volume is also constant. Thus, the change in void ratio, $\frac{\delta e}{\delta t}$, is also constant.

By making these assumptions, the excess pore pressure at the base of the specimen is a function of the relationship between the hydraulic conductivity and the void ratio. This is:

$$
\Delta u_{b}=\frac{\gamma_{w} s H^{2}}{k\left(1-e_{\text {avg }}\right)}\left(\frac{1}{2}-\frac{b}{12 r}\right)
$$

In the above equation, $\mathrm{e}_{\text {avg }}$ is the average void ratio at time $\mathrm{t}, \mathrm{H}$ is the height of the specimen at time t. The parameter $b$ a constant depending on the soil's material properties, and $r$ is given by:

$$
r=-\frac{\delta e_{\text {avg }}}{\delta t}=-\frac{1}{V_{s}} \dot{\epsilon} A
$$

where $\dot{\epsilon}$ is the strain rate and $\mathrm{A}$ is the sample cross-sectional area.

When the $\mathrm{r}$ and $\mathrm{b}$ parameters are known, the above formula can be used to calculate the hydraulic conductivity directly, from which the coefficient of consolidation can be calculated as:

$$
c_{v}=\frac{k}{m_{v} \gamma_{w}}
$$


However, as the ratio b/r must be either known or assumed, this is considered only a partial solution. This problem is resolved in the solution presented by Wissa et al. in 1971, as follows.

Based on additional assumptions, the basic equation of consolidation can be written in terms of strain as:

$$
c_{v} \frac{\delta^{2} \epsilon}{\delta z^{2}}=\frac{\delta \epsilon}{\delta t}
$$

The above equation has the solution:

$$
\epsilon\left(X, T_{v}\right)=\dot{\epsilon} t\left[1+F\left(X, T_{v}\right)\right]
$$

where:

$$
\begin{gathered}
X=\frac{Z}{H} \\
T_{v}=\frac{c_{v} t}{H^{2}} \\
F\left(X, T_{v}\right)=\frac{1}{6 T_{v}}\left(2-6 X+3 X^{2}\right)-\frac{2}{\pi^{2} T_{v}} \sum_{n=1}^{\infty}\left[\frac{\cos (n \pi x)}{n^{2}} e^{-n^{2} \pi^{2} T_{v}}\right]
\end{gathered}
$$

\subsection{Equations Used for Data Processing}

The formulas used for processing the data provided by the CRS machine are based on ASTM D4186 (ASTM, 2012). These formulas are based the theory described above.

Before data processing can begin, it is necessary to first convert the machine outputs to useful engineering values. This is done according to the user's manual provided by the manufacturer of the CRS machine (Trautwein, 2001).

$$
P=\frac{C F *\left(V_{s}-V_{0}\right)}{V_{e}}
$$

where: $\mathrm{P}=$ physical input (e.g. load, displacement) (unit of the physical input)

$$
\mathrm{CF}=\text { calibration factor (unit/volt/volt) }
$$




$$
\begin{aligned}
& \mathrm{V}_{\mathrm{s}}=\text { sensor output voltage (volts) } \\
& \mathrm{V}_{0}=\text { sensor voltage when zero stress is applied (volts) } \\
& \mathrm{V}_{\mathrm{e}}=\text { sensor excitation voltage (volts) }
\end{aligned}
$$

The outputs provided by the machine are: Load (lbs); Displacement (in); Pore Pressure (psi); Cell Pressure (psi); and Pump Pressure (psi). Since the machine is set to imperial units, the appropriate unit conversions are then applied so that the values will be in metric units.

Axial stress $(\mathrm{kPa})$ at any given time step is calculated as:

$$
\sigma_{a, i}=\frac{P_{i}}{A} * 1000
$$

where: $\quad \mathrm{P}=\operatorname{load}(\mathrm{N})$

$$
A=\text { cross-sectional area of the sample with diameter of } 63.5 \mathrm{~mm}\left(\mathrm{~mm}^{2}\right)
$$

Axial strain (\%) at any given time step is calculated as:

$$
\epsilon_{i}=\frac{H_{i}-H_{0}}{H_{0}} * 100
$$

where: $\quad \mathrm{H}_{\mathrm{i}}=$ initial height of sample (mm)

$$
\mathrm{H}_{0}=\text { current height of sample (mm) }
$$

Void ratio at any given time step is calculated as:

$$
e_{i}=e_{0}-\left(\frac{\epsilon_{i}}{100}\right)\left(1+e_{0}\right)
$$

where: $\quad \mathrm{e}_{0}=$ initial void ratio

The recommendation of ASTM D4186 is to calculate excess pore pressure as the difference between current pore pressure and current cell pressure. However, based on the assumptions of that cell pressure is constant and equal to the initial base pore pressure, the excess pore pressure $(\mathrm{kPa})$ at any given time step is calculated as:

$$
\Delta u_{i}=u_{i}-u_{0}
$$


where: $\quad \mathrm{u}_{\mathrm{i}}=$ current base pore pressure $(\mathrm{kPa})$

$$
\mathrm{u}_{0}=\text { initial base pore pressure }(\mathrm{kPa})
$$

Strain rate (/s)at any given time step is calculated as:

$$
\dot{\varepsilon}_{i}=\frac{\left(\epsilon_{i+1}-\epsilon_{i-1}\right) / 100}{t_{i+1}-t_{i-1}}
$$

where: $\quad \mathrm{t}=$ time (seconds)

Steady state factor is a unitless parameter that is calculated in order to determine whether the transient effects are sufficiently small for the test to be considered valid. The transient effects are considered to be insignificant when $\mathrm{F}>0.4$. This parameter is calculated as:

$$
F_{i}=\frac{\left(\sigma_{a, i}-\sigma_{a, 0}\right)-\left(\Delta u_{i}-\Delta u_{0}\right)}{\sigma_{a, i}-\sigma_{a, 0}}
$$

Effective stress $(\mathrm{kPa})$ at any given time step is calculated as:

$$
\sigma_{i}^{\prime}=\sigma_{a, i}-\frac{2}{3} \Delta u_{i}
$$

Hydraulic conductivity $(\mathrm{m} / \mathrm{s})$ at any given time step is calculated as:

$$
k_{i}=\frac{\dot{\varepsilon}_{i} H_{i} H_{0} \gamma_{w}}{2 \Delta u_{i}} * \frac{1}{10000}
$$

where: $\quad \gamma_{\mathrm{w}}=9.81 \mathrm{kN} / \mathrm{m}^{3}$

Coefficient of volume compressibility $\left(\mathrm{m}^{2} / \mathrm{kN}\right)$ at any given time step is calculated as:

$$
m_{v, i}=\frac{\epsilon_{i+1}-\epsilon_{i-1}}{\sigma_{i+1}^{\prime}-\sigma_{i-1}^{\prime}} * \frac{1}{100}
$$

Coefficient of consolidation $\left(\mathrm{m}^{2} / \mathrm{s}\right)$ at any given time step is calculated as: 


$$
c_{v, i}=\frac{k_{i}}{m_{v, i} \gamma_{w}}
$$

Finally, pore pressure ratio (unitless) at any given time step is calculated as:

$$
R_{i}=\frac{\Delta u_{i}}{\sigma_{a, i}}
$$

\subsection{Experimental Procedures}

The procedure used to prepare samples and perform the tests was established based the recommendations and practices of past researchers and based on the equipment available at the Ryerson University geotechnical laboratory.

The CRS machine used in the testing program was manufactured by Trautweing Soil Testing Equipment Company. The figure below shows the consolidation cell with all connections of sensors and tubes.

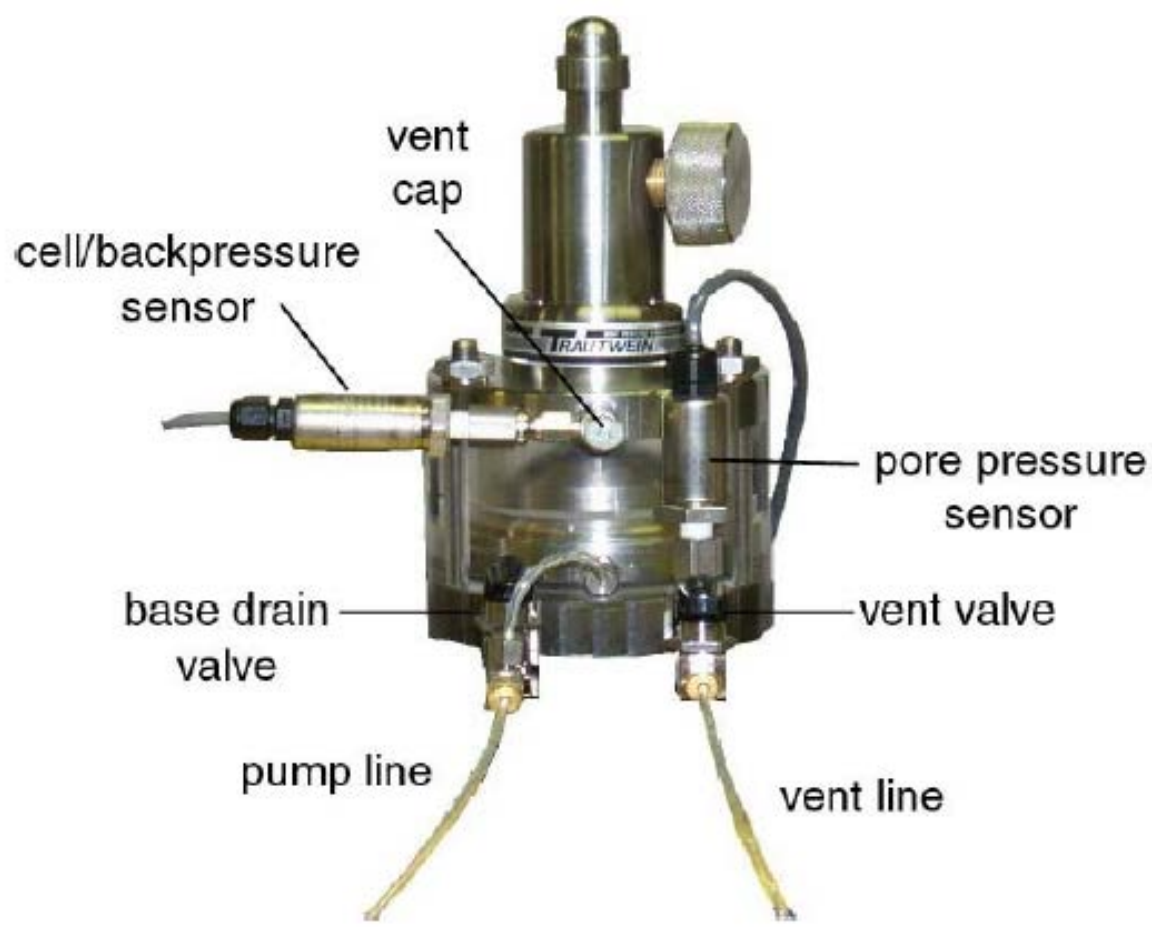

Figure 3-1: Connection of consolidation cell (Trautwein, 2001) 
The procedure to prepare the consolidation sample, set up the cell, start the test, and disassemble the cell is as follows:

1. Saturate the top and bottom porous stone by immersing them in distilled water and subjecting them to sonic waves

2. Remove a block of soil from the Laval sample with top and bottom leveled off

3. Apply grease to the inside of a cutter ring and measure its weight

4. Trim samples carefully into the ring using the trim and taper method

5. Smooth the top and bottom surfaces first using a wire saw, then using a sharp straightedge

6. If necessary, fill gaps with remoulded trimmings

7. After sample preparation is finished, determine the combined mass of the soil and ring

8. Measure the height of soil in the ring using a caliper

9. Determine the combined mass of the soil, ring, top porous stone and one dry filter paper

10. Place the filter paper and top porous stone on top of the sample and determine their combined height

11. Place the bottom porous stone inside the cavity of the base

12. Place the sample on the base

13. Cover the rubber gasket with grease and place it around the cutter ring

14. Apply grease on the bottom of the cell wall to prevent leaks

15. Ensure that the piston is in a raised position, then attach the cell wall to the base using the appropriate bolts

16. Unlock the piston and allow it to come into contact with the top porous stone. Then, lock the piston once it is in a lowered position

17. Insert the pore pressure sensor

18. Connect the pump to the base, and then flood the base. Once water is seen to exit the base through the pore pressure sensor, ensure that the sensor is flushed. Then, turn off the pump and close both the pore pressure sensor valve and the valve connecting the pump to the base

19. Insert the cell pressure sensor at the top of the cell, and then fill the cell. When water starts to exit through the sensor, ensure that the sensor is flushed, then stop the pump and cap the sensor

20. Select and apply a target cell pressure and ramp time, along with a target seating strain

21. Allow the loading frame to come into contact with the piston

22. When prompted, unlock the piston and check that strain gage is functioning properly

23. Allow the machine to start applying the seating strain, then leave the sample to saturate overnight

24. The next day, check that the proper loading schedule has been specified in the program, then start the loading and unloading stages 
25. After the test has been completed, save the raw data to an external drive

26. Release the cell pressure and empty the cell

27. Remove the cell wall from the base

28. Remove the cutter ring from the base

29. Determine the combined height and combined mass of the ring, soil, top porous stone and filter paper after the test

30. Extrude the soil into a ceramic bowl with known mass to obtain the water content of the soil after the test as well as the dry mass of the soil

The images below show excerpts from the sample preparation process.

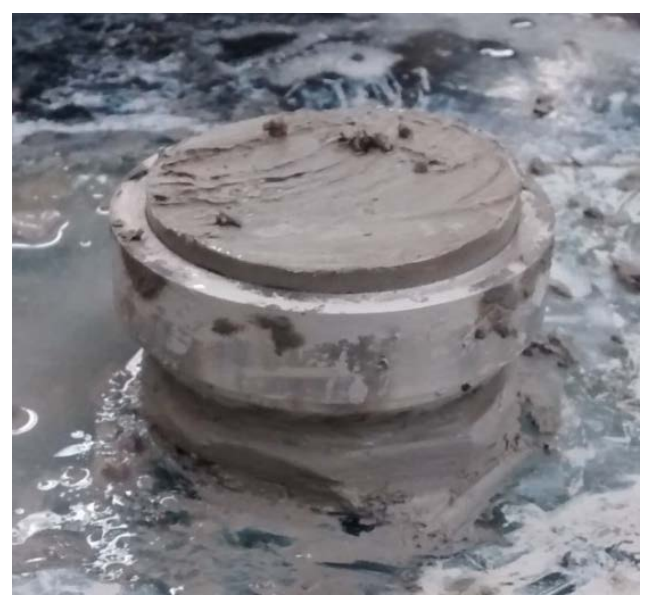

a) Before removing excess soil.

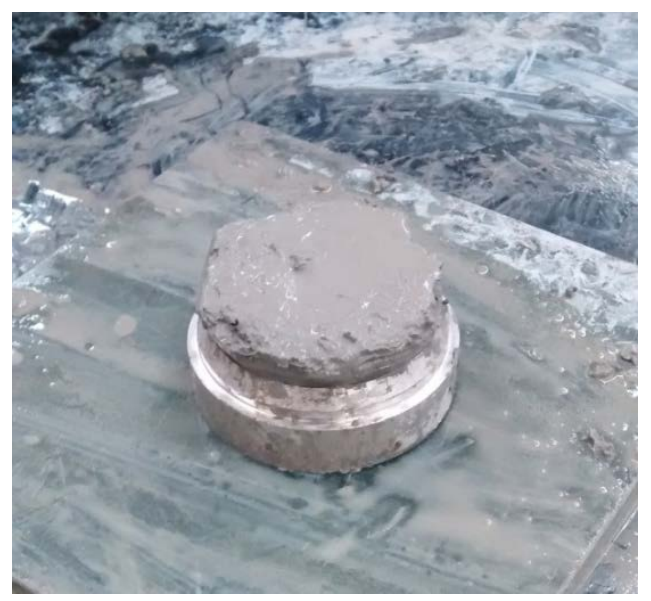

b) After removing bottom excess soil 


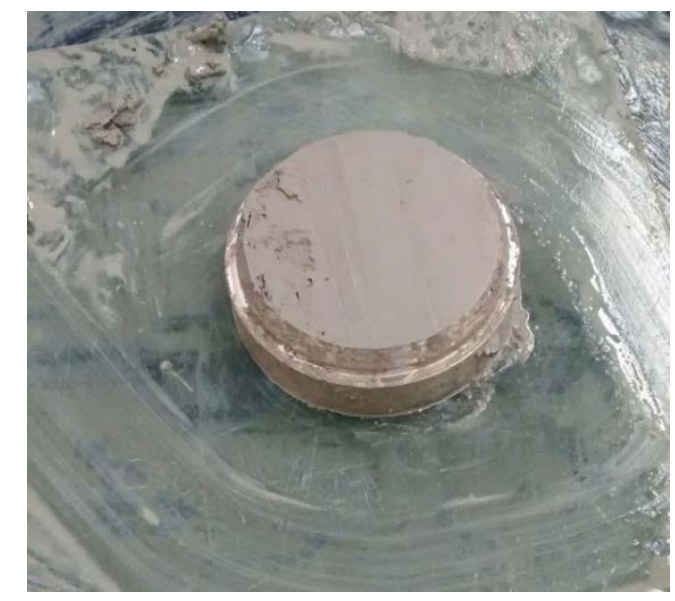

c) After removing top and bottom excess soil

Figure 3-2: Sample trimming process

\subsection{Typical Plots Available from CRS Test Data}

Due to large number of parameters that are calculated from CRS test data, a number of plots can be produced from a single test to provide insight into a soil's behaviour under loading. A selection of such plots is provided in this section. All the plots shown in this section correspond to the test performed on a sample from a depth $11.36 \mathrm{~m}$ below the top of the stabilizing berm, performed using a strain rate of $1 \%$ /hour. Only a small number of these plots are utilized in the current study.

The most obvious plot that can be produced from CRS data is a void ratio vs. effective stress $(e$ $\log$ ) curve. The logarithmic scale of the horizontal axis is typically represented by writing 1,10 , 100,1000 , etc. on equally spaced gridlines. An equally valid way to represent the logarithmic scale is to write the coefficients to which ten is raised. Thus, the numbers 1, 10, 100 and 1000 would be represented by $0,1,2$, and 3 , respectively. When represented in this way, the horizontal axis is linear.

An example of an e-logp curve is shown in Figure 3-3. The sizes of the scales are intentionally selected such that the length of one log cycle is equal to the length of an increment of 1 on the void ratio axis. 


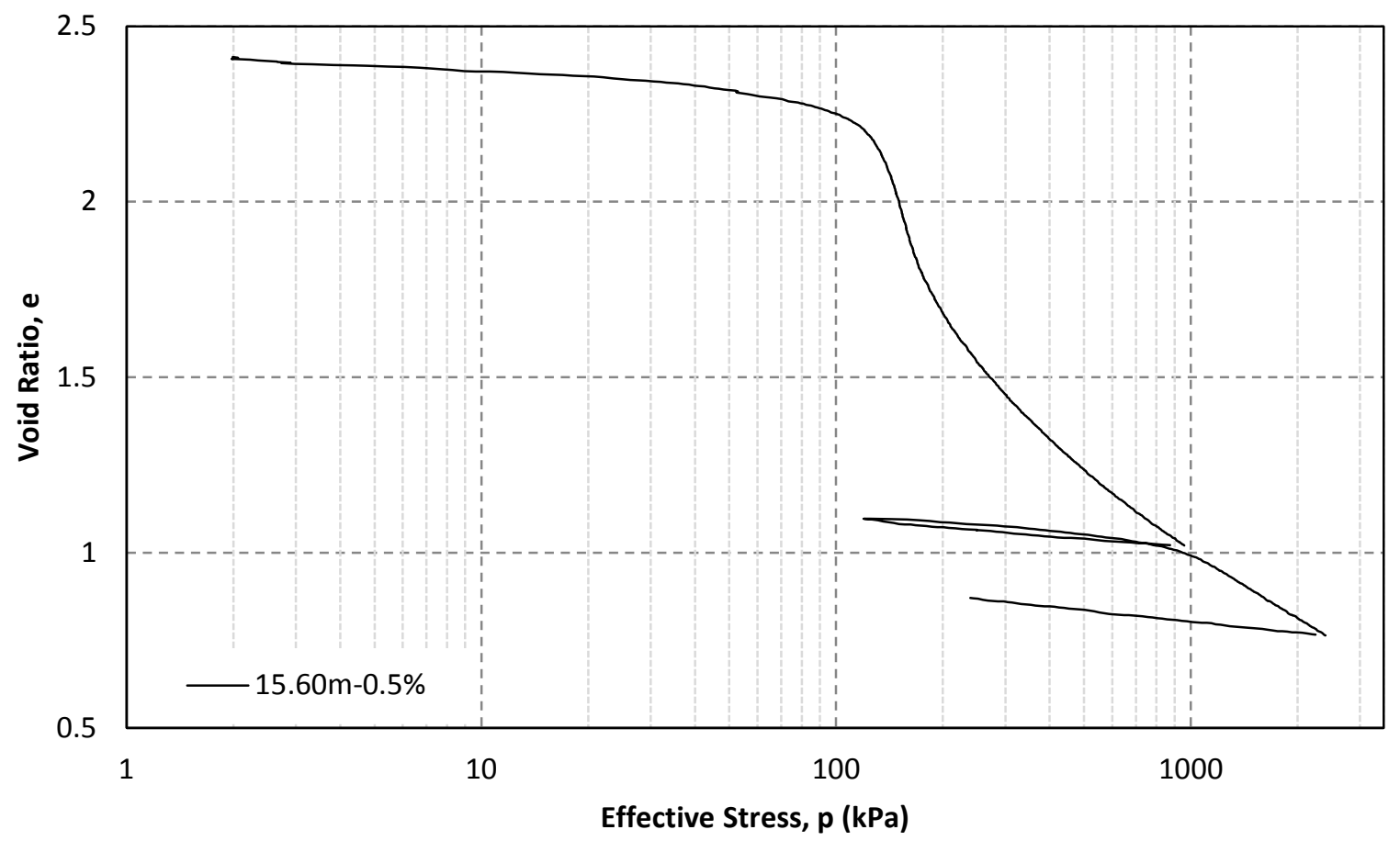

Figure 3-3: e-logp curve (AKA consolidation curve)

Void ratio can also be plotted against hydraulic conductivity on a logarithmic scale. After a stable condition is reached, this results in a straight line, indicating that as a soil consolidates and the pores become smaller, there is a corresponding decrease in conductivity. The slope of this line is known as $c_{k}$. If the data is trimmed to remove the points that occur before steady state is reached, then regression can be used to calculate the slope of this line. The figure below shows such a plot along with the logarithmic regression equation. The hydraulic conductivity values calculated for the unloading stages are not included in these plots. 


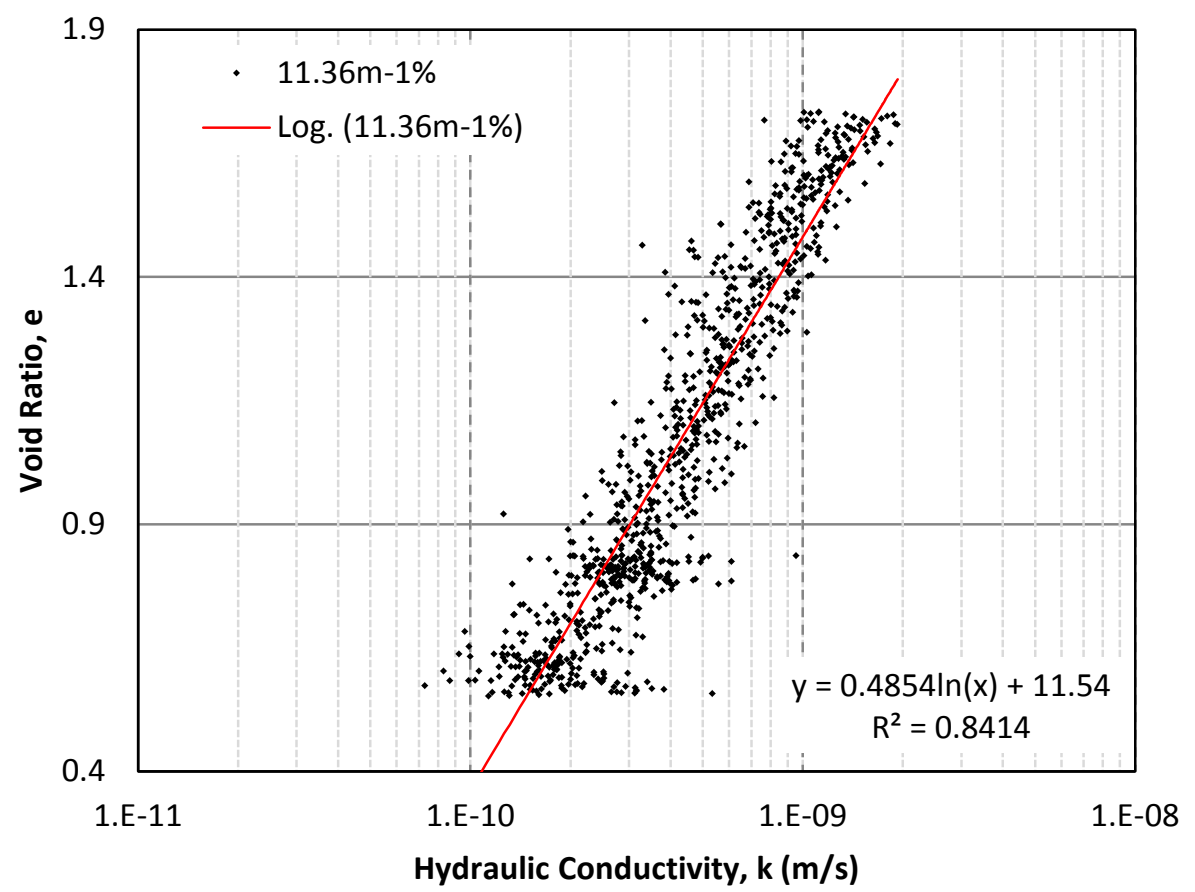

Figure 3-4: e-logk curve with data trimmed for steady state showing natural logarithmic regression equation 
Another way to view the hydraulic conductivity data is to plot it against effective stress, with both parameters plotted on a logarithmic scale. This is shown in Figure 3-5. The hydraulic conductivity values calculated for the unloading stages are once again excluded from this plot. As with the remainder of the following plots, the effective stress axis is shown to start at $10 \mathrm{kPa}$ to remove the influence of seating errors on the appearance of the plots. Once again, a linear trend can be observed. The line representing this trend in Figure 3-5 was not created by regression, and is included for demonstrative purposes. It can be noted that neither the $e$-logk nor the logk-logp plots show a break that could correspond to preconsolidation pressure.

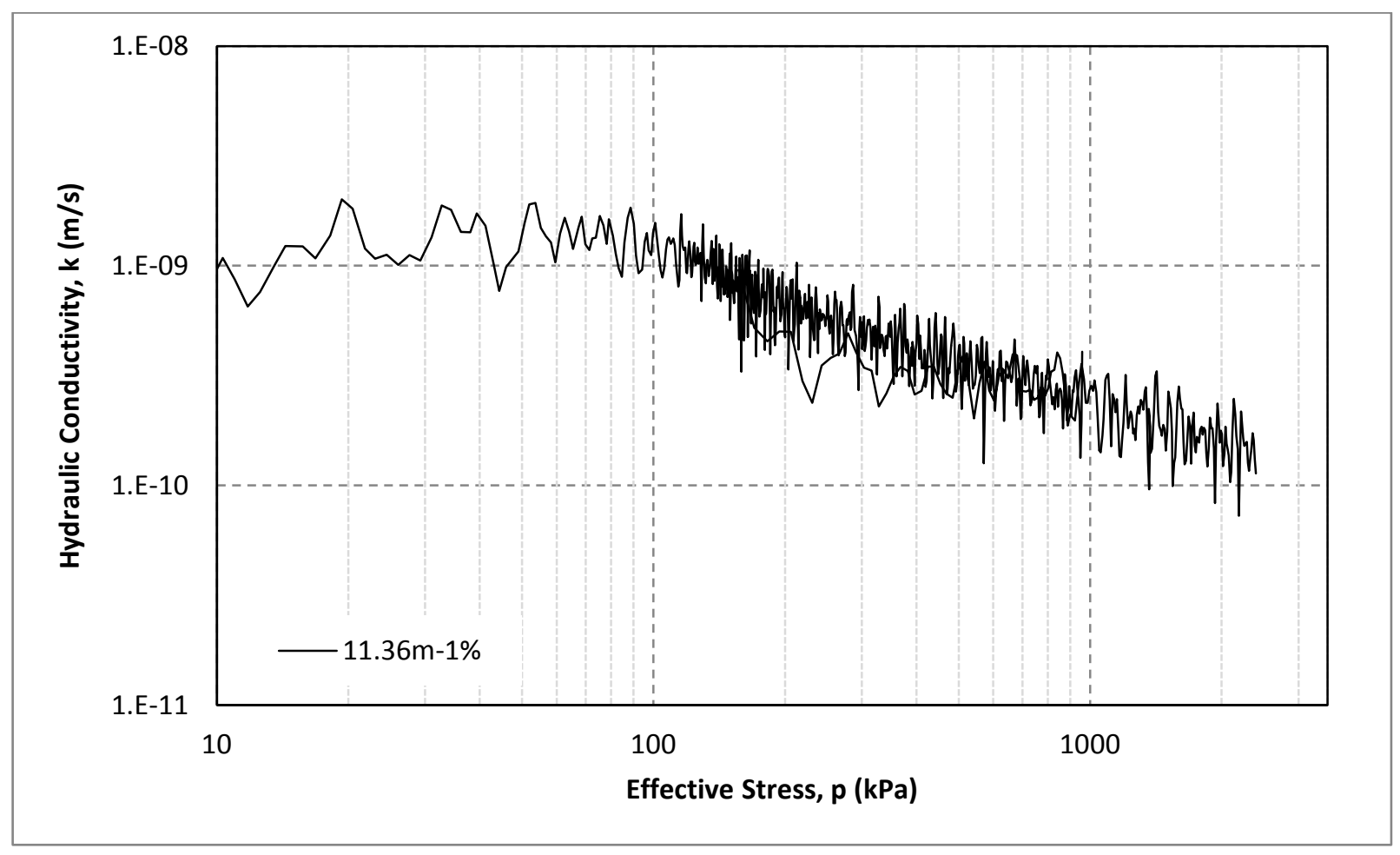

Figure 3-5: Hydraulic conductivity plotted against effective stress

The coefficient of volume compressibility can also be plotted against effective stress, with Figure 3-6 showing such a plot. Once again, the unloading stages are included, showing $\mathrm{m}_{\mathrm{v}}$ values that are lower than those obtained in the loading stages. The reloading part of the $\log _{v}$-logp resumes the path it had previously been following. It can also be noted that the plot shows a peak, after which it drops. The lines shown in the plot below were not obtained by regression and are included for demonstrative purposes. 


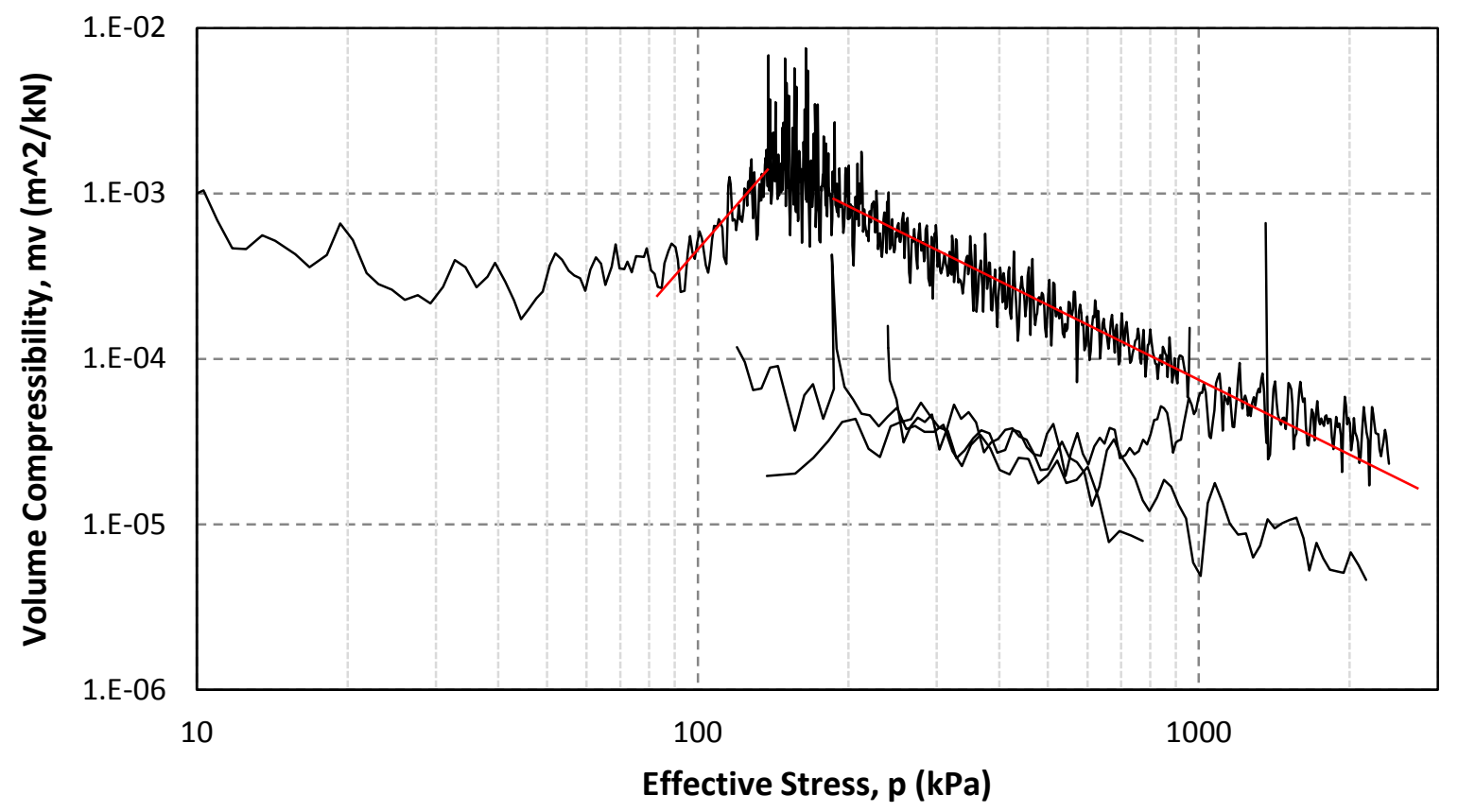

Figure 3-6: Coefficient of volume compressibility plotted against effective stress

When the coefficient of consolidation is plotted against effective stress, a plot such as Figure 3-7 is obtained. The coefficient of consolidation is related to both hydraulic conductivity and coefficient of volume compressibility, which are shown in the preceding plots. It is thus not surprising that the plot of coefficient of consolidation vs. effective stress appears related to those other plots. Here, a minimum value is obtained, due to the fact that $\mathrm{C}_{\mathrm{v}}$ is inversely proportional to $\mathrm{m}_{\mathrm{v}}$. The path of the $\log _{c_{v}} \log p$ plot appears more curved than the previous plots. Once again, the unloading stages are included, and curve in the reloading stage eventually resumes the path it had been following prior to unloading.

The pore pressure ratio, which is defined as the ratio between base excess pressure and total axial stress, yields a plot as shown in Figure 3-8 when plotted against effective stress. It should be noted that pore pressure ratio is plotted on a linear scale. It can be noticed that, in the early part of the test, very high values of pore pressure ratio are observed, but they then drop to below the maximum tolerable value for a CRS test to be considered valid. The high initial values are the result of seating error. Once again, the values for the unloading stages are shown in the above plot. During the unloading stages, the pore pressure ratio has a negative value, due to the negative pore pressures that are developed. After reloading commences, the pore pressure ratio climbs, reaches a peak, and then decreases. 


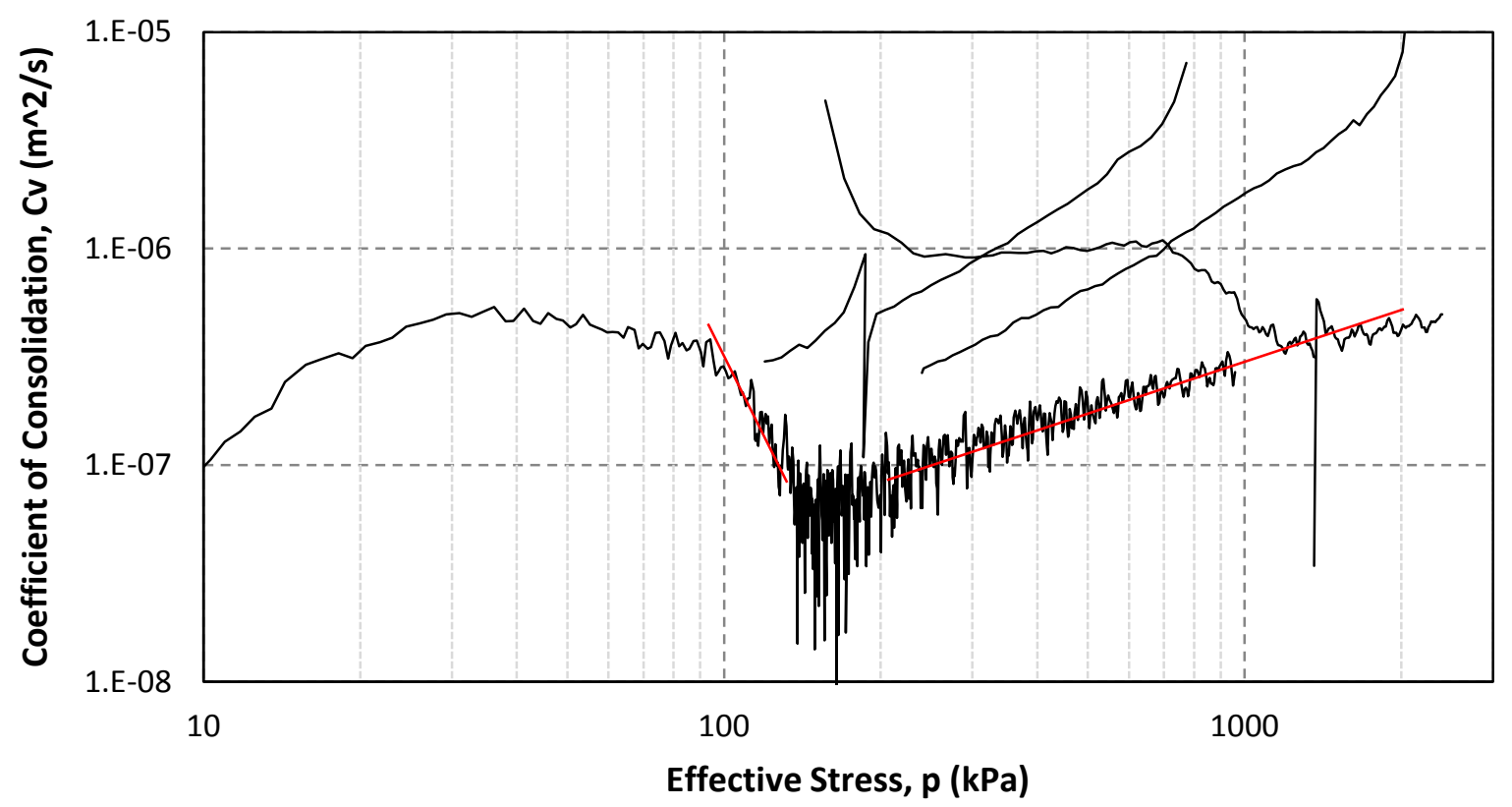

Figure 3-7: Coefficient of consolidation plotted against effective stress

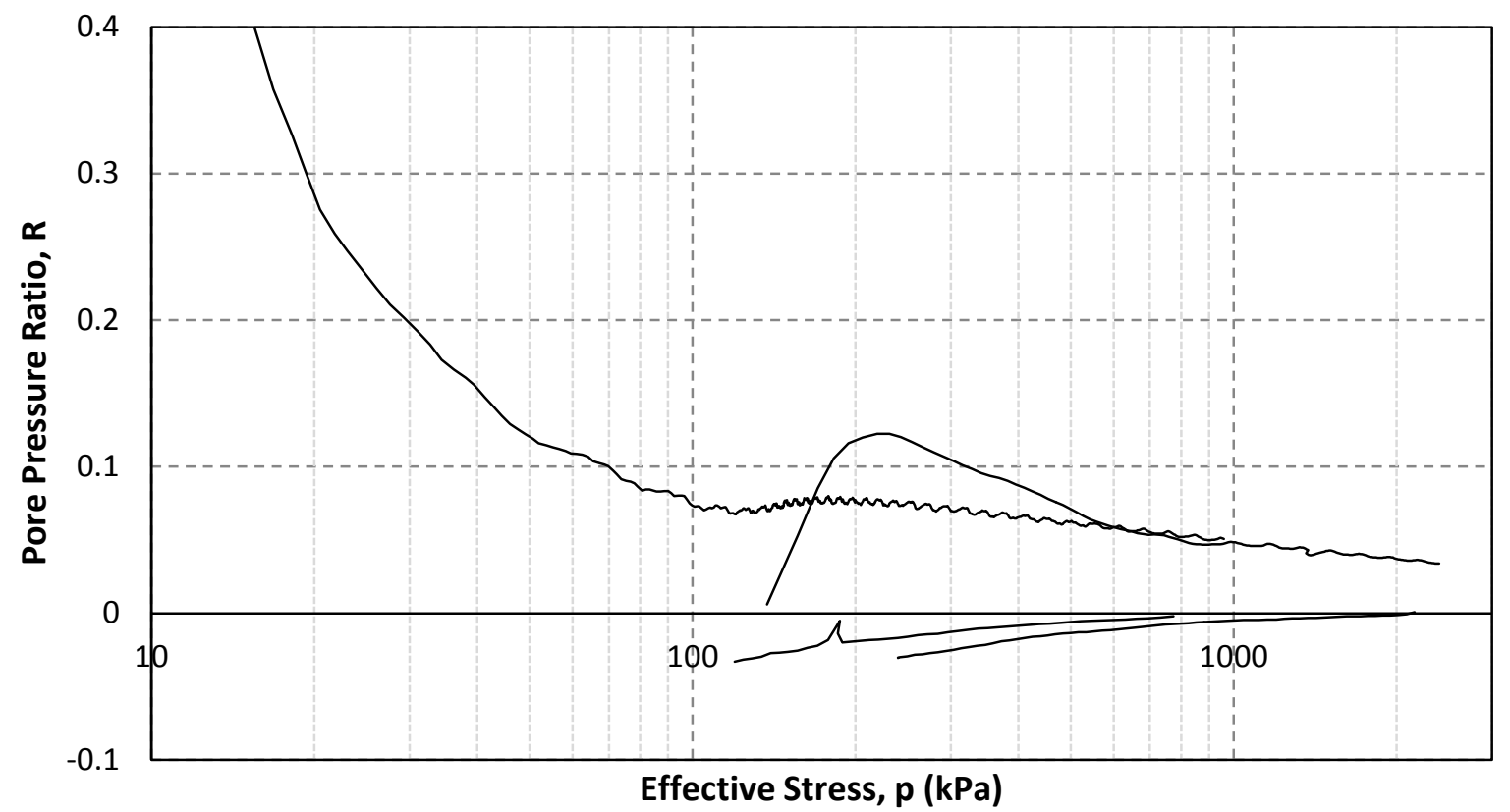

Figure 3-8: Pore pressure ratio plotted against effective stress 


\subsection{Experimental Results on Champlain Sea Clay}

All tests performed for this study were done on samples from the Upper Clay layer from the Waba Dam site.

Table 3-1 lists each test with the corresponding loading strain rate. The third column indicates whether the sample was trimmed in such a way that the direction of loading during the CRS test is transverse to the ground surface in the field. These samples are denoted as vertically oriented. For simplicity, samples that are horizontally oriented are not given a label in this column. The graphical methods to determine preconsolidation pressure was performed on the initial loading stage for all tests. However, for two of the tests, the stress at unloading was too low to obtain a useful reloading curve. These tests are indicated with "No" in the right column.

Table 3-1: Summary of CRS tests performed

\begin{tabular}{|c|c|c|c|}
\hline \multirow{2}{*}{ Depth } & $\begin{array}{c}\text { Loading Strain } \\
\text { Rate (\%/hour) }\end{array}$ & $\begin{array}{c}\text { Vertically } \\
\text { Oriented? }\end{array}$ & $\begin{array}{c}\text { Used for } \\
\text { Reloading } \\
\text { p'c? }\end{array}$ \\
\hline \multirow{2}{*}{$11.36 \mathrm{~m}$} & 0.5 & & \\
& 1 & & \\
\hline \multirow{3}{*}{$15.60 \mathrm{~m}$} & 0.5 & & \\
& 1 & & \\
\cline { 2 - 4 } & 5 & & \\
\cline { 2 - 4 } & 1 & Yes & \\
\hline $30.48 \mathrm{~m}$ & 1 & & \\
\cline { 2 - 4 } & 2 & & No \\
\hline $34.90 \mathrm{~m}$ & 1 & Yes & No \\
\hline $39.48 \mathrm{~m}$ & 1 & & Yes \\
\cline { 2 - 4 } & 1 & & \\
\hline
\end{tabular}

In the following sections, the $e$-log plots for each test will be presented. The data for each test has been trimmed to remove the effect of seating error. The test labels contain the sample depth and the loading strain rate. Tests performed on vertically oriented sample have the suffix "kx". For example, the test performed at the depth of $15.60 \mathrm{~m}$ with a strain rate of $1 \%$ /hour in a vertically oriented trimming direction is labelled " $15.60 \mathrm{~m}-1 \% \_\mathrm{kx}$ ".

For consistency, the horizontal scales on all plots have the same range. Where convenient, the vertical scales also have the same range. Furthermore, the dimensions of the plots are such that the inner boxes appear as squares. This is equivalent to drawing one log cycle on the horizontal axis the same length as an increment of 1 on the vertical axis. This reflects the scale ratio of e: $\log p=1: 1$ that is assumed when determining the point of maximum curvature. 
The horizontal axis in the following plots has a linear scale representing the exponent on 10 required to produce a logarithmic scale. Thus, the coordinate that corresponds to $100 \mathrm{kPa}$ appears as 2, $1000 \mathrm{kPa}$ appears as 3, and so on. It should be noted that marking stress in this way has no influence on the shape of the e-logp plot.

\subsubsection{Samples from $11.36 \mathrm{~m}$}

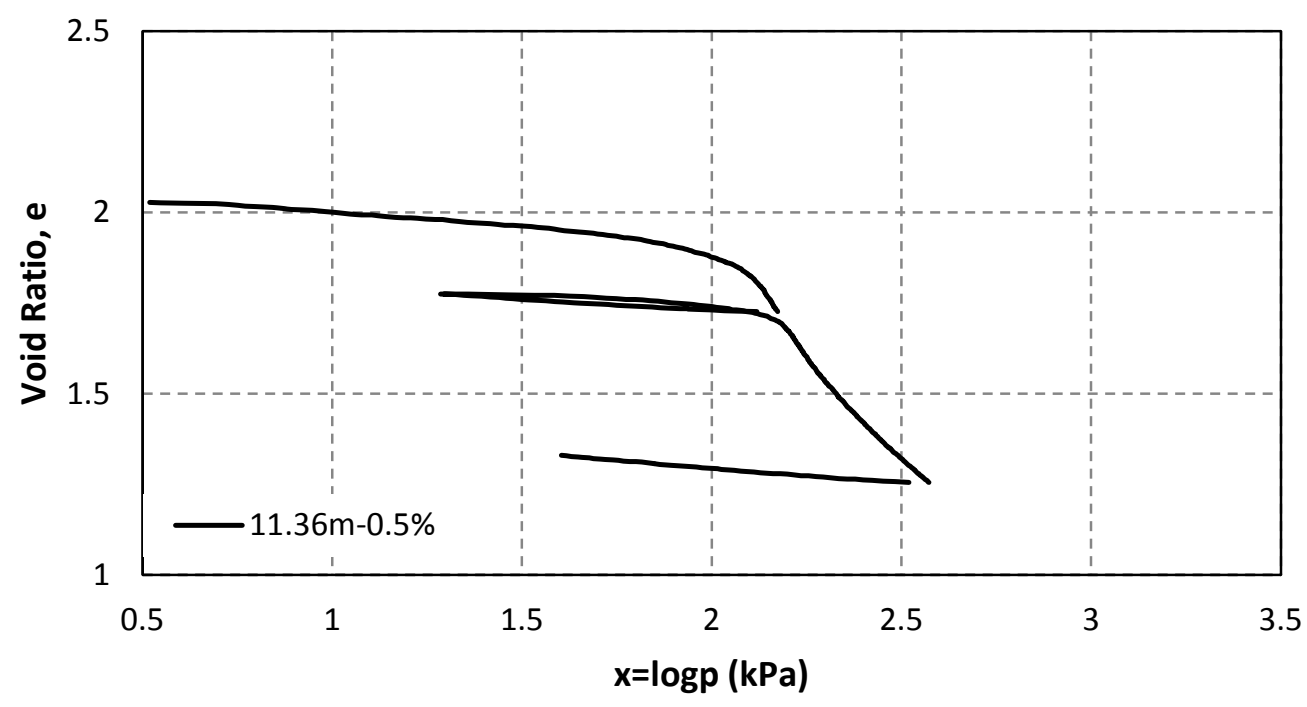

Figure 3-9: e-logp plot from 11.36-0.5\% test

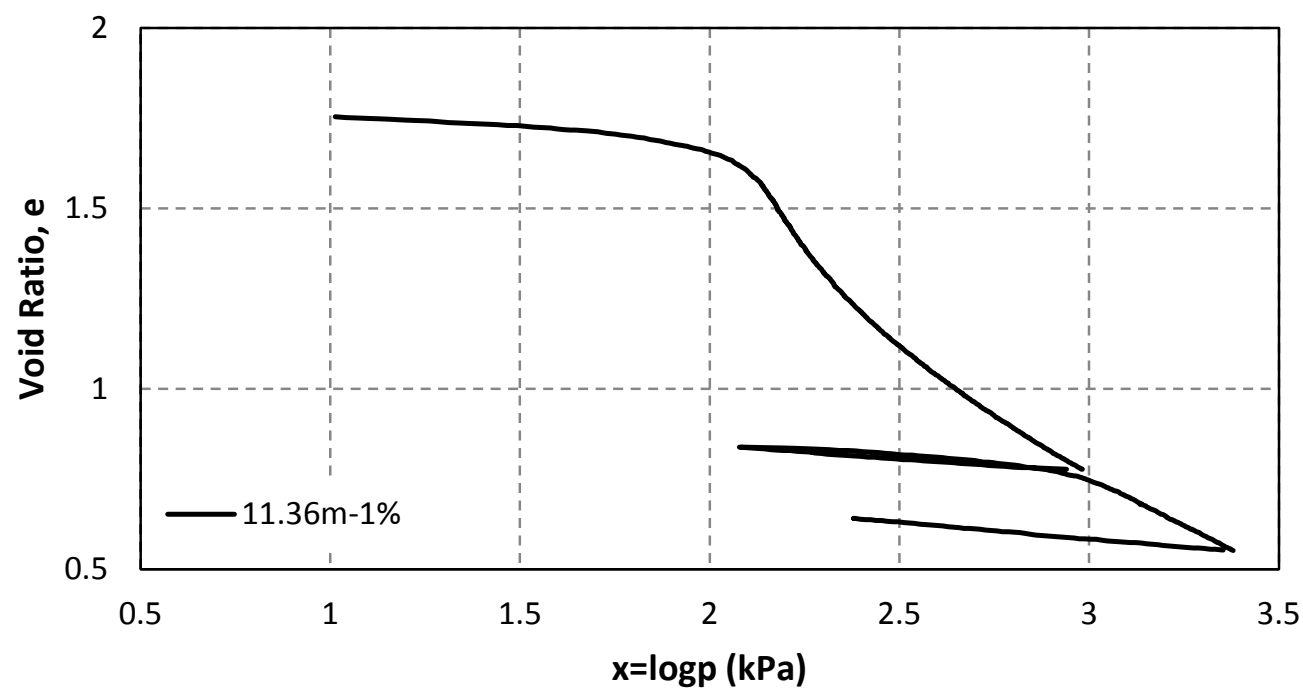

Figure 3-10: e-logp plot from 11.36m-1\% test 


\subsubsection{Samples from $15.60 \mathrm{~m}$}

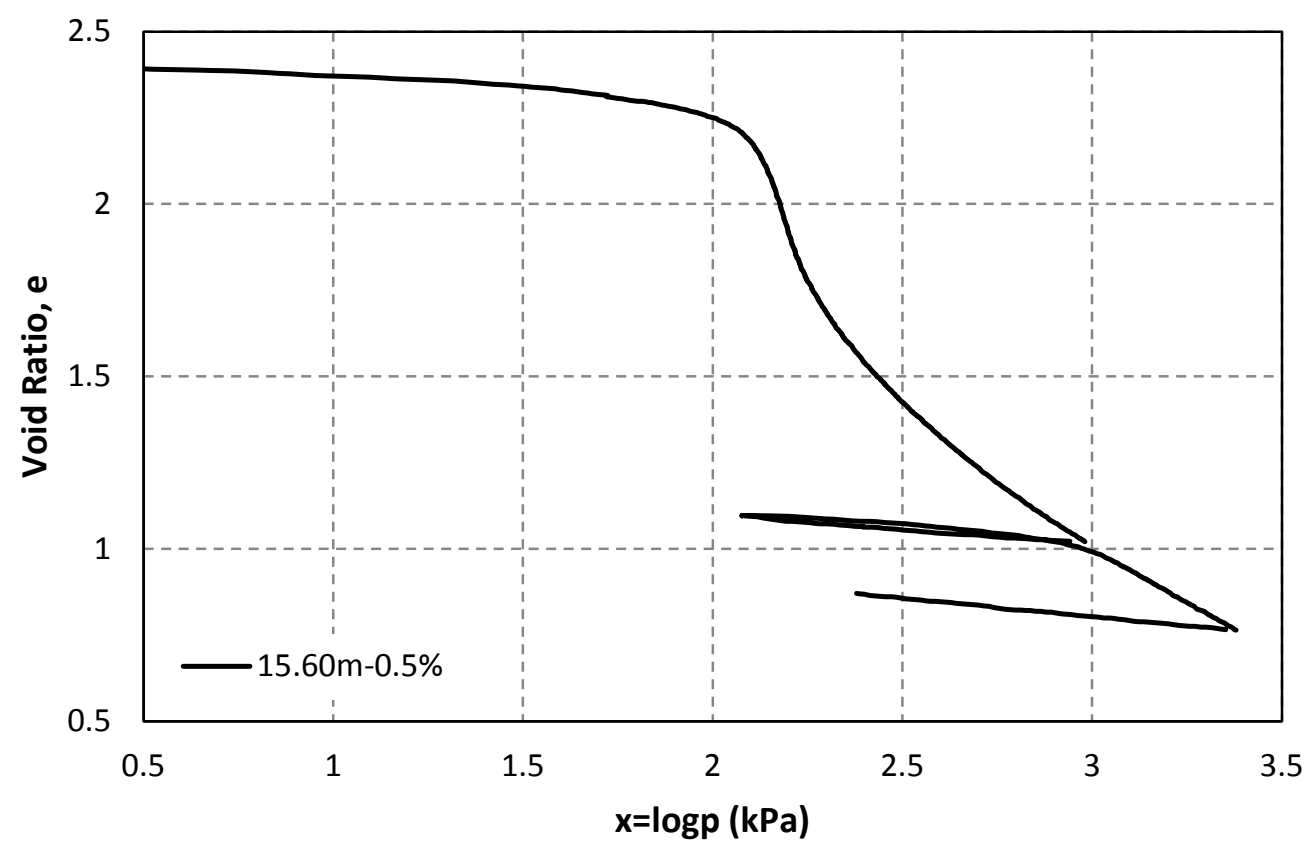

Figure 3-11: e-logp plot from $15.60 \mathrm{~m}-0.5 \%$ test

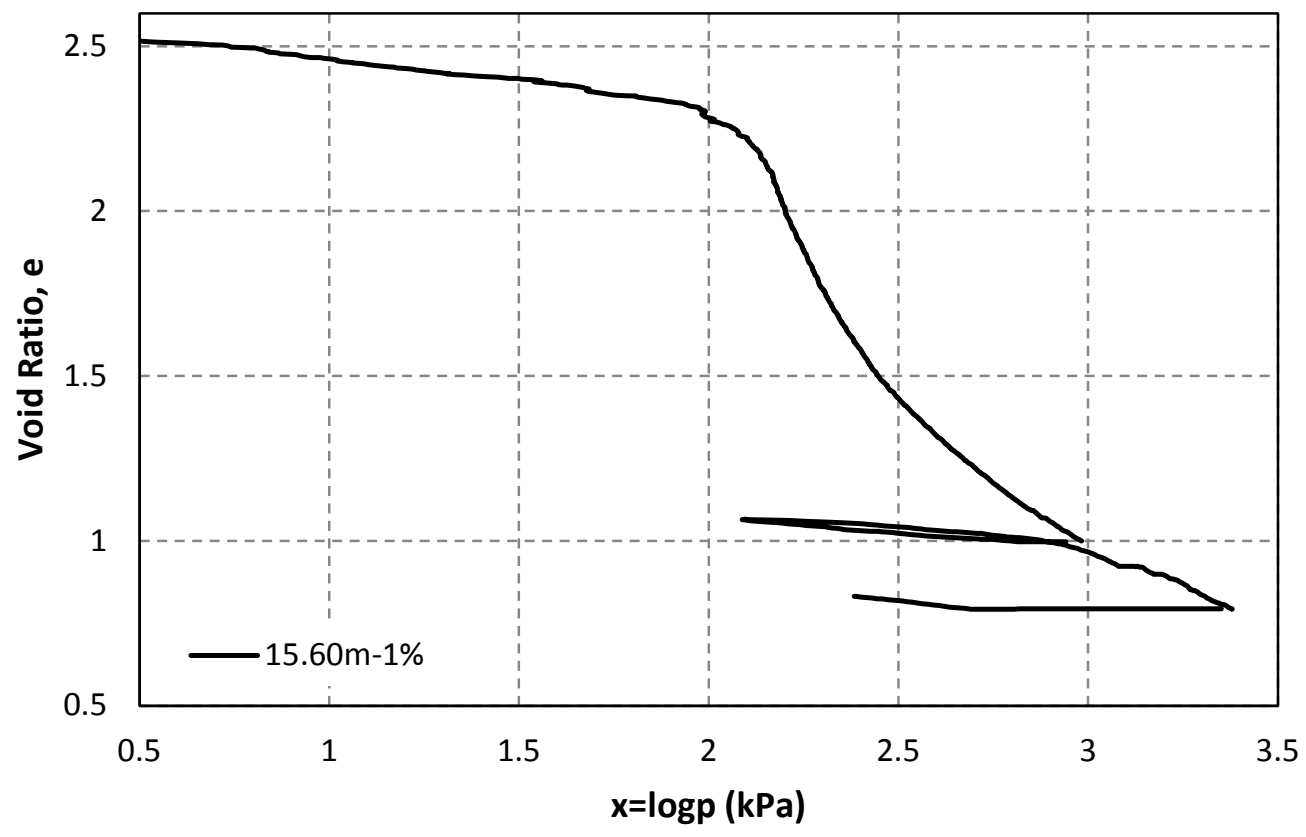

Figure 3-12: e-logp plot from $15.60 \mathrm{~m}-1 \%$ test 


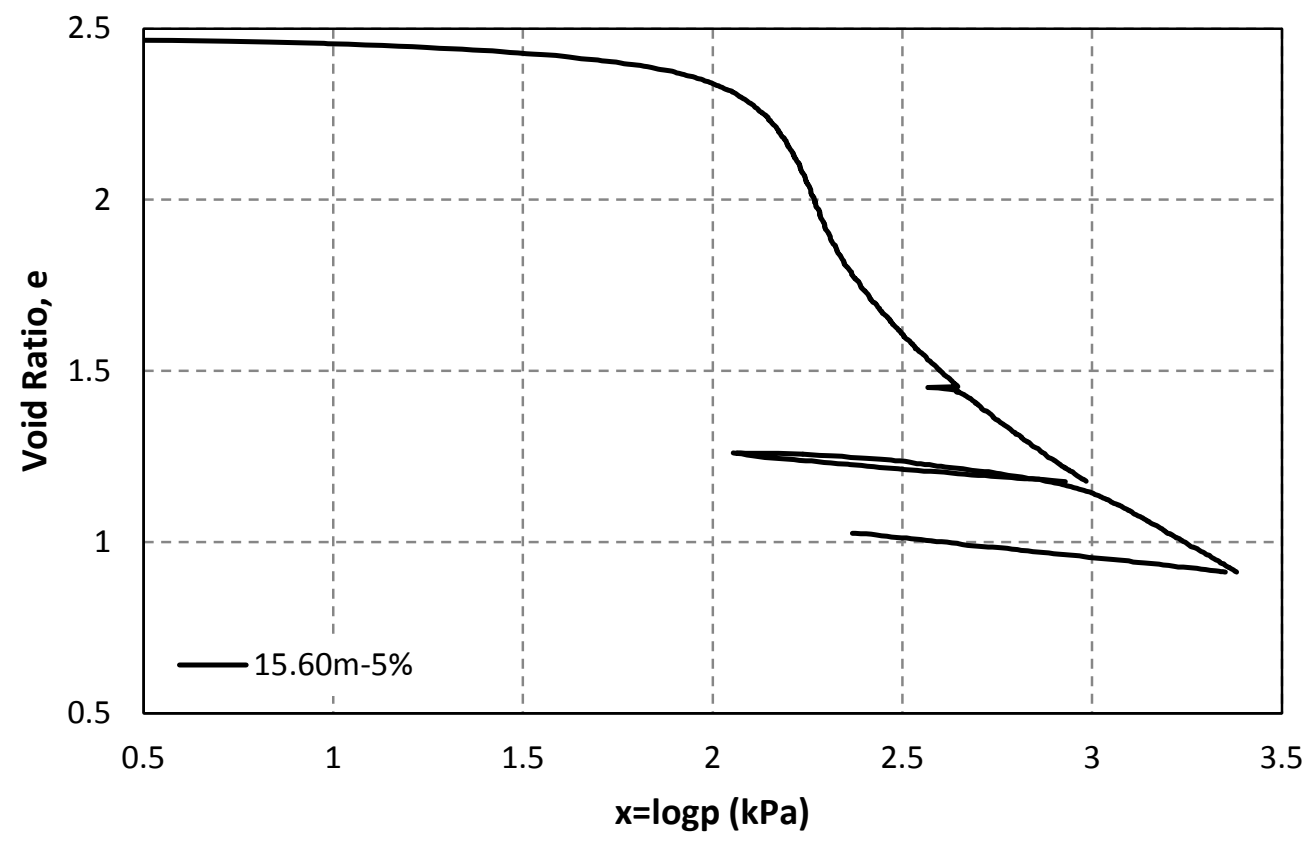

Figure 3-13: e-logp plot from 15.60m-5\% test

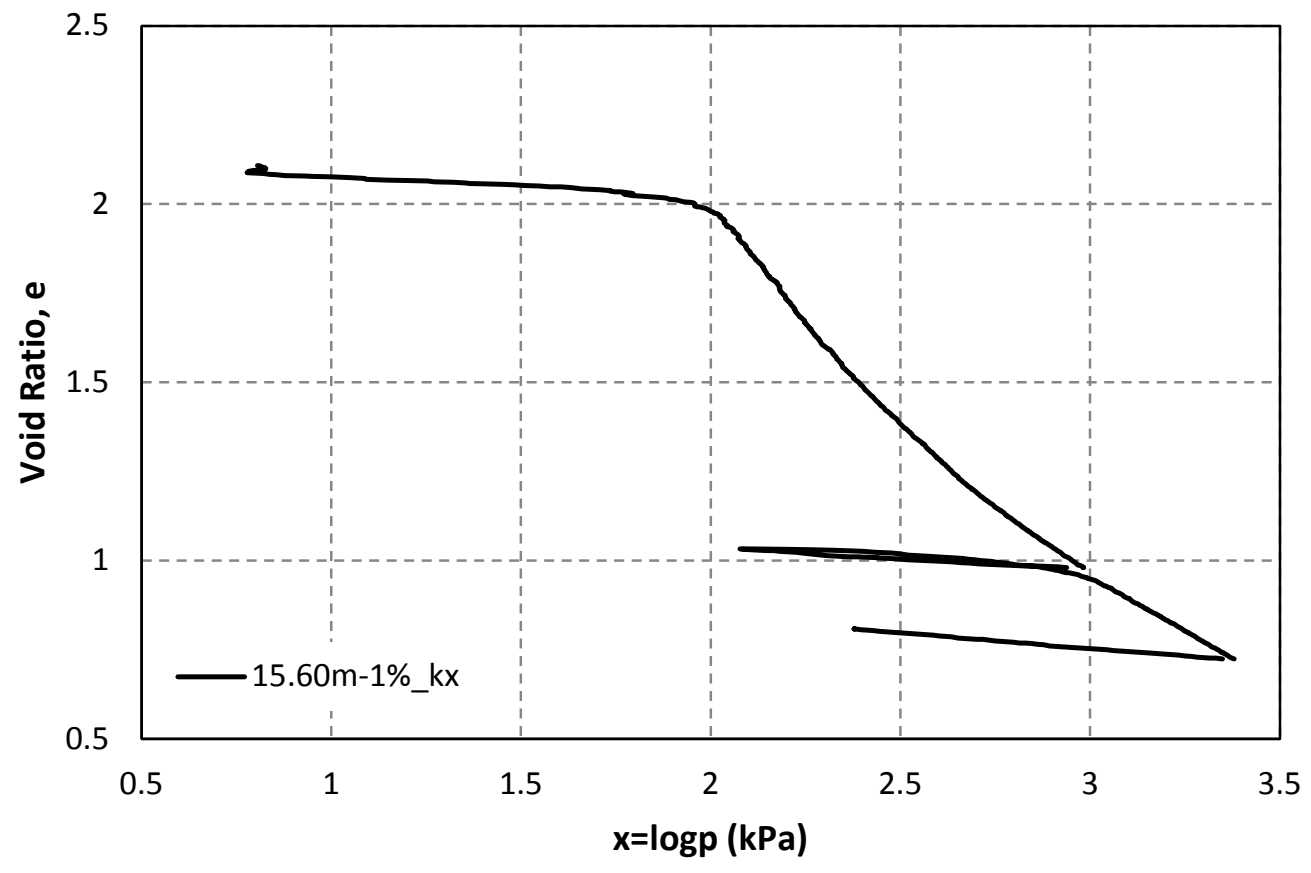

Figure 3-14: e-logp plot from 15.60m-1\%_kx test 


\subsubsection{Samples from $30.48 \mathrm{~m}$}

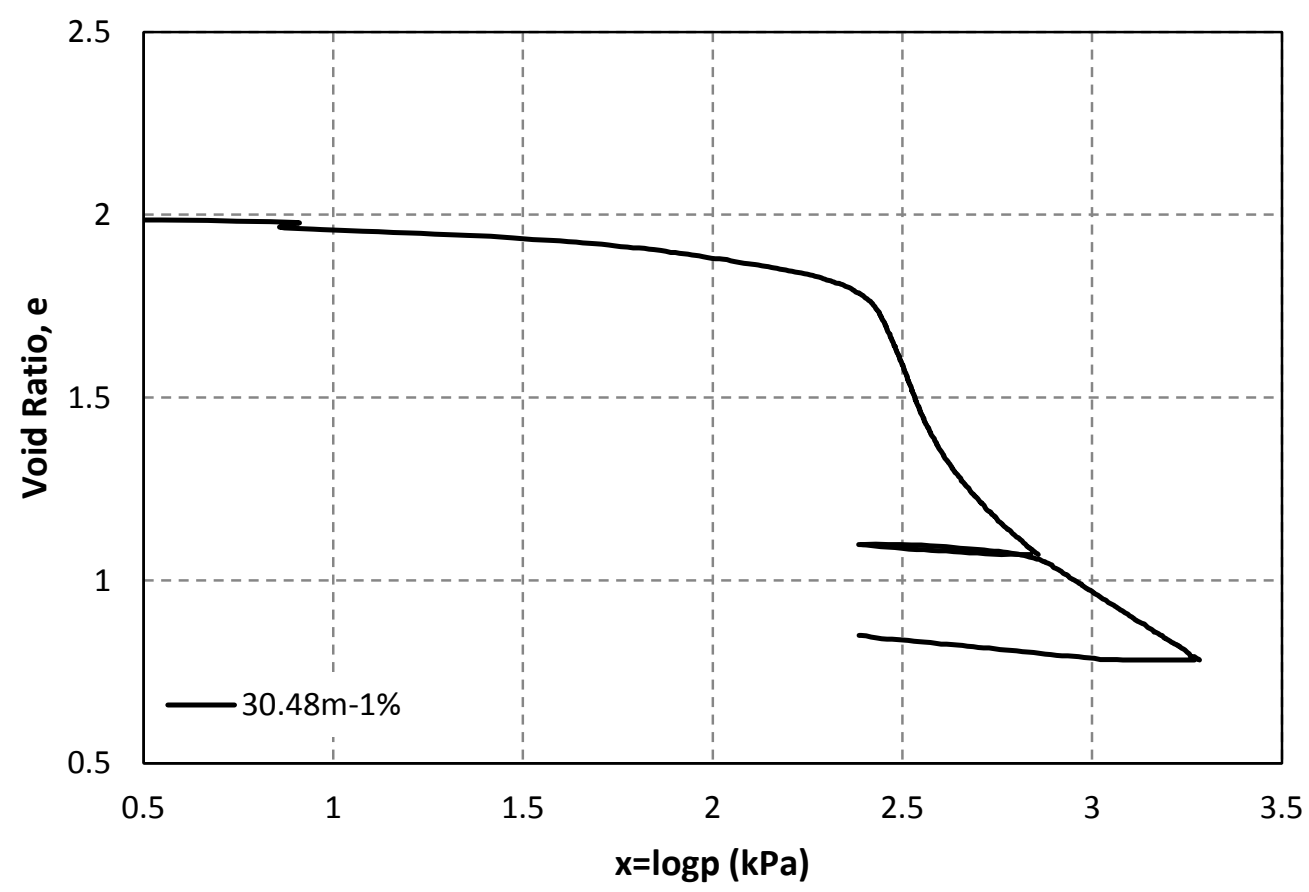

Figure 3-15: e-logp plot for 30.48m-1\% test

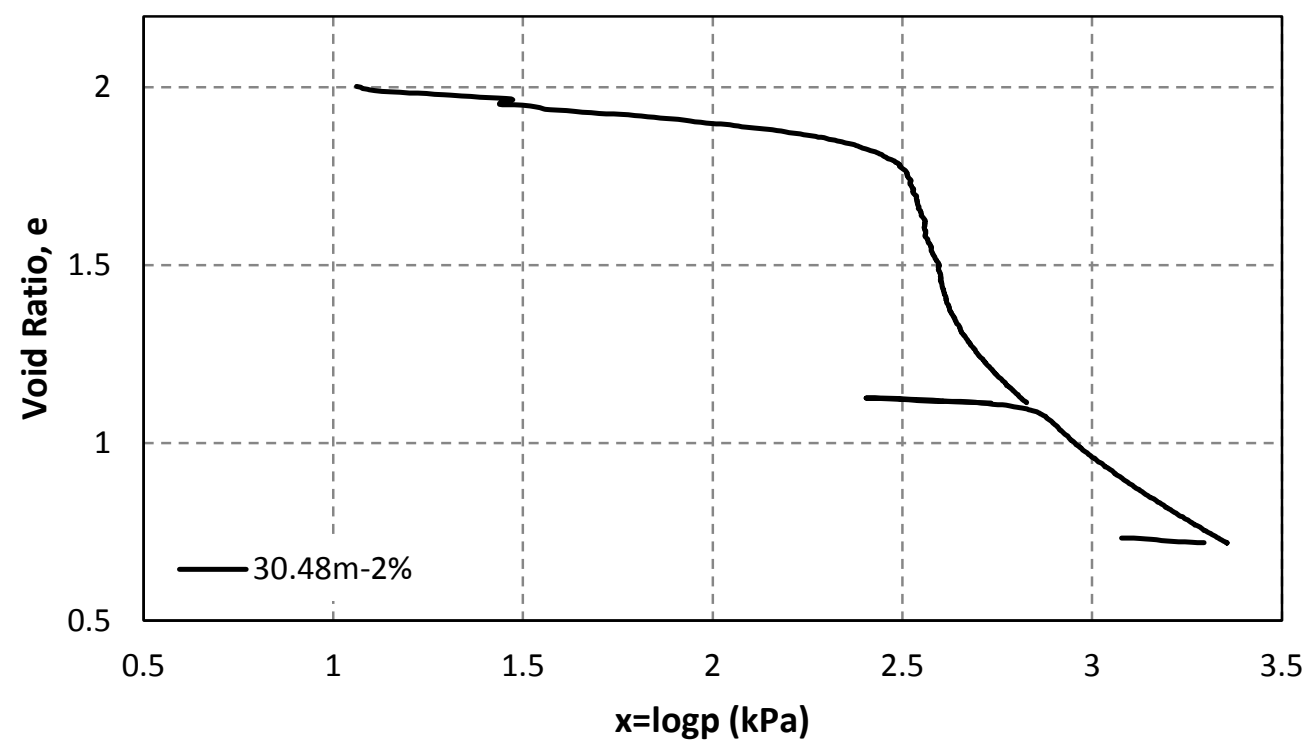

Figure 3-16: e-logp plot for 30.48m-2\% test 


\subsubsection{Sample from $34.90 \mathrm{~m}$}

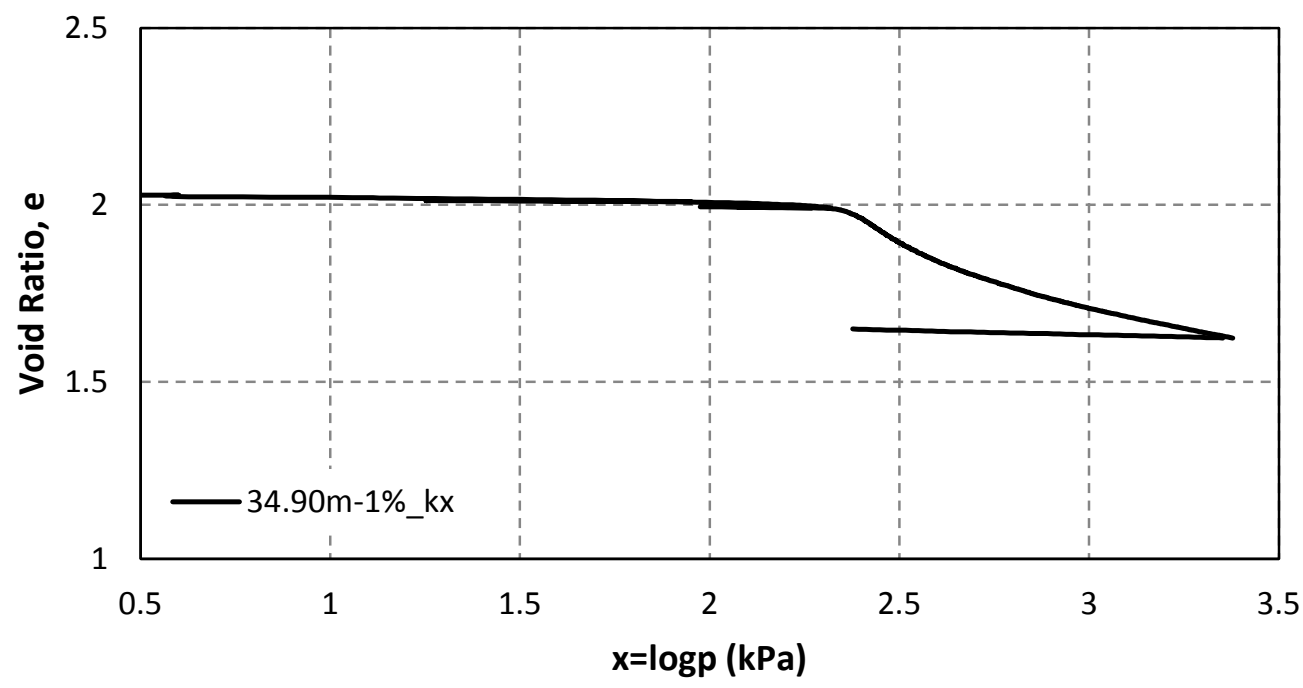

Figure 3-17: e-logp plot for 34.90m-1\%_kx test

\subsubsection{Samples from $39.48 \mathrm{~m}$}

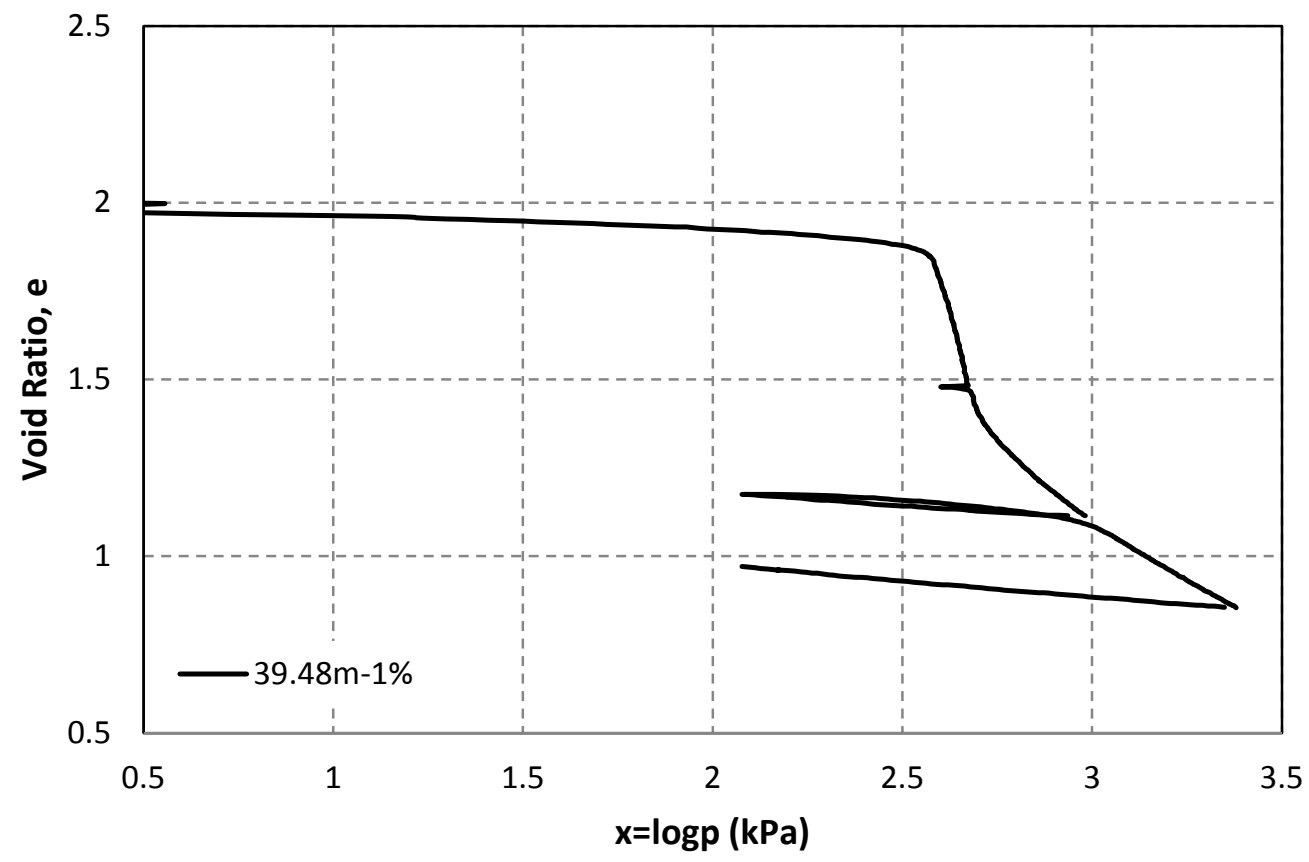

Figure 3-18: e-logp plot for 39.48m-1\% test 


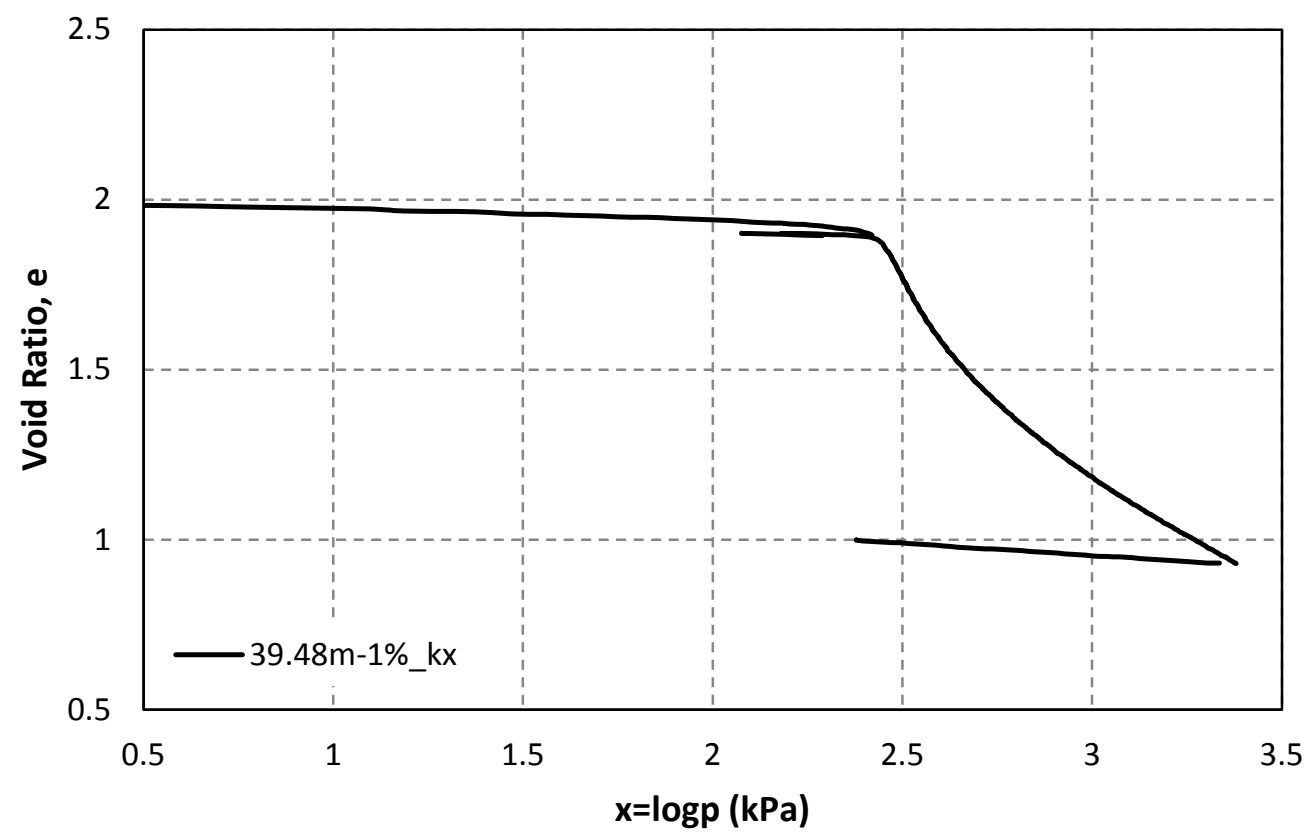

Figure 3-19: e-logp plot from 39.48m-1\%_kx test

\subsection{SUMMARY OF CRS TESTS}

The CRS test is a rapid way to perform consolidation tests, and is commonly used for sensitive soils such as Champlain Sea clay. 11 tests were performed on samples from the Waba Dam site for the determination of preconsolidation pressure by use of the different graphical methods. Nine of the tests included reloading stages at sufficient unloading stresses such that the graphical methods could be used. In all tests, the expected "S-shape" of the $e$-logp curve was obtained. Due to other project needs, three of the tests were performed on samples that were prepared so that their orientation during testing was transverse to the orientation underground. 


\section{NUMERICAL TECHNIQUES TO AID IN GRAPHICAL CONSTRUCTION METHODS}

One of the major criticisms of most existing methods to determine preconsolidation pressure is that they are graphical in nature. This reduces the objectivity of the methods, and there is no guarantee that the same result will be obtained when a procedure is repeated on the same data.

A characteristic of the CRS test is the collection of a very large number of data points with which to construct the consolidation curve. This facilitates the application of simple numerical techniques to obtain the point of maximum curvature and the inflection point of the e-logp curve with more precision. Furthermore, values such as the slope of the curve at the point of maximum curvature or at the inflection point can be obtained. Since these values are now based solely on the data without relying on user interpretation, this reduces the subjectivity of the graphical methods.

The potential for user error can be further reduced by using straight line equations to plot the lines required for the graphical constructions rather than drawing them by hand. This allows a great deal of control for the user in how to place the lines. The intersection of these straight line equations can then be calculated to determine the preconsolidation pressure. This has the added benefit of eliminating the need to interpret effective stress on a logarithmic scale (Boone, 2010). It should be mentioned that drawing the lines in this way does not change the graphical nature of the methods to determine preconsolidation pressure.

The numerical procedures to determine the points of maximum curvature and inflection point and the slope of the curve at those points will be outlined in the following sections. Following this, the methods to determine preconsolidation pressure as the intersection of straight line equations will be presented.

\subsection{Locating Key Points On The E-LogP CURVE}

\subsubsection{Locating Point of Maximum Curvature}

The curvature of a function $f(x)$ is defined by the following formula:

$$
\kappa(x)=\frac{\left|f^{\prime \prime}(x)\right|}{\left[1+f^{\prime}(x)^{2}\right]^{3 / 2}}
$$

In order to apply this formula to e-logp data, the following substitution must be made: 


$$
x=\log p
$$

A numerical approximation of the curvature formula is made by substituting the first derivative for the slope between two points as e' $\left(\mathrm{x}_{\mathrm{i}}\right)$ and the second derivative for the second slope e' $\left(\mathrm{x}_{\mathrm{i}}\right)$ :

$$
\begin{aligned}
e^{\prime}\left(x_{i}\right) & =\frac{e_{i+1}-e_{i-1}}{x_{i+1}-e_{i-1}} \\
e^{\prime \prime}\left(x_{i}\right) & =\frac{e^{\prime}{ }_{i+1}-e^{\prime}{ }_{i-1}}{x_{i+1}-e_{i-1}}
\end{aligned}
$$

Due to scatter in the data, the slope between two adjacent points can potentially be much larger or smaller than the average slope of the curve at a given point. Thus, the average slope must be estimated over a certain range of points on either side of the point of interest. This can be expressed as:

$$
e^{\prime}\left(x_{i}\right)=\frac{e_{i+\Delta i}-e_{i-\Delta i}}{x_{i+\Delta i}-x_{i-\Delta i}}
$$

where $2 * \Delta i+1$ is the range of points over which the slope is calculated.

If the range is chosen that is too large, the slope that is calculated will also not be representative of the average slope at any given point, as the line will behave like a secant and deviate from the data. Thus, an optimized range must be selected that sufficiently accounts for the scatter in the $e$ logp data but still represents the average slope of the curve.

In the figure on the left below, the effect of choosing a range that is too large is shown. The slope at any given data point is shown as the secant connecting the "i" points away on either side. The range in the left figure was 61 data points. For the data sets experienced in the current study, a range of 21 data points was found to be appropriate to both smooth the data and avoid deviating from the $e$-logp curve. This is shown in the figure on the right below. 

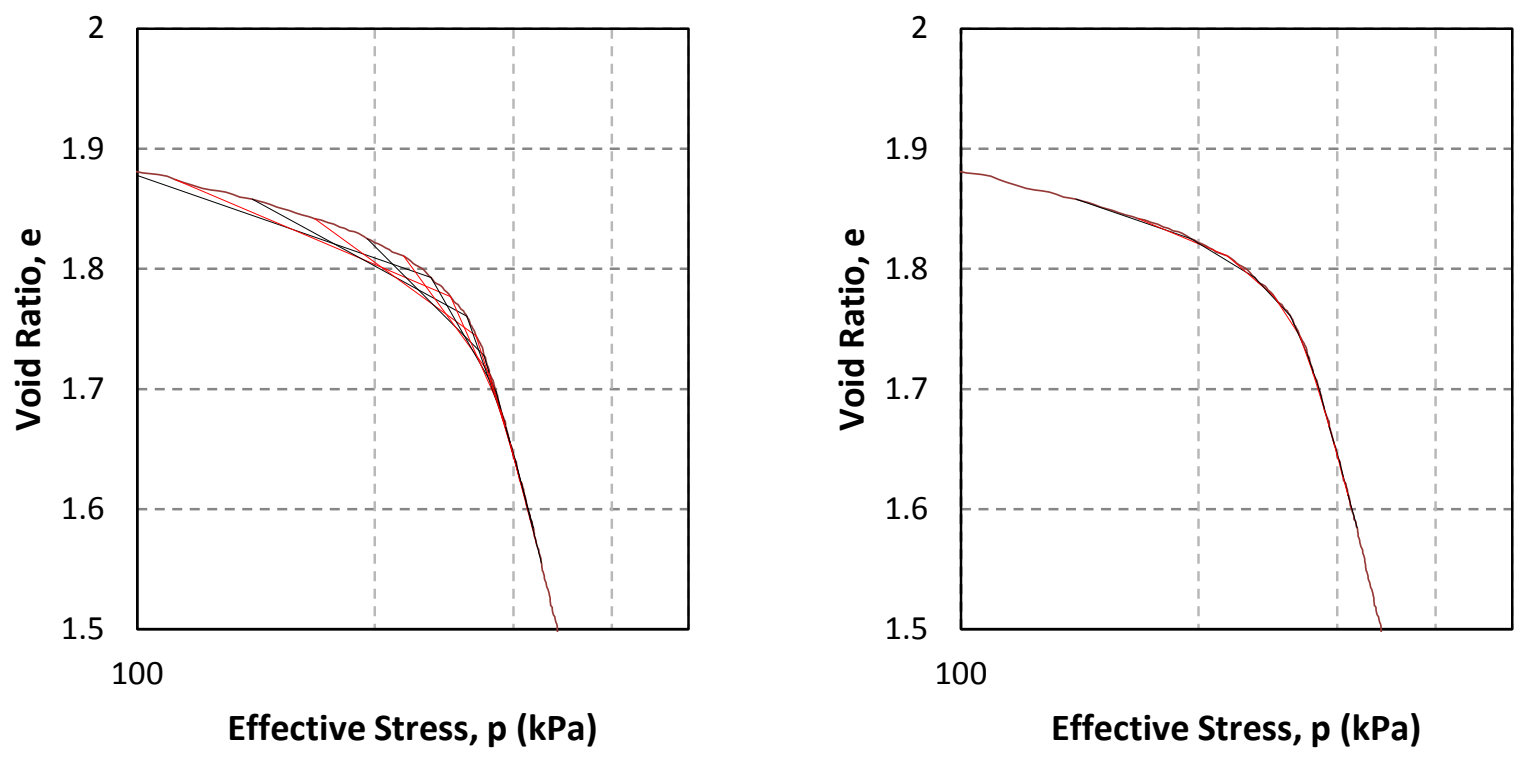

Figure 4-1: Selection of an appropriate range when calculating average slope of an $e$-logp curve Once the average slope has been calculated for all data points, it is possible to plot the development of the slope for every value of effective stress during the consolidation test. An example is shown in Figure 4-2.

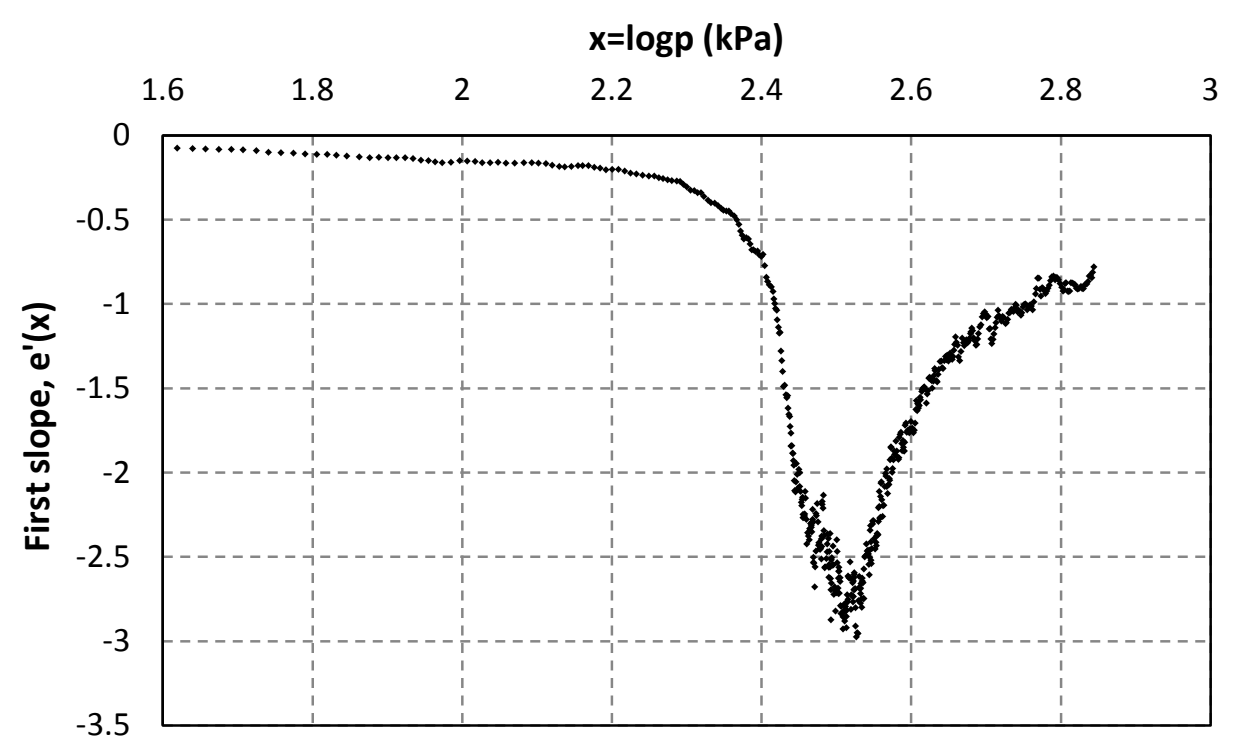

Figure 4-2: Development of first slope of $e$-logp curve throughout a typical CRS test

From here, the second slope of the $e$-logp curve can be determined by calculating the slope between two points on the slope curve. This can be expressed using the formula: 


$$
e^{\prime \prime}\left(x_{i}\right)=\frac{e^{\prime}{ }_{i+\Delta i}-e^{\prime}{ }_{i-\Delta i}}{x_{i+\Delta i}-x_{i-\Delta i}}
$$

It is necessary, once again, to determine the above as the average slope over a range of data points. Once again, a range of 21 data points was found to be appropriate. Because of the large number of data points obtained in a CRS test, the relatively small number of points lost at the extreme ends of the data set is not significant. The resulting curve for the same data set is shown in Figure 4-3.

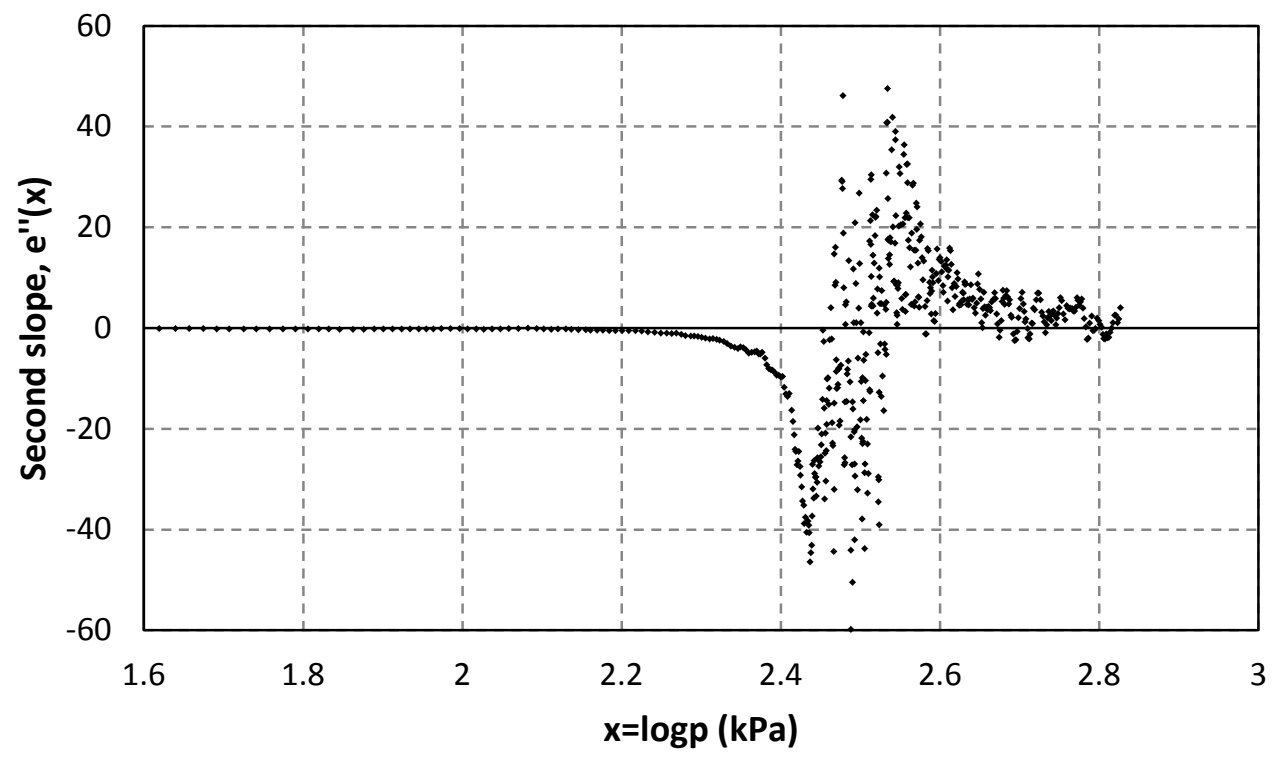

Figure 4-3: Development of second slope of e-logp curve throughout a CRS test

Now that $\mathrm{e}^{\prime}(\mathrm{x})$ and $\mathrm{e}^{\prime \prime}(\mathrm{x})$ have been found for each data point, they can be substituted into the curvature formula:

$$
\kappa\left(x_{i}\right)=\frac{\left|e^{\prime \prime}\left(x_{i}\right)\right|}{\left[1+e^{\prime}\left(x_{i}\right)^{2}\right]^{3 / 2}}
$$

The resulting plot of curvature is: 


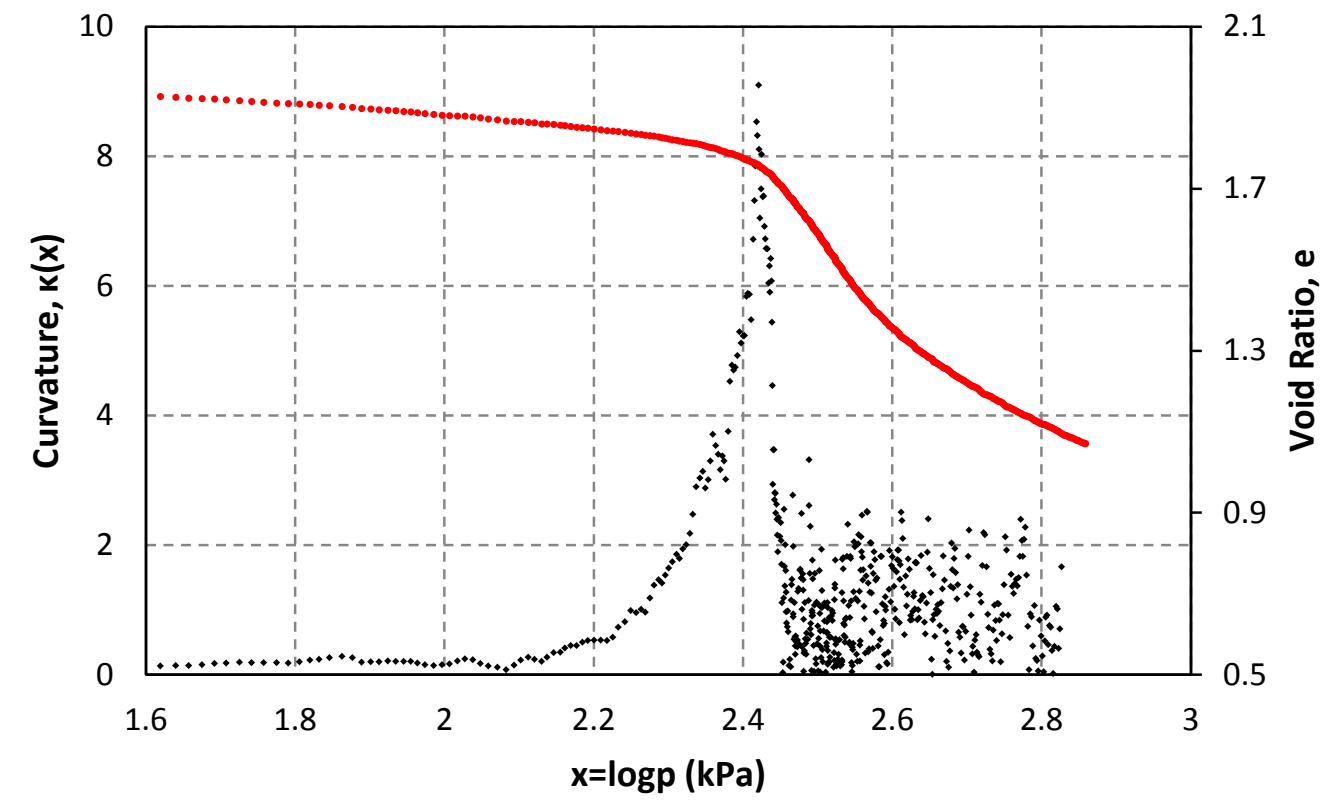

Figure 4-4: Use of Curvature Formula to Determine Point of Maximum Curvature

From the above plot, it is easy to obtain the point of maximum curvature of the $e$-logp curve, as the curvature is seen to have a clear peak.

It has been mentioned by numerous authors that the selection of vertical scale with respect to the horizontal scale has an influence on the value of the point of maximum curvature ( $\mathrm{Li}, 1989)$ (Clementino, 2005) (Boone, 2010). The use of this formula does not solve the problem of scale effects on the selection of this point. However, it does provide a means to quantify the influence. This can be seen in Figure 4-5, where curvature was calculated and plotted using the same set of data, except that in one of the cases, the void ratio column was multiplied by a constant 4 . 


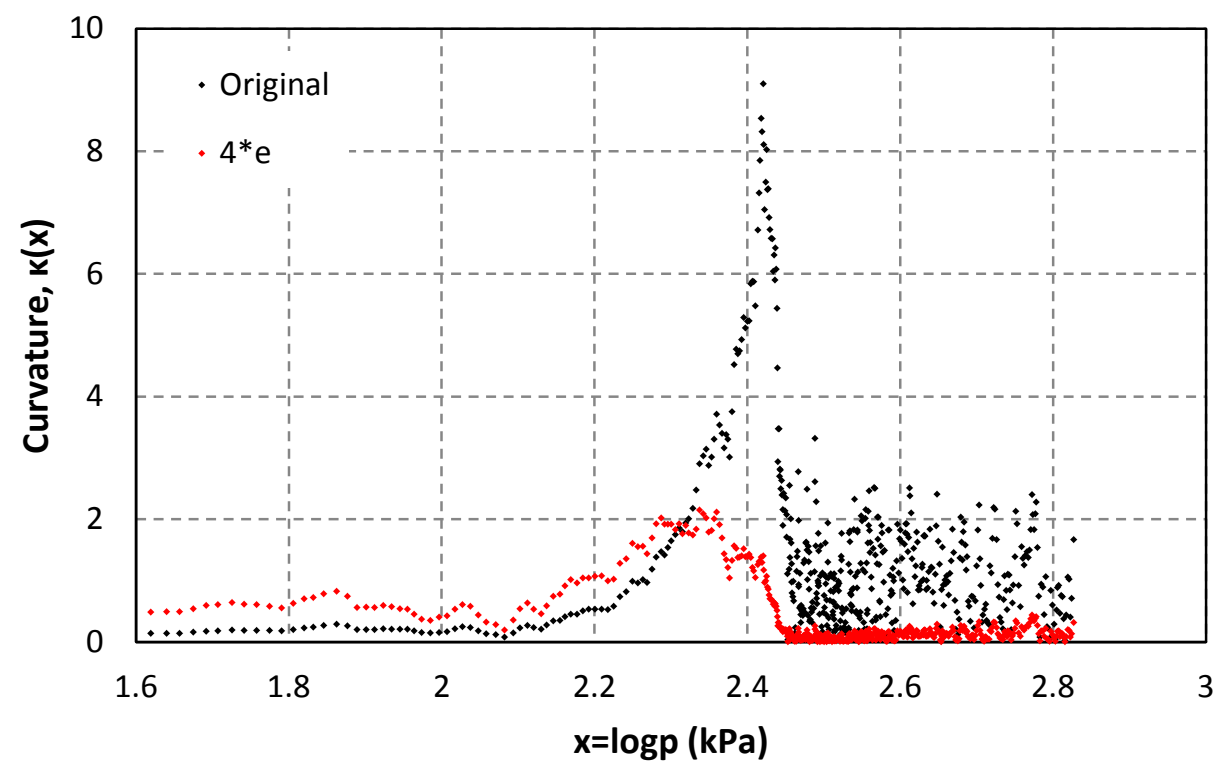

Figure 4-5: Comparison of curvature plots for different vertical scale factors

As can be seen from the above figure, exaggerating the vertical scale reduces the absolute value of curvature at the point of maximum curvature. More significantly, the stress at which maximum curvature occurs is lower.

Although the use of the curvature formula does not solve the problem of scale effects, it can still be considered to provide an objective evaluation of the point of maximum curvature for a selected scale ratio. Unless a scale factor is applied, a ratio of e:logp $=1: 1$ is assumed.

\subsubsection{Locating Inflection Point}

The inflection point of a function is defined as a point where the function changes from concave down to concave up, or vice versa. Because of the characteristic "S-shape" of the e-logp curve of sensitive clay, these soils are known to exhibit such a point. In calculus, the inflection point of a function can be found when the second derivative is equal to zero, or when the first derivative reaches a local maximum or minimum. This concept can be applied to consolidation data by calculating the slope of the consolidation curve at every point, then locating the maximum or minimum value.

In Figure 4-6, the slope of the curve is plotted against logarithm of effective stress. The slope is denoted as e'(x). It can be noted that the slope of the $e$-logp curve has a negative value due to the tendency of void ratio to decrease with the application of stress. Furthermore, the inflection point is a minimum on this plot because the $e$-logp curve changes from concave down to concave up rather than vice versa. 


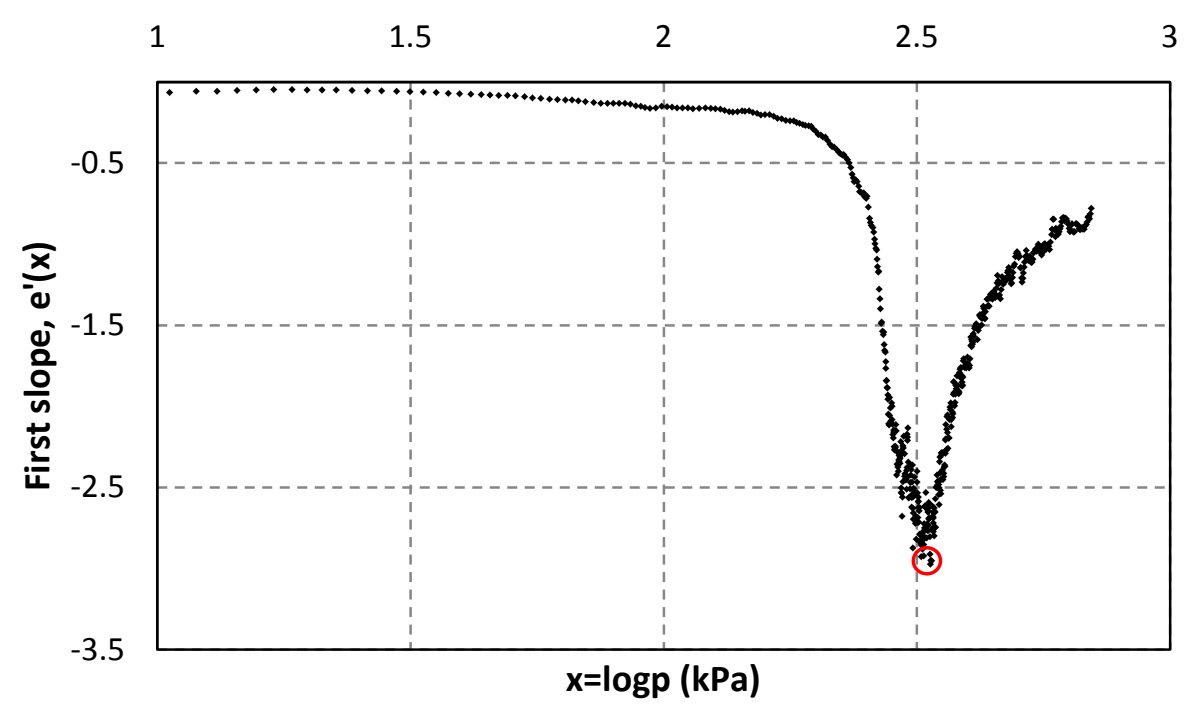

Figure 4-6: Determination of inflection point as point of maximum slope

The locations of maximum curvature and inflection point as obtained by these numerical methods can now be used to perform the traditional graphical construction procedures to determine preconsolidation pressure with less subjectivity.

\subsection{Numerical Approaches to Graphical Methods}

\subsubsection{Numerical-Aided Casagrande Method}

In order to perform the Casagrande method without graphical interpretation, the following steps are taken:

1) Locate the point of maximum curvature using the formula:

$$
\kappa(x)=\frac{\left|e^{\prime \prime}(x)\right|}{\left[1+e^{\prime}(x)^{2}\right]^{3 / 2}}
$$

2) Determine the slope of the curve at the point of maximum curvature.

3) Plot a line with the slope of the consolidation curve at maximum curvature, using the coordinates of the point of maximum curvature as boundary conditions:

$$
e=e^{\prime}\left(x_{M C}\right) * x+\left[e_{M C}-e^{\prime}\left(x_{M C}\right) * x_{M C}\right]
$$

4) Plot a straight horizontal line at the point of maximum curvature 


$$
e=e_{M C}
$$

5) Plot the line that bisects the angle between the two lines plotted previously. The slope of this line is:

$$
\tan \left(\frac{\alpha}{2}\right)=\frac{-1}{\tan \alpha}+\left(\frac{1}{\tan \alpha}\right) * \sqrt{(}\left(1+(\tan \alpha)^{2}\right)
$$

6) Draw a line using the slope of the consolidation curve at the inflection point and the coordinates of the curve at the point as the boundary conditions:

$$
e=e^{\prime}\left(x_{i n f}\right) x+\left[e_{i n f}-e^{\prime}\left(x_{i n f}\right) x_{i n f}\right]
$$

7) Calculate the intersection of the bisecting line and the inflection point line to obtain the logarithm of effective stress at the Casagrande p'c:

$$
x_{p \prime c}=\frac{\left[e_{i n f}-e_{M C}\right]-\left[e^{\prime}\left(x_{i n f}\right) * x_{i n f}-e^{\prime}\left(x_{M C}\right) * x_{M C}\right]}{e^{\prime}\left(x_{i n f}\right)-e^{\prime}\left(x_{M C}\right)}
$$

8) Calculate the effective stress at the intersection:

$$
p_{c}^{\prime}=10^{x_{p \prime c}}
$$

In the above formulas, the subscript "MC" denotes maximum curvature and the subscript "inf" denotes inflection point.

An example of this procedure is shown in Figure 4-7. The scales are intentionally drawn such as to reflect the assumed e:logp = 1:1 ratio. 


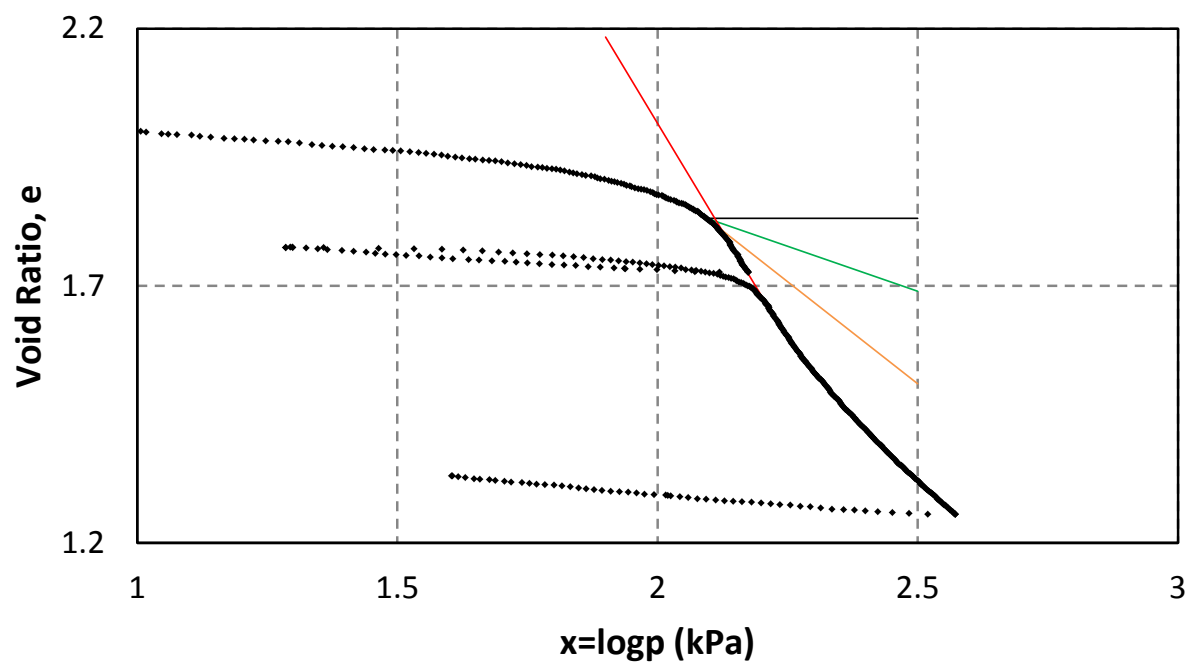

Figure 4-7: Example of the use of formulas to perform Casagrande construction

It can be observed from the above figure that the preconsolidation pressure as obtained by Casagrande construction is very close to the point of maximum curvature. By adjusting the scales of the axes, one can see more clearly the location of p'c relative to the consolidation curve.

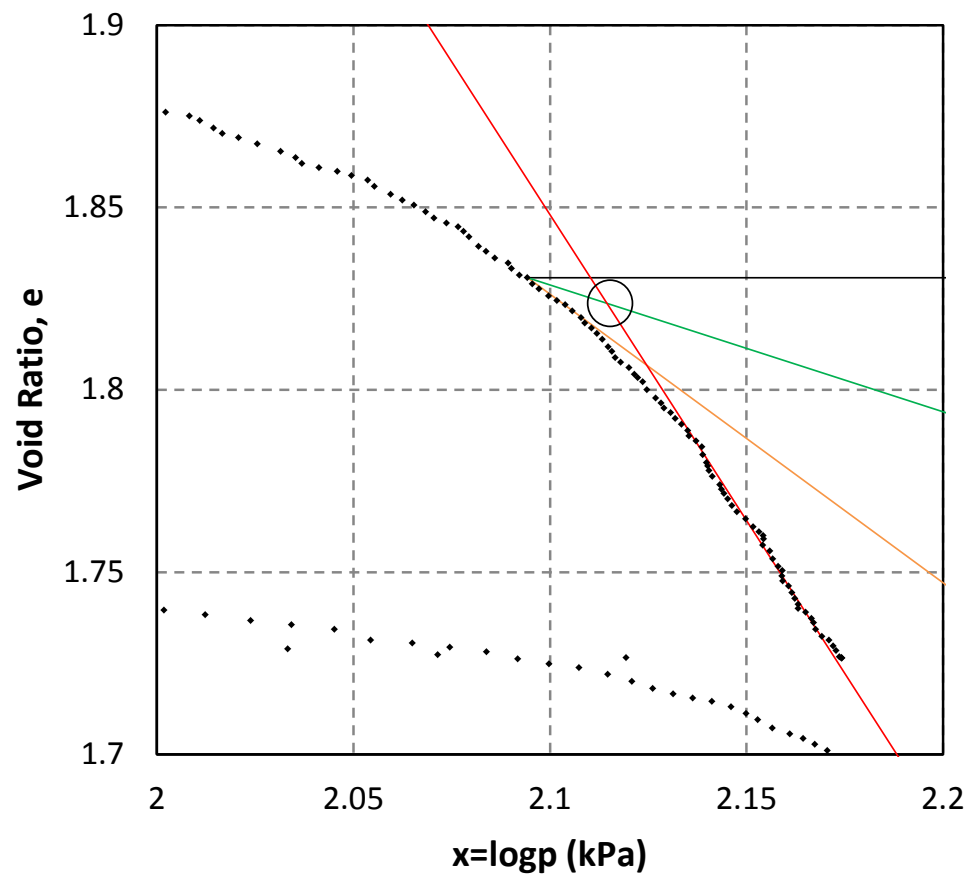

Figure 4-8: Adjusted scales to show location of p'c from Casagrande construction 


\subsubsection{Numerical-Aided Nagaraj et al. Method}

The location and value of the slope of the consolidation curve at the point of maximum curvature can also be used to perform the method of Nagaraj et al. (1990). The steps for this procedure are as follows:

1) Determine the location and slope of the point of maximum curvature as described previously

2) For illustration purposes, draw the tangent line to the consolidation curve at the point of maximum curvature as previously determined.

3) Draw a line with the inverse slope of the consolidation curve at maximum curvature, using the coordinates of the point of maximum curvature as boundary conditions:

$$
e=\frac{-1}{e^{\prime}\left(x_{M C}\right)} * x+\left[e_{M C}+\frac{1}{e^{\prime}\left(x_{M C}\right)} * x_{M C}\right]
$$

4) Draw a straight horizontal line with the value of the initial void ratio:

$$
e=e_{0}
$$

5) Calculate the intersection of these lines:

$$
x_{p_{c}^{\prime}}=x_{M C}+e^{\prime}\left(x_{M C}\right) *\left[e_{M C}-e_{0}\right]
$$

6) Calculate the corresponding effective stress:

$$
p_{c}^{\prime}=10^{x} p_{c}^{\prime}
$$

An example of this procedure is shown in Figure 4-9. 


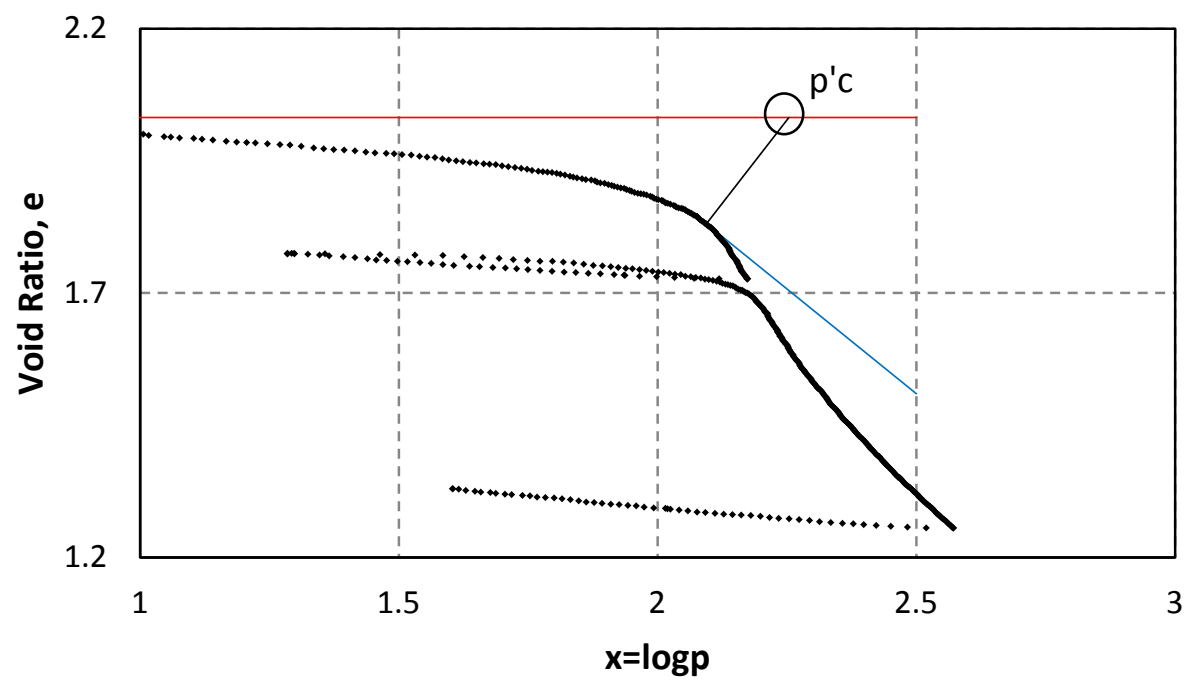

Figure 4-9: Example of the use of formulas to perform Nagaraj et al. (1990) method

It should be noted that the angle between the tangent line and the inverse tangent line will only appear as perpendicular if a 1 to 1 ratio of horizontal to vertical scale is used.

\subsubsection{Numerical-Aided Work Method}

The Work method (i.e. Total Strain Energy method or Becker et al. (1987) method) can also be implemented using the same concepts that have been described, . This can be done according to the following steps. Due to the similarity with the previous methods, the formulas will not be repeated.

1) Calculate the slope $W$ - $p$ curve both before and after yield

2) Plot straight lines using these slopes and points on the $W-p$ curve as boundary conditions

3) Calculate the intersection of the two straight lines to obtain the preconsolidation pressure An example of this procedure is shown in Figure 4-10. 


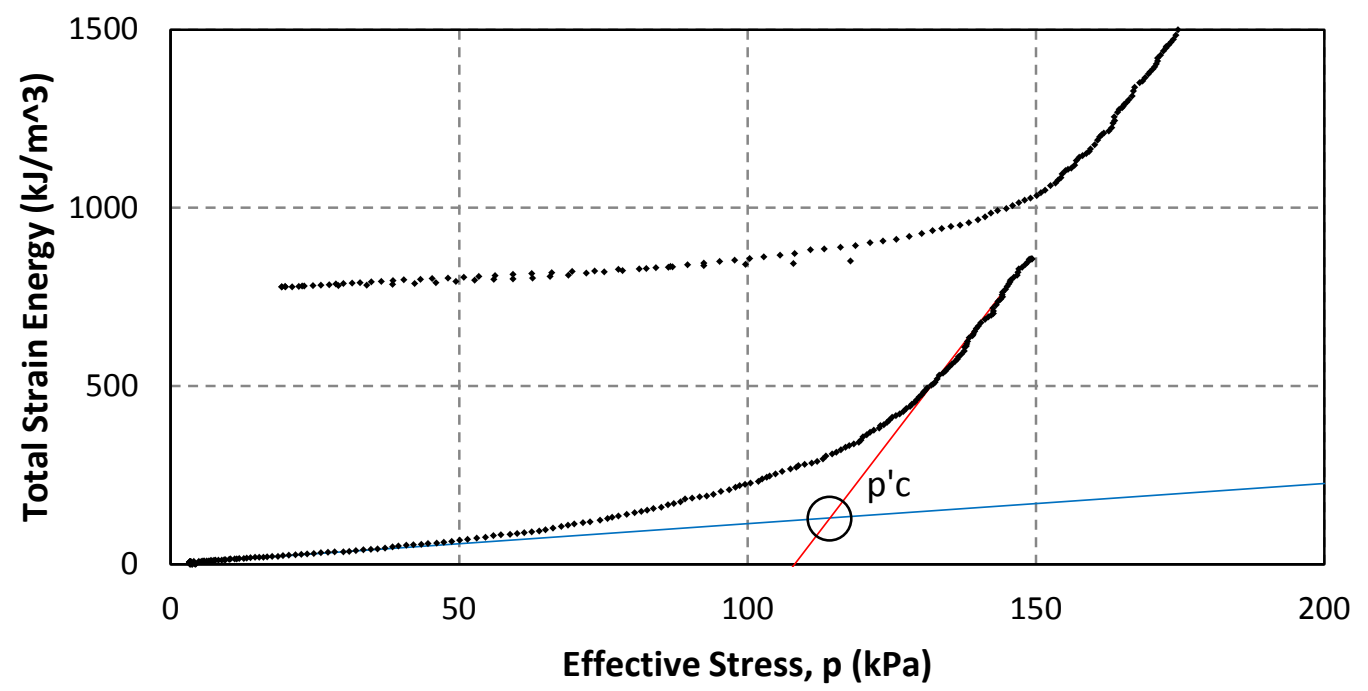

Figure 4-10: Example of the use of formulas to perform work method

\subsubsection{Numerical-Aided Wang and Frost Method}

The plot that was created to perform the Work method can be updated to perform the Dissipated Strain Energy method (i.e. Wang and Frost method). This is done as follows:

1) Extend the post-yield line to intersect with the vertical axis at $x=0$ and draw a horizontal line having the value of total strain energy at the intersection.

2) Draw a line having the slope of the recompression part of the $W$-p plot, using the coordinates of the point where the post-yield line intersects the vertical axis as a boundary condition.

3) Choosing an arbitrary location on the horizontal x-axis, calculate the difference between the horizontal line

In order to see the preconsolidation pressure more clearly, the scales are adjusted in Figure 4-11. Additionally, the pre-yield line is once again added to show the preconsolidation pressure as determined by the Work method for comparison. In this example, the two methods gave nearly identical values for preconsolidation pressure. 


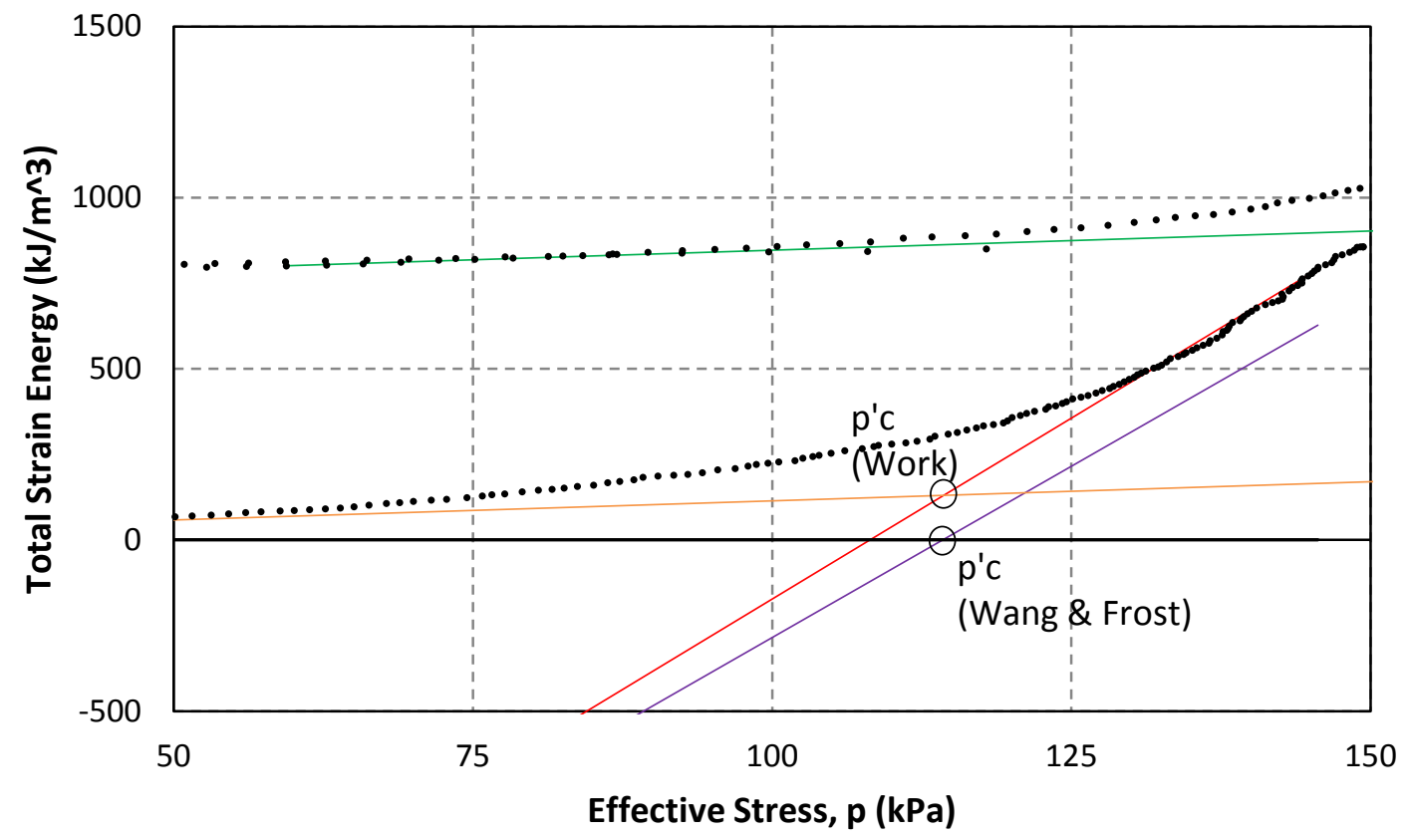

Figure 4-11: Comparison of Work and Dissipated Strain Energy (DSE) Methods using formulas

\subsubsection{Numerical-Aided Bilogarithmic and e-logp bilinear Methods}

The procedure to determine the preconsolidation pressure using the bilogarithmic methods is similar to that used for the Work method. The slopes of the pre-yield and post-yield lines in logp$\log (1+\mathrm{e})$ space are determined, then points on the consolidation curve are used as boundary conditions. The preconsolidation pressure is then found by raising 10 to the $\mathrm{x}$-value of the point of intersection. The procedures for the numerically-aided e-logp bilinear method and the $\ln (1+e)-\ln p$ method is sufficiently similar that they will not be described individually. An example of this procedure is shown in Figure 4-12. 


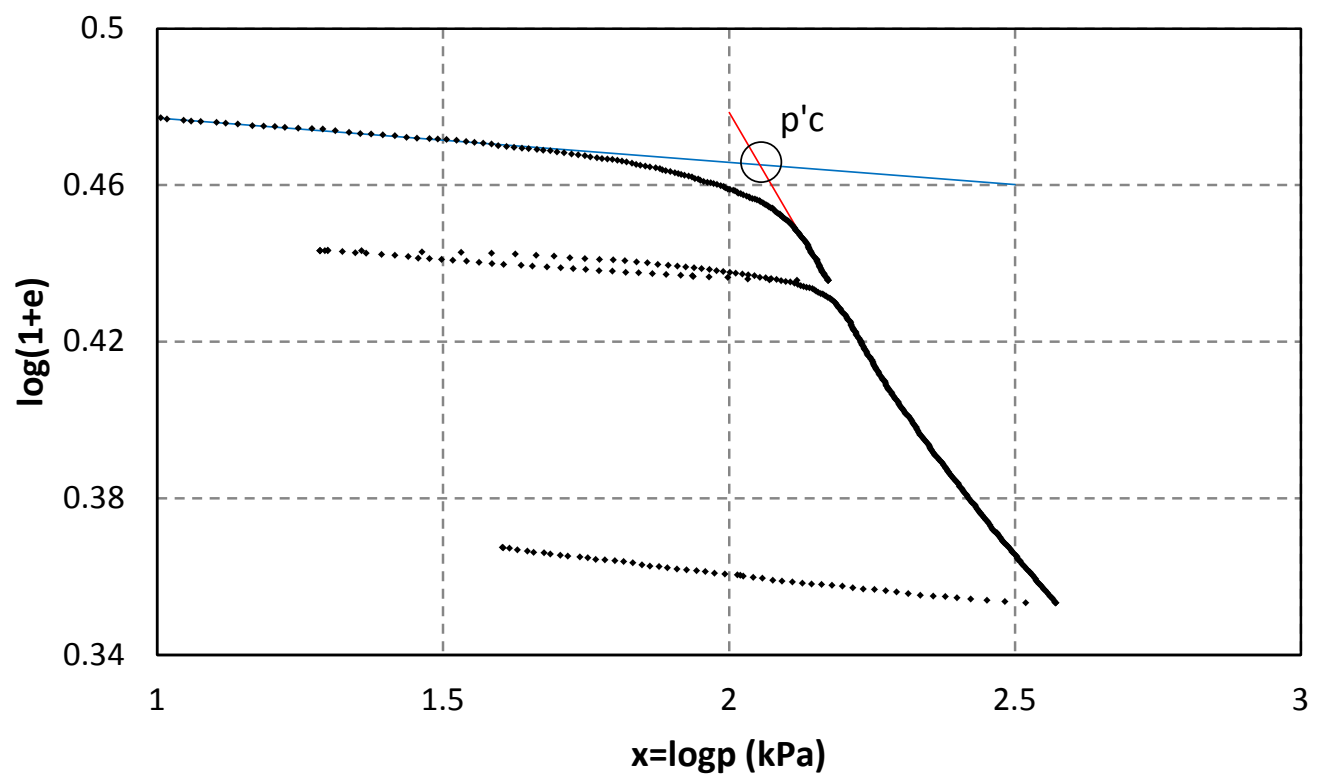

Figure 4-12: Example of the use of formulas to perform $\log (1+e)-\log p$ bilogarithmic method

\subsubsection{Numerical-Aided Pacheco Silva Method}

The method of Pacheco Silva (1970) can be performed numerically using the following procedure:

1) Determine the location and slope of the inflection point.

2) Plot a straight line using the slope and coordinates of the inflection point as boundary conditions.

3) Plot a straight horizontal line having the value $e=e_{0}$.

4) From the intersection of the two lines previously drawn, plot a straight vertical line such that it intersects the consolidation curve.

5) Select the point on the consolidation curve that is closest to the vertical line. (This is valid assuming a very large number of points constitute the consolidation curve, as is the case with a CRS test).

6) From this point, plot a straight horizontal line.

7) Calculate the intersection of the horizontal line drawn in the previous step with the line that was drawn using the slope and coordinates of the inflection point. 


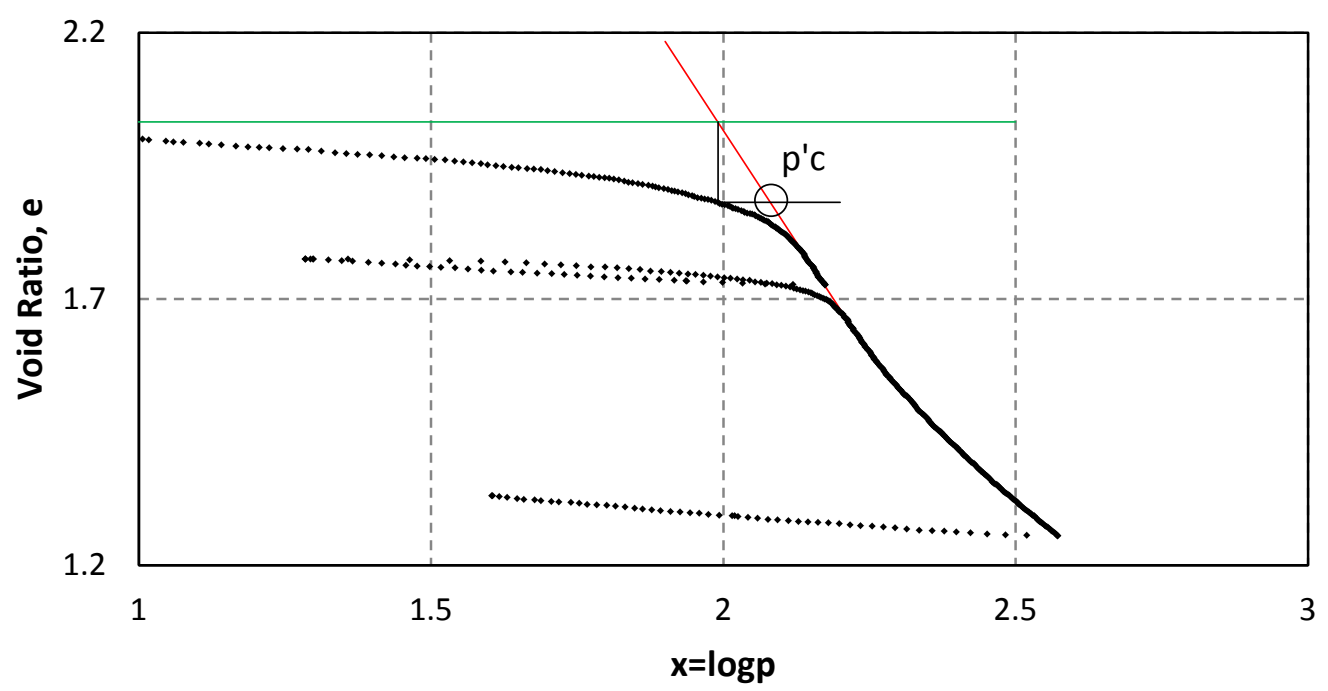

Figure 4-13: Example of the use of formulas to perform Pacheco Silva method

\subsubsection{Numerical-Aided Janbu Method}

Due to the large amount of scatter in the constrained modulus data, it is necessary to first perform some type of data smoothing before the method of Janbu (1969) can be employed. To achieve this, the median value of constrained modulus is taken over a range of 21 points. The figure below shows the effect of this data smoothing for one of the tests.

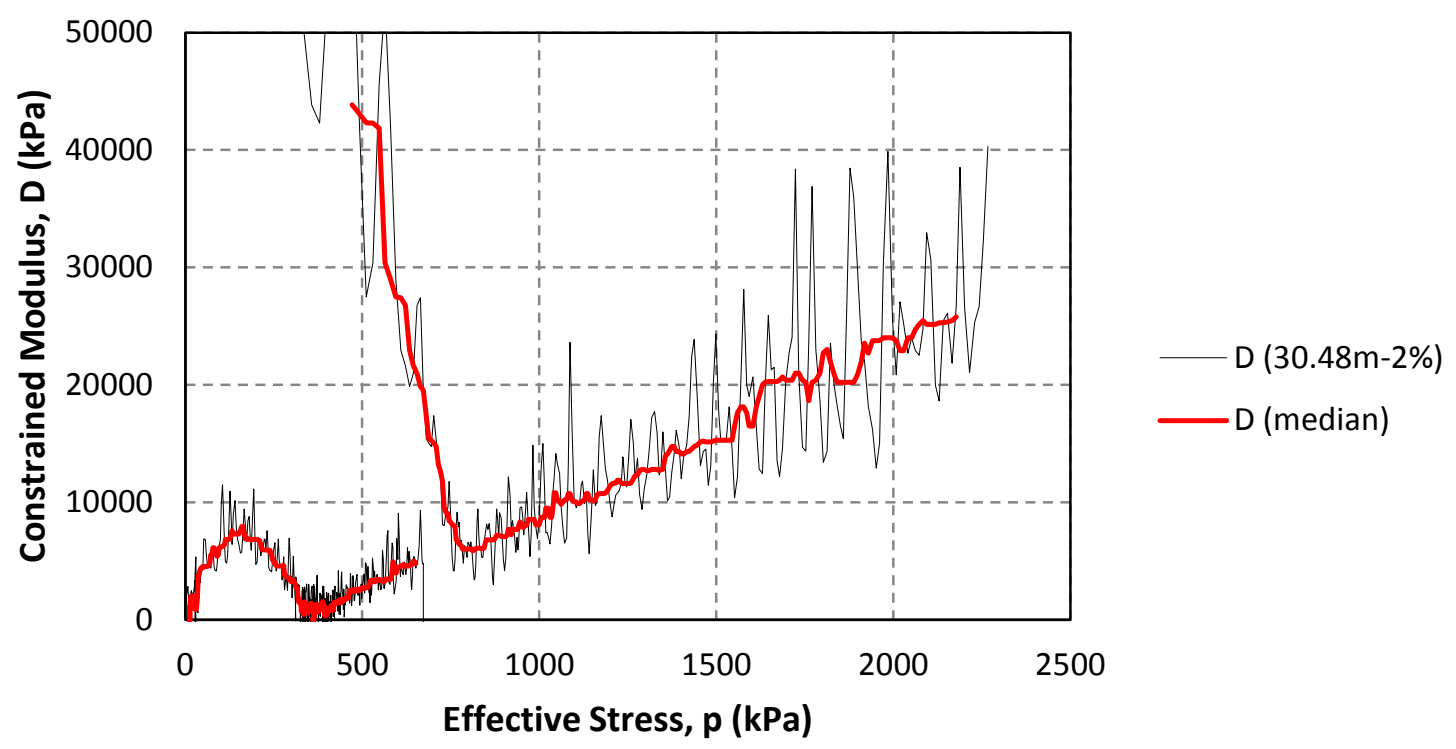

Figure 4-14: Effect of median smoothing on constrained modulus data 
Once the data has been smoothed, equations of the lines of best fit can be determined by regression. These equations are then set equal to one another to find the intersection, and the corresponding stress is the preconsolidation pressure as defined by the Janbu method. An example is shown in Figure 4-15.

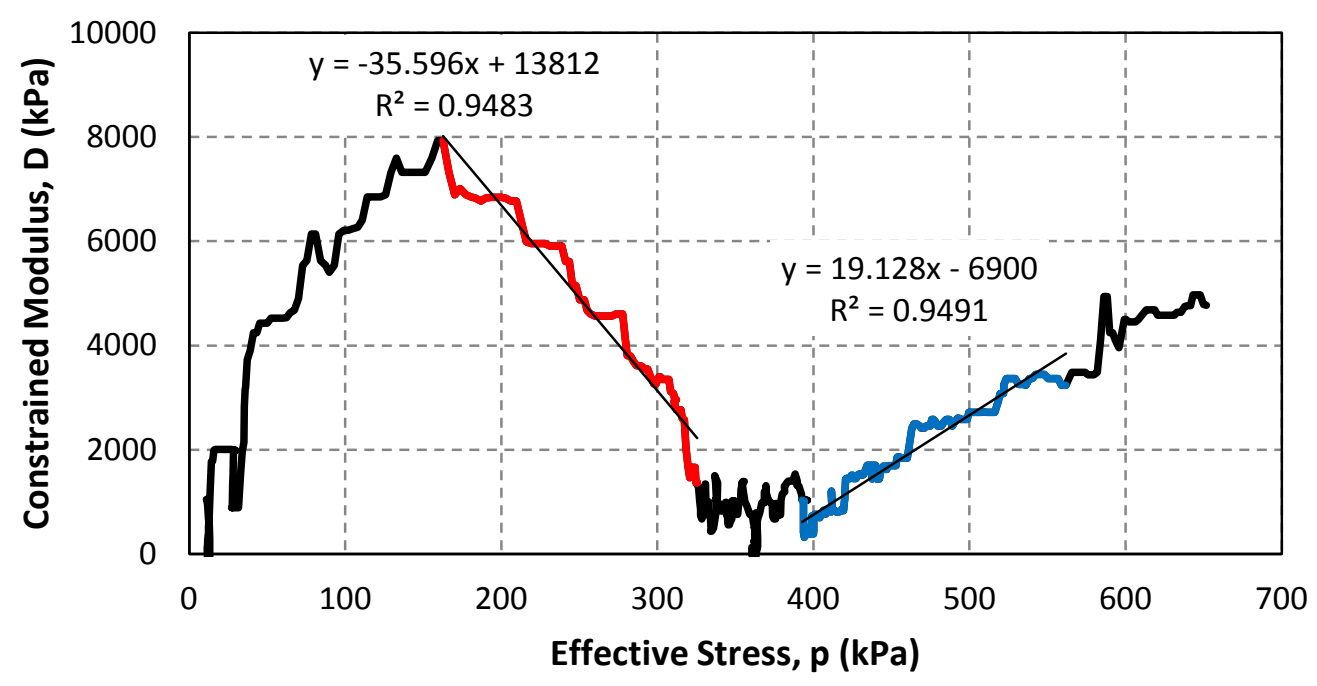

Figure 4-15: Example of obtaining lines of best fit for Janbu method

\subsubsection{Numerically-Aided Sallfors Method}

The Sallfors method is performed as follows:

1. Create a column dividing effective stress by $10\left(\mathrm{p}_{10}\right)$

2. Calculate the slope $\varepsilon-p_{10}$ curve both before and after yield

3. Plot straight lines using these slopes and points on the $\varepsilon-p_{10}$ curve as boundary conditions

4. Calculate the intersection of the two straight lines

5. Choose a tentative length for the two equal sides of the inscribed isosceles triangle

6. Based on this length, plot the two other points of the isosceles triangle and connect them to form the long edge of the triangle

7. Adjust the side length until the long edge of the triangle contacts the test curve

8. To obtain preconsolidation pressure, multiply by 10 the horizontal coordinate of the point of the triangle that falls on the pre-yield line

Some user interpretation is still required to judge when the long edge of the triangle contacts the test data. 


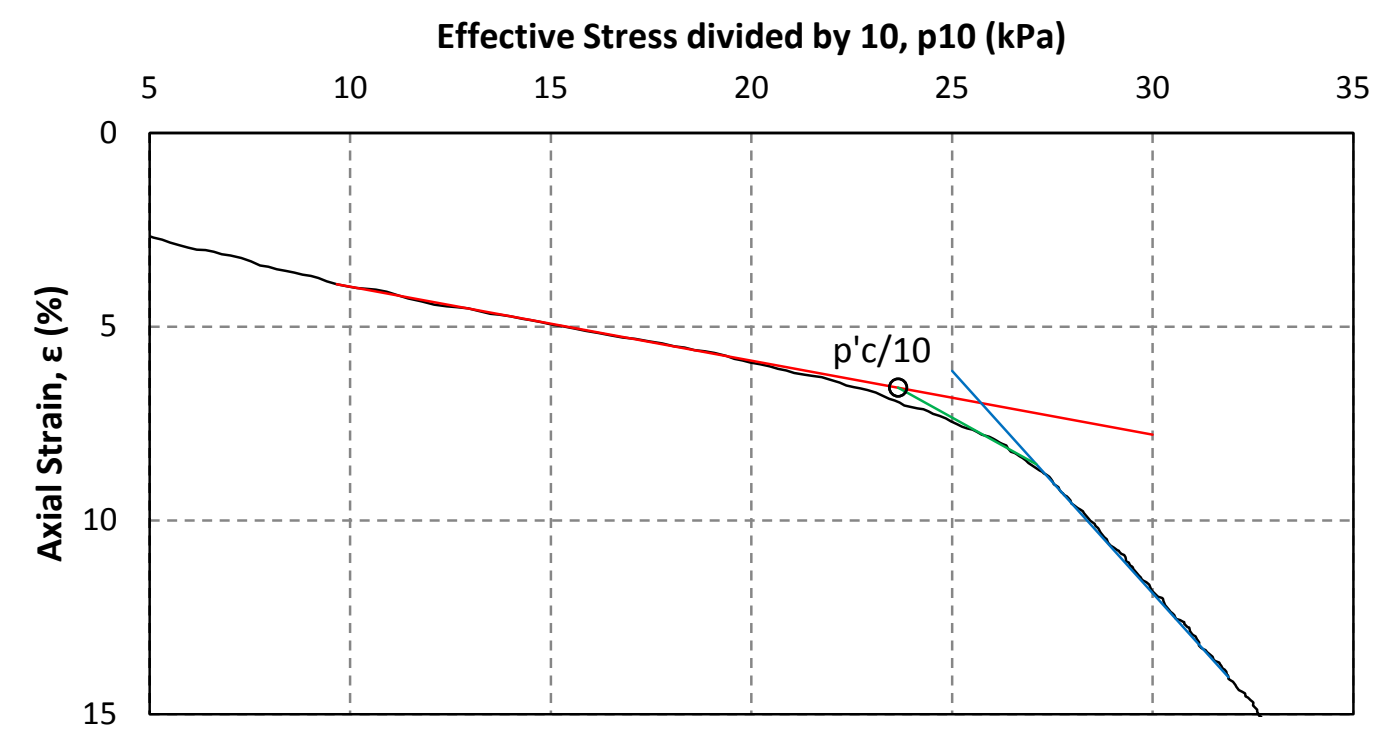

Figure 16: Example of the use of formulas to perform Sallfors method

\subsection{WORKFLOW}

Based on the above descriptions, the most convenient workflow is as follows:

1. On e-logp curve:

a. Determine the point of maximum curvature and the inflection point (maximum slope)

b. Determine the slope of the $e$-logp curve at these points

c. Perform Casagrande method using location and slope of point of maximum curvature and location and location and slope of inflection point

d. Perform Nagaraj et al. method using location and slope of point of maximum curvature

e. Perform Peck method, using location and slope of inflection point

f. Perform Pacheco Silva method using p'c found in previous step and point on consolidation curve with closest effective stress

2. On $\varepsilon-p$ curve:

a. Perform Sallfors method

3. On $W$ - $p$ curve:

a. Perform work (Becker et al.) method

b. Perform Wang and Frost (2004) method

4. On $\log (1+e)-\log p, \ln (1+e)-\ln p$ curves:

a. Perform bilogarithmic methods (3)

5. On $D$ - $p$ curve:

a. Perform Janbu (1963) method 


\subsection{SUMMARY}

The numerical methods described in this section are of two types:

1) Identifying key points (point of maximum curvature and inflection point) on the $e$-logp curve. These methods are newly introduced in this study.

2) Using the equations of straight lines to both mathematically plot the lines required to perform the graphical methods and calculate the intersection of those equations to determine the preconsolidation pressure. This approach was introduced by Boone (2010) but is elaborated upon in this study.

It would be desirable to compare the point of maximum curvature obtained by this numerical method with that which would be identified visually by an engineer. However, given the subjective nature of locating this point visually, such a comparison would be most effective if many engineers were asked to locate the point of maximum curvature when given the same data set, and then comparing their results with that obtained by the numerical method. Such an investigation is outside the scope of this study. Rather, this method of locating maximum curvature is to be evaluated based on ease of use and objectivity.

It should be emphasized that the approach of plotting straight lines by the use of equations does not in any way change the fact that the procedures to determine preconsolidation pressure are graphical in nature. The difference lies in the degree of control that the engineer has in plotting the straight lines, and in the fact that preconsolidation pressure can now be calculated rather than interpreted visually. Although this can technically be done without plotting the equations, it is vital to still draw the lines over the test curve in order to visually check that the interpretations of point of maximum curvature, inflection point, slope of the curve at various points and preconsolidation pressure are valid. 


\section{EVALUATIONS OF DIFFERENT METHODS BASED ON CRS TEST DATA}

\subsection{INTRODUCTION}

Ten graphical methods to estimate preconsolidation pressure were applied to the results of 11 CRS tests on Champlain Sea clay. The results of this analysis will be presented and discussed. First, overall trends will be presented. The results of each method will then be discussed individually. Finally, the numerical methods that were used to analyze the data will be evaluated.

\subsection{Method for Evaluation of Performance of Different Methods}

\subsubsection{Method for Evaluation using Reloading Stages}

Of the 11 CRS tests that were performed, 9 had unloading/reloading cycles that could be used to evaluate the accuracy of the graphical methods. As such, the graphical methods were applied to the initial loading stages of all 11 tests, as well as to the reloading stages of 9 of the tests. When performing the graphical methods on the reloading stages, the maximum past pressure was known. This was the effective stress at the end of the initial loading stage, prior to unloading. An example is shown in Figure 5-1. The maximum past pressure was not obtained graphically, but rather based on the effective stress calculated for the final reading during the initial loading stage.

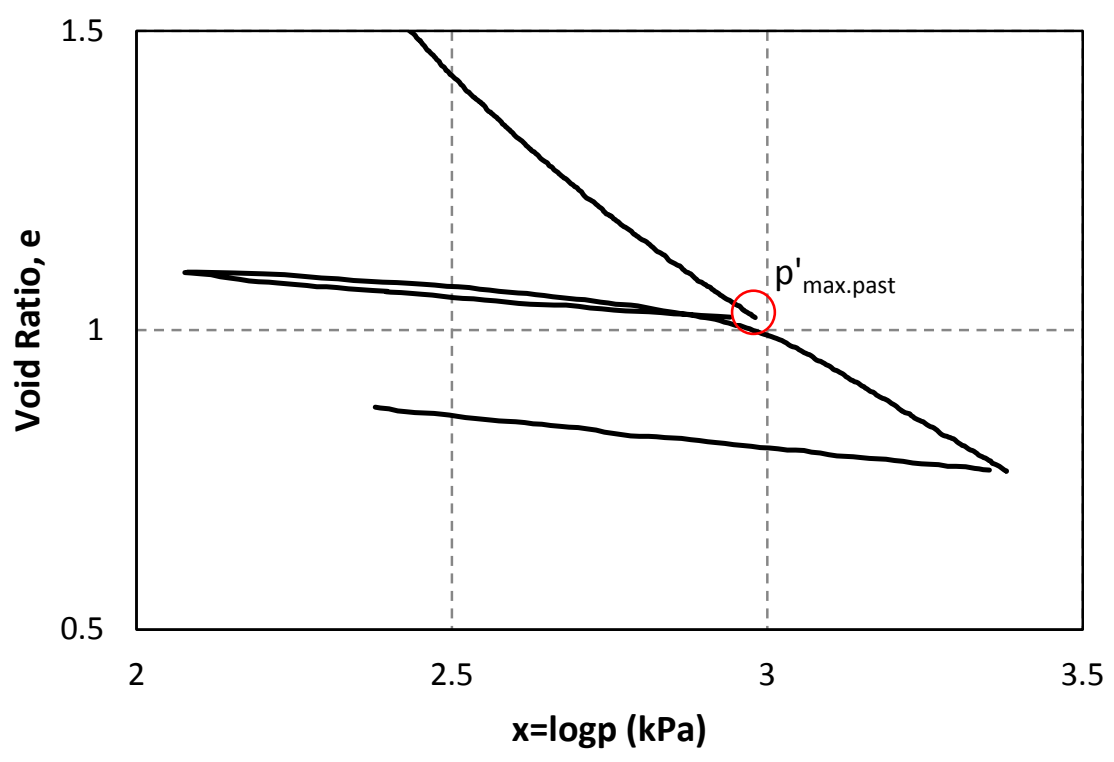

Figure 5-1: Example of obtaining known maximum past pressure 
For illustration purposes, the result of the $e$-logp bilinear method when performed on the reloading stage shown in the preceding figure is shown in Figure 5-2.

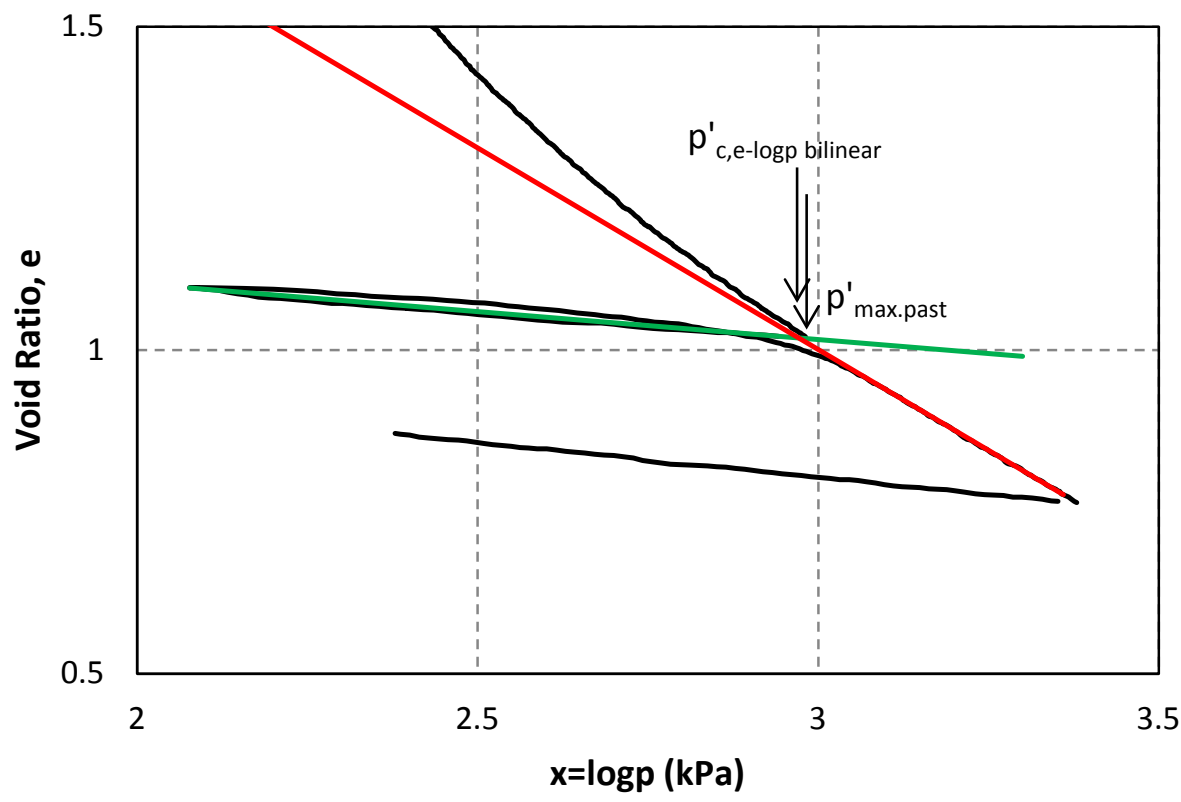

Figure 5-2: Demonstration of comparing known to estimated preconsolidation pressure

The result of each method was compared to this known maximum past pressure in order to obtain the error associated with each method. The average error was calculated using the formula:

$$
\text { Average Absolute Error }(\%)=\frac{1}{n}\left(\sum_{i=1}^{n}\left|\frac{p_{c}^{\prime}-p_{\text {max.past }}^{\prime}}{p_{\text {max.past }}^{\prime}}\right|\right) * 100
$$

For each graphical method, a plot was created with the preconsolidation pressure as defined by that method on the vertical axis and the known maximum past pressure on the horizontal axis. Each point corresponds to one test. Points that fall above the 1:1 ratio line show that the preconsolidation pressure found using a given method is higher than the known maximum past pressure. Points that fall below this line show that it is lower.

Linear regression is then performed to quantify the accuracy and precision of the method. The closeness of fit of a line is typically measured using the regression coefficient $\mathrm{R}^{2}$. The use of $\mathrm{R}^{2}$ when employed in this way measures the precision of a method, but not necessarily its accuracy. The accuracy of a method can be evaluated based on the coefficients of the regression equation. A slope of 1 and an intercept of 0 would indicate that the results obtained by any given method are identical to the values against which they are being compared. A high $\mathrm{R}^{2}$ with a slope other than 1 or intercept other than 0 would indicate that two methods give different results but are 
strongly correlated. This would suggest that some correction factor could translate a result from one method to the other.

An example of such a plot is shown in Figure 5-3. The solid black line represents a ratio of 1:1 and the red line shows the line of best fit obtained by linear regression. The equation of the red line is shown on the figure.

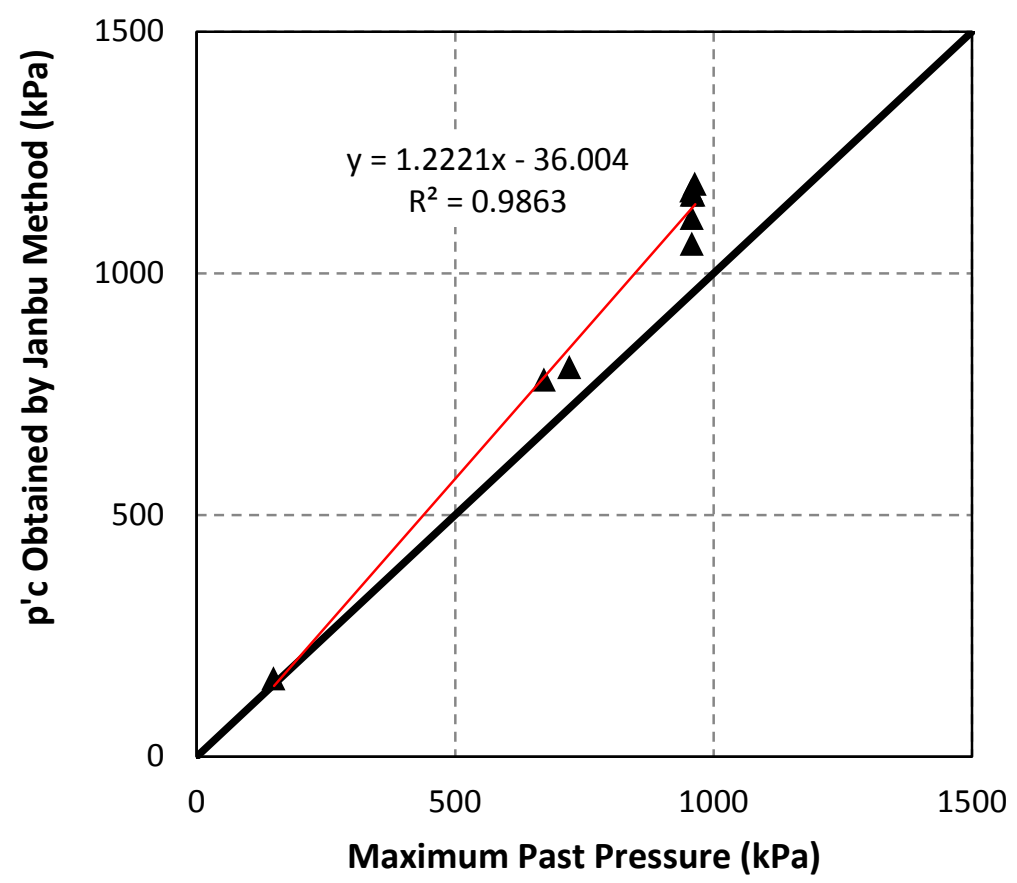

Figure 5-3: Example of plotting results obtained by a graphical method against the known maximum pressures for reloading stages

\subsubsection{Method for Evaluation using Initial Loading Stages}

For the initial loading stages, no known values are available against which to compare the preconsolidation pressures obtained using the different methods. Thus, some method must be selected as a baseline against which to compare each of the others. Two methods are chosen for this purpose: the $\ln (1+e)$-lnp bilogarithmic method and the work method. The justification for using the work method as a point of comparison is its frequent use in engineering practice, as well as the claim that it is the most theoretically sound of all the methods (Grozic, Lunne, \& Pande, 2003). The bilogarithmic method is also used due to its excellent performance in the reloading stages.

The results were also evaluated based on the percent difference between each method and the baseline method. These values cannot be considered as errors, as the methods are being compared against an arbitrary baseline rather than a known value. 
The percent differences are calculated as:

$$
\begin{aligned}
\text { Percent Difference }_{W o r k} & =\left|\frac{p_{c}^{\prime}-p_{c, W o r k}^{\prime}}{p_{c, \text { Work }}^{\prime}}\right| * 100 \% \\
\text { Percent Difference }_{\ln (1+e)-\ln p} & =\left|\frac{p_{c}^{\prime}-p_{c, \ln (1+e)-\ln p}^{\prime}}{p_{c, \ln (1+e)-\ln p}^{\prime}}\right| * 100 \%
\end{aligned}
$$

\subsubsection{Overview of Results from Reloading Stages}

For each graphical method, the results were plotted against the known maximum past pressures and the linear regression equations were obtained. The results of this analysis are summarized in Table 5-1.

Table 5-1: Regression equations for results of each method with known maximum past pressure in reloading cases

\begin{tabular}{|c|c|c|}
\hline Method & Regression Equation & $\mathbf{R}^{\wedge} \mathbf{2}$ \\
\hline loglog & $0.978^{*} \mathrm{x}+13.985$ & 0.9974 \\
\hline Inln & $0.978^{*} \mathrm{x}+13.985$ & 0.9974 \\
\hline e-logp bilinear & $0.936^{*} \mathrm{x}+25.728$ & 0.9947 \\
\hline Casagrande & $1.113^{*} \mathrm{x}-11.649$ & 0.9944 \\
\hline Nagaraj et al. & $1.195^{*} \mathrm{x}-12.333$ & 0.9901 \\
\hline Pacheco Silva & $0.897^{*} \mathrm{x}+44.743$ & 0.9869 \\
\hline Janbu & $1.222^{*} \mathrm{x}-36.004$ & 0.9863 \\
\hline Becker et al. & $0.887^{*} \mathrm{x}+42.664$ & 0.9859 \\
\hline Sallfors & $1.089^{*} \mathrm{x}-14.642$ & 0.9746 \\
\hline Wang and Frost & $0.926^{*} \mathrm{x}+32.143$ & 0.9770 \\
\hline Peck & $0.661^{*} \mathrm{x}+101.570$ & 0.9016 \\
\hline
\end{tabular}


The average error for each method from the reloading tests is summarized in Table 5-2

Table 5-2: Average absolute percent error of each method for the reloading tests

\begin{tabular}{|c|c|}
\hline Method & $\begin{array}{c}\text { Average } \\
\text { Absolute Error } \\
\text { (\%) }\end{array}$ \\
\hline loglog & 1.415 \\
\hline lnln & 1.415 \\
\hline e-logp bilinear & 3.699 \\
\hline Wang and Frost & 4.701 \\
\hline Pacheco Silva & 5.541 \\
\hline Becker et al. & 5.945 \\
\hline Casagrande & 9.314 \\
\hline Janbu & 16.597 \\
\hline Sallfors & 17.230 \\
\hline Nagaraj et al. & 17.651 \\
\hline Peck & 19.064 \\
\hline
\end{tabular}

A number of observations can be made from the above data. First, the two variations of the bilogarithmic method yielded identical results. Second, the bilogarithmic methods yielded the lowest average error of all the methods. Third, the methods that define preconsolidation as the intersection of pre-yield and post-yield lines (bilogarithmic, $e$-logp bilinear and Work methods) gave similar but non-identical results. Fourth, the Wang and Frost method, which was introduced as an improvement on the work method, yielded a lower error than the work method. Finally, the three methods that yielded the largest errors were the Peck method (underpredicted), the Nagaraj et al. method (overpredicted), and the Janbu method (overpredicted).

Alternatively to the method shown in Figure 5-3, the axes of the plot can be reversed. The resulting regression equation can then be used as a correction to obtain an estimated true preconsolidation pressure based on the results of each method. An example of this procedure is shown in Figure 5-4. The uncorrected preconsolidation pressure is shown in black and the corrected preconsolidation pressure is shown in red. It can be seen that the corrected preconsolidation data points fall much closer to the 1:1 ratio line. Furthermore, the regression line of the corrected data points coincides with that line. Although the error of some individual tests may increase as a result of being shifted to the opposite side of the 1:1 line, the average error of the method decreases. 


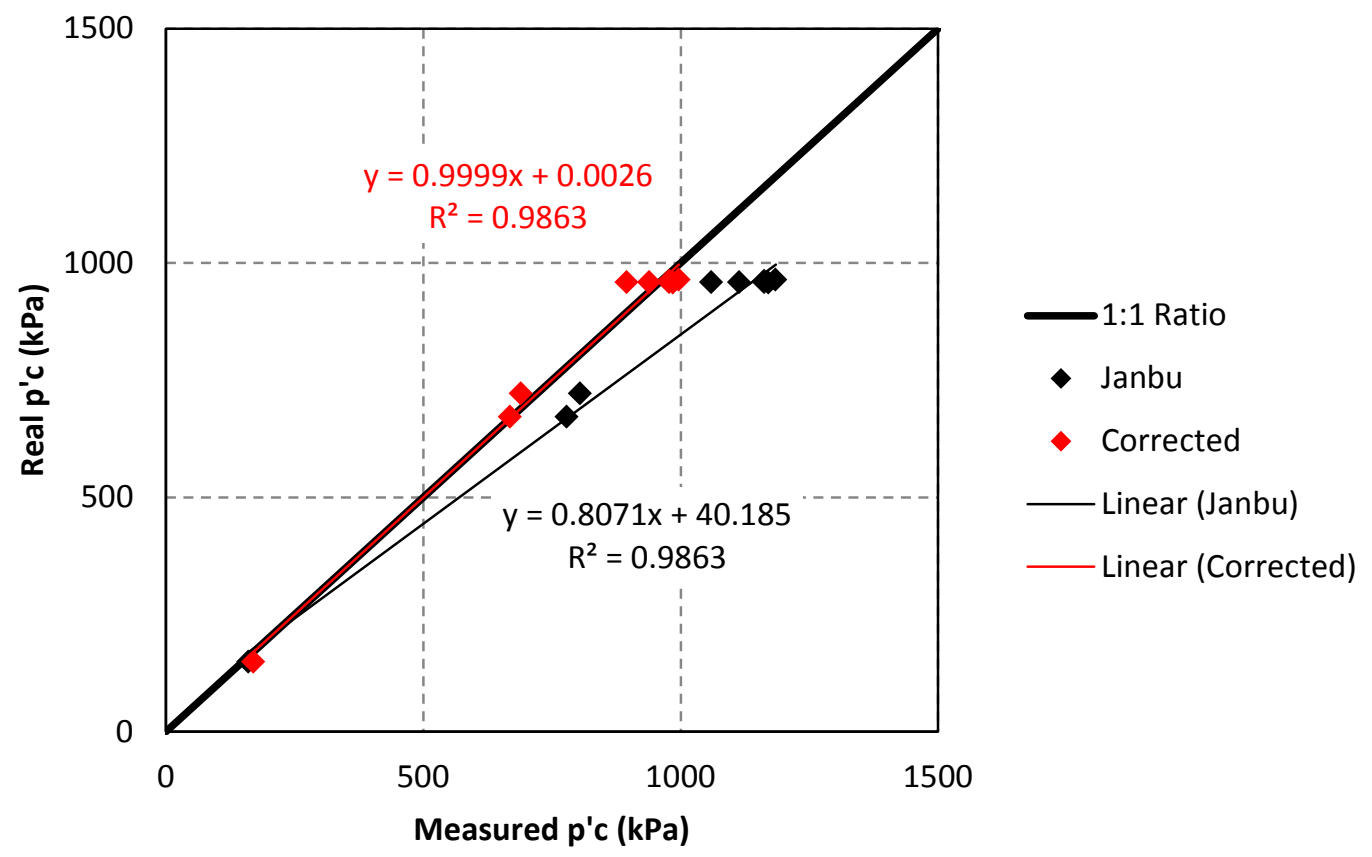

Figure 5-4: Comparison of uncorrected and corrected preconsolidation pressures in reloading tests for the Janbu method

The correction equation obtained for each method by the procedure demonstrated in Figure 5-4 is shown in 
Table 5-3. After applying these corrections, a new average absolute error is obtained for each method. These results are also summarized in 
Table 5-3. In nearly all cases, the average error decreased as a result of applying the correction. Interestingly, the average error for the bilogarithmic methods went slightly up. 
Table 5-3: Correction equations and resulting improvements to average absolute error for each method

\begin{tabular}{|c|c|c|c|}
\hline Method & Correction Equation & $\begin{array}{c}\text { Corrected } \\
\text { Average } \\
\text { Absolute Error } \\
\mathbf{( \% )}\end{array}$ & $\begin{array}{c}\text { Change in Average } \\
\text { Absolute Error } \\
\text { due to Correction } \\
\mathbf{( \% )}\end{array}$ \\
\hline $\operatorname{loglog}$ & $1.01 *^{*} \mathrm{x}-9.256$ & 1.686 & +0.271 \\
\hline $\operatorname{lnln}$ & $1.01 *^{*} \mathrm{x}-9.256$ & 1.686 & +0.271 \\
\hline e-logp bilinear & $1.059 * \mathrm{x}-19.993$ & 2.612 & -1.087 \\
\hline Wang and Frost & $1.056^{*} \mathrm{x}-15.265$ & 4.110 & -0.591 \\
\hline Pacheco Silva & $1.10 *^{*} \mathrm{x}-38.592$ & 3.873 & -1.668 \\
\hline Becker et al. & $1.113^{*} \mathrm{x}-36.920$ & 4.195 & -1.750 \\
\hline Casagrande & $0.894 * \mathrm{x}+14.955$ & 2.204 & -7.110 \\
\hline Janbu & $0.807 * \mathrm{x}+40.185$ & 4.135 & -12.462 \\
\hline Sallfors & $1.089 * \mathrm{x}-9.436$ & 2.199 & -15.032 \\
\hline Nagaraj et al. & $0.828^{*} \mathrm{x}+18.258$ & 3.126 & -14.525 \\
\hline Peck & $1.364 * \mathrm{x}-58.725$ & 8.644 & -10.420 \\
\hline
\end{tabular}

\subsubsection{Overview of Results from Initial Loading Stages}

The results of the initial loading tests were evaluated based on the percent difference between each method and the baseline method. These differences are presented in 
Table 5-4, where comparisons against both the bilogarithmic and work methods are presented. These values are not errors, as the methods are being compared against an arbitrary baseline rather than a known value.

After calculating the percent difference of each test for each method, the average absolute percent difference for each method was calculated. These averages are summarized 
Table 5-4 below. 
Table 5-4: Comparison of percentage differences between work method and $\ln (1+e)-\ln p$ bilogarithmic method for initial loading

\begin{tabular}{|c|c|c|}
\hline Method & $\begin{array}{c}\text { Average Absolute } \\
\text { In(1+e)-Inp Error (\%) }\end{array}$ & $\begin{array}{c}\text { Average Absolute } \\
\text { Work Error (\%) }\end{array}$ \\
\hline loglog & 0 & 1.605398 \\
\hline Inln & 0 & 1.605398 \\
\hline e-logp bilinear & 0.94199 & 2.167393 \\
\hline Becker et al. & 1.633173 & 0 \\
\hline Wang and Frost & 2.326788 & 1.967909 \\
\hline Pacheco Silva & 4.016716 & 3.057638 \\
\hline Casagrande & 7.91391 & 6.758151 \\
\hline Peck & 10.35774 & 11.28972 \\
\hline Sallfors & 11.387 & 12.262 \\
\hline Janbu & 20.65611 & 19.39326 \\
\hline Nagaraj et al. & 58.6809 & 56.87178 \\
\hline
\end{tabular}

Once again, a number of observations can be made from the above table. First, the three methods that gave the largest errors in the reloading tests (Nagaraj et al., Janbu and Peck) also give the largest percent differences in the initial loading tests. Similarly, the Casagrande method, which was relatively inaccurate in the reloading tests, performed similarly in comparison to the other methods in the initial loading tests. Second, the percent differences associated with the six remaining methods were very similar, just as their errors were very similar for the reloading tests. Finally, the two variations of the bilogarithmic method once again gave identical results. All of these observations suggest that the results obtained in the reloading tests are also applicable in the initial loading tests.

The values obtained by each method can be plotted to see the increase in preconsolidation pressure with depth. Examples of this are shown for two methods in Figure 5-5 and Figure 5-6. The corrections described in 
Table 5-3 are applied to these results and the resulting corrected preconsolidation pressures are also shown in the figures. It can be seen that all values of preconsolidation pressure are either unchanged or decreased in these examples.

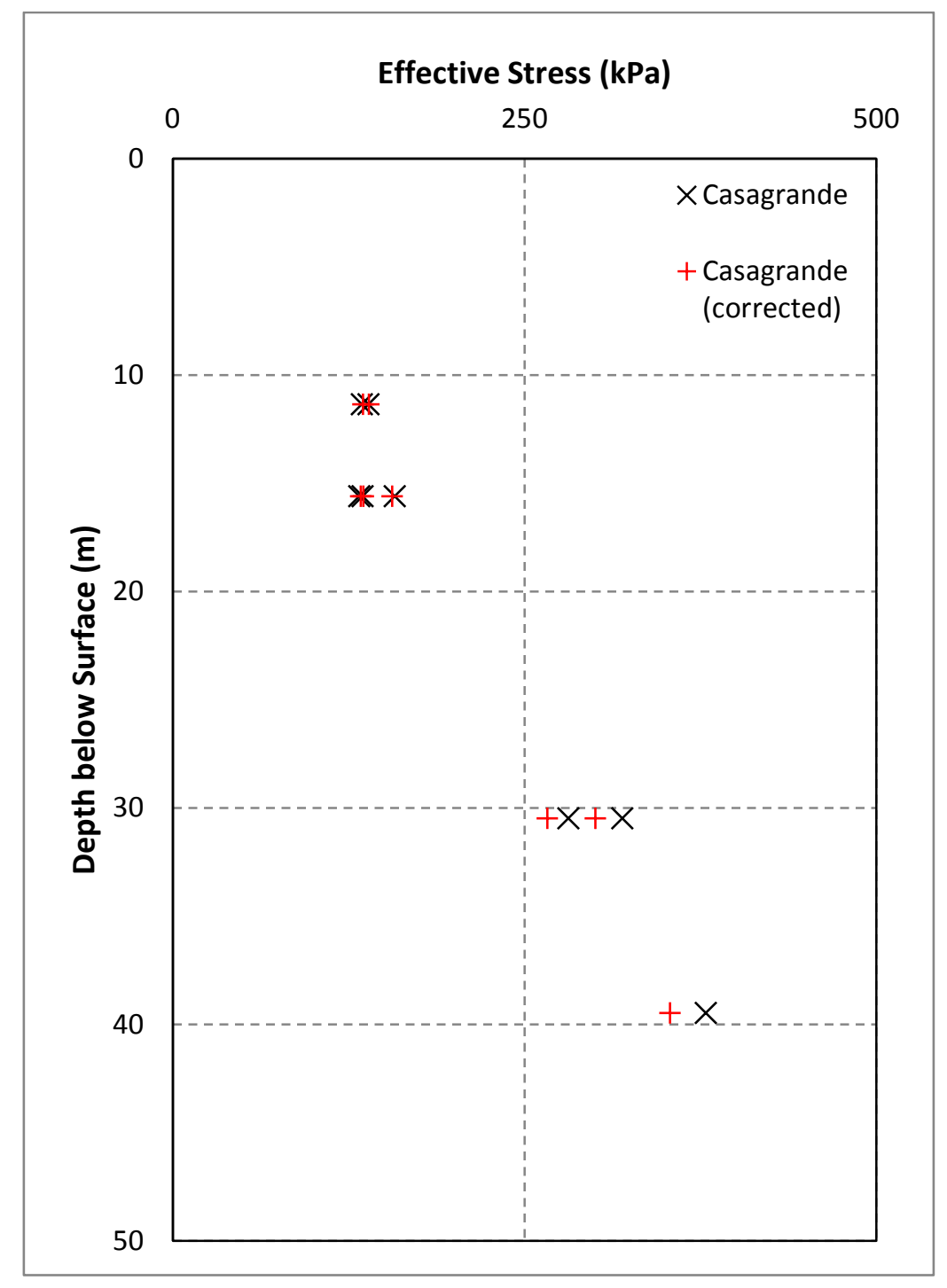

Figure 5-5: Preconsolidation pressure vs. depth with and without correction according to Casagrande method 


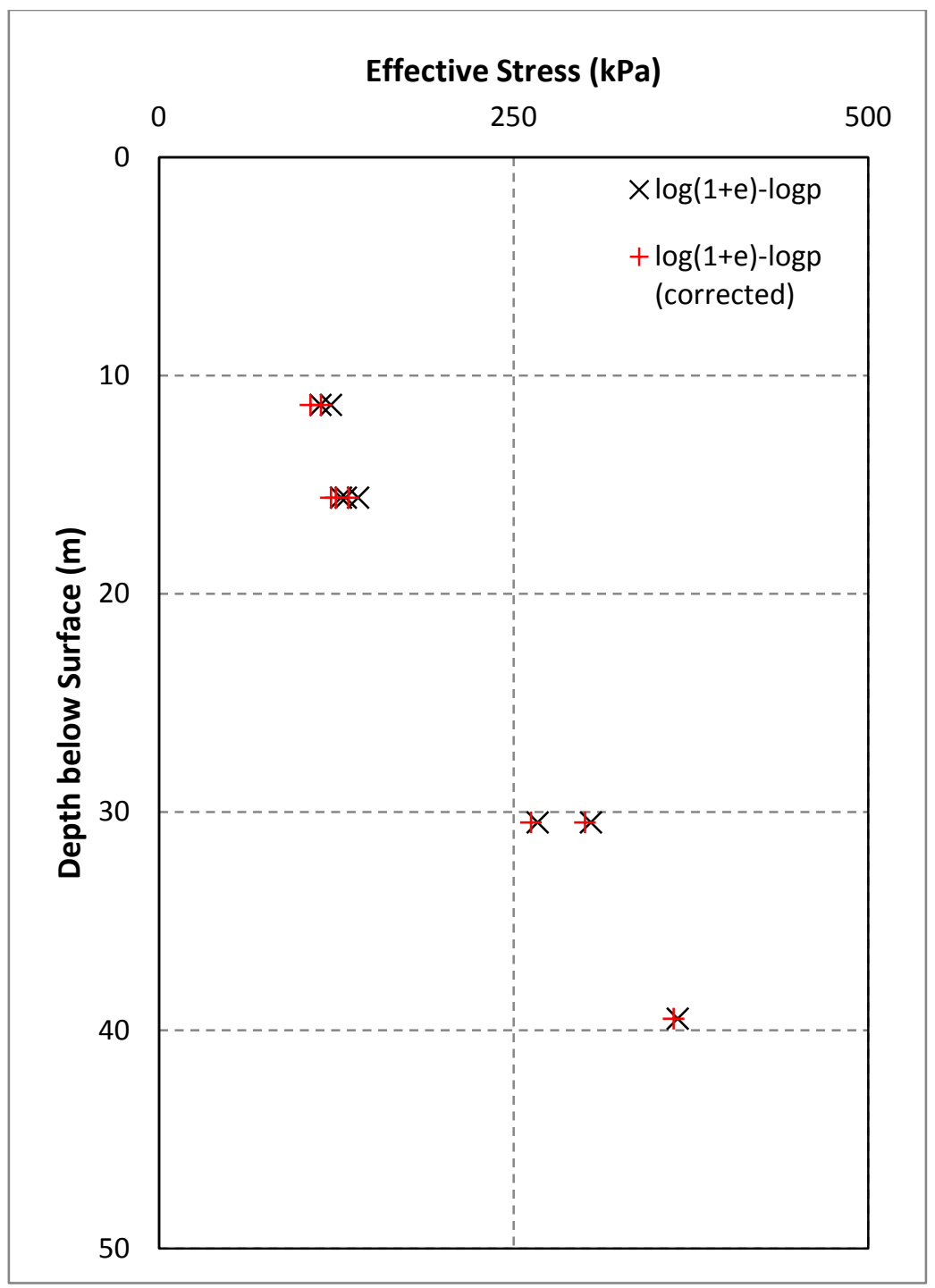

Figure 5-6: Preconsolidation pressure vs. depth with and without correction according to $\log (1+e)-\log p$ method

\subsubsection{Typical CRS Test Results}

The typical "S-shape" of sensitive clay was found for all of the 11 tests in this study. This could be seen in the $e-\log p, \log (1+e)-\log p, \ln (1+e)-\ln p$ and $W$-p plots. These plots are shown for a typical test in the following figures. These figures have been trimmed to remove the effect of seating error early in the test. 


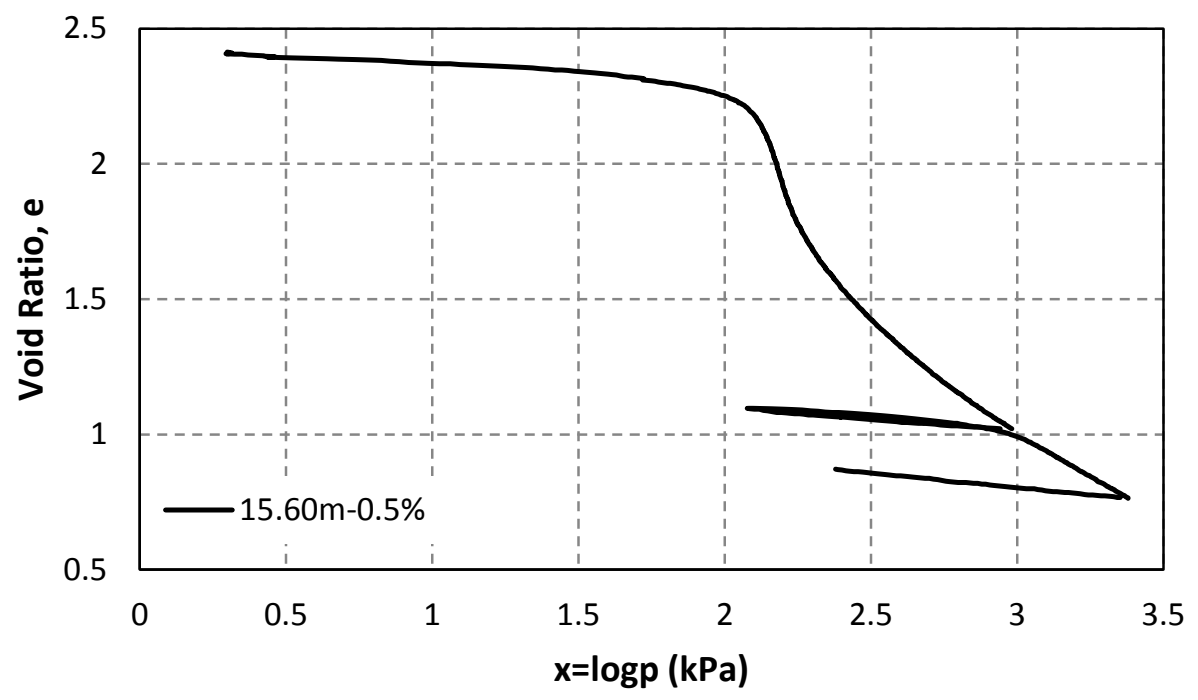

Figure 5-7: e-logp plot for a typical test

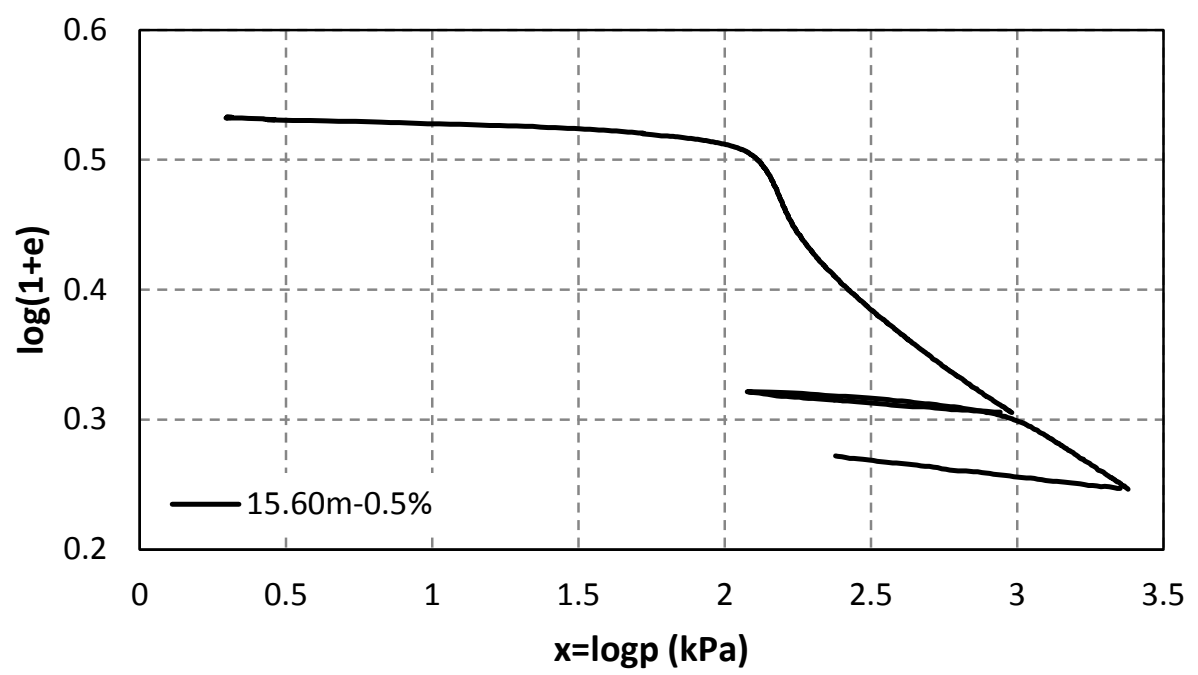

Figure 5-8: $\log (1+e)-\log p$ plot for a typical test 


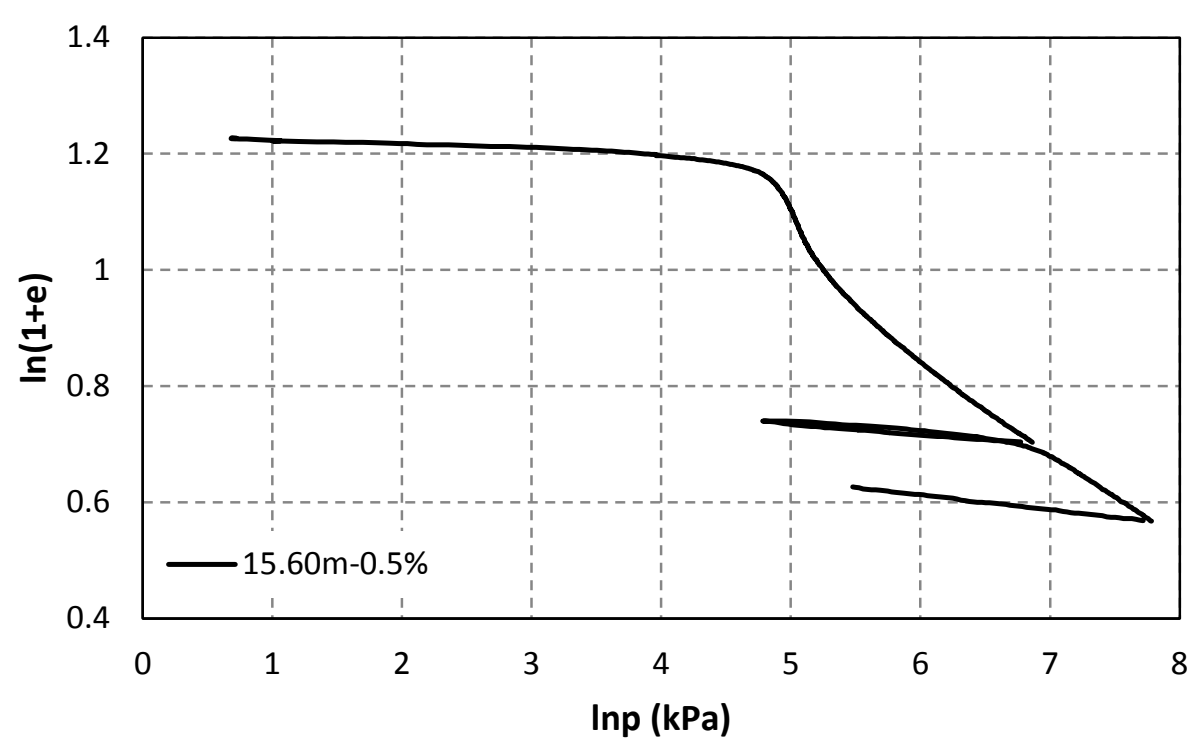

Figure 5-9: $\ln (1+e)-\ln p$ plot for a typical test

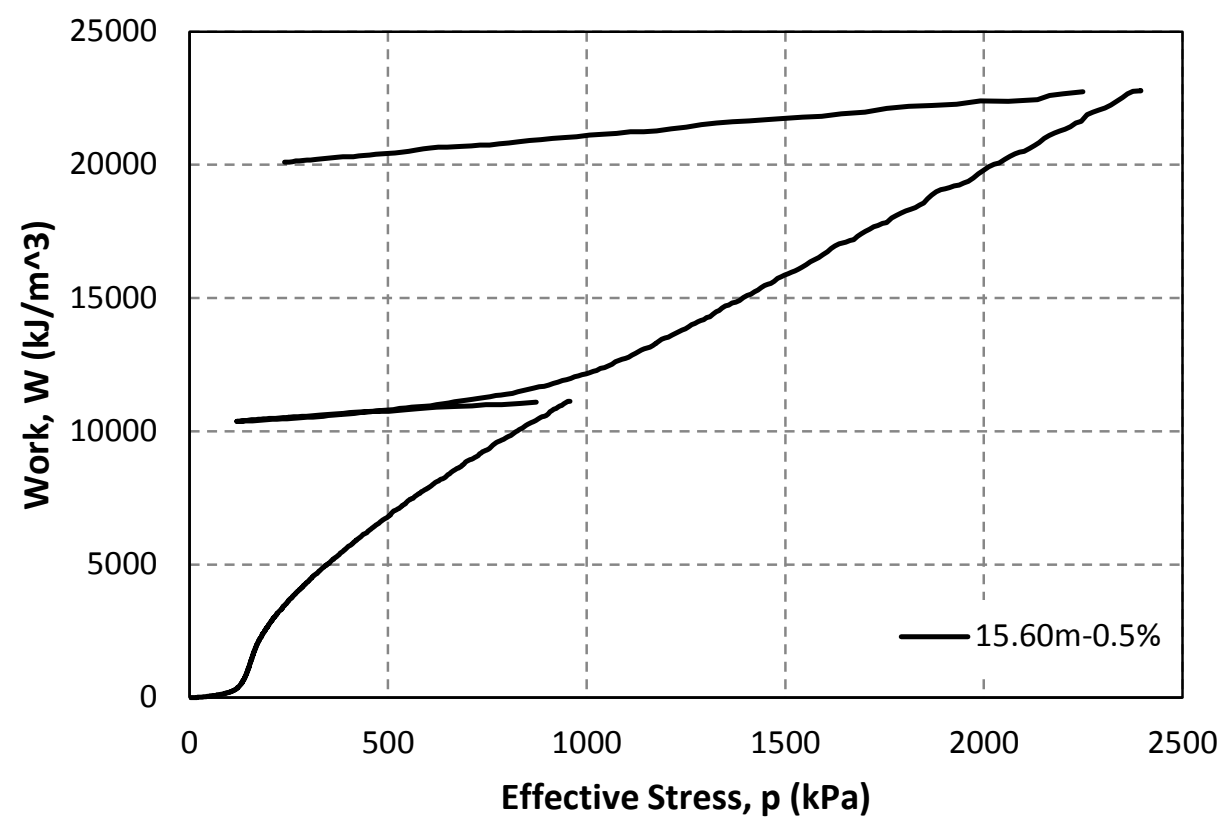

Figure 5-10: $W-p$ plot for a typical test

The "S-shape" was not visible in the $D-p$ plot used in the Janbu method. This plot is shown for the same test in the figure below. The peak in the early part of the test is the continued result of seating effects rather than preconsolidation. The data for this curve has been trimmed to the same range as the preceding curves. Furthermore, median smoothing has been applied to the constrained modulus data in order to reduce the amount of scatter in the plot. 


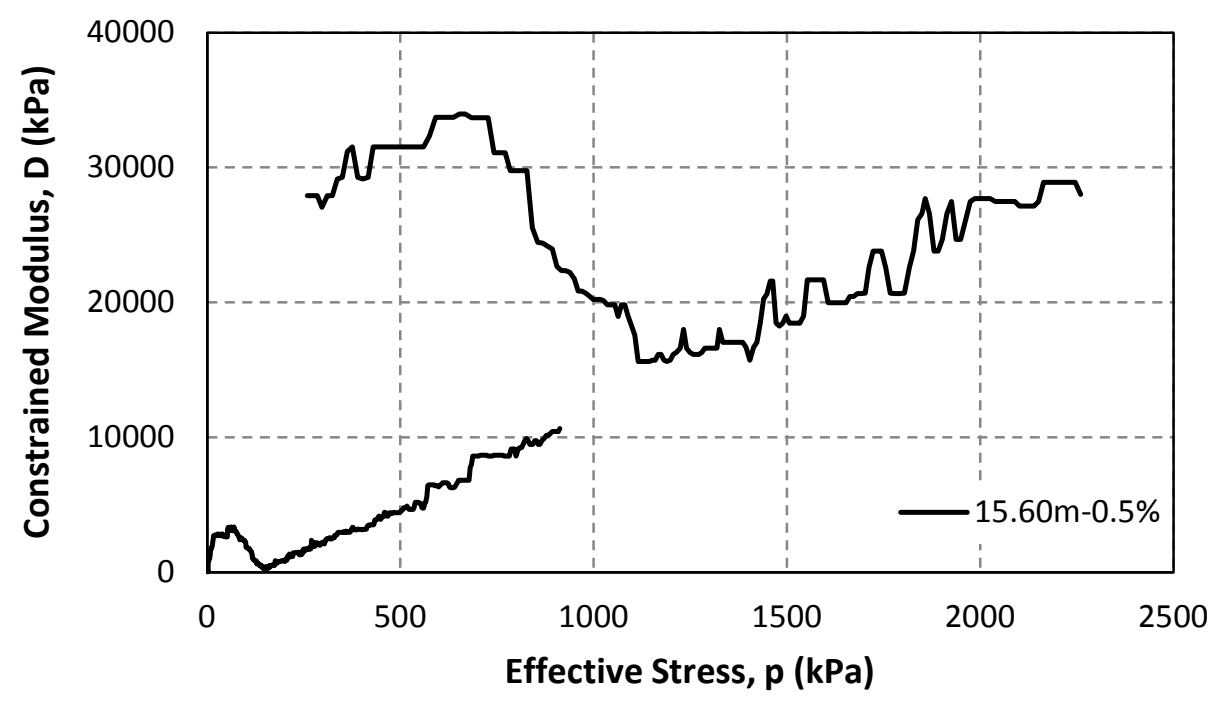

Figure 5-11: $D-p$ plot for a typical test

\subsection{Performance of Each Method}

In the following sections, the results obtained using each of the methods for determining preconsolidation pressure will be discussed individually. Each method was performed according to the numerical procedures described in Section 4.

\subsubsection{Casagrande Method}

When compared against the known maximum past pressures in the reloading tests, the Casagrande method gave the fifth largest absolute value of percent error of the eleven methods, indicating that it is relatively inaccurate.

In all tests, the point of maximum curvature was determined assuming that the ratio of e to logp is $1: 1$. This is not necessarily the ratio that would be selected if the method is applied purely graphically, as different users might choose to plot the e-logp data on plots with different amounts of exaggeration of a particular axis. The influence of changing this ratio should be investigated in a future study.

The results support the conclusion of Grozic et al. (2003) that the Casagrande method tends to overpredict the preconsolidation pressure based on known maximum past pressures and that it is less accurate than other methods. 


\subsubsection{Peck Method}

The Peck method was expected to give the lowest result. Several methods define the preconsolidation pressure as occurring somewhere along the virgin compression line. In the case of sensitive clay, this is the Zone II line, which is extended upward. The Peck method defines preconsolidation pressure the farthest up and therefore the farthest to the left of all the methods. Thus, the Peck method is expected to yield the lowest preconsolidation pressure.

This was indeed found to be the result. In fact, the Peck method was by far the method that most underestimated the preconsolidation pressure, and gave the highest error in the reloading tests (19.06\%). This is due to the fact that the Peck method is specific to sensitive clays. During the reloading stage, the cementation bonds that are present in sensitive clays have already been broken, so the slope of the pre-yield line (or recompression line) is much steeper. This causes a larger distance over which the post-yield line must be extended, thus causing the Peck method to give a much lower value. The same phenomenon would presumably contribute to the Peck method underestimating preconsolidation pressure for highly disturbed samples.

The Peck method was also found to show the largest amount of scatter of all the test methods. For the reloading tests, the plot of preconsolidation pressure obtained by the Peck method against known maximum past pressure gave an $\mathrm{R}^{2}$ of 0.902 . This is by far the lowest of all the methods investigated. For comparison, the next lowest $\mathrm{R}^{2}$ was obtained for the Wang and Frost method, which gave a significantly higher value of 0.977 .

A huge difficulty in performing the Peck method during initial loading tests comes from the effects of seating error. In tests where seating error occurred, it was necessary to trim the data to remove seating error from the $e$-logp plot. However, in some cases, it was not clear what point should be used as the first point on the consolidation curve. This has a large influence on the Peck method, as it would affect the position of the void ratio line relative to the rest of the test curve. If the void ratio line is higher, then a lower result is obtained by the Peck method.

\subsubsection{Pacheco Silva Method}

For the reloading tests, the Pacheco Silva method was in the bottom half of the results studied based on both $\mathrm{R}^{2}(0.987)$ and percent error (-3.76\%). However, these results are still fairly good, given the close similarity of the six most accurate methods.

The Pacheco Silva method performed better than the work method (-5.11\%). This is an interesting result, as the Pacheco Silva method appears to be developed based on graphical observations only, whereas the work method is claimed to be based on theoretical principles.

A contrary result was found to that of Umar and Sadrekarimi (2016). They found that the bilogarithmic, work, Pacheco Silva and Casagrande methods all slightly overestimated the 
preconsolidation pressure for reloading tests. In the present study, it was found that all these methods except for the Casagrande method slightly underestimated the preconsolidation pressure in reloading tests.

\subsubsection{Nagaraj et al. Method}

The procedure that was used to perform the method of Nagaraj et al. was slightly modified from what is proposed by the original authors of that method, due to the lack of a means to create a resedimented soil sample. The modified method is based on the observation by Nagaraj et al. (1990) that the line that is perpendicular to the resedimented line and that passes through the point of maximum curvature will also be perpendicular to the $e$-logp curve at that point. Thus, rather than obtaining a resedimented line on which to draw a perpendicular line, a line perpendicular to the $e$-logp curve at the point of maximum curvature was used instead.

For the initial loading stages, this procedure gave very scattered results. Its $\mathrm{R}^{2}$ value was 0.807 when compared to the work method. The next weakest correlation was found with the Janbu method, which was $\mathrm{R}^{2}=0.953$. The sharp curvature of the e-logp curve at the point of maximum curvature likely has an influence on this result. While the selection of point of maximum curvature has a relatively small influence on the result obtained by the Casagrande method, it has an enormous influence on the result obtained by this modified Nagaraj et al. method.

This can be attributed to two factors. The first is the fact that the slope of the $e$-logp curve changes very rapidly around the point of maximum curvature due to the sharp drop of the "Sshape”. Thus, if the selected point is shifted just slightly to one side or another, the determination of preconsolidation pressure will be drastically different. In the figure below, although the selected points that could represent maximum curvature are very close, the resulting values of preconsolidation pressure are $320 \mathrm{kPa}$ and $798 \mathrm{kPa}$ (a factor of more than 2). This difference is exaggerated by the fact that effective stress is plotted on a logarithmic scale.

The second factor that influences this high degree of inconsistency is the fluctuation of the stress sensor. The slope of the curve obtained at the point of maximum curvature does not necessarily accurately reflect the average slope of the curve at that point. To address this, a different type of data smoothing might be required.

For the reloading tests, the Nagaraj et al. method was the method that most overpredicted the preconsolidation pressure, with an average percent error of $17.65 \%$. Additionally, the degree of scatter was considerably lower for the reloading tests, as the $\mathrm{R}^{2}$ value was 0.990 . Five other methods had lower regression coefficients. This can be explained by the fact that the curvature during the reloading tests is considerably flatter during reloading tests.

These results indicate that the preconsolidation pressure obtained by the Nagaraj et al. method do not correlate with those obtained by the other methods. It is possible that the results obtained by 
the Nagaraj et al. method do, in fact represent the preconsolidation pressure in the field, and that the large discrepancy between the results of this method and those obtained by the other methods are due to sample disturbance. If this is the case, this is evidence that results obtained by the other methods must be corrected for sample disturbance. Unfortunately, it is not possible to accurately determine the field preconsolidation pressure for the site from where the samples originate.

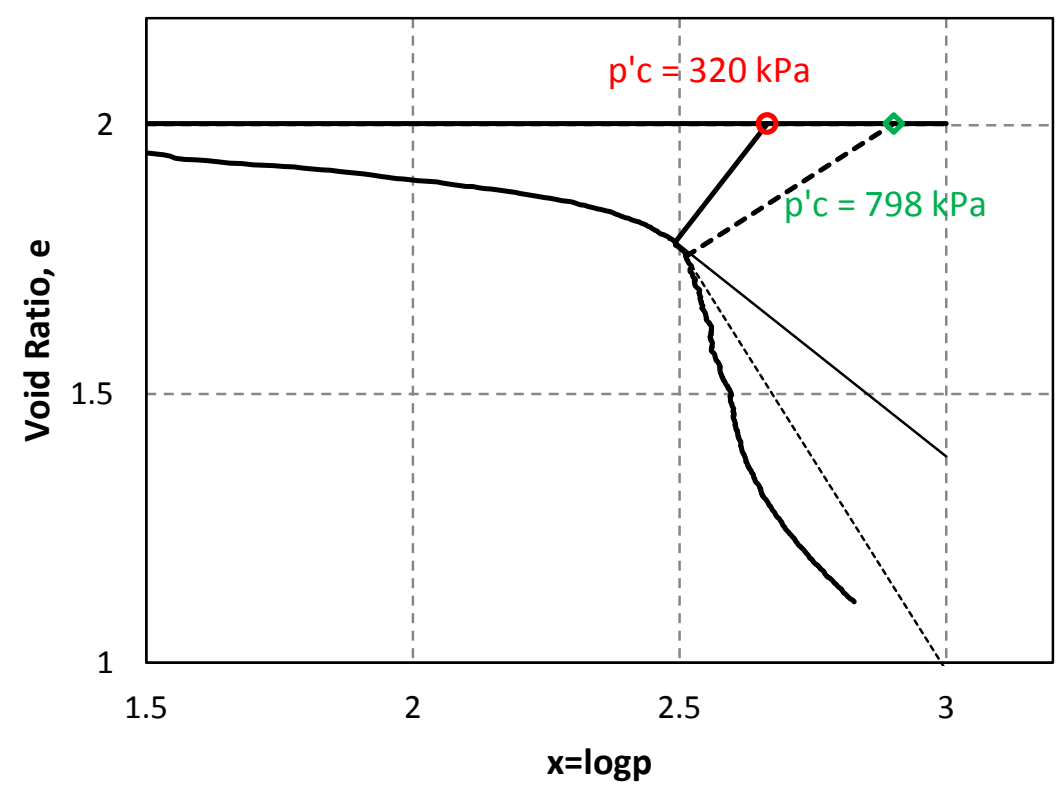

Figure 5-12: Effect of selection of point of maximum curvature on p'c obtained by method of Nagaraj et al. (1990)

\subsubsection{Work Method}

Although the work method proposed by Becker et al. (1987) is widely used in the industry, it did not perform as well as the other methods that similarly define the preconsolidation pressure as the intersection between pre-yield and post-yield lines. The average error for the reloading tests was $-5.11 \%$, compared with only $-0.49 \%$ for the bilogarithmic methods and $-2.87 \%$ for the $e$ logp bilinear method. The correlation with known maximum past pressures was also lower for the work method (0.986) than for those other two methods (0.997 and 0.993, respectively).

It is interesting that the work, bilogarithmic and $e$-logp bilinear methods give similar but nonidentical results, given their similar definitions of preconsolidation pressure. A possible explanation for this result is that the bilogarithmic methods do, in fact, give straighter lines in the pre-yield and post-yield regions of the test curve. Nevertheless, the results of the work method were closer to those of the $e$-logp bilinear method and the bilogarithmic method than they were to any of the other methods. Further discussion is provided in Section 5.3.7. 


\subsubsection{Wang and Frost Method}

The Wang and Frost method resulted in a lower average percent error (-2.91\%) than the work method (-5.11\%). This could possibly provide evidence for the claim by Wang and Frost (2004) that the basis of their method in dissipated strain energy makes their method more accurate than the Becker et al. method, which is based on total strain energy. However, the correlation with known maximum past pressure is weaker for the Wang and Frost method (0.977) than for the work method (0.986), so this result is not necessarily conclusive. Furthermore, this result is opposite to what was found by Umar and Sadrekarimi (2016), who found that the work method gave lower average error.

For the initial loading tests, the Wang and Frost method was found to give results slightly lower than the work method, with the average percent difference being $-1.34 \%$. This was the second lowest value of percent difference compared to the work method, with only the bilogarithmic methods giving a lower value. This similarity between the Wang and Frost method and the work method is logical, given that the former is only a very slight modification of the other.

It is not clear whether or not the Wang and Frost method is suitable for sensitive clays, as it is based on the assumption that the recompression line is a truer representation of the recompression index. However, in the case of sensitive clays, the presence of cementation bonds causes the curve to have a much shallower slope prior to structural breakdown.

\subsubsection{Bilogarithmic Methods}

The bilogarithmic methods gave the most accurate results for the reloading tests, with an average error of $-0.49 \%$ (lowest of all the methods) and an $\mathrm{R}^{2}$ of 0.997 (highest of all the methods).

The two variations of the bilogarithmic method, namely $\log (1+e)-\log p$ and $\ln (1+e)-\ln p$, gave identical results for all tests. This is reflected by the fact that the regression of one bilogarithmic method against the other gave a result of $\mathrm{R}^{2}=1$ and slope $=1$.

It was also found that the slopes of the pre-yield and post-yield lines were identical for the two methods for all tests. For example, the slope of the recompression line for one set of bilogarithmic axes was identical to the slope of the recompression line for the other. The following relation can help explain this result:

$$
\frac{\Delta \ln (1+\mathrm{e})}{\Delta \ln p}=\frac{2.3 * \Delta \log (1+e)}{2.3 * \Delta \log p}=\frac{\Delta \log (1+e)}{\Delta \log p}
$$


A contributing factor in these results was the fact that the same points were used to calculate the slopes of the pre-yield and post-yield lines regardless of the method being used. This result supports the claim that the two methods are mathematically equivalent.

Past researchers have not found that the bilogarithmic methods give identical results. For example, Umar and Sadrekarimi (2016) found that the different bilogarithmic methods gave very similar but non-identical results. The slight difference in their results probably results from performing the methods graphically, whereas in the present study, the same points from the CRS test were selected to draw the pre-yield and post-yield lines.

For the reloading tests, the slope of the recompression line was obtained by connecting the two points where the unloading and reloading curves intersect. This line typically happens to connect with the point of maximum past pressure. Thus, it is expected that using the unloading stage as the pre-yield line in the analysis will provide a very accurate representation of the preconsolidation pressure for a reloading type test, depending on the slope of the post-yield line.

It was observed that, in some of the tests, the consolidation curve during reloading totally resumed the path it had been following before unloading commenced after exceeding the maximum past pressure. In other tests, however, the path of the curve during reloading was slightly offset from what it had been following before unloading. It is suspected that this phenomenon is responsible for the error associated with the bilogarithmic methods.

Figure 5-9 shows a case where the curve did not follow exactly the same path as before unloading. The error for this test was $-2.95 \%$, a relatively higher compared to the ones from the bilogarithmic methods. A possible explanation for this behaviour is that unloading occurred before the straight line of Zone III had fully developed.

Unfortunately, when samples are retrieved from below the ground, the unloading stress path is not known, so it is necessary to rely on the reloading part of the curve for these tests. This is less accurate, and requires the user to select the points used to obtain the straight line based on judgement.

It should be mentioned that, although the bilogarithmic methods give the most accurate result for the loading test, they do not take sample disturbance into account. Thus, some method of quantifying sample disturbance should be used to apply a correction to the result obtained using these methods. 


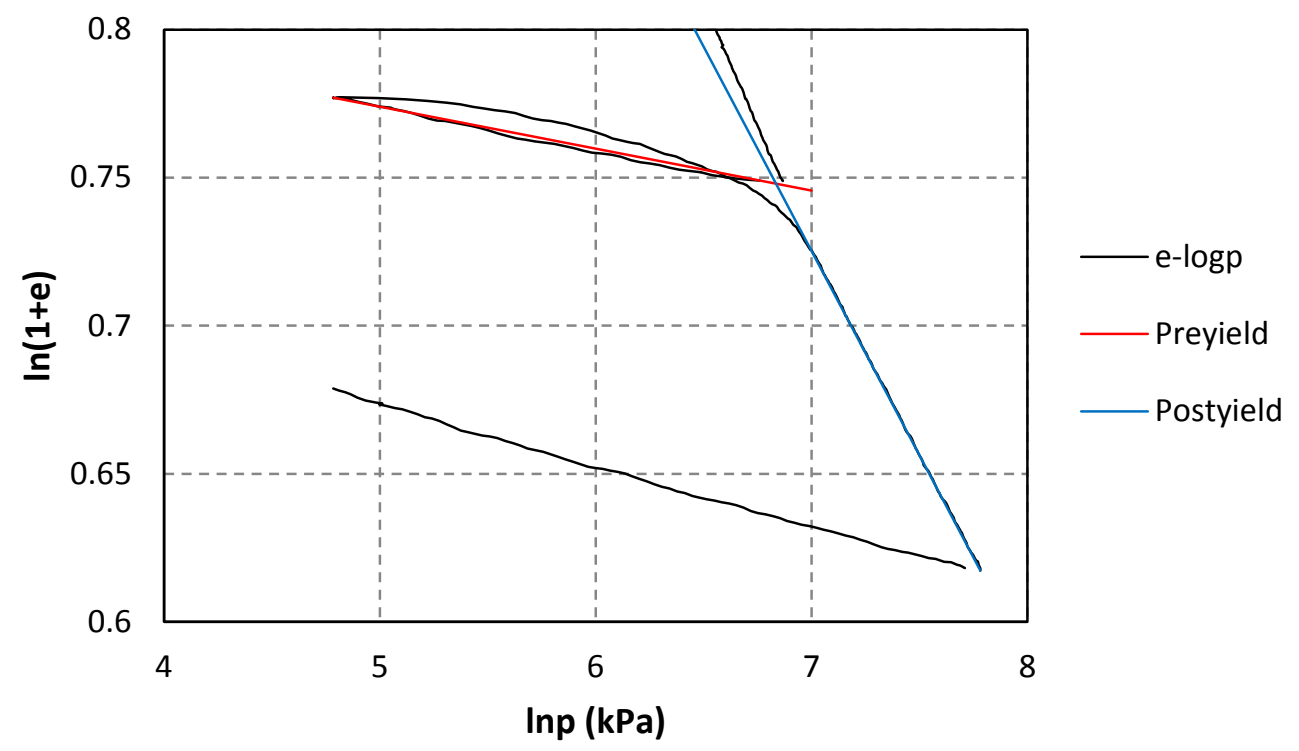

Figure 5-13: Example of a test where the consolidation curve during reloading follows a different path than before unloading

\subsection{8 e-logp Bilinear Method}

The procedure of the $e$-logp bilinear method follows the same principle as the bilogarithmic methods. That is, the pre-yield and post-yield parts of the consolidation curve are extended such that they intersect to find the preconsolidation pressure. The only difference is that the $e$-logp bilinear method involves plotting the consolidation data on an $e$-logp curve rather than on a $\log (1+e)$-logp curve.

For the reloading tests, it was found that the $e$-logp bilinear method did not perform quite as well as the bilogarithmic methods. This is seen in the higher error associated with this method ($2.87 \%$ as opposed to $-0.49 \%$ ). It is somewhat surprising that the two methods give such different errors, considering the close similarity between the two methods. Moreover, the pre-yield and post-yield lines were determined based on the same points for both the $e$-logp bilinear and bilogarithmic methods, which makes it even more surprising that the methods give such different results. This might be evidence that the bilogarithmic plot does in fact result in straighter lines for the pre-yield and post-yield regions than the $e$-logp plot.

It can be demonstrated graphically that the bilogarithmic plot is not equivalent to the $e$-logp plot. This is shown in Figure 5-14, where $e$-logp curve is shown in black and the $\log (1+e)$-logp curve is shown in red. The vertical scales are adjusted so that the maximum and minimum points for both plots coincide. 


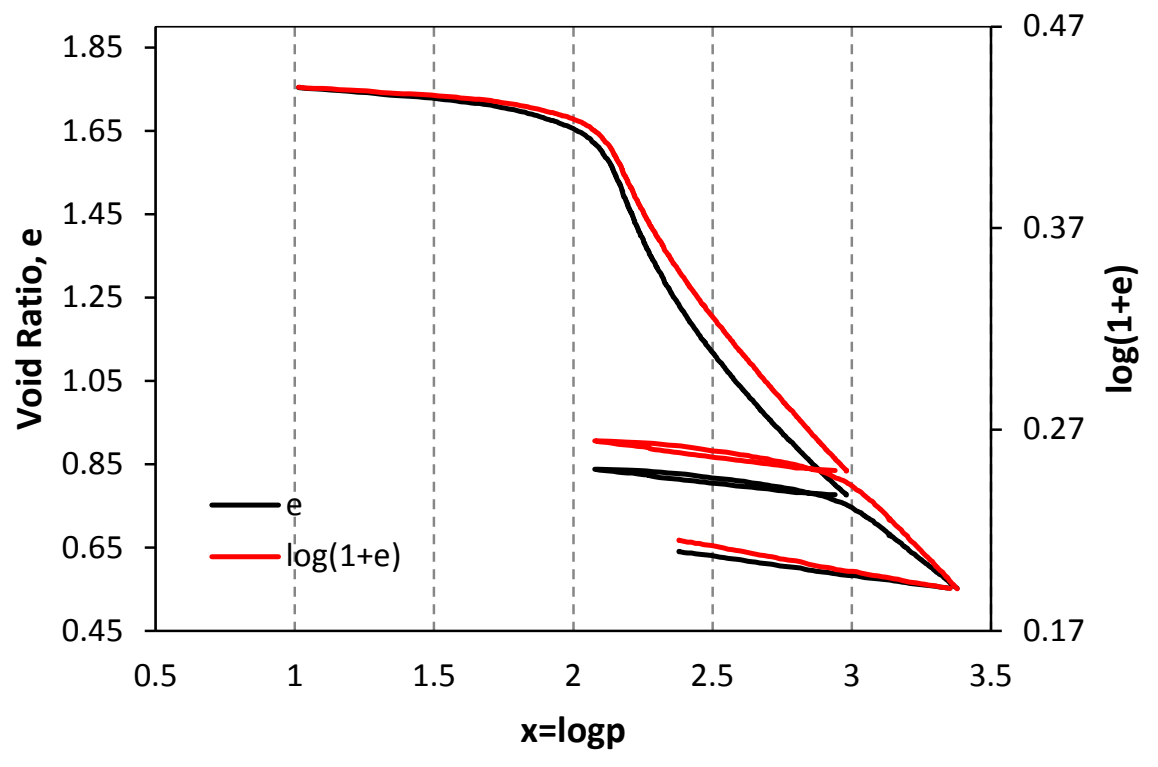

Figure 5-14: Comparison of e-logp and $\log (1+e)-\log$ plots for the same test

The bilogarithmic methods typically yield a higher value than the $e$-logp bilinear method. This is possibly explained by the apparently higher slope of the post-yield line observed in the figure.

During the initial loading stages, both the $e$-logp bilinear method and the work method gave the smallest percent difference $(-0.942 \%$ and $+1.065 \%$, respectively) of all the methods investigated when compared to the $\ln (1+e)$-lnp bilogarithmic method. This demonstrates that the $e$-logp bilinear and work methods are more similar to the bilogarithmic methods than any of the other methods. This is a reflection of the extremely similar nature of these three methods, in that they define preconsolidation pressure as the intersection of pre-yield and post-yield lines.

Once again, the close resemblance in the results of these two methods is in part aided by the fact that the same points were selected for determining the slope for both methods. Furthermore, the fact that the results for these methods are not identical provides evidence that these methods are not mathematically equivalent.

\subsubsection{Sallfors Method}

The results of the Sallfors method during reloading stages were rather inaccurate, with an average error on the same as the Janbu, Peck and Nagaraj et al. methods. The Sallfors method tended to underpredict the preconsolidation pressure.

During the initial loading stages, the Sallfors method was once again in the bottom three of the methods that behave similarly to the work and $\ln (1+e)$ - $\ln p$ methods. This reflects the fact that the Sallfors method is based on a very different philosophy than those two methods. 


\subsubsection{Janbu Method}

Before discussing the results of the tests, a comment will first be made regarding an observation of the Janbu method. Although this method is similar in concept to the other methods in the fact that it measures a change in the stiffness response of the soil after a certain stress is surpassed, the manner in which it measures this change is different.

It can be noted that since $D=\frac{1}{m_{v}}$ and $m_{v}=\frac{\Delta \epsilon}{\Delta \sigma^{\prime}}$, the plot of $D-p$ employed in the Janbu method will look similar to an e'(x)-p plot that is used to locate the inflection point. Thus, the stress obtained using the method of Janbu is expected to be very close to the point of inflection. Figure 5-15 illustrates this point, where the constrained modulus is shown in black and the slope of the e-logp curve is shown in red. The solid lines indicate that the range over which the minimum value for both curves can be found is similar for the two curves.

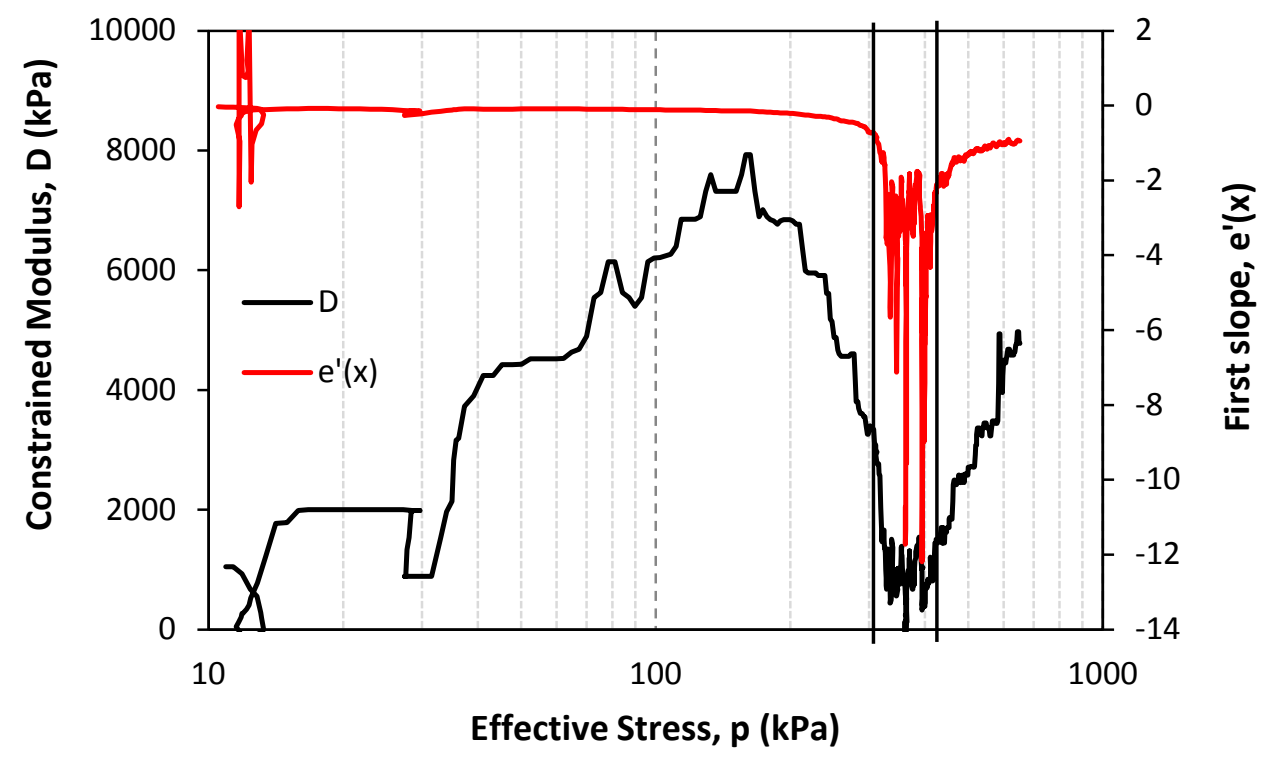

Figure 5-15: Comparison of constrained modulus with slope of $e$-logp curve

This gives validity to the method proposed by Karlsrud, who defined preconsolidation pressure as halfway between where strain softening begins and where strain hardening begins. This would shift the preconsolidation pressure obtained from the $D-p$ plot to the left of the inflection point. Thus, the method of Karlsrud (1991) probably gives a result closer to the true preconsolidation pressure than the method proposed by Janbu (1969). However, this would be a statistical correlation rather than theoretically based in mechanical phenomena. The Karlsrud method was not included in this investigation. 
In Figure 5-16, the pre-yield and post-yield lines of the Janbu D-logp plot are highlighted. It is shown that these straight line sections do not coincide with the pre-yield and post-yield lines of the e-logp plot.

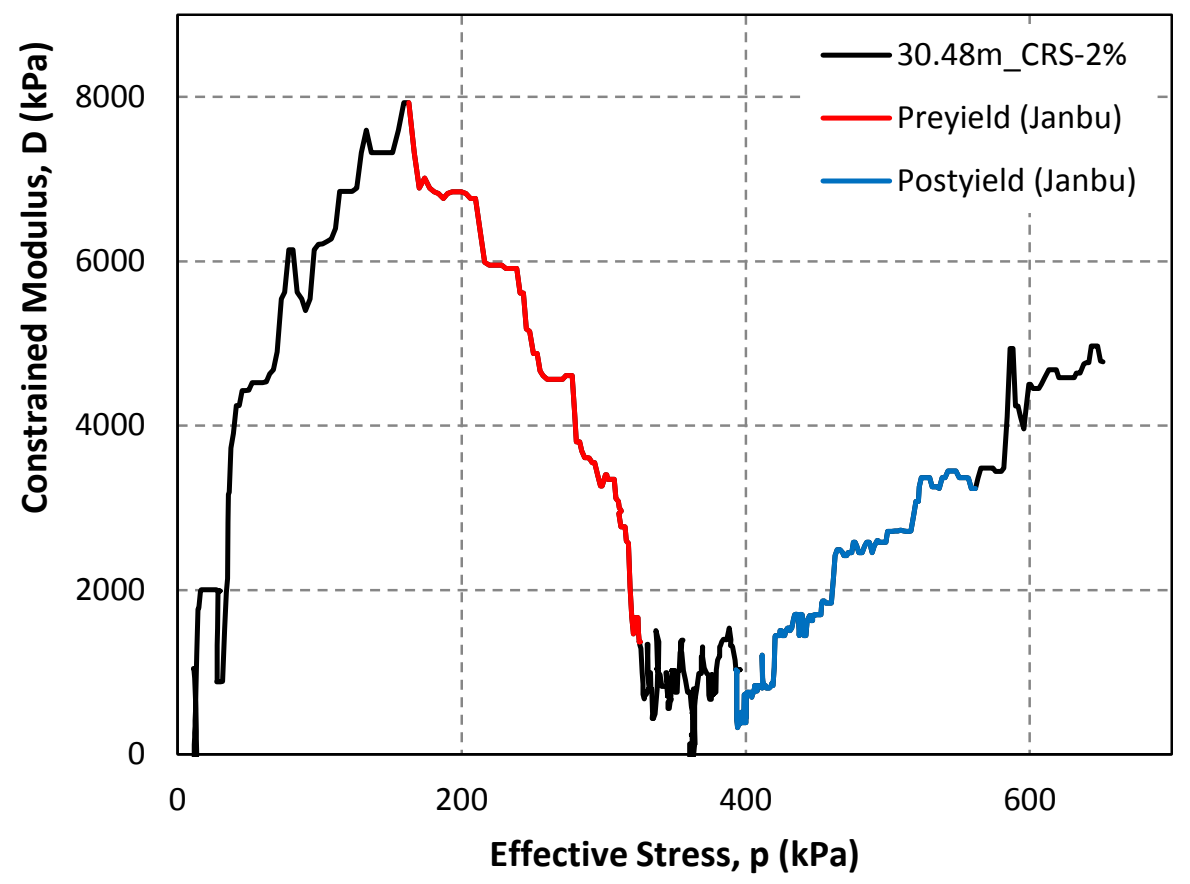

Figure 5-16: Locations of pre-yield and post-yield regions on $D-p$ plot

The figure below connects on the $e$-logp plot the points corresponding to the lower and upper bounds of the regions highlighted in the figure above. This demonstrates that the pre-yield and post-yield sections of the $e$-logp curve are different from those in the $D-p$ curve. 


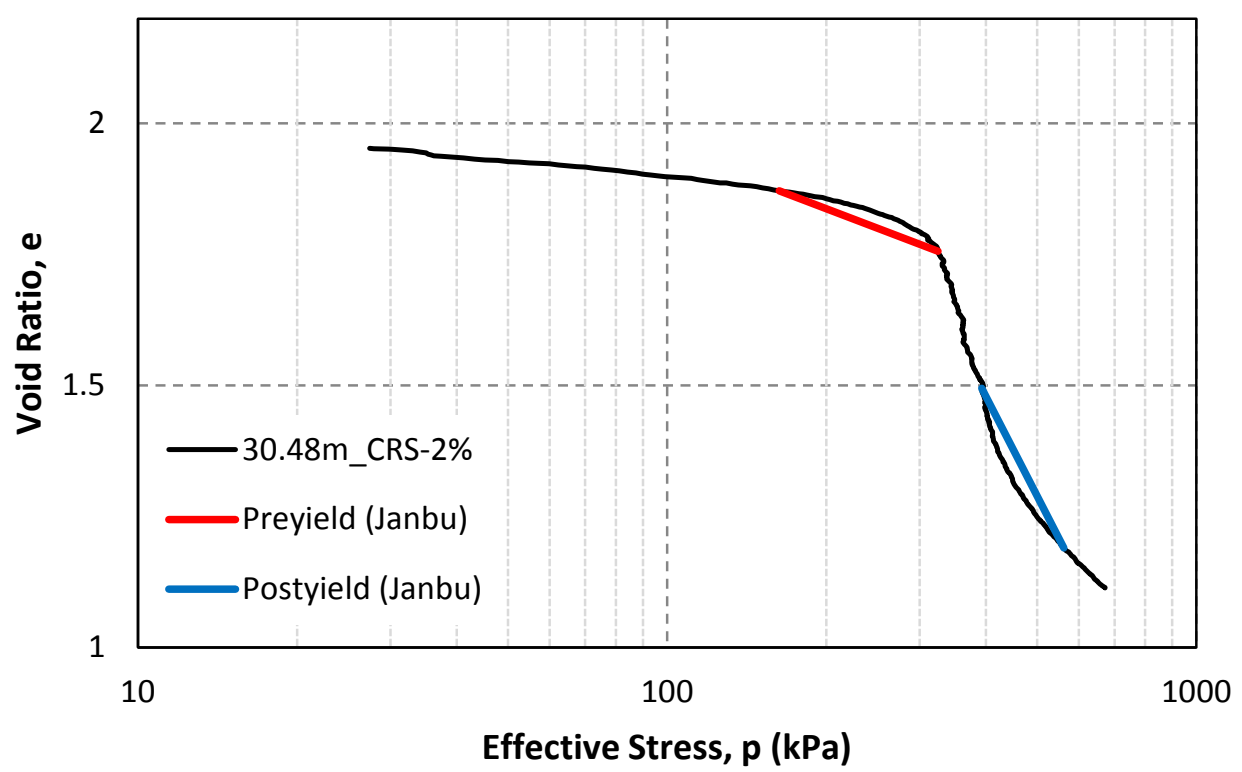

Figure 5-17: Regions of $D-p$ straight lines shown on corresponding e-logp plot

This illustrates the fact that the straight line portions of the $D-p$ plot are not equivalent to the straight line parts of the $e$-logp plot. Thus, the Janbu method will give different (higher) results than any of the other methods that involve locating the intersection of pre-yield and post-yield lines (i.e. the Work, bilogarithmic and e-logp bilinear methods).

The preceding discussion explains why the Janbu method resulted in a very large average error (16.60\%) during the reloading tests. The method tended to greatly overestimate the preconsolidation pressure.

Another drawback of the Janbu method is that considerable judgement is required in selecting the range of data over which to perform the regression to obtain each straight line. Because of the scatter that is still present even in the smoothed data, slight adjustments in the selected range can result in significant differences in the slope of the line and thus in the obtained preconsolidation pressure. This is consistent with the conclusion of Grozic et al. (2003) that the Janbu method is the most difficult to apply. However, they came to this conclusion for a different reason, which was the influence of sample disturbance on oedometer test results.

\subsection{Evaluation Of Numerical Methods}

A number of methods were devised to aid in the analysis of consolidation test data using graphical procedures. These include numerical methods to obtain the inflection point and the point of maximum curvature of the e-logp curve. Additionally, rather than graphically drawing the lines of best fit for straight line approximations, these lines were drawn by selecting two points along the straight line parts and plotting a straight line directly according to these points. 
The equations of the lines were then derived and the intersection of two lines was calculated to obtain the associated preconsolidation pressure.

In the case of the Janbu method, linear regression was used along the straight line parts of the plot to obtain the equations of the lines. The intersection of these straight lines was then calculated similarly to the previous methods. Median smoothing was also used to account for the scatter in the $D-p$ plots required for the Janbu method.

The purposes of these numerical methods were 1) to reduce the subjectivity of the graphical methods and make them more reproducible, and 2) to improve the precision with which the methods can be used.

The numerical method of determining the point of maximum slope and point of maximum curvature is useful in cases where such a point can be easily determined. However, this method should not be used without visually checking the validity of the point of maximum curvature and both the point and value of maximum slope.

Indeed, the locations of the inflection point and the point of maximum curvature could be located to within a narrow range. The key barrier to using the numerical methods exclusively to find the point and value of maximum slope and maximum curvature is the existence of local fluctuations in the $e$-logp curve. These fluctuations are due in part to fluctuation of the load frame sensor and in part to sample preparation errors. It is corrected to an extent by using a range (in this investigation 21 points) to calculate the first and second slopes rather than using adjacent points. However, a degree of fluctuation is still seen in the curvature plot, making the method unreliable in some cases.

In a number of cases, the slope found at the inflection point was considered unreliable by visual inspection, and the maximum slope had to be interpreted graphically. In such cases, two points were selected along the straight line portion of the curve over a range greater than 21 points that appeared to give the closest approximation of the highest slope.

One observation is that the plot of maximum curvature vs. logarithm of effective stress ( $\kappa$-logp) exhibits a curvilinear trend up to a certain point, and then becomes much more scattered. It was found that, in cases where the location and magnitude of maximum curvature was not reasonable, the plot could still be used by taking the point just before the data deviates from this curvilinear trend. The large amount of scatter after this point is attributed to the fact that the slope of the e-logp curve after the point of maximum curvature becomes nearly vertical. Thus, both the first and second slope, and therefore the curvature, obtained from the $e$-logp curve become more affected by small fluctuations in the $\mathrm{x}$-value. The figure below shows an example of a case where the point of maximum curvature had to be selected based on judgement rather than solely relying on the maximum absolute value of curvature. 


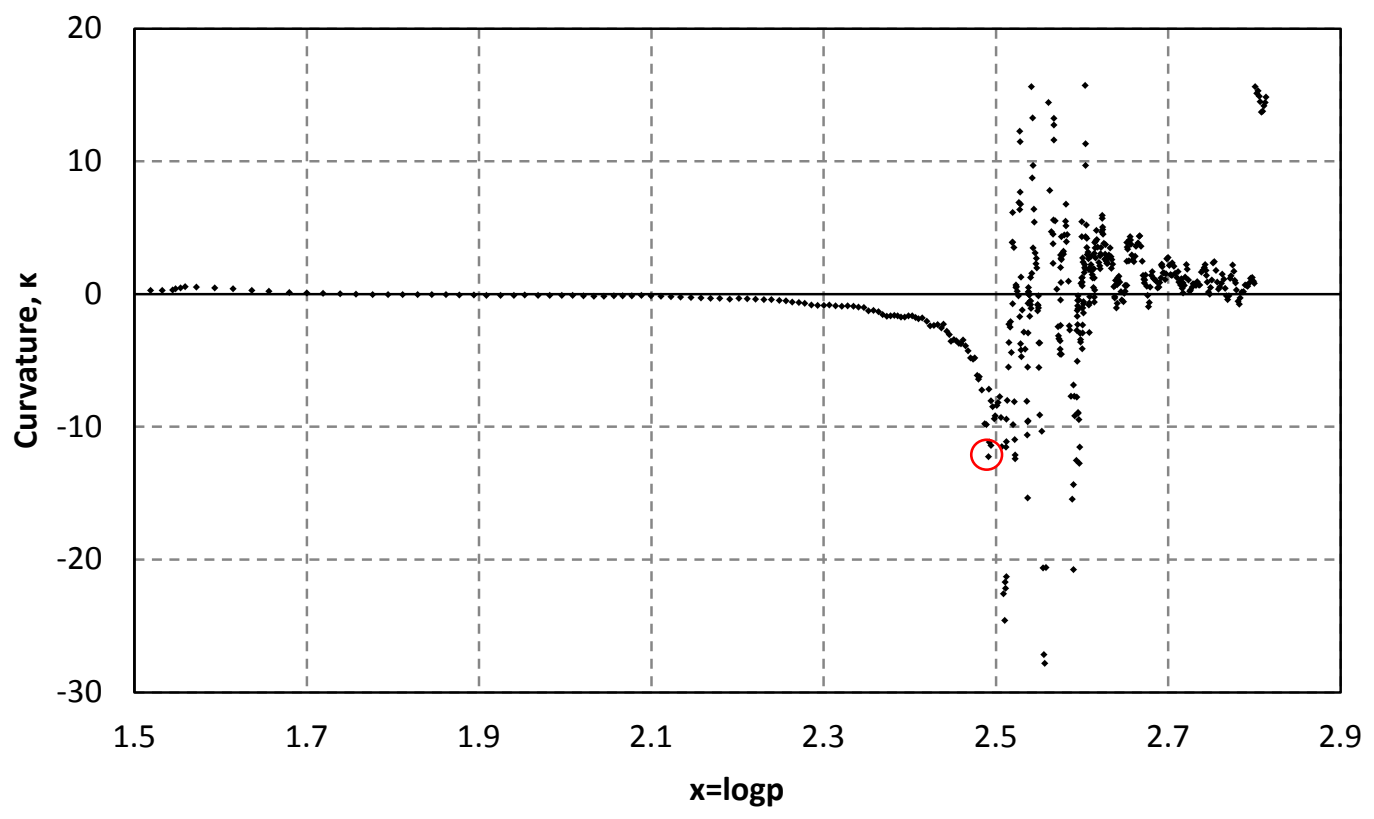

Figure 5-18: Selection of point of maximum curvature in a case where judgement is required

Table 5-5 summarizes how the point of maximum curvature and the inflection point were selected for each test. The notation "Numerical" indicates that the numerical method was used exclusively to locate the point of interest. The notation "Visual" indicates that the numerical method was not able to give a reliable result, so the points of maximum curvature and the slope of Zone II had to be obtained visually.

Table 5-5: Summary of methods used to determine maximum curvature and inflection points for all tests

Initial Loading

\begin{tabular}{|c|c|c|}
\cline { 2 - 3 } \multicolumn{1}{c|}{} & $\begin{array}{c}\text { Maximum } \\
\text { Curvature }\end{array}$ & $\begin{array}{c}\text { Maximum } \\
\text { Slope }\end{array}$ \\
\hline $11.36 \mathrm{~m}-0.5 \%$ & Numerical & Numerical \\
\hline $11.36 \mathrm{~m}-1 \%$ & Numerical & Numerical \\
\hline $15.60 \mathrm{~m}-0.5 \%$ & Numerical & Numerical \\
\hline $15.60 \mathrm{~m}-1 \%$ & Visual & Visual \\
\hline $\begin{array}{c}15.60 \mathrm{~m}- \\
1 \% \_k x\end{array}$ & Numerical & Numerical \\
\hline $15.60 \mathrm{~m}-5 \%$ & Numerical & Numerical \\
\hline $30.48 \mathrm{~m}-1 \%$ & Numerical & Numerical \\
\hline $30.48 \mathrm{~m}-2 \%$ & Numerical & Visual \\
\hline $34.90 \mathrm{~m}-$ & Numerical & Numerical \\
\hline
\end{tabular}

Reloading

\begin{tabular}{|c|c|c|}
\cline { 2 - 3 } \multicolumn{1}{c|}{} & $\begin{array}{c}\text { Maximum } \\
\text { Curvature }\end{array}$ & $\begin{array}{c}\text { Maximum } \\
\text { Slope }\end{array}$ \\
\hline $11.36 \mathrm{~m}-0.5 \%$ & Numerical & Numerical \\
\hline $11.36 \mathrm{~m}-1 \%$ & Numerical & $\mathrm{n} / \mathrm{a}$ \\
\hline $15.60 \mathrm{~m}-0.5 \%$ & Numerical & $\mathrm{n} / \mathrm{a}$ \\
\hline $15.60 \mathrm{~m}-1 \%$ & Visual & $\mathrm{n} / \mathrm{a}$ \\
\hline $15.60 \mathrm{~m}-$ & Numerical & $\mathrm{n} / \mathrm{a}$ \\
\hline $1 \% \_\mathrm{kx}$ & & $\mathrm{n} / \mathrm{a}$ \\
\hline $15.60 \mathrm{~m}-5 \%$ & Numerical & $\mathrm{n} / \mathrm{a}$ \\
\hline $30.48 \mathrm{~m}-1 \%$ & Numerical & $\mathrm{n} / \mathrm{a}$ \\
\hline $30.48 \mathrm{~m}-2 \%$ & Numerical & $\mathrm{n} / \mathrm{a}$ \\
\hline $39.48 \mathrm{~m}-1 \%$ & Numerical & \multicolumn{2}{c|}{} \\
\hline
\end{tabular}




\begin{tabular}{|c|c|c|}
\hline $1 \% \_k x$ & Numerical & Numerical \\
\hline $\begin{array}{c}39.48 m- \\
1 \% \_k x\end{array}$ & Numerical & Visual \\
\hline $39.48 m-1 \%$ & $1 / 11$ & $3 / 11$ \\
\hline Visual Total & $10 / 11$ & $8 / 11$ \\
\hline $\begin{array}{c}\text { Numerical } \\
\text { Total }\end{array}$ & \\
\hline
\end{tabular}

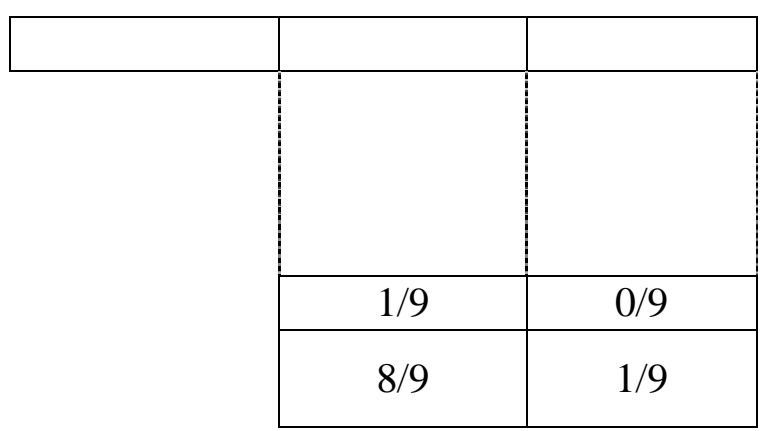

In all tests but one (11.36m-0.5\%), the unloading/reloading cycle occurred in Zone III of the $e$ logp curve. Thus, upon reloading, there is no inflection point for the $e$-logp curve to demonstrate. After surpassing the maximum past load, the curve simply resumes the straight line of Zone III. The slope of this line is used, which is why it was not necessary to determine the inflection point numerically for the reloading tests.

Although the location of the points of interest can be found with some degree of precision using these methods, it is not clear how accurate or representative the values of these parameters are at their points of maximum value due to the influence of local fluctuations. A visual check is always required to ensure that the slope of the e-logp curve at the inflection point according to this method makes sense.

In the cases where the numerical method could be used to locate the inflection point of the $e$-logp curve, a similar procedure was used to find the inflection point of the $W-p, \log (1+e)-\log p$ and $\ln (1+e)$ - $\ln p$ curves. For example, for the $\ln (1+e)-\ln p$ plot, the following formula was used.

$$
\ln (1+e)^{\prime}(\ln p)=\frac{\ln (1+e)_{i+\Delta i}-\ln (1+e)_{i-\Delta i}}{\ln p_{i+\Delta i}-\ln p_{i-\Delta i}}
$$

After performing this analysis, it was found in nearly every case that the same point is identified as the inflection point for a given test using the $e$-logp curve, the bilogarithmic curves and the $W$ p curve. For example, if 2050 data points are collected during a CRS test, the numerical method might identify the $307^{\text {th }}$ point (and the corresponding effective stress) as the inflection point for all the methods. This is a valuable insight that is only possible due to the use of this numerical method.

The methods of determining preconsolidation pressure investigated in this study are inevitably graphical in nature. However, by using these numerical methods as tools, the accuracy and objectivity of these methods can be greatly improved. Moreover, the results obtained using the graphical methods are more repeatable. 


\subsection{INFLUENCE OF LOADING STRAIN RATE}

Past research on the influence of loading strain rate on consolidation tests have shown that, as the strain rate increases, the preconsolidation pressure obtained from a CRS test is expected to also increase. At a number of depths, tests were performed at multiple strain rates, so it can be investigated whether or not this pattern emerges.

Although little test data is available from which to draw correlations regarding strain rate influence, it can be seen higher preconsolidation pressures are indeed obtained when higher strain rates are employed at the same depth.

Table 5-6 lists the preconsolidation pressure obtained by the bilogarithmic methods for those depths where multiple strain rates were employed.

Table 5-6: Influence of loading strain rate on preconsolidation pressure

\begin{tabular}{|l|l|l|l|l|}
\hline & \multicolumn{4}{|c|}{ p'c by bilogarithmic Method } \\
\hline Depth & $0.5 \% / \mathrm{hr}$ & $1 \% / \mathrm{hr}$ & $2 \% / \mathrm{hr}$ & $5 \% / \mathrm{hr}$ \\
\hline $11.36 \mathrm{~m}$ & $114.21 \mathrm{kPa}$ & $121.51 \mathrm{kPa}$ & & \\
\hline $15.6 \mathrm{~m}$ & $128.59 \mathrm{kPa}$ & $131.86 \mathrm{kPa}$ & & $140.53 \mathrm{kPa}$ \\
\hline $30.48 \mathrm{~m}$ & & $267.23 \mathrm{kPa}$ & $304.56 \mathrm{kPa}$ & \\
\hline
\end{tabular}

This data is represented graphically in Figure 5-19. A logarithmic horizontal scale is chosen for convenience, due to the large gap in loading strain rates. 


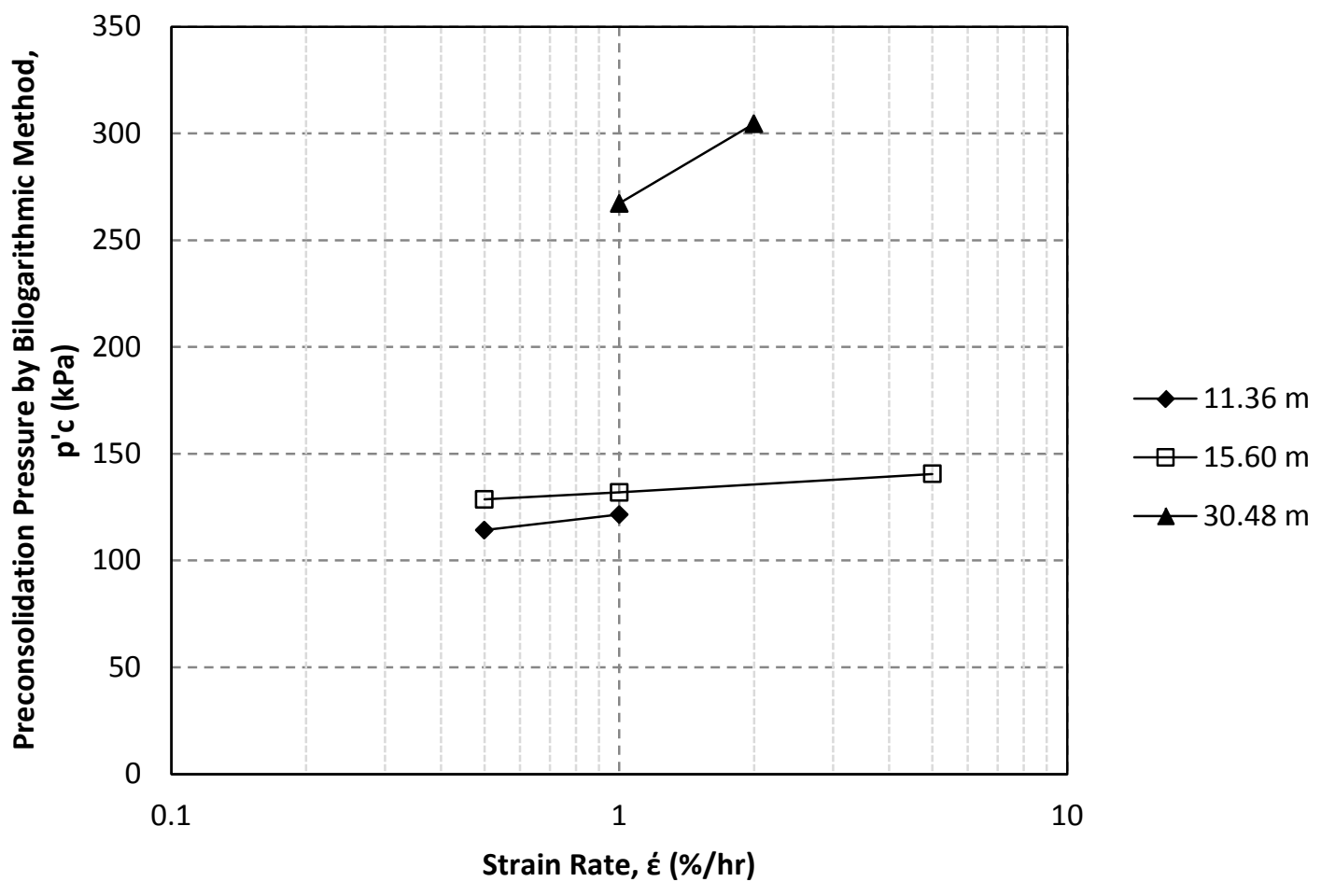

Figure 5-19: Influence of strain rate on preconsolidation pressure from CRS tests

\subsection{INFLUENCE OF SAMPLE ORIENTATION}

In order to satisfy the needs of the project with which the current research is associated, samples were prepared in two different orientations: horizontal and vertical. Those prepared in a vertical orientation were trimmed in such a way that the direction in which force is applied during the CRS test is transverse to the vertical direction in the field. Those prepared in a horizontal orientation were prepared such that the orientation during loading is the same as it is in the field.

From this investigation, one can compare the influence of sample orientation on preconsolidation pressure. Although tests are not available in both orientations for all depths, the relationship between horizontal and vertical loading can still be investigated by plotting the preconsolidation pressure vs. depth for each test. Two straight lines can then be produced, one connecting the tests in the horizontal orientation, and one connecting those in the vertical orientation. The slope of each line represents the increase of preconsolidation pressure with depth. The ratio between the slopes of the lines for the vertical and horizontal orientations can be represented as follows:

$$
\frac{\sigma_{v}^{\prime}}{\sigma_{h}^{\prime}}=\frac{0.1096}{0.1501} \approx 0.73
$$




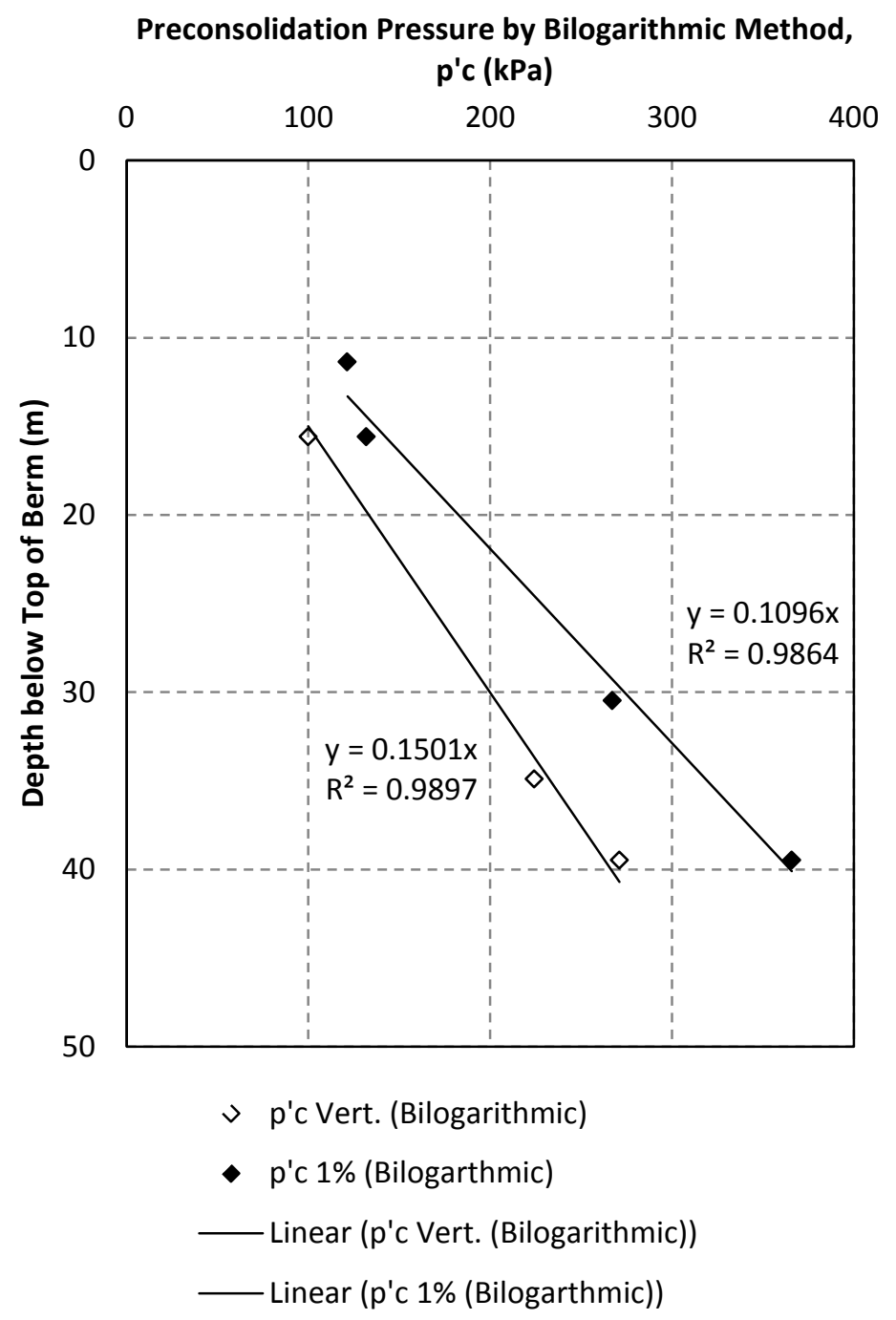

Figure 5-20: Influence of sample orientation on preconsolidation pressure

\subsection{SUMMARY}

The results of each of the eleven graphical methods were presented and discussed in this section.

When applying the graphical methods to reloading stages, the methods were evaluated by comparing the preconsolidation pressure obtained by those methods to the known maximum past pressure. This was done by analyzing the regression coefficient $\left(\mathrm{R}^{2}\right)$ and the average percent error for each method. It was found that the bilogarithmic methods most accurately reproduced the preconsolidation pressure in the reloading stages. 
In the initial loading stages, it wasn't possible to compare the preconsolidation pressure obtained by the different methods to any known maximum past pressure. Thus, the results of the different methods were compared first to the work method and then to the $\ln (1+e)-\ln p$ bilogarithmic method.

An evaluation of the numerical methods that were developed to aid in the interpretation of the test data by the graphical methods was also presented. It was found that the method to locate the point of maximum curvature is very effective in cases where it can easily be applied. However, due to scatter in the test data, some user interpretation is still sometimes required. The method of drawing straight lines for the graphical methods through plotting equations was found to be extremely effective in giving the user control over how to draw the line, as well as in reducing error from interpreting preconsolidation stress visually from a graph. Furthermore, this approach to drawing the lines allowed valuable insights such as the identical results yielded by the two bilogarithmic methods.

Similar trends were observed for both the initial loading and reloading cases. This suggests that any methods that are suitable for the reloading cases are also suitable for the initial loading cases. Thus, because of the high accuracy of the bilogarithmic methods in the reloading cases, it is suggested that this method is also most suitable for application to undisturbed samples. However, because this method does not account for sample disturbance, some correction factor should be used when employing this method. 


\section{CONCLUSIONS AND FUTURE RESEARCH}

\subsection{INTRODUCTION}

Identifying the preconsolidation pressure of a soil is an important challenge in geotechnical engineering. Many methods have been developed to identify this pressure from consolidation test data, and the vast majority of these methods are graphical in nature. Sensitive clay is a soil type that presents unique challenges in accurately identifying the preconsolidation pressure. This is due to the "S-shape" of the $e$-logp curve, as well as to the tendency of the soil to become disturbed during sampling, handling and testing, thus distorting the consolidation test data.

In this study, a total of eleven different methods (including one that is not found in the literature) for determining preconsolidation pressure are applied to 11 CRS tests. Nine of these 11 tests included reloading stages on which the methods could also be applied for performance evaluation. During these reloading stages, the maximum past pressure was known and the result of the different methods could be evaluated based on their accuracy in reproducing that pressure. In the initial loading stages, the maximum past pressure was not known, so the different methods were compared to each other.

The graphical methods to determine preconsolidation pressure included in this study are:

- Casagrande method

- Peck method

- Pacheco Silva method

- Nagaraj et al. method

- Work method

- Wang and Frost method

- Bilogarithmic methods

o $\log (1+e)-\log p$

$0 \quad \ln (1+e)-\ln p$

- e-logp Bilinear method

- Sallfors method

- Janbu method

All of the above methods are graphical in nature. Thus, these methods have difficulties such as locating the point of maximum curvature and inflection point or consistently drawing the same slope when repeating a test on the same data set. Furthermore, the graphical nature of these methods introduces sources of error such as interpreting preconsolidation pressure visually on the effective stress axis. Numerical methods are developed in this study to aid the interpretation of test data using these graphical procedures. These include methods to locate the point of 
maximum curvature and inflection point of an $e$-logp curve (developed for this study), as well as plotting straight lines using equations rather than drawing them visually, a concept that was introduced by Boone (2010).

\subsection{ConClusions}

Among the eleven methods included in the study, the bilogarithmic methods gave the closest comparison with the known maximum past pressure during the reloading stage study. This is consistent with the findings of past researchers. Furthermore, it was found that two variations of the bilogarithmic method $(\log (1+e)-\log p$ and $\ln (1+e)-\ln p)$ gave identical results, provided that the same data points were used to draw the pre-yield and post-yield lines. This validates the claim by past researchers that the different variations of the bilogarithmic method are mathematically equivalent.

Very similar preconsolidation pressures were obtained by three methods that define it as the intersection of pre-yield and post-yield lines: the work method, the $e$-logp bilinear method, and the bilogarithmic method. The results of these three methods were not identical, suggesting that they are not mathematically equivalent. Of these three methods, the work method performed the least accurately.

The Wang and Frost method gave a lower average error than the work method. This validates the claim of those authors that using dissipated strain energy is more appropriate than total strain energy, as is the case with the work method. However, Umar and Sadrekarimi (2016) found the opposite result.

The Casagrande method was found to be fairly inaccurate in reloading tests compared to the other methods, and it overestimated the preconsolidation pressure. This is consistent with the results of past researchers. The Pacheco Silva method was found to give a fairly good approximation of the preconsolidation pressure, performing better than both the Casagrande and work methods.

The methods that gave the highest average error were those of Nagaraj et al. (overestimated), Peck (underestimated) and Janbu (overestimated). For the first two methods, the reason is that the cementation bonds of sensitive clay upon which they are based had been broken during the reloading stage. In fact, these two methods are not truly applicable during reloading, but they were nevertheless applied to the reloading tests for purposes of comparison with the other methods. It was shown numerically that the result of the Janbu method should be close to the inflection point, which explains the high degree of overestimation for this method.

For the initial loading stages, it was found that the Nagaraj et al. method did not correspond well with the other methods. The purpose of this method is to determine the field preconsolidation pressure, so this could be a reflection of varying degrees of sample disturbance between the tests. 
Although the bilogarithmic method gave the most accurate results when compared with known maximum past pressures, this does not guarantee that it is the most suitable method for laboratory samples. The high accuracy obtained with this method is based on reloading tests where the sample had not experienced any disturbance other than stress release. Laboratory samples typically have an unknown degree of disturbance, which should be taken into account when using the bilogarithmic method.

A new procedure of numerically determining the point of maximum curvature and point of inflection was investigated. The method was found to be objective and precise, but only in cases where it could easily be applied. Some user interpretation was still required to determine if the points that are calculated as having the maximum slope or maximum curvature can truly be used for those purposes. The cause of this subjectivity was the scatter that is present in the $e$-logp data.

In this investigation, the graphical procedures were performed by plotting the necessary straight lines using equations based the slope of the curve at a given point and the coordinates of that point on the curve. Plotting the lines in this way was found to be very convenient to execute. Furthermore, it allows a great deal of control for the user in choosing how to draw the necessary lines, thus improving the precision and repeatability with which the graphical methods are performed. This approach does not change the fact that the methods are graphical in nature.

The use of the numerical procedures illuminated certain features of the different graphical methods. First, it was demonstrated numerically that the point of maximum curvature is moved to the left when the ratio of vertical scale to horizontal scale is exaggerated. Second, they revealed that the bilogarithmic methods give identical results when based on the same data points. Finally, it is only due to the precision granted by these procedures that it can be said with confidence that the $e$-logp bilinear method and Work method do not give results identical to those of the bilogarithmic methods.

\subsection{FUTURE WORK}

All of the graphical procedures evaluated in this study are based on the observation that soil experiences a change in stiffness around the preconsolidation pressure. However, methods based on other principles, such as correlations with shear strength, shear wave velocity, and change in pore pressure response also exist. These different classifications of methods should be studied in tandem to determine their relationship with one another. More significantly, the methods studied are all graphical in nature. More work should be done to predict preconsolidation pressure based on physical processes rather than graphical estimations.

In this study, the accuracy of the different methods to determine preconsolidation pressure was evaluated based on reloading curves, where the only source of disturbance was from stress release. Past work has been done to attempt to quantify sample disturbance, and the Nagaraj et al. method is an example of a graphical method that takes sample disturbance into account. 
However, the influences of sample disturbance on the preconsolidation pressure should be further investigated in the future.

Throughout this study, a value of 1 was assumed for the ratio of $e$ to logp when determining the point of maximum curvature based on the newly developed numerical method. This is equivalent to an increment of 1 on the void ratio axis being drawn the same length as one log cycle on the stress axis. However, it was demonstrated that when a scale factor is to exaggerate the vertical scale, the point of maximum curvature moves to the left and the value of curvature reduces. A parametric study should be performed to determine whether or not a ratio of 1 is appropriate.

Throughout the study, the equations of the straight line portions of $e$-logp curves were found by selecting two points such that the line connecting those points appears to closely fit the data. However, the pre-yield and post-yield lines can also be obtained by linear regression. Similarly, regression can be used to investigate the claim that the bilogarithmic plot results in straighter pre-yield and post-yield regions than the $e$-logp curve. Given the large number of data points available from a CRS test, this can be easily done in future studies.

The scatter in the $e$-logp data is probably the result of fluctuation in the CRS machine sensors. This scatter greatly influences the slope and maximum curvature plots, and thus leads to a degree of subjectivity still being present in interpreting the points of maximum curvature and inflection. The numerical methods should be applied to test data where sensor fluctuation was not significant during the test in order to further evaluate the validity of these methods.

A short computer script can easily be created based on the numerical methods described in this study to perform the graphical methods by simply inputting CRS data. In fact, it is technically possible to perform the graphical methods without creating any plots, although the results of such a script should never be used without graphically confirming the validity its results.

No attempt has been found in the literature to quantify the maximum curvature, and thus no relationships are known to exist between curvature and other parameters. The proposed method to determine the value of curvature at the maximum point can be used to study the relationship between magnitude of maximum curvature and parameters such as sample disturbance and preconsolidation pressure.

Finally, all of the methods investigated make simplifying assumptions about the behaviour of soil, mostly that the pre-yield and post-yield regions form straight lines. However, consolidation tests consistently show that the $e$-logp plots of consolidation data show some degree of curvature in these regions of the consolidation curve. A significant discussion has not been found in the literature on developing a function to describe the e-logp curve of a soil sample. If such a function is developed, a new constitutive model that does not rely on the assumption of linear compression indices can be developed based on this function. The numerical methods to define the stress at the inflection point and at maximum curvature, as well as the values of maximum 
slope and maximum curvature, can be applied to CRS test data, and these values can be used to when calibrating the function based on test data. 


\section{BIBLIOGRAPHY}

Acres. (1976). Waba Dam Geotechnical Design - Arnprior GS. Acres Consulting Ltd.

Adams, A. (2011). Laboratory Evaluation of the Constant Rate of Strain and Constant Head Techniques for Measurement of the Hydraulic Conductivity of Fine Grained Soils. Massachussets Institute of Technology.

Alshawmar, F. (2014). Evaluation of Compressibility, Anisotropy and At-rest Lateral Earth Pressure in Champlain Sea Clays (thesis). Ottawa: Carleton University.

ASTM. (2012). One-Dimensional Consolidation Properties of Saturated Cohesive Soils Using Controlled-Strain Loading.

Becker, D., Crooks, J., Been, K., \& Jeffries, M. (1987). Work as a Criterion for Determining In Situ and Yield Stresses in Clays. Canadian Geotechnical Journal, 24, 549-564.

Benson, R., \& Morrison, N. (1975). Design of Waba Dam. Engineering Problems in Marine Clay.

Bjerrum, L. (1967). Engineering Geology of Norwegian Normally-Consolidated Marine Clays as Related to Settlements of Buildings. Geotechnique, 81-118.

Boone, S. J. (2010). A Critical Reappraisal of "Preconsolidation Pressure" using the Oedometer Test. Canadian Geotechnical Journal, 281-296.

Brand, E., \& Brenner, R. (1981). Soft Clay Engineering. Amsterdam: Elsevier Scientific Publishing Company.

Burland, J. (1990). On the Compressibility and Shear Strength of Natural Clays. Geotechnique, 40(3), 329-378.

Butterfield, R. (1979). A Natural Compression Law for Soils (an Advance on e-logp). Geotechnique, 469-480.

Casagrande, A. (1936). The Determination of Preconsolidation Load and its Practical Significance. Proceedings of the International Conference on Soil Mechanics and Foundation Engineering, 3, pp. 60-64.

Clayton, C., Matthews, M., \& Simons, N. (1995). Undisturbed Sampling Techniques. In Site Investigations (2 ed.). Oxford [England] Blackwell Science.

Clementino, R. (2005). Discussion of "An Oedometer Test Study on the Preconsolidation Pressure of Glaciomarine Clays". Canadian Geotechnical Journal, 972-974. 
Cragg, C. (1988). Waba Dam - Phase II Investigation - Laboratory Testing Report. Ontario Power Generation.

Crawford, C. (1968). Quick Clays of Eastern Canada. Engineering Geology, 2(4), 239-265.

Das, B. (2008). Advanced Soil Mechanics (3 ed.). New York: Taylor \& Francis.

DeGroot, D., Landon, M., \& Ryan, R. (2007). Objective Evaluation of Preconsolidation Stress for Soft Clays from Constant Rate of Strain Pore Pressure Data. GeoDenver. Denver: ASCE.

Feng, W. Q., Yin, J. H., \& Yin, Z. Y. (2014). Elasto-ViscoPlastic Model (EVP-Yin) as User Defined Soil Model for PLAXIS. Hong Kong Polytechnic University.

Grozic, J., Lunne, T., \& Pande, S. (2003). An Oedometer Test Study on the Preconsolidation Stress of Glaciomarine Clays. Canadian Geotechnical Journal, 857-872.

Hamilton, J., \& Crawford, C. (1959). Improved Determination of Preconsolidation Pressure of a Sensitive Clay. American Society for Testing Materials Special Technical Publication No. 254.

Hamilton, J., \& Crawford, C. (1960). Improved Determination of Preconsolidation Pressure of a Sensitive Clay. American Society for Testing Materials Special Technical Publication No. 254.

Hamouche, K., Leroueil, S., Roy, M., \& Lutenegger, A. (1995). In Situ Evaluation of K0 in Eastern Canada Clays. Canadian Geotechnical Journal, 677-688.

Holtz, R., \& Kovacs, W. (1981). An Introduction to Geotechnical Engineering. New Jersey: Prentice-Hall, Inc.

Hong, Z., \& Onitsuka, K. (1998). A Method for Correcting Yield Stress and Compression Index of Ariake Clays for Sample Disturbance. Soils and Foundations, 38(2), 211-222.

Janbu, N. (1969). The Resistance Concept Applied to Deformation of Soils. Proceedings of the 7th International Conference on Soil Mechanics and Foundation Engineering, 2529. Mexico City.

Janbu, N. (1981). Settlement Calculations Based on the Tangent Modulus Concept Part 1: Tangent Modulus.

Karlsrud, K., \& Hernandez-Martinez, F. (2013). Strength and Deformation Properties of Norwegian Clays from Laboratory Tests on High Quality Block Samples. Canadian Geotechnical Journal, 50, 1273-1293. 
La Rochelle, P., Sarrailh, J., Tavenas, F., \& Leroueil, S. (1981). Causes of Sampling Disturbance and Design of a New Sampler for Sensitive Soils. Canadian Geotechnical Journal, 5266.

Li, K. (1989). Work as a Criterion for Determining In Situ and Yield Stresses in Clays: Discussion. Canadian Geotechnical Journal, 26, 324-326.

Liu, J., Shi, C., \& Kirstein, A. (2016). Long-Term Settlement of Waba Dam: Progress Report. Toronto: Ryerson University.

Lunne, T., Berre, T., Andersen, K., Sjursen, M., \& Mortensen, N. (2006). Effects of Sample Disturbance on Consolidation Behaviour of Soft Marine Norwegian Clays. Canadian Geotechnical Journal, 43(7), 726-750.

Murthy, V. (2003). Geotechnical Engineering: Principles and Practices of Soil Mechanics and Foundation Engineering. New York: Marcel Dekker.

Nader, A. (2014). Engineering Characteristics of Sensitive Marine Clays - Examples of Clays in Eastern Canada. Ottawa: University of Ottawa.

Nagaraj, T., Murthy, B., Vatsala, A., \& Joshi, R. (1990). Analysis of Compressibility of Sensitive Soils. Journal of Geotechnical Engineering, 116(1), 105-118.

Oikawa, H. (1987). Compression Curve of Soft Soils. Soils and Foundations, 27(3), 99-104.

Onitsuka, K., Hong, Z., Hara, Y., \& Yoshitake, S. (1995). Interpretation of Oedometer Test Data for Natural Clays. Soils and Foundations, 35(3), 61-70.

Paniagua, P., L'Heureux, J.-S., Yang, S., \& Lunne, T. (2016). Study on the Practices for Preconsolidation Stress Evaluation from Oedometer Tests. Proceedings of the 17th Nordic Geotechnical Meeting. Reykjavik.

Peck, R., Hanson, W., \& Thornburn, T. (1974). Foundation Engineering (2 ed.). John Wiley \& Sons, Inc.

Prasad, K., Triveni, S., Schancz, T., \& Nagaraj, T. (2007). Sample Disturbance in Soft and Sensitive Clays: Analysis and Assessment. Marine Georesources \& Geotechnology, 25(3-4), 181-197.

Quigley, R., Gwyn, Q., White, O., Rowe, R., Haynes, J., \& Bohdanowicz, A. (1983). Leda Clay from Deep Boreholes at Hawkesbury, Ontario. Part I: Geology and Geotechnique. Canadian Geotechnical Journal, 20, 288-298.

Rankka, K., Andersson-Skold, Y., Hulten, C., Larsson, R., Leroux, V., \& Dahlin, T. (2004). Quick Clays in Sweden. Swedish Geotechnical Institute. 
Sallfors, G. (1975). Preconsolidation Pressure of Soft, High-Plastic Clays. Chalmers University of Technology Goteborg.

Salloum, T., Cragg, C., Bhardwaj, V., \& Hassan, P. (2009). Deformation Performance of Waba Dam. Canadian Geotechnical Society Conference, 62, pp. 20-24. Halifax.

Seah, T., \& Juirnarongrit, T. (2003). Constant Rate of Strain Consolidation with Radial Drainage. Geotechnical Testing Journal, 26(4).

Silvestri, V., Yong, R., Soulié, M., \& Gabriel, F. (1986). Controlled-Strain, Controlled-Gradient, and Standard Consolidation Testing of Sensitive Clays. (R. Yong, \& F. Townsend, Eds.) Consolidation of Soils: Testing and Evaluation, ASTM STP 892, 433-450.

Skempton, A., \& Northey, R. (1952). The Sensitivity of Clays. 3, 30-53.

Smith, R., \& Wahls, H. (1969). Consolidation under Constant Rates of Strain. Journal of the Soil Mechanics and Foundations Division, 95(SM2).

Tavenas, F., Blanchette, G., Leroueil, S., Roy, M., \& La Rochelle, P. (1975). Difficulties in the In Situ Determination of K0 in Sensitive Clays. Proceedings of the Specialty Conference on In Situ Measurements of Soil Properties (pp. 450-476). Raleigh, N.C.: American Society of Civil Engineers (ASCE).

Trautwein. (2001). Sigma-1 CRS - Instruction Manual - Version 1.01.01. Houston, TX: Trautwein Soil Testing Equipment Company.

Umar, M., \& Sadrekarimi, A. (2016). Accuracy of Determining Pre-Consolidation Pressure from Laboratory Tests. Canadian Geotechnical Society Conference. Vancouver.

Wang, L., \& Frost, J. (2004). Dissipated Strain Energy Method for Determining Preconsolidation Pressure. Canadian Geotechnical Journal, 41(4), 760-768.

Wissa, A., Christian, J., Davis, E., \& Heiberg, S. (1971). Consolidation Testing at Constant Rates of Strain. Journal of Soil Mechanics and Foundations Divisions, 97(10), 1393-1413.

Yoon, H.-K., Lee, C., Kim, H.-K., \& Lee, J.-S. (2011). Evaluation of Preconsolidation Stress by Shear Wave Velocity. Smart Structures and Systems, 7(4), 275-287. 